
MULTI-ITEM TWO-ECHELON SPARE PARTS INVENTORY CONTROL PROBLEM WITH BATCH ORDERING IN THE CENTRAL WAREHOUSE

A THESIS SUBMITTED TO

THE GRADUATE SCHOOL OF NATURAL AND APPLIED SCIENCES $\mathrm{OF}$ MIDDLE EAST TECHNICAL UNIVERSITY

$\mathrm{BY}$

ENGIN TOPAN

IN PARTIAL FULFILLMENT OF THE REQUIREMENTS FOR THE DEGREE OF DOCTOR OF PHILOSOPHY IN INDUSTRIAL ENGINEERING

OCTOBER 2010 
Approval of the thesis:

\section{MULTI-ITEM TWO-ECHELON SPARE PARTS INVENTORY CONTROL PROBLEM WITH BATCH ORDERING IN THE CENTRAL WAREHOUSE}

submitted by ENGIN TOPAN in partial fulfillment of the requirements for the degree of

Doctor of Philosophy in Industrial Engineering Department, Middle East Technical University by,

Prof. Dr. Canan Özgen

Dean, Graduate School of Natural and Applied Sciences

Prof. Dr. Sinan Kayalıgil

Head of Department, Industrial Engineering

Assist. Prof. Dr. Z. Pelin Bayındır

Supervisor, Industrial Engineering Dept., METU

Assist. Prof. Dr. Tarkan Tan

Co-supervisor, School of Industrial Engineering, TU Eindhoven

\section{Examining Committee Members:}

Prof. Dr. Ömer Kırca

Industrial Engineering Dept., METU

Assist. Prof. Dr. Z. Pelin Bayındır

Industrial Engineering Dept., METU

Prof. Dr. Nesim Erkip

Industrial Engineering Dept., Bilkent University

Prof. Dr. Geert-Jan Van Houtum

School of Industrial Engineering, TU Eindhoven

Assist. Prof. Dr. İsmail Serdar Bakal

Industrial Engineering Department, METU

Date: 
I hereby declare that all information in this document has been obtained and presented in accordance with academic rules and ethical conduct. I also declare that, as required by these rules and conduct, I have fully cited and referenced all material and results that are not original to this work.

Name, Last Name: ENGIN TOPAN

Signature 


\begin{abstract}
MULTI-ITEM TWO-ECHELON SPARE PARTS INVENTORY CONTROL PROBLEM WITH BATCH ORDERING IN THE CENTRAL WAREHOUSE

\author{
Topan, Engin \\ $\mathrm{Ph} . \mathrm{D}$, Department of Industrial Engineering \\ Supervisor : : Assist. Prof. Dr. Z. Pelin Bayındır \\ Co-Supervisor : Assist. Prof. Dr. Tarkan Tan
}

October 2010, 187 pages

In this dissertation, we consider a multi-item two-echelon inventory distribution system in which the central warehouse operates with $(Q, R)$ policy, and each local warehouse implements base-stock policy. The objective is to find the policy parameters minimizing the relevant system-wide costs subject to an aggregate mean response time constraint at each facility.

We first propose an exact solution procedure based on a branch-and-price algorithm to find the relevant policy parameters of the system considered. Then, we propose four alternative heuristics to find the optimal or near-optimal policy parameters of large practical-size systems. The first heuristic, which we call the Lagrangian heuristic, is based on the simultaneous approach and relies on the integration of a column generation method and a greedy algorithm. The other three heuristics are based on the sequential approach, in which first the order quantities are determined using a batch size heuristic, then the reorder levels at the central warehouse and the basestock levels at the local warehouses are determined through the same method used 
for the Lagrangian heuristic. We also propose a lower bound for the system-wide cost. Later, we extend our study to compound Poisson demand.

The performance of the Lagrangian heuristic is found to be extremely well and improves even further as the number of parts increases. Also the computational requirement of the heuristic is quite tolerable. This makes the heuristic very promising for large practical industry-size problems. The performance of the sequential heuristics is also satisfactory, but not as much as the Lagrangian heuristic.

Keywords: inventory, two-echelon, multi-item, batch ordering, spare parts 


\title{
Öz
}

\section{MERKEZİ DEPODA TOPLU SIPARISŞIN OLDUĞU ÇOK ÜRÜNLÜ İKİ SEVIYELI YEDEK PARÇA ENVANTER KƠNTROLÜ}

\author{
Topan, Engin \\ Doktora, Endüstri Mühendisliği Bölümü \\ Tez Yöneticisi : Yrd. Doç. Dr. Z. Pelin Bayındır \\ Ortak Tez Yöneticisi : Yrd. Doç. Dr. Tarkan Tan
}

Ekim 2010, 187 sayfa

$\mathrm{Bu}$ tezde, merkezi deponun $(\mathrm{Q}, \mathrm{R})$ politikası ile, ve yerel depoların ise seviye esaslı envanter sistemi ile çalıştığı çok ürünlü, iki seviyeli yedek parça envanter dağıtım sistemi incelenmiştir. Amaç depolardaki ortalama toplaşık yanıt zamanı kısıtları altında sistemin bütününe ait maliyetleri enazlayan politika parametrelerinin bulunmasıdır.

Uygun politika parametrelerini bulmak için ilk olarak kesin çözüm prosedürü olan dal-ve-fiyatlandırma algoritması tasarlanmıştır. Daha sonra, büyük ölçekli gerçek sistemlerin en iyi yada en iyiye yakın politika parametrelerini bulmak amacıyla dört adet alternatif sezgisel metot geliştirilmiştir. Lagranj sezgiseli adını verdiğimiz birinci sezgisel metot, eşzamanlı yaklaşım baz alınarak sütun türetimi ve obur algoritma metotlarının birleşimine dayanmaktadır. Diğer üç sezgisel metot ise, ilk olarak sipariş miktarının bölüt büyüklüğü sezgiseliyle, daha sonra merkezi deponun yeniden 1smarlama düzeyinin ve yerel depoların seviye esaslı envanter düzeylerinin Lagranj sezgiselinde kullanılan yöntemlerin kullanılmasıyla belirlendiği ardışık yaklaşıma dayanmaktadır. Daha sonra, geliştirdiğimiz yöntemler bileşik Poisson talep varsayımlı 
modeli kapsayacak şekilde genişletilmiştir.

Lagranj sezgiselinin performansı çok iyi olup, parça sayısı arttıkça sezgiselin performansının daha da iyileştiği görülmüştür. Ayrıca sezgiseli hesaplamak için gereken zaman kabul edilebilir ölçülerdedir. Bunlar, sezgisel metodun büyük ölçekli gerçek endüstriyel sistemlerde kullanılabilmesi için umut verici olduğunu göstermektedir. Lagranj sezgiseli kadar olmasa da politika parametrelerinin ardışık belirlenmesini de tatmin edici sonuçlar vermiştir.

Anahtar Kelimeler: envanter, iki seviyeli, çok ürünlü, toplu sipariş, yedek parça 
To my wife and my family,

and

in the memory of my grandfather Yusuf Engin 


\section{ACKNOWLEDGMENTS}

I would like to thank my thesis supervisor Assist. Prof. Dr. Z. Pelin Bayındır and cosupervisor Assist. Prof. Dr. Tarkan Tan for their supervision, guidance and support throughout the dissertation. I heartily consider myself very lucky to have worked with them.

I owe special thanks to the Thesis Monitoring Committee Members, Prof. Dr. Geert Jan Van Houtum, Assist. Prof. Dr. Seçil Savaşaneril Tüfekçi and Assist. Prof. Dr. İsmail Serdar Bakal for their monitoring and valuable contributions throughout my dissertation.

I also thank to Prof. Dr Ömer Kırca, Prof. Dr. Nesim Erkip for serving as members of my thesis defense jury and for their valuable contributions and comments.

I would like to express my sincere gratitude to my dean Prof. Dr. Levent Kandiller. His guidance makes it possible to take the correct steps before and during the dissertation.

I also thank everyone who directly or indirectly contributed to my study. Among those, I owe special thanks to my chairman Assist. Prof. Dr. Nureddin Kırkavak and Assist. Prof. Dr. Haluk Aygüneş for their understanding and support that I need especially towards the end of my dissertation.

Special thanks go to my love, İpek, who has given me strength and morale that I need during the dissertation. She has provided me everything that one needs during a dissertation. I owe my deepest thanks to my parents, Gülsiye and Hüseyin Topan for all the sacrifices they made. I also thank my brother Ersin Topan, and my parents-inlaw İnci and Oğuz Seyran for their support during my study.

I would like to mention that my dissertation is funded by TÜBİTAK BIDDEB-2211. Therefore, I would also like to thank to TÜBITAK for their financial support during the dissertation. 


\section{TABLE OF CONTENTS}

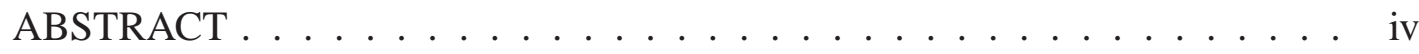

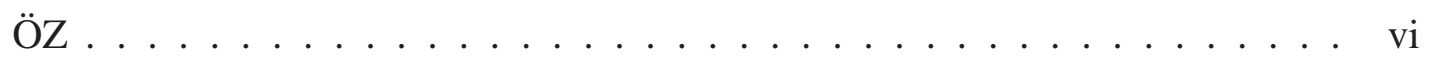

ACKNOWLEDGMENTS . .................... ix

TABLE OF CONTENTS . . . . . . . . . . . . . . . . . .

LIST OF TABLES . . . . . . . . . . . . . . . . . xiii

LIST OF FIGURES . . . . . . . . . . . . . . . . . . . . xvi

CHAPTERS

1 INTRODUCTION $\ldots \ldots \ldots \ldots$

2 LITERATURE REVIEW . . . . . . . . . . . . . . . . . . . 12

2.1 Multi-Echelon Inventory Distribution Systems . . . . . . . 12

2.1.1 Single-Item Systems _ . . . . . . . . . . 18

2.1.2 Multi-Item Systems . . . . . . . . . . . 33

$2.2 \quad$ Sequential Heuristics _. . . . . . . . . . . . . 39

3 AN EXACT SOLUTION PROCEDURE . . . . . . . . . . . . . . 42

$3.1 \quad$ The model . . . . . . . . . . . . . . . 43

3.2 The branch-and-price algorithm . . . . . . . . . . . . 49

3.2.1 Obtaining the Lagrangian dual bound for the problem: Column Generation Method . . . . . . . 50

3.2.2 Solution Procedure for Subproblems: Single-item Two-echelon Batch Ordering Problem . . . . . . . 54

3.2.2.1 Finding Optimal Solution for Subproblems for Given Values of Reorder Level and Order Quantity . . . . . . . . . 59

3.2.3 Generating Upper Bounds: Greedy Algorithm . . . 61 
$3.3 \quad$ Computational Results . . . . . . . . . . . . . . . 62

$3.4 \quad$ Conclusion . . . . . . . . . . . . . 62

4 HEURISTIC PROCEDURES . . . . . . . . . . . . . . . . . . 65

$4.1 \quad$ Lagrangian Heuristic _. . . . . . . . . . . . 66

4.2 Sequential Heuristics . . . . . . . . . . . . . 66

$4.3 \quad$ Asymptotic analysis of the Lagrangian dual bound . . . . . . 70

$4.4 \quad$ Computational Study _. . . . . . . . . . . . 76

4.4.1 Experimental Design . . . . . . . . . . 77

4.4.2 Performance of the Lagrangian Dual Bound . . . . 80

4.4.3 Performance of the Lagrangian Heuristic . . . . 81

4.4.4 Performance of the Sequential Heuristics . . . . 90

4.4.5 Computational requirements of the solution procedures and experiments with Practical-Size Problems 94

$4.5 \quad$ Conclusion . . . . . . . . . . . . . . . 100

5 EXTENSIONS TO COMPOUND POISSON DEMAND . . . . . . 103

5.1 The model . . . . . . . . . . . . . . . . . . . 104

5.1.1 Obtaining the Lead Time Demand Distributions . . 108

5.2 An Exact Evaluation Based on a Flow-Unit Method .... . . 109

5.3 Approximate Evaluation Methods Based on a Disaggregation Method ... . . . . . . . . . . . . . 111

$5.4 \quad$ Solution Procedures . . . . . . . . . . . . . . . . . 114

$5.5 \quad$ Computational Study _. . . . . . . . . . . . 115

5.5.1 Experimental Design . . . . . . . . 118

5.5.2 Performance of the Lagrangian Heuristic under the exact evaluation . . . . . . . . . . . . . . 122

5.5.3 Performance of the heuristics under the approximate evaluation methods . . . . . . . . . . . 127

5.5.3.1 The performance of the Lagrangian heuristic under the approximate evaluation methods . . . . . . . . 127

5.5.3.2 Performance of the sequential heuristics under the approximate evaluation methods . . . . . . . . . 140 
5.5.4 Performance of the Lagrangian dual solution under the approximate evaluation methods . . . . . . 142

5.5.5 Performance of the heuristics under the approximate evaluation methods in large-scale problems . 145

$5.6 \quad$ Conclusion . . . . . . . . . . . . . . . . 154

CONCLUSIONS AND FUTURE DIRECTIONS . . . . . . . . . 156

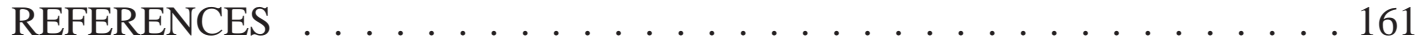

APPENDICES

A EXTENDING THE SOLUTION PROCEDURE FOR SUBPROBLEMS TO COMPOUND POISSON DEMAND SETTING . . . . . . 166

A.1 Solution Procedure for Subproblems: Single-item Two-echelon Batch Ordering Problem . . . . . . . . . . . . . 166

A.1.1 Finding Optimal Solution for Subproblems for Given Values of Reorder Level and Order Quantity . . . . 169

B THE RESULTS OF THE EXPERIMENTERS FOR TESTBED 1 FOR THE SEQUENTIAL HEURISTICS . . . . . . . . . . . . . 171

C NOTATIONS USED IN THE DISSERTATION . . . . . . . . . . 181

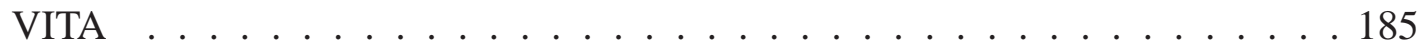




\section{LIST OF TABLES}

\section{TABLES}

Table 2.1 Taxonomy of Related Literature on Multi-Echelon Inventory Systems. 15

Table 3.1 General Notation. . . . . . . . . . . . . . . . . . . . . 45

Table 3.2 Parameter values for the testbed. . . . . . . . . . . . . 63

Table 3.3 CPU times for different problem sizes. . . . . . . . . . . . 63

Table 4.1 Heuristics proposed. . . . . . . . . . . . . . . . . . . . 70

Table 4.2 Base case setting for the experiments. . . . . . . . . . . . . . . 79

Table 4.3 Multipliers for the average demand rates, average unit variable cost of parts, average fixed ordering costs and target aggregate mean response times.. . . . . . . . . . . . . . . . . 79

Table 4.4 Effect of the parameters on the performance of the Lagrangian dual bound. . . . . . . . . . . . . . . . . . 82

Table 4.5 Effect of the parameters on the performance of the Lagrangian heuris-

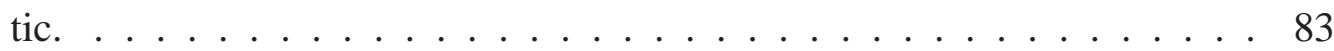

Table 4.6 Effect of the parameters on the performance of the sequential heuris-

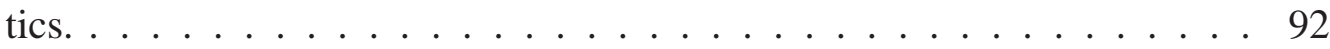

Table 5.1 General Notation. . . . . . . . . . . . . . . . . . . . . 107

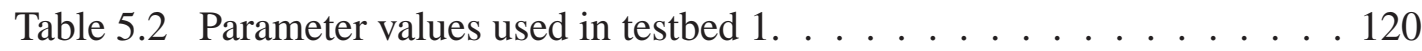

Table 5.3 Parameter values used in testbed 2. . . . . . . . . . . . . . . 121

Table 5.4 Parameter values used in testbed 3 . . . . . . . . . . . . . . . . 122

Table 5.5 Effects of parameters on the performance of the Lagrangian heuristic when the exact evaluation method is used. . . . . . . . . . . . . . . 124 
Table 5.6 Computational requirements of the Lagrangian heuristics under the exact and approximate evaluation methods (CPU time in sec.) . . . . . . 127

Table 5.7 Effects of parameters on the performance of the Lagrangian heuristic under the approximate evaluation methods $\left(P C D_{L H}\right) \ldots \ldots 132$

Table 5.8 Effects of parameters on the performance of the Lagrangian heuristic under the approximate evaluation methods (Number/percentage of feasible solutions). . . . . . . . . . . . . . . . . . . . . . . . 134

Table 5.9 Effects of parameters on the performance of the Lagrangian heuristic under the approximate evaluation methods $(R D F R)$. . . . . . . 136

Table 5.10 Computational requirements of the sequential heuristics under the approximate evaluation methods (CPU time in sec) . . . . . . . . . 143

Table 5.11 Effects of parameters on the performance of the Lagrangian dual solution under the approximate evaluation methods $(P G A P)$. . . . . . . 144

Table B.1 Effects of parameters on the performance of the sequential heuristics $S_{1}$ under the approximate evaluation methods $\left(P C D_{L H}\right) \ldots \ldots 172$

Table B.2 Effects of parameters on the performance of the sequential heuristics $S_{1}$ under the approximate evaluation methods (Number/percentage of feasible solutions). . . . . . . . . . . . . . . . . 173

Table B.3 Effects of parameters on the performance of the sequential heuristics $S_{1}$ under the approximate evaluation methods $(R D F R) . \ldots 174$

Table B.4 Effects of parameters on the performance of the sequential heuristics $S_{2}$ under the approximate evaluation methods $\left(P C D_{L H}\right) \ldots \ldots 175$

Table B.5 Effects of parameters on the performance of the sequential heuristics $S_{2}$ under the approximate evaluation methods (Number/percentage of feasible solutions). . . . . . . . . . . . . . . . . 176

Table B.6 Effects of parameters on the performance of the sequential heuristics $S_{2}$ under the approximate evaluation methods $(R D F R) . \ldots 177$

Table B.7 Effects of parameters on the performance of the sequential heuristics $S_{3}$ under the approximate evaluation methods $\left(P C D_{L H}\right) \ldots \ldots . \ldots 178$ 
Table B.8 Effects of parameters on the performance of the sequential heuristics $S_{3}$ under the approximate evaluation methods (Number/percentage of feasible solutions). . . . . . . . . . . . . . . . . . . . . . 179

Table B.9 Effects of parameters on the performance of the sequential heuristics $S_{3}$ under the approximate evaluation methods $(R D F R) \ldots \ldots \ldots$ 


\section{LIST OF FIGURES}

\section{FIGURES}

Figure 3.1 The Flowchart of the Branch-and-Price Algorithm. . . . . . . . . . 51

Figure 4.1 The Flowchart of the Lagrangian Heuristic. . . . . . . . . . . . . 67

Figure 4.2 The Flowchart of the Sequential Heuristics. . . . . . . . . . . . . 69

Figure 4.3 Effect of number of parts on the performance of the Lagrangian dual bound. . . . . . . . . . . . . . . . . . . . 84

Figure 4.4 Sensitivity Analysis: The effects of parameters on the performance of the Lagrangian heuristic - average demand rates, average unit variable cost of parts, average fixed ordering costs and target aggregate mean response times. . . . . . . . . . . . . . . . . . . . . . 86

Figure 4.5 Sensitivity Analysis: The effects of parameters on the performance of the Lagrangian heuristic - number of parts, number of local warehouses. 88

Figure 4.6 Performance of the heuristics with respect to number of parts and number of local warehouses $-P C D \ldots \ldots . \ldots 95$

Figure 4.7 Performance of the heuristics with respect to number of parts and number of local warehouses - ACD . . . . . . . . . . . . . . . 97

Figure 4.8 Computational requirements of the heuristics with respect to number of parts and number of local warehouses. . . . . . . . . . . . . . . . 99

Figure 5.1 Effect of variance-to-mean ratio on the lead time demand distribution, its mean and variance. . . . . . . . . . . . . . . . . . 123

Figure 5.2 Effect of variance-to-mean ratio on the lead time demand distribution, its mean and variance. . . . . . . . . . . . . . . . 126

Figure 5.3 Effect of the number of parts on the performance of the Lagrangian heuristic under various approximations $\left(P C D_{L H}\right) . \ldots . \ldots 129$ 
Figure 5.4 Effect of the number of parts on the performance of the Lagrangian heuristic under various approximations $(R D F R)$. . . . . . . . . . . 137

Figure 5.5 Effect of the number of parts on the performance of the Lagrangian dual solution under various approximations $\left(P G A P^{\prime}-\right.$ Number of local

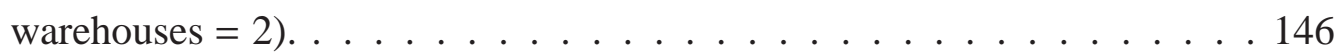

Figure 5.6 Effect of the number of parts on the performance of the Lagrangian dual solution under various approximations $\left(P G A P^{\prime}-\right.$ Number of local

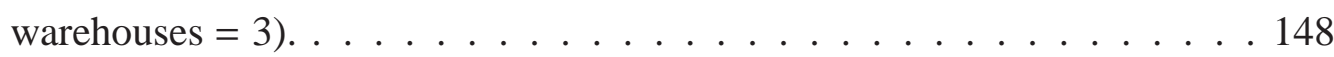

Figure 5.7 Effect of the number of parts on the performance of the Lagrangian and the sequential heuristic under the two-moment approximation for outstanding orders $\left(P C D_{\text {LHapp }}\right) \ldots \ldots \ldots$. . . . . . . . . . . 150

Figure 5.8 Effect of the number of parts on the performance of the Lagrangian heuristic under various approximations $\left(P C D_{\text {LHapp }}\right) \ldots \ldots \ldots 1$

Figure 5.9 Computational requirements of the heuristics evaluated under the two-moment approximation for outstanding orders (CPU time in sec). . . . 152

Figure 5.10 Computational requirements of the Lagrangian heuristic under various approximations $($ CPU time in sec) $\ldots \ldots \ldots 153$

Figure 5.11 Average stocking level obtained by the Lagrangian heuristic evaluated under the two-moment approximation for outstanding orders. . . . . 154 


\section{CHAPTER 1}

\section{INTRODUCTION}

Today's manufacturing companies differ from the traditional ones mainly in the sense that they do not only manufacture products, but also offer after-sales services for their customer. Deloitte (2006) investigates the largest manufacturing companies in the world, and reports that these manufacturing companies make $46 \%$ of the profits from their service and parts operations. Similarly, AMR Research (2002), a research company focusing on the global supply chain issues, reports that manufacturing companies make nearly $25 \%$ of their revenues and 40 to $50 \%$ of their profits from after-sales services. All these reports indicate that after-sales services are highly important as well as attractive for the manufacturers.

The after-sales services that a typical manufacturer offers may involve any kind of service activities such as provisioning of spare parts, preventive and/or corrective maintenance, repair activities. Spare parts management is in the center of all these service activities.

A spare part is often a component of a larger system that has a critical function such a machine, an equipment or a vehicle. For the customers of manufacturers, equipment breakdowns are of essential importance since these may lead to discontinuing a critical process at the customer site. This often results in high down-time costs. Therefore, availability of the parts becomes critically important for the customers. In such an environment, customers are protected against down-time risks by service level agreements (SLAs). In these agreements, the manufacturer promises service requirements of customers, which are usually expressed as a target level on a certain service measure, such as fill rate, probability of no-stockout and response time. 
However, the demand for spare parts is highly unpredictable. It is also difficult to control the demand, although there are some tools for that purpose, e.g., applying forecasting methods, scheduled preventive maintenance, advertising and organizing sales campaigns. Consequently, the significance of availability and the unpredictable nature of the demand put pressure on the manufacturers to hold inventory. Nevertheless, running a spare parts inventory system costs to the manufacturer. The basic trade off between costs and service requirements in spare parts logistics makes spare parts inventory control one of the most critical after-sales services for the manufacturers.

This dissertation is motivated by a spare parts inventory control problem that we observe in two different capital goods manufacturers providing equipments and services for capital intensive markets. Although the problem is based on these two manufacturers, it generalizes to other manufacturers facing a similar problem.

The first manufacturer that we consider is a leading supplier of advanced tools to the nanotechnology market. The manufacturer, as a service provider, operates a spare parts inventory system consisting of a central warehouse and a number of local warehouses at different locations to be responsive to their customers geographically dispersed around the world and manages nearly 14,000 parts involving very cheap parts as well as highly expensive ones, e.g., the value of the most expensive part exceeds hundreds of thousands of euros. The value of the spare parts only in the central warehouse accounts for more than 12 million dollars. The other manufacturer is a leading manufacturer of industrial printing systems. They operate a similar inventory system, involving more (approximately 20,000), but relatively less expensive parts compared to the first manufacturer. The value of the spare parts only in the central warehouse accounts for almost 2 million euros. Although the manufacturers serve totally different markets with different part characteristics, the following observations are common for them.

- Since the manufacturers should supply spare parts for different customers at different locations, they operate an inventory distribution system that consists of a number of local warehouses at different locations and a central warehouse replenishing them. This type of two-echelon distribution systems are prevalent in spare parts logistics (Cohen et al. 1997). 
- All warehouses can respond to customer requirements. Hence, the central warehouse has both internal requests from the local warehouses and external requests from the customers.

- Most of the spare parts that the manufacturers provide are rarely used, slow moving items, a majority of which have a demand rate of less than 5 parts per year. In addition, since ordering between the lower and the upper echelons is internal and automated, fixed ordering costs are insignificant at the lower echelon facilities. Hence, the batch sizes are low, often equivalent to one. Therefore, both manufacturers operate under a continuous-review installation-stock basestock policy, i.e., $(S-1, S)$ policy, at the lower echelon facilities. This situation is common and often justified in other spare parts inventory control practices (Wong et al. 2007b, Hopp et al. 1999).

- At the central warehouse, parts move faster due to the accumulation of internal demands from local warehouses. Moreover, the central warehouse typically replenishes from external suppliers, resulting in high fixed procurement/ transportation costs. Therefore, the manufacturers place orders in batches instead of individual units at the upper echelon, i.e., they apply a batch ordering policy at the central warehouse. There are situations where batching decisions are motivated by aggregate performance targets on the order frequencies at the central warehouse or production smoothing requirements of a third-party supplier (Hopp et al. 1999, Al-Rifai and Rossetti 2007), although this is not the case for the manufacturers considered. Under these conditions, it is more reasonable for the central warehouse to operate under a continuous-review installation-stock reorder point, order quantity policy, i.e., $(Q, R)$ policy. In the manufacturers that we consider, the corresponding policy parameters are determined based on the experience of the inventory controller or some simple heuristics.

- Each of these manufacturers produces equipment that has a critical function for their customers, e.g., printing machine, electron microscope etc. Therefore, for the customers of these manufacturers, resulting down-time costs are expressed on the order of thousands of euros per hour. For both manufacturers, service levels committed to customers are formally defined via SLAs. The first manufacturer sets the response time, time to respond for a customer request as 
a service measure. The second manufacturer sets fill rate as the performance measure. Although the manufacturers differ in the service level types, the SLAs play an important role in their spare parts inventory system.

- SLAs that are defined between the manufacturers and the customers can typically be classified into two groups: Under the "item approach", a target service level is defined for each individual part. It is widely considered in the inventory literature (Thonemann et al. 2002). Another approach is the "system approach", in which a target service level is defined for the demand weighted average of the relevant performance measure over all parts. Hence, the system approach defines an aggregate service measure. Although the number of end products that a typical manufacturer produces is quite limited, the number of spare parts associated with the products can be very large, often on the order of thousands or ten thousands. Since customers are primarily interested in their equipment or entire system being up and running, setting a target service level for each part does not make sense for them. Instead, they are interested in the availability of parts at an aggregate level. Since the system approach is based on the demand weighted average of the relevant performance over all parts, it enables holding more inventories for cheap parts while fewer for expensive parts. This brings substantial savings in inventory holding costs in comparison with the situation under the item approach (Thonemann et al. 2002) to the manufacturer. Hence, the system approach is more applicable and widely adopted in the SLAs for spare parts (Hopp et al. 1999, Al-Rifai and Rossetti 2007, Çağlar et al. 2004, Wong et al. 2007b), which is also the case for the manufacturers that we consider.

In this dissertation, similar to the two examples that we mention, we consider a multiitem two-echelon spare-parts inventory distribution system consisting of a number of local warehouses and a central warehouse. Local warehouses operate under an a continuous-review installation-stock $(S-1, S)$ base-stock policy, while the central warehouse operates under a continuous-review installation-stock $(Q, R)$ policy. Our focus is on the corrective maintenance activities of the service provider. Also, our emphasize is on the consumable parts, that is, we ignore the repairable parts or we model them as if they are consumable. For this system, our objective is to find the 
optimal or the near-optimal policy parameters minimizing the expected system-wide inventory holding and fixed ordering costs subject to an aggregate mean response time constraint.

Finding the optimal or near-optimal policy parameters of such an inventory system is generally difficult. The main reasons are as follows:

- Even a medium scale inventory system involves thousands of stock keeping units, for each of which the policy parameters should be optimized. Furthermore, under a system approach, the policy parameters for each part interacts with the others through constraints on an aggregate performance measure. This makes the resulting optimization problem very complex.

- The evaluation of the objective function and the constraints of such an optimization problem requires evaluating the probability distributions of the inventory levels, which are difficult to compute even in a single-item case.

- Finding the optimal policy parameters of a typical system under batch ordering is much more involved compared to the one in which each facility operates under a pure base-stock policy since the reorder levels and the order quantities at the central warehouse need to be determined simultaneously with the basestock levels at each local warehouse for each part, where the policy parameters of the parts interact with each other.

Since finding the policy parameters of multi-item two-echelon inventory distribution systems is difficult, all the solution procedures developed for these systems rely on heuristics. To the best of our knowledge, there is no exact solution algorithm developed for multi-item two-echelon inventory distribution systems. Even though approximations and heuristics are prevalent approaches, an exact solution procedure can be considered for two reasons:

- Cost reductions: The average inventory value of a typical company in the capital goods industry is of the order of tens of millions of euros (Cohen 1997, Sleptchenko 2002). This means that even a small percentage reduction in inventories may correspond to savings on the order of hundreds of thousands of euros. 
- Benchmarking purposes: Due to the lack of an exact solution algorithm, heuristics in the literature are usually compared to each other, or lower bounds, or simulation-based optimization results. However, not all of these benchmark solutions can guarantee satisfactory performance: Their performance may differ depending on the problem parameters leading to difficulties in assessing the performance of a proposed heuristic (Al-Rifai and Rossetti 2007, Çağlar et al. 2004). In addition, it is possible to use an exact solution algorithm to test the performance of a lower bound for small size problems, before using it as a benchmark for larger problems.

In this dissertation, we aim to propose an exact solution procedure to find the optimal policy parameters of the multi-item two-echelon inventory distribution system considered. Nevertheless, for practical size problems, an exact solution requires significant computational effort. However, considering that even a moderate industry-size problem requires to optimize the policy parameters of thousands of items, an efficient solution method is of critical importance. Hence, one may need to apply heuristic approaches for the solution of practical size problems. There are many efforts devoted to propose heuristics to find the policy parameters of multi-item multi-echelon systems under pure base-stock policy (Çağlar et al. 2004, Wong et al. 2007b, Caggiano et al. 2007) and batch ordering policy (Hopp et al. 1999, Al-Rifai and Rossetti 2007). Almost all of these heuristics are based on approximate evaluation of the probability distributions of the inventory levels, hence, they do not guarantee feasible solutions with respect to constraints on service levels. The only heuristic that is based on an exact evaluation method is proposed by Wong et al. (2007b), which is developed for systems under base-stock policy. Also, for many of these heuristics, finding the policy parameters of a practical size problem becomes an issue. In this dissertation, one of our aim is to develop heuristics guaranteeing feasibility and at the same time yielding satisfactory results in terms of both the relative errors and the computation time for practical-size systems.

As an alternative to an exact solution procedure, one may consider a heuristic approach to find the policy parameters of the inventory system that we pose. A common practice is to follow a sequential approach, which assumes the dominance of the batching decisions over the others, and hence it necessitates determining the batch 
sizes first (in most applications independent of the service level requirements), and then the other policy parameters. The method brings a significant computational saving and also results in very low percentage cost penalty in single-item single-echelon systems which is verified both empirically and theoretically by several researches (Zheng 1992, Axsäter 1996, Silver et al. 1998, Gallego 1998). Due to its excellent performance in single-item single-echelon systems, it is also widely used in general system settings (Hopp et al. 1997, Axsäter 1998, Hopp et al. 1999, Axsäter 2003) as well as in practical applications, e.g., the manufacturers considered in our dissertation adopt the sequential approach to find the policy parameters of their inventory control systems. Although the sequential approach is widely used in multi-item multiechelon inventory control applications, its performance has not been fully assessed in the literature. One of the objectives of this dissertation is to investigate the performance of the sequential approach in a multi-item two-echelon inventory control system.

Just like finding the optimal policy parameters, finding an efficient and tractable benchmark solution, e.g., a tight lower bound on the optimal expected total cost, for an inventory control policy for multi-item multi-echelon system is also difficult. This makes it hard to evaluate the performance of the heuristics (Çağlar et al. 2004, Al-Rifai and Rossetti 2007). In this dissertation, we also propose a lower bound that can be used as a benchmark solution to test the performance of the heuristics.

Since most of the spare parts are slow moving items and their demand is intermittent, the Poisson distribution often provides a reasonable representation of the demand process in many situations. Hence, it is quite common in the literature to assume that demand is Poisson. However, the Poisson distribution is not verified for parts whose demand sizes varies from one transaction to another and also those parts whose total demand variance is higher. For instance in the case of preventive maintenance, the number of spare parts demanded is quite often more than one. In this situation, the Poisson demand assumption, which relies on the unit size demand assumption, fails. As opposed to Poisson distribution, the compound Poisson distribution, where customer arrivals occur according to a Poisson process with random demand sizes, provides a better representation of the demand process for certain spare parts. There are empirical results verifying that compound Poisson distribution better characterizes 
the demand distribution for spare parts (Eaves 2002). In the two manufacturing firms that we observe, there are spare parts for which a customer's demand is more than one. While proposing solution procedures for the problem that we introduce, we also consider these issues and develop solution procedures for both Poisson and compound Poisson settings.

Our work contributes to the relevant literature in the following directions:

- In Chapter 3, we propose an exact solution procedure to find the optimal policy parameters minimizing the system-wide expected inventory holding and fixed ordering cost subject to an aggregate mean response time constraint at each facility. We experimentally show that the branch-and-price algorithm can be used in applications as long as the number of items and the number of warehouses are limited. Even if this is the case, an exact solution is desirable due to significant cost reductions and benchmark purposes. To the best of our knowledge, our work is the first to propose an exact solution procedure to find the optimal policy parameters of a multi-item two-echelon inventory system. A slightly different version of this chapter is published in Operations Research Letters (Topan et al. 2010a).

- In Chapter 4, we propose four alternative heuristics to find the policy parameters of large, practical-size multi-item two-echelon inventory control systems with batch ordering at the central warehouse. Our heuristics are based on the exact evaluation of the probability distributions of the inventory levels. Hence, in contrast to most of the studies in the literature, our heuristics guarantee feasible solutions. The first heuristic, which we call the Lagrangian heuristic, is based on the simultaneous approach and relies on the integration of a column generation method and a greedy algorithm. The other three heuristics are based on the sequential approach, in which first the order quantities are determined using a batch size heuristic, then the reorder levels at the central warehouse and the base-stock levels at the local warehouses are determined through the same method used for the Lagrangian heuristic, i.e., a column generation and a greedy algorithm. These three heuristics differ in the batch size heuristic used. We have a working paper covering the issues in Chapter 4 (see Topan et al. 
2010b).

- In Chapter 3, we also propose a lower bound for the optimal expected total cost. Later, in Chapter 4 we show that this bound is asymptotically tight in the number of parts. Considering the difficulties encountered in evaluating the performance of heuristics for different multi-item two-echelon inventory systems in the literature (Çağlar et al. 2004, Al-Rifai and Rossetti 2007), the lower bound that we propose also makes a significant contribution to the relevant literature.

- All our developments and findings presented in Chapters 3 and 4 are for a pure Poisson demand model. Later, in Chapter 5 by using our findings as building blocks, we extend the solution procedures for compound Poisson demand model. To the best of our knowledge, our work is the first to propose heuristics for finding the parameters of a multi-item two-echelon inventory control systems facing a compound Poisson process. Since the exact evaluation of this system is intractable, for large practical size problems, we consider four approximate evaluation methods. The first approximation relies on a disaggregation method, which is exact for pure Poisson demand. The other two are based on two-moment approximations, one of which is an extension of an existing method, while the other is considered for the first time in this work. The forth one is the extension of the Multi-Echelon Technique for Recoverable Item Control (METRIC) to our problem. We also compare the performances of these approximations. To the best of our knowledge, there is no previous work that compares the approximations commonly used in the literature under compound Poisson demand model. We also have a working paper covering the issues in Chapter 5 (see Topan et al. 2010c).

Our findings can be summarized as follows:

- In Chapter 4, we empirically show that the performance of the Lagrangian heuristic is quite well. As the number of parts increases, the performance of the heuristic improves further, making the heuristic very promising for practical applications. The computational requirement of the heuristic is quite tolerable. 
To be more specific, the experiment with 10,000 parts and 12 warehouses reveals that the relative difference between the expected total cost of the solution obtained by the Lagrangian heuristic and the lower bound is $0.04 \%$; problems of this size can be solved within 12 hours on an Intel $3 \mathrm{GHz}$ processor with 3.5 GB RAM. That is, we propose an efficient and effective heuristic for large practical industry-size problems.

- In Chapter 4, we also show that some of the qualitative conclusions regarding the performance of the sequential approach in the single-item single-echelon literature (Zheng 1992, Axsäter 1996, Silver et al. 1998, Gallego 1998) do not hold for the multi-item two-echelon setting, which is more representative of practical situations. First, we empirically observe that the relative cost difference may reach up to $31.03 \%$, which is fairly high compared to findings in the aforementioned papers on single-item single-echelon systems. Second, the computation times required for sequential heuristics are comparable to that of the Lagrangian heuristic, showing that the computational advantages of the sequential determination of policy parameters are limited in multi-item systems.

- For the compound Poisson demand setting, the Lagrangian heuristic, which is quite efficient in terms of the computational effort for Poisson demand model is found to be tractable only for relatively small problems, e.g., problems with 100 parts and 3 local warehouses. This also shows that exact evaluation of the system is tractable for the compound Poisson demand only when the number of parts and the number of local warehouses are limited. We show that the Lagrangian heuristic obtained by using two-moment approximations yield substantially better results than the ones that are obtained by using the METRIC or the disaggregation approximation. Both two-moment approximations perform quite well. We also show that many of the results that are valid in the Poisson demand setting are also valid in the compound Poisson demand setting, e.g., the performance of the heuristics improves with the number of parts, the Lagrangian heuristic is superior to sequential heuristic as in the pure Poisson demand case.

The organization of this dissertation is as follows. Chapter 2 provides a review of the 
literature relevant to the dissertation. First, we review the literature on multi-echelon inventory distribution systems in Section 2.1. This covers the papers on single-item as well as multi-item systems, which are presented in Sections 2.1.1 and 2.1.2, respectively. Then, the papers related to sequential heuristics are provided in Section 2.2 .

In Chapter 3, we propose an exact solution procedure for the problem. First, in Section 3.1, we specify the problem environment and then formulate the problem. In Section 3.2, the branch-and-price algorithm and the basic procedures used in the algorithm are presented, such as column generation algorithm (Section 3.2.1), an algorithm used to solve the single-item problem (Section 3.2.2), a greedy algorithm used to obtain an upper bound for the branch-and-price algorithm (Section 3.2.3). Finally, in Section 3.4, we provide the computational results.

In Chapter 4, we develop the heuristics that we consider in the dissertation. Section 4.1 introduces the Lagrangian heuristic whereas Section 4.2 introduces the sequential heuristics. In Section 4.3, we study the asymptotic behavior of the lower bound. We also present theoretical results associated with the asymptotic performance. In Section 4.4, we report and discuss our computational results. Here, the performances of the Lagrangian dual bound (Section 4.4.2), Lagrangian heuristic (Section 4.4.3), the sequential heuristics (Section 4.4.4) as well as the computational requirements of the methods (Section 4.4.5) are discussed. Finally in Section 4.5, we draw the conclusions.

In Chapter 5, we extend the developments in this dissertation to compound Poisson demand setting. In Section 5.1, we present the compound Poisson demand model and then develop the exact (Section 5.2) and the approximate evaluation methods (Section 5.3) considered for this model. In Section 5.4, we describe how the heuristics are extended to compound Poisson demand. In Section 5.5, we report and discuss our computational results. Finally, we draw the conclusions and discuss possible extensions in Chapter 6. 


\section{CHAPTER 2}

\section{LITERATURE REVIEW}

This dissertation mainly contributes to the vast literature on multi-echelon inventory control systems. We present the papers related to this field in Section 2.1. Another direction of research related to our dissertation is the development of the sequential heuristics and investigation of their performance in single and multi-echelon inventory systems. The papers related to this field are reviewed in Section 2.2.

\subsection{Multi-Echelon Inventory Distribution Systems}

In our work, we consider a two-echelon inventory distribution system under continuous review installation-stock policies. Since the analysis of other types of systems, e.g., serial, assembly or more general systems; periodic review inventory systems; echelon-stock policies are quite different than the analysis of distribution systems under continuous review installation-stock policies, they are not included in this review. For studies on periodic review inventory systems and echelon-stock policies, the reader may refer to Cachon (2001); Chen and Zheng (1997), Axsäter (1997), Simchi-Levi and Zhao (2010) and the references there in, respectively. On the other hand, our review includes the papers on systems with repairable and condemned parts, even though we limit ourselves to the systems with consumable parts in this dissertation. Since the analysis of systems with repairable and condemned parts are not completely different than that of the consumable parts.

There are three main directions of research in multi-echelon inventory control: optimal policy characterization, policy evaluation and policy optimization (Simchi-Levi 
and Zhao 2010). The main focus of the optimal policy characterization is the determination of the optimal policy and identification of its certain characteristics. Among the papers that we review, none of the papers deal with this issue. Since determining the optimal policy for complex network structures like distribution systems is generally difficult, for these systems, a common approach is to adopt an appropriative inventory control policy (probably a suboptimal one) and then to find the parameters minimizing the system-wide cost for the given policy.

There are two main issues in this approach: (i) Policy evaluation, that is, derivation of the system-wide performance measures: In general, it is difficult to derive the steady-state probabilities of inventory and backorder levels in multi-echelon inventory distribution systems. The first stream of papers that we review is on policy evaluation. Most of these evaluation methods rely on approximations. Nevertheless, there are exact evaluation methods proposed as well. (ii) Policy optimization, that is, the development of methods to search for the optimal or near-optimal policy parameters: A second -but more recent- stream of papers that we review deals with the policy optimization of multi-echelon inventory systems by proposing either exact solution methods to guarantee optimality or heuristics to find optimal or near-optimal solutions or both.

Although there are many papers that focus on both policy evaluation and policy optimization in this review, they can be basically classified in one of these two main categories. Our work belongs to the policy optimization part of the literature. Although we resort to both exact and approximate evaluation methods to obtain the steady-state analysis of the system, the policy evaluation is not the main focus of our work. In this dissertation, for the Poisson demand case, we propose an exact as well as alternative heuristic procedures to find the policy parameters of our system. For the compound Poisson demand, we only consider heuristics. From the perspective of evaluation method, we consider an exact evaluation method for the Poisson demand setting, whereas for the analysis of the compound Poisson model, we consider both exact and the approximate evaluation methods.

The papers in the literature can also be classified with respect to the following characteristics in order to position our work in Chapters 3, 4 and 5. 
- Number of items: There are two main categories: single-item and multi-item. Many of the seminal works study single-item systems. More recent body of literature deals with multi-item systems. The main focus of latter group is the development of efficient and effective heuristics to obtain the optimal or nearoptimal policy parameters of industry-size systems, just like the one in this dissertation. Although multi-indenture models with single type of products can be considered as a multi-item system, their analysis differ from both those of single-item and multi-item systems. Hence, it is also possible to consider them as a third group.

- Number of echelons: The papers that we review either consider two-echelon or more than two echelon systems.

- Demand distribution: Most papers assume a Poisson demand model for the demand process. There are also papers considering compound Poisson model. There are only two papers considering a general demand distribution.

- Inventory policy: There are mainly two groups of inventory control policies considered in the papers. These are base-stock policies and batch ordering policies. For the pure Poisson case, $(S-1, S)$ base-stock and $(Q, R)$ policies are implemented. For the compound Poisson demand model, order-up-to $\mathrm{S}$ and $(n Q, R)$ policies are considered, which are the adapted versions of $(S-1, S)$ and $(Q, R)$ policies to compound Poisson demand.

- Service motivator: There are two streams of papers according to the service motivator in the papers. The first stream of papers considers a cost model, whereas the second stream considers a service-constrained models.

A complete summary of the papers that we review and their position in the literature based on our taxonomy is given in Table 2.1. The details of these papers are discussed in the following section. In the following sections, we first review the papers on single-item systems, then we review the papers most relevant to this dissertation, multi-item systems. 


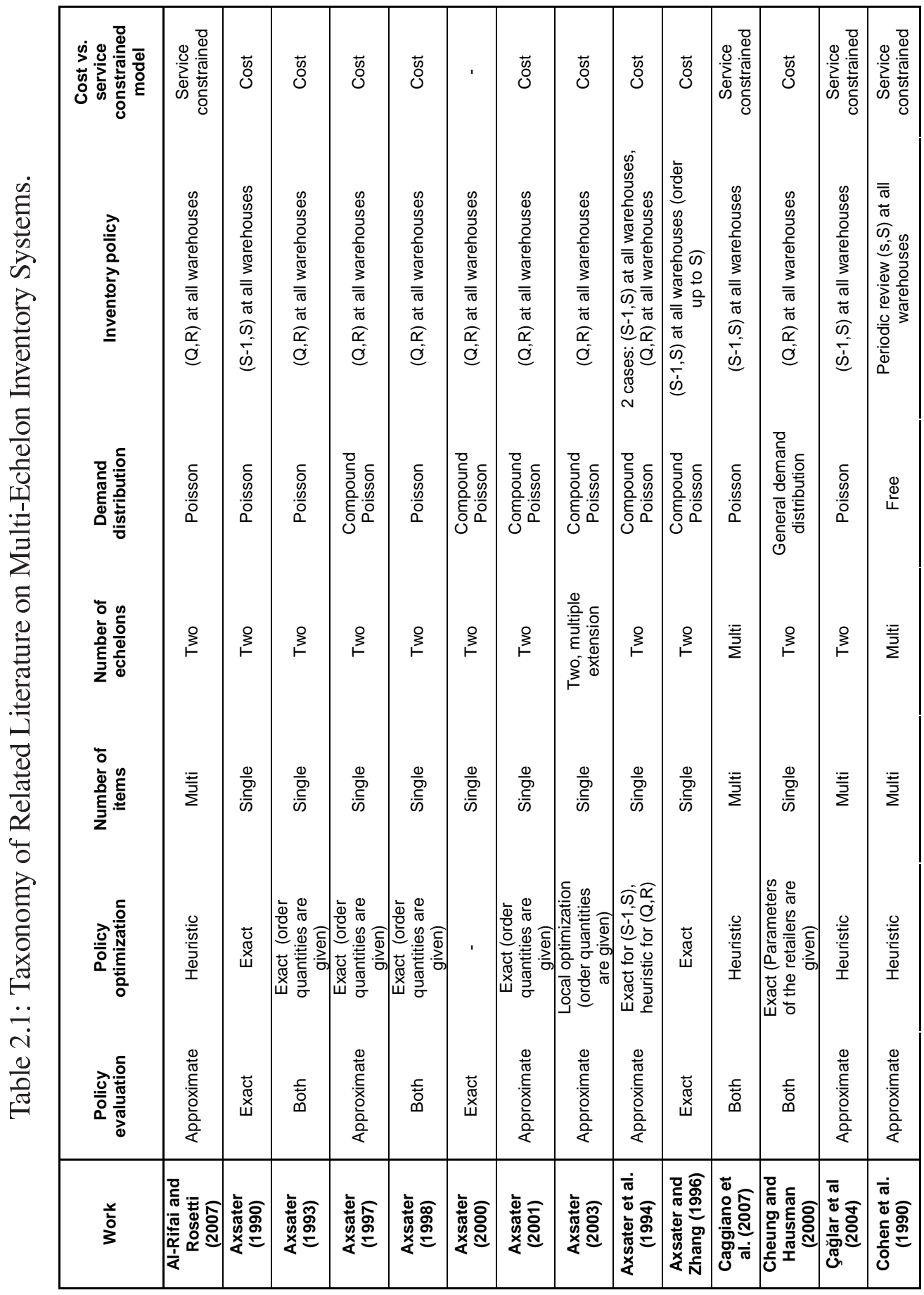




\begin{tabular}{|c|c|c|c|c|c|c|c|c|c|c|c|c|c|c|}
\hline 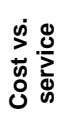 & 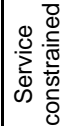 & $\begin{array}{l}\bar{m} \\
0 \\
0\end{array}$ & $\begin{array}{l}\bar{\Delta} \\
0 \\
0\end{array}$ & $\begin{array}{l}\bar{\delta} \\
0 \\
0\end{array}$ & $\begin{array}{l}\text { गे } \\
\text { Oे }\end{array}$ & $\begin{array}{l}\bar{w} \\
\text { Oे }\end{array}$ & 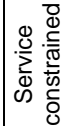 & 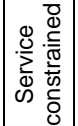 & 茄 & 范 & 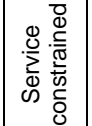 & 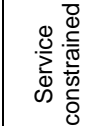 & , & $\begin{array}{l}\bar{m} \\
0 \\
0\end{array}$ \\
\hline 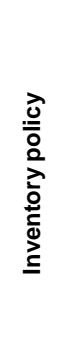 & 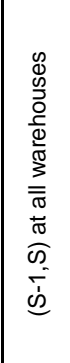 & 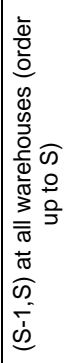 & 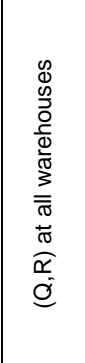 & 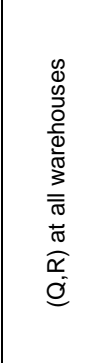 & 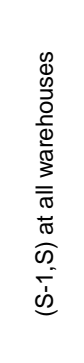 & 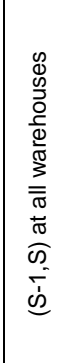 & 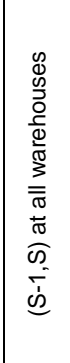 & 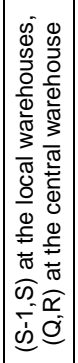 & 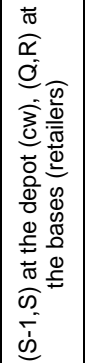 & 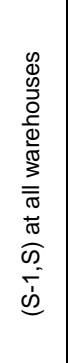 & 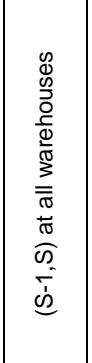 & 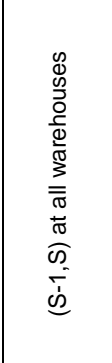 & 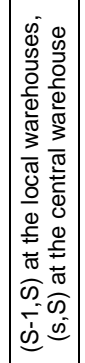 & 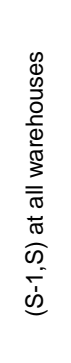 \\
\hline 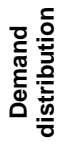 & 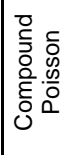 & 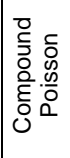 & $\begin{array}{l}\overline{.} \\
.0 \\
.00 \\
0.0 \\
0\end{array}$ & 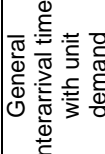 & 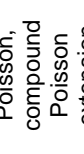 & 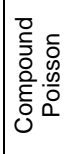 & 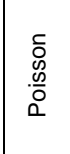 & $\begin{array}{l}\check{\delta} \\
.0 \\
\frac{0}{0} \\
0 \\
0\end{array}$ & 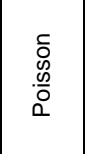 & $\begin{array}{l}\text { 대 } \\
.0 \\
.0 \\
0\end{array}$ & $\begin{array}{l}. \overline{0} \\
.0 \\
.0 \\
0 \\
0\end{array}$ & 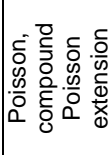 & 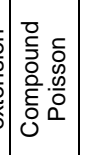 & 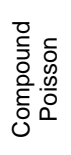 \\
\hline 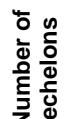 & $\frac{\bar{m}}{\overline{2}}$ & $\sum_{n}^{0}$ & $\stackrel{0}{\xi}$ & $\stackrel{0}{3}$ & 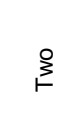 & $\sum_{\xi}^{0}$ & $\sum_{\xi}^{0}$ & $\sum_{\xi}^{0}$ & $\stackrel{0}{\xi}$ & $\stackrel{0}{\xi}$ & $\sum_{1}^{0}$ & $\sum_{n}^{0}$ & $\sum_{\xi}^{0}$ & $\stackrel{o}{\xi}$ \\
\hline 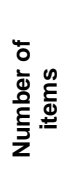 & 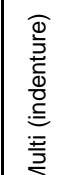 & $\begin{array}{l}\frac{0}{0} \\
\frac{5}{5}\end{array}$ & $\begin{array}{l}\frac{\omega}{\square} \\
\frac{5}{\omega}\end{array}$ & $\begin{array}{l}\frac{\omega}{0} \\
\frac{5}{\omega}\end{array}$ & $\begin{array}{l}\frac{0}{0} \\
\text { के } \\
\text { के }\end{array}$ & $\begin{array}{l}\frac{0}{0} \\
\frac{5}{5}\end{array}$ & $\frac{\text { 吾 }}{\sum}$ & $\frac{\mathrm{E}}{\mathrm{z}}$ & $\begin{array}{l}\frac{\omega}{\Phi} \\
\frac{5}{\omega}\end{array}$ & 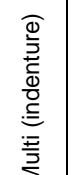 & $\frac{\bar{z}}{\bar{z}}$ & $\begin{array}{l}\frac{0}{0} \\
\frac{5}{i n}\end{array}$ & $\begin{array}{l}\frac{0}{\overline{9}} \\
\dot{\omega}\end{array}$ & $\begin{array}{l}\frac{0}{0} \\
\frac{5}{5}\end{array}$ \\
\hline 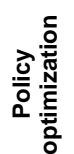 & 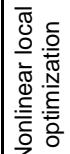 & 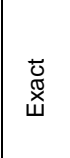 & 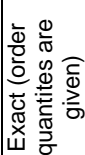 & & $\begin{array}{l}\text { Fे } \\
\text { Oे }\end{array}$ & ' & 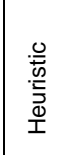 & $\begin{array}{l}\frac{0}{\bar{D}} \\
\frac{0}{\overline{5}} \\
\frac{0}{1} \\
\frac{0}{1}\end{array}$ & $\begin{array}{l}\frac{0}{\overline{7}} \\
\frac{0}{\overline{5}} \\
\frac{0}{1} \\
\frac{0}{1}\end{array}$ & 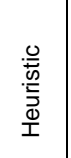 & 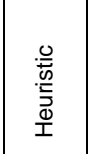 & 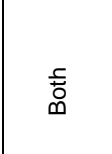 & & ' \\
\hline 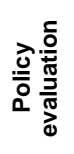 & $\begin{array}{l}\frac{0}{\pi} \\
\frac{\tilde{E}}{\frac{1}{x}} \\
\frac{0}{0} \\
\frac{0}{2}\end{array}$ & 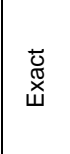 & 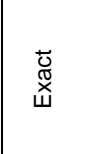 & 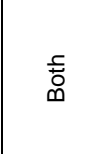 & 产 & $\begin{array}{l}\frac{0}{\pi} \\
\frac{\tilde{E}}{x} \\
\frac{0}{0} \\
\frac{0}{2}\end{array}$ & $\begin{array}{l}\frac{0}{\pi} \\
\frac{\pi}{\frac{\pi}{x}} \\
\frac{0}{0} \\
\frac{0}{2} \\
\frac{2}{4}\end{array}$ & 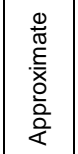 & $\begin{array}{l}\frac{0}{\pi} \\
\frac{\pi}{x} \\
\frac{0}{0} \\
\frac{0}{\frac{0}{2}} \\
\frac{0}{4}\end{array}$ & $\begin{array}{l}\frac{0}{\pi} \\
\stackrel{\frac{5}{x}}{\frac{0}{0}} \\
\frac{0}{0} \\
\frac{0}{<}\end{array}$ & $\begin{array}{l}\frac{0}{\pi} \\
\frac{\pi}{x} \\
\frac{0}{0} \\
\frac{0}{\frac{0}{2}} \\
\frac{1}{4}\end{array}$ & 言 & 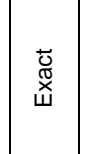 & $\begin{array}{l}\frac{0}{\tilde{\oplus}} \\
\stackrel{\frac{5}{x}}{\frac{0}{0}} \\
\frac{0}{0} \\
\frac{0}{x}\end{array}$ \\
\hline 总 & 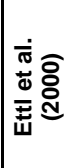 & 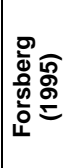 & 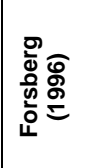 & 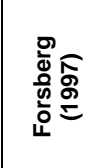 & 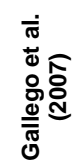 & 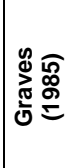 & 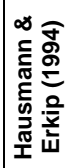 & 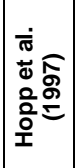 & 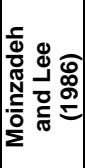 & 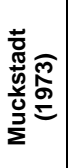 & 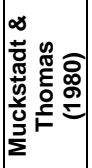 & 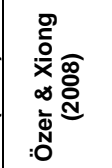 & 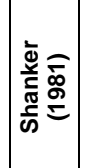 & 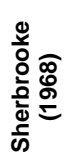 \\
\hline
\end{tabular}




\begin{tabular}{|c|c|c|c|c|c|c|c|c|c|}
\hline 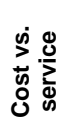 & 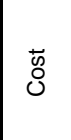 & & $\begin{array}{l}\text { t. } \\
\text { Oे }\end{array}$ & & 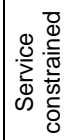 & 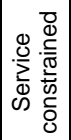 & 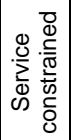 & 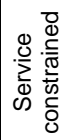 & 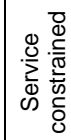 \\
\hline 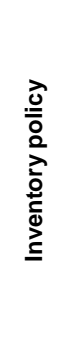 & 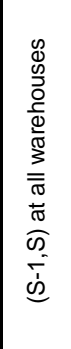 & 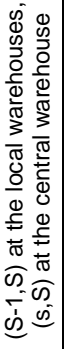 & 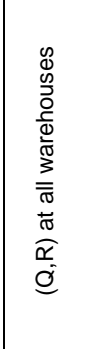 & 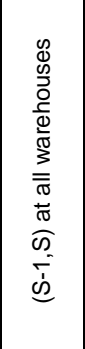 & 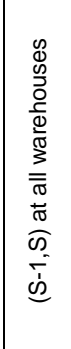 & 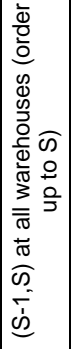 & 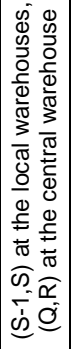 & 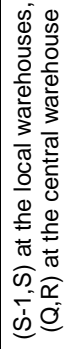 & 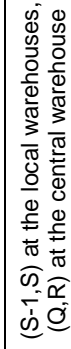 \\
\hline 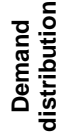 & $\begin{array}{l}\overline{0} \\
\overline{0} \\
.00 \\
0 \\
0\end{array}$ & 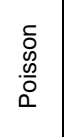 & 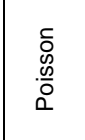 & $\begin{array}{l}\bar{\delta} \\
\text {. } \\
.00 \\
0 \\
0\end{array}$ & 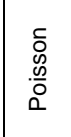 & 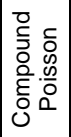 & $\begin{array}{c}\sigma \\
0 \\
.0 \\
0 \\
0 \\
0\end{array}$ & 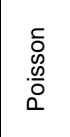 & 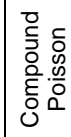 \\
\hline 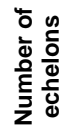 & $\sum_{\underline{n}}$ & $\stackrel{\rho}{\xi}$ & $\sum_{1}$ & $\frac{\mathrm{E}}{\bar{z}}$ & $\sum_{\underline{1}}$ & $\frac{\mathrm{E}}{\bar{z}}$ & $\stackrel{o}{\xi}$ & $\sum_{\underline{1}}$ & $\sum_{n}$ \\
\hline 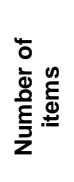 & 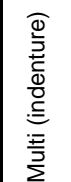 & $\begin{array}{l}\frac{0}{\bar{O}} \\
\stackrel{5}{5}\end{array}$ & $\begin{array}{l}\frac{0}{0} \\
\frac{10}{5}\end{array}$ & $\begin{array}{l}\frac{0}{0} \\
\frac{5}{\omega}\end{array}$ & $\frac{\bar{T}}{\bar{y}}$ & 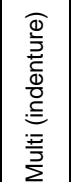 & $\frac{\bar{z}}{\bar{z}}$ & $\frac{\overline{\bar{z}}}{\sum}$ & 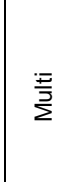 \\
\hline 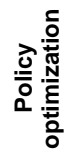 & ' & & 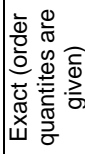 & & 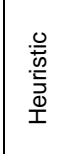 & \begin{tabular}{|c|c} 
\\
$\tilde{y}$ \\
w
\end{tabular} & 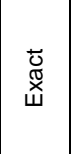 & 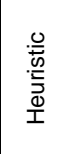 & 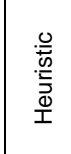 \\
\hline 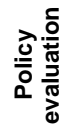 & 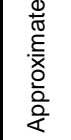 & $\begin{array}{l}\overline{\tilde{U}} \\
\text { ய. }\end{array}$ & 咅 & 喜 & 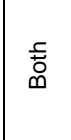 & \begin{tabular}{|c|c} 
\\
$\tilde{y}$ \\
w
\end{tabular} & \begin{tabular}{|c|} 
\\
$\tilde{y}$ \\
$\tilde{x}$
\end{tabular} & 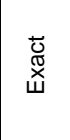 & 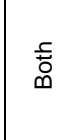 \\
\hline 今े & 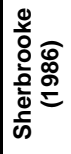 & 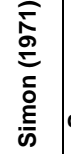 & 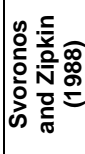 & 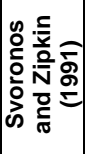 & 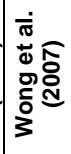 & 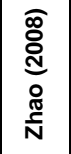 & 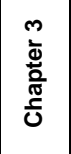 & 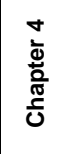 & $\begin{array}{l}\text { no } \\
\frac{0}{ \pm} \\
\frac{0}{\pi} \\
\frac{\pi}{0}\end{array}$ \\
\hline
\end{tabular}




\subsubsection{Single-Item Systems}

One of the seminal works in this area is proposed by Sherbrooke (1968). He proposes an approximate model called METRIC for two-echelon repairable inventory distribution system consisting of a number of identical bases and a depot. All facilities operate under a base-stock policy. The system faces a compound Poisson demand and the repair times are stochastic. The objective is to find the base-stock levels minimizing the expected number of backorders subject to a budget constraint. In METRIC, Sherbrooke (1968) replaces the stochastic repair (lead) time at the bases by its mean to approximate the probability distributions of the number of inventory and backorder levels at the bases. This simplifies the derivation of the system-related performance measures, e.g., the number of outstanding orders at the bases has a Poisson distribution. Furthermore, this brings a significant computational advantage for the method. Therefore, the METRIC becomes one of the most common approximation used for multi-echelon inventory systems. Although the model is developed for single-item systems, it is adopted in multi-item setting by many researchers (Çăglar et al. 2004, Wong et al. 2007b). Nevertheless, since the METRIC ignores that the replenishment lead time and the average delay at the bases are dependent on the inventory level at the depot, the number of backorders is underestimated and this results in highly infeasible solutions (Graves 1985, Wong et al. 2007b). This is the main disadvantage of the METRIC. Sherbrooke does not consider the optimization problem of the system.

Muckstadt (1973) extends Sherbrooke's METRIC to a multi-item multi-indenture setting by proposing an approximation called MOD-METRIC. He considers the same problem structure with Sherbrooke (1968) but in a multi-item and multi-indenture setting, e.g., a two-echelon repairable inventory distribution system operating under a base-stock policy and the objective is to find the base-stock levels minimizing the expected number of backorders. The only difference is that, in this model, a Poisson demand is assumed. Although the main focus of the METRIC is the policy evaluation, in MOD-METRIC, Muckstadt (1973) also focuses on the policy optimization issue and proposes a heuristic search method to find the base-stock levels of the system. Similar to the METRIC, the MOD-METRIC underestimates the expected backorder levels. 
Graves (1985) considers a multi-echelon inventory distribution system for repairable items consisting of a repair depot and a number of nonidentical operating sites under a base-stock policy. He assumes a compound Poisson demand. To find the base-stock levels, he proposes an exact evaluation method for the system and derive the stationary distribution of the inventory and the backorder levels at all facilities. For the exact evaluation he uses an earlier result by Simon (1971), which we call the binomial disaggregation, which suggests that given the number of backorders at the depot, a backorder emanating from a certain site follows a binomial distribution. He also proposes an approximate evaluation method for the system based on a two-moment approximation. Accordingly, the distribution of the outstanding orders is approximated by fitting a two-parameter family of distribution, i.e., a negative binomial distribution. The approximate model is only implemented for the pure Poisson demand model. The performance of the approximation is tested against the METRIC. The author reports that the two-moment approximation is superior to the METRIC, i.e., the solution obtained by using the METRIC differ from the optimal solution at least one unit in $11.5 \%$ of the problem instances, while that of obtained by using his method is only $0.9 \%$. This shows that the understocking problem of the METRIC is solved up to a certain level by using the two-moment approximation, which requires keeping more stock than the METRIC. The author also reports that the computational requirements of the two methods are comparable. These results make Graves' two-moment approximation a commonly used evaluation method for multi-echelon inventory distribution systems. Similar to the METRIC, it is also widely used in multi-item systems (Hopp et al. 1999, Wong et al. 2007b).

Sherbrooke (1986) considers the same setting as Muckstadt (1973). Similar to the manner the METRIC is improved by Graves (1985), he proposes a two-moment approximation for the number of outstanding orders, i.e., units in supply or in repair to improve the MOD-METRIC. Through a simulation study, he shows that the expected backorder level estimations are close to the simulated values. He also states that the performance of the two-moment approximation improves further compared to the METRIC in multi-item systems compared to the single-item. In this sense, Sherbrooke (1986) extends the earlier findings obtained by Graves (1985) for single-item systems to multi-item ones. Sherbrooke (1986) deals only with the policy evaluation 
issue, hence, does not provide a solution procedure to find the base-stock levels.

Ettl et al. (2000) consider a multi-echelon inventory and production distribution and assembly system, facing a compound Poisson demand. All facilities operate under an $(S-1, S)$ policy. The objective is to minimize the expected cost of on hand and WIP inventory subject to service level constraints based on the time-based fill rates. They propose an approximate evaluation method and a heuristic procedure to find the policy parameters. The approximation is a queueing-based approximation for the system to estimate the replenishment lead time (the sum of the transportation lead time and the delay due to stockout). For this purpose, the number of jobs in the retailers, each of which is modelled as $M^{x} / G / 1(M / G / 1$ with compound Poisson arrivals) queueing system, are approximated by a normal distribution. For the optimization of the system, they use a conjugate gradient method to solve the nonlinear objective function. The algorithm iterates by computing the gradients until the value of the objective function converges. They test the performance of the entire method using simulation. The simulation runs show that both the approximation and the heuristic perform quite well. They report that the method can be used in large complex supply chain networks.

Svoronos and Zipkin (1991) consider a more complex tree-structure multi-echelon inventory distribution system having a warehouse and a number of nonidentical retailers, all operating under a base-stock policy. They assume that the demand is Poisson, transposition lead times are stochastic. In order to analyze the entire system, first they study the single-facility problem and characterize the exact distributions of the outstanding orders, inventory and backorder levels for this system. Later, they extend these results to general multi-echelon systems. They also propose an approximation for the steady-state distribution of the number of inventory and the backorder levels for given base-stock levels. Their method relies on applying a two-moment approximation by fitting a negative binomial distribution to the distribution of the outstanding orders at the retailers.

In a recent study, Gallego et al. (2007) consider a two-echelon distribution system consisting of a warehouse and a number of nonidentical retailers. All facilities operate under base-stock polices. The demand is Poisson, although a compound Poisson 
extension is considered as well. The objective is to minimize the average inventory and backordering costs. In this paper, they evaluate the performances of the local control, i.e., installation stock policies, and the central control, i.e., echelon stock policies. In order to analyze the system behaviour under the local control, they use the exact analysis developed by Simon (1971) and Graves (1985). They also propose two approximations. The first one is the normal approximation for the demand, while the second uses a distribution-free bound to approximate the average cost function. In order to optimize the policy parameters, they consider an exact solution procedure similar to the one developed by Axsäter (1990) and a heuristic method. For the latter one, they apply three simple subheuristics. First one assumes that the warehouse does not hold stock, while the second one assumes that the warehouse holds maximum stock. Third one assumes that the warehouse holds inventory but does not hold safety stock. Among all these subheuristics they select the best. In this paper, they also consider the compound Poisson demand case. For the compound Poisson demand setting, they approximate the overshoot due to demand sizes at the retailers using a two-moment approximation. They apply the two-moment approximation for the distribution of backorders emanating from retailers, which is binomially distributed in the Poisson demand case. To estimate the variance of the corresponding random term, they distribute the overshoot among the retailers as they distribute the demand. Through a computational study, the performances of the local and the central controls are compared. They find that the local control performs better when the holding cost at the warehouse, the penalty cost and the demand rates are low, the lead time for the warehouse is shorter relative the lead time at the retailers. Finally, they show that the central control is superior to the local control, however the results highly vary. They also test the performance of the approximations and the heuristics are tested against the exact evaluation and the optimization methods, respectively. Accordingly, the gap between the results obtained by using the exact method and that by the approximations is quite large. The percentage cost penalty of using the heuristic is $1.23 \%$ on the average. The heuristic is reported to be more accurate when the warehouse lead time is shorter. They also show that the heuristic is asymptotically optimal in the number of warehouses. For the performance of the approximation that is considered for the compound Poisson demand, they show that the corresponding approximation yields better results when the demand size distributions at different retailers are sim- 
ilar, and the overshoot is a small part of the total number of backorders at the central warehouse.

Özer and Xiong (2008) extend Gallego et al. (2007) to a similar system with service level constraints. The objective is to minimize the average inventory costs subject to fill rate constraints. For the evaluation of the system, both exact and approximate methods are considered. For the approximate evaluation of the system, they consider two different alternatives. The first one is a normal approximation of the demand at the retailers. The second one extends the distribution-free approximation proposed by Gallego et al. (2007) to their system setting. To find the optimal policy parameters, they propose an exact algorithm similar to Axsäter (1990). However, this algorithm, at each iteration, also checks whether the service level constraints are satisfied or not. They also consider two alternative heuristics to obtain the policy parameters. First one is the extension of the heuristic proposed by Gallego et al. (2007) to their system. The second heuristic decomposes the multi-echelon system to two-location serial inventory systems. They also consider extensions of the methods to compound Poisson demand, general multi-echelon tree structure distribution systems as well as multi-item systems with aggregate service measures. Through a computational study, the authors show that the average percentage cost penalty of using the first heuristic is less than $1.2 \%$. It is also reported that the first heuristic performs better when the lead time are shorter and the holding costs are lower. For the second heuristic, the average percentage cost penalty is found to be quite high, although it is computationally much faster than the former. The algorithms are reported to be quite efficient in terms of the computational requirements.

Apart from the approximations for base-stock systems in the literature, there are approximations proposed to evaluate the multi-echelon inventory distribution systems with batch ordering policy. A common approach among these papers is to approximate each facility as a single-facility inventory system. In one of these works, Svoronos and Zipkin (1988) consider a two-echelon inventory system consisting of a number of identical retailers and a warehouse replenishing them. They assume that all facilities operate under a $(Q, R)$ policy and demand is Poisson. The objective is to minimize the average inventory holding and backordering costs. In order to approximate the average inventory and backorder levels at the warehouse and the retailers, 
they decompose the problem and solve the problem of each facility separately. For the analysis of the warehouse, they consider both an exact and an approximate method. They approximate distribution of the demand during replenishment lead time, i.e., the sum of transportation time and delay at the central warehouse due to backorders, by using two-moment approximations, using three alternative two-parameter family of distributions. This simplifies the estimation of the average inventory and the backorder levels at the central warehouse. For the analysis of the retailer, first they approximate the probability distribution of the replenishment lead time at the retailer using a two-moment approximation based on a negative binomial distribution. Then, by using this approximate distribution and the variance estimates for backorders at the warehouse, they approximate the steady-state distributions of the outstanding orders and the inventory and the backorder levels at the retailers. In this way, these probability distributions are obtained by considering the dependencies between the facilities. Through a computational study, the performance of their method is tested against those of Deuermeyer and Schwarz (1981) and Lee and Moinzadeh (1984a, b), who study the same problem, by simulating the solutions obtained by each method. The simulation results show that their method is more accurate than the other methods. Although the main focus of the paper is the evaluation of the system, they also study the optimization problem of this system. They assume that the order quantities at all facilities are predetermined. To find the optimal reorder levels given the order quantities they use a simple search procedure based that given the reorder levels at the warehouse, for each retailer, the resulting cost function is convex in the reorder level.

Moinzadeh and Lee (1986) consider a two-echelon inventory system for repairable items. The system consists of a number of site and a depot replenishing them. All sites operate under a $(Q, R)$ policy, whereas the depot operates under an $(S-1, S)$ policy. In this sense, their system is exactly the opposite of our setting. They assume that the order quantity is identical for all sites, while the reorder levels are nonidentical. The objective is to minimize the average inventory holding, backordering and setup costs. To obtain the optimal or near-optimal policy parameters for the system, they propose a 2-step procedure. In the first step, the order quantity for the retailers is obtained by using a power approximation in which the average total cost is approximated as a 
nonlinear function of the order quantity. To obtain such an expression, a regression fit is performed by using 600 problem instances. In the second step, after determining the order quantity using the power approximations, the reorder levels at the sites and the depots are obtained by using a one-pass heuristic search procedure. That is, first for a given value of the reorder level at the depot, the optimal reorder levels at the sites are obtained. In this situation, the resulting average cost function is convex. Hence, it is quite simple to obtain the reorder levels at the sites. Then, after finding these parameters, a search for the optimal reorder level at the depot is made. In this case, the total cost function is not necessarily convex. However, one-pass heuristic iterates as if it is convex. The whole procedure also involves approximating the number of outstanding orders at each site by using a two-moment approximation by Lee and Moinzadeh (1984a). They show that the entire procedure gives the optimal solution in $95 \%$ of the instances. The method is also used to test whether the $(S-1, S)$ policy is optimal or not for repairable items. They show that when demand is low, the number of sites is high and average transportation or repair time is long, $(S-1, S)$ policy is more likely to be optimal.

Cheung and Hausmann (2000) study a two-echelon inventory system consisting of a supplier and a number of nonidentical retailers. The objective is to minimize the average inventory holding, backordering and setup costs. All facilities operate under a $(Q, R)$ policy. A general demand distribution is assumed. The authors show that the distribution of the inventory position is independent of the distribution of the inventory position at other facilities and as well as the lead time demand distribution at the supplier and also show that this distribution is uniform. This simplifies the derivation of the average inventory and backorder levels. Therefore, this makes it possible to have a closed form expression for the backorder levels at the supplier. They assume that retailer parameters are given. In this situation, the problems reduces to single-echelon $(Q, R)$ policy, hence, since the average total cost function is jointly convex in the reorder level and the order quantity, it becomes relatively easy to obtain the policy parameters. They also test the performance of using the Poisson demand approximation, which is quite often used in the literature. The results show that the performance of Poisson approximation is well but using it in the optimization of a two-echelon system may result in high loses. 
There are also papers studying the batch ordering problem of two-echelon inventory distribution systems facing a compound Poisson demand. Axsäter et al. (1994) consider such a system consisting of a central warehouse and a number of nonidentical retailers facing a compound Poisson demand. They propose an approximate evaluation method to analyze this system assuming that all facilities operate under a basestock policy. They also consider the extension of the method to batch ordering. In this method, the compound Poisson demand model is replaced by a Poisson demand model such that the mean to variance ratio is preserved. In this way, they also question whether a Poisson demand model can be used although the demand is not Poisson. After replacing the compound Poisson demand model, the policy parameters of the Poisson demand model is obtained by using a heuristic similar to Moinzadeh and Lee (1986). They also consider the optimal solution of the policy parameters using the method proposed by Axsäter (1990) (Axsäter's paper is reviewed under the exact evaluation methods) for two-echelon systems facing Poisson demand. The optimal solution is used to test the performance of the approximation as well as the heuristic. For the batch ordering model, since order quantities are assumed to be given, hence, the algorithms are extended to the batch ordering problem easily. Through a computational analysis, the solution is found to be very close to optimum. However there are variations in the performance of the method, e.g., in some of the problem instances the method is outperformed by another method, probably a worst possible alternative, in which Poisson model is directly used without any scaling. The average percentage cost penalty of using this model is found to be about $1 \%$.

Axsäter et al. (1997) consider a two-echelon inventory distribution system consisting of a central warehouse and a set of nonidentical retailers. All facilities operate under a $(n Q, R)$ policy. The demand is assumed to be compound Poisson. The objective is to minimize the system-wide inventory holding and shortage costs. The author proposes an approximate method to evaluate the system-wide cost. The method is based on two approximations. First, the correlation between the number of backorders from retailers at the central warehouse and the outstanding orders at other retailers are ignored so that each retailer is considered separately. The errors due to the first approximation is found to be negligible. Second, Poisson demand process is discretized by using multiple discrete time steps. To expedite the procedure, he limits the number of time 
steps and implements a linear extrapolation to estimate the system-wide cost as function of the number of time step. Through a computational study the author shows that the relative error between the exact cost and the approximate cost is less $0.43 \%$. The author also proposes an exact solution procedure for the optimization of the policy parameters. Since the order quantities are assumed to be given, to find the optimal reorder levels at the central warehouse and the retailers he extends the exact solution procedure proposed by Axsäter (1990) to compound Poisson setting.

Axsäter (2001) considers a system with a central warehouse and a number of nonidentical retailers system, where all facilities operate under a $(n Q, R)$ policy and demand is compound Poisson. The objective is to minimize the average inventory holding, backordering costs. He focuses on systems with high demand and develops an approximation for such systems by approximating the high demand case with a low demand case. First, the demand is scaled down such that the variance-to-mean ratio is preserved. Then, the system is optimized based on the low demand model. He assumes that the order quantities are determined in advanced. The reorder levels are optimized by using a method similar to Moinzadeh and Lee (1986). After finding the optimal parameters of the low demand system, the solution of the low demand model is transformed back to the high demand case. This step requires scaling down the average demand, order quantities and reorder levels by the same factor. Since the method requires scaling down the reorder level, order quantities by a common factor, it is only suitable for high demand models, e.g., it is not an appropriate model for systems in which the optimal order quantity for an item could be 1 such as the one considered in this dissertation. Since the exact evaluation of the system is intractable, they consider simulating the system. The simulation runs show that the percentage cost penalty of using the method is around $2.0 \%$ but increases as the demand size increases.

Axsäter (2003) considers a system with a central warehouse and a number of nonidentical retailers system. The demand is assumed to be compound Poisson. The objective is to minimize the average inventory holding, backordering costs. All facilities apply a $(n Q, R)$ policy. He approximates the lead time demand distribution using a normal distribution. Similar to Axsäter (2001), order quantities are assumed to be given or predetermined. In order to find the reorder levels, similar to Moinzadeh 
and Lee (1986), a heuristic procedure is used based on the assumption that the cost function is convex in reorder level at the central warehouse. He also considers a lower and an upper bound to expedite the procedure. Later, the method is extended to systems with more than two-echelons as well. The method is suitable for large systems, hence, tractable for practical size systems. The percentage cost penalty of using the method is $0.6 \%$, which is quite satisfactory.

Forsberg (1997) considers a two-echelon inventory system with a central warehouse and a number of retailers. All facilities operate under a $(Q, R)$ policy. This paper differs from the other papers in that the customer inter-arrival times are assumed to be generally distributed and the demand is assumed to be unit demand. The author only considers the policy evaluation issues. First he extends the exact evaluation method proposed by Forsberg (1996) to this setting. He also proposes two different approximations each of which is based on approximating the generally distributed inter-arrival times by an Erlang distribution. The performance of the approximations are tested against the simulation runs. The two approximation methods give the same results in all instances. The average relative difference between the simulated and the approximated values for the average inventory and backorder levels is reported to be $1.5 \%$. Nevertheless, he also reports that there are variations in the results.

Although the approximations are prevalent in the literature, using them in a policy optimization problem for a multi-item system under service level constraints may result in infeasible solutions. In this situation, the measure of the infeasibility of the solution obtained by a certain approximation becomes very important. The two most commonly used approaches in multi-echelon inventory distribution systems, i.e., the METRIC and the two moment approximation, are tested in a single-item (Graves 1985) and a multi-item setting (Wong et al. 2007) and it is shown that the performance of the Graves's two-moment approximation is quite well, whereas the METRIC may yield highly infeasible solutions. Therefore, whether one can use an approximation depends on the performance of the corresponding approximation. Although the performance of the commonly used approximations are tested in single-item multiechelon inventory distribution systems facing Poisson demand, their performances are not investigated under a multi-item, compound Poisson demand setting. In this dissertation, in Chapter 5, we consider several approximations for the analysis of the 
system under the compound Poisson demand setting. Three of these approximations correspond to the extensions of the METRIC and the two-moment approximations to our setting. We provide a comparison of these approximations and the results of this comparison contributes to the relevant literature. As opposed to the approximations, exact methods guarantee feasible solutions when they are employed to find the optimal or near-optimal policy parameters.

There exists exact evaluation methods proposed for multi-echelon inventory systems as well. Although the main contribution of Graves (1985) is the proposal of the twomoment approximation for base-stock systems, he also proposes an exact evaluation method for multi-echelon inventory distribution systems. Therefore, this work also belongs to the stream of literature that propose exact evaluation methods proposed for multi-echelon inventory systems. The method relies on the lead-time demand method and utilizes the binomial disaggregation.

Axsäter (1990) considers a two-echelon inventory system with a warehouse and a set of retailers. All facilities operate under a base-stock policy. The demand is Poisson. The objective is to minimize the average inventory holding and backordering costs. In this seminal work, Axsäter develops both an exact evaluation and an exact optimization algorithm for the system. The exact evaluation method is based on keeping track of each unit as it moves through the system, which is known as flow-unit method (Simchi-Levi and Zhao 2010). The method facilitates directly evaluation of the average system-wide cost function for a given base-stock policy. In this sense, it differs from the lead time demand method, which requires determining of the stationary distribution of the inventory and the backorder levels (Simon 1971 and Graves 1985). The exact optimization algorithm is summarized as follows. First, for a given base-stock level at the warehouse, the base-stock levels at the retailers are obtained. In this situation, the entire problem decomposes into single facility problems, hence, obtaining the solution for these subproblems becomes quite simple since the cost function is convex. After finding the solutions of all subproblems, the system-wide cost function is optimized over the base-stock level at the warehouse. This requires an exhaustive search since the function is not necessarily convex in base-stock level at the central facility. However, to expedite the procedure, he proposes lower and upper bounds on the base-stock level at the warehouse. After this seminal work, many of 
the papers published use this exact method or a similar one to search for the optimal solution of the two-echelon inventory distribution systems. In this dissertation, we consider a similar procedure to solve single-item subproblems, which is later used in the development of the exact solution procedure and the heuristics for our problem. Our algorithm differs from Axsäter (1990) in that it is proposed for a batch ordering system.

There are exact evaluation methods proposed for batch ordering systems as well. In one of the seminal works, Simon (1971) extends Sherbrooke's METRIC to a different setting allowing the analysis of consumable, repairable and condemned parts together. However, he assumes a Poisson demand and constant repair (lead) time. Similar to our system setting, he assumes a batch ordering at the depot, a base-stock policy at the bases. The difference from our system setting is that the depot operates under an $(s, S)$ policy. He deals with the exact analysis of the system and derives the steadystate probability distributions of the inventory and the backorder levels at all facilities and as well as the number of items in repair. The method is based on the lead time demand method, in which the distributions of the inventory and the backorder levels are obtained based on the lead time demand distribution (Simchi-Levi and Zhao 2010). His findings are seminal and used by many other researchers for the analysis of two-echelon inventory distribution systems with different settings, e.g., he shows that binomial disaggregation is exact for two-echelon inventory distribution systems under Poisson demand.

Axsäter (1993) considers a two-echelon inventory system with a central warehouse and a number of identical retailers. All facilities operate under a $(Q, R)$ policy. The demand is Poisson. He proposes to express the system-wide cost under the $(Q, R)$ policy as a weighted average of system-wide costs under base-stock policies. He shows that, in this situation, many of the results obtained for base-stock policies by Axsäter (1990) can be extended to batch ordering problem. His method relies on the flow-unit method, in which a single unit is followed. In this method, first he introduces two probabilities, i.e., $p_{i j}$, probability that $i^{\text {th }}$ subsequent system demand will trigger the $j^{\text {th }}$ subsequent retailer order, and $q_{m j}$, the probability that $m$ demands arrive at the retailer during the waiting for the $j^{\text {th }}$ subsequent retailer order. Then he defines the entire cost function based on these probabilities. First, he proposes an exact evalua- 
tion method to calculate these probabilities, as well as, the system-wide cost function. However, since the exact evaluation is computationally prohibitive, he proposes three alternative approximations for $p_{i j}$. Since the method proposed by Svoronos and Zipkin (1988) outperforms those of Deuermeyer and Schwarz (1981) and Lee and Moinzadeh $(1984 a, b)$, he compares the performances of the approximations with that of Svoronos and Zipkin (1988). The results show that third approximation is slightly more accurate than that of Svoronos and Zipkin (1988). For the optimization of the system, he assumes that order quantities are predetermined.

Axsäter (1998) extends the analysis of Axsäter (1993) to a similar system with nonidentical retailers. First, he proposes an exact evaluation method for the two retailer case. Then using this model, he approximates systems with more than two retailers. In latter case, when a retailer is analyzed, the other retailers are aggregated into a single dummy retailer so that the entire problem becomes a two-retailer problem. Since the sum of Poisson processes is also Poisson process, the method is quite reasonable for Poisson demand. For the optimization problem, he assumes that the order quantities are predetermined. Then the reorder levels are optimized using an exact search procedure similar to Axsäter (1990).

In another related work in this steam, Forsberg (1996) considers a two-echelon system with one warehouse and a number of nonidentical retailers. Just like Axsäter (1993), he proposes an exact evaluation method for two-echelon systems with batch ordering policy. Just like Axsäter (1993), the average system-wide cost for this batch ordering system is expressed as a weighted average of base-stock costs. Therefore, the average cost is obtained by using the results in Axsäter (1990). Through a computational study, the performance of the exact method is tested with the extension of Svoronos and Zipkin's (1988) to nonidentical retailers. In this study, as in many of the papers studying the batch ordering problems, he assumes that order quantities are given.

The methods proposed by Axsäter (1993), Axsäter (1998) and Forsberg (1996) rely on the flow-unit method, which is based on keeping track of each unit as it moves through the system. The main disadvantage of their method is that they require recalculation of the expressions and the convolution when the order quantities at the retailers change. Therefore, the methods is not suitable when the system is optimized 
with respect to the order quantities at the retailers.

There are also studies proposing exact evaluation methods for systems facing compound Poisson. However, compound Poisson demand introduces many challenges to the evaluation of multi-echelon inventory distribution systems. First, the binomial disaggregation fails, hence, exact evaluation methods based on lead-time demand method become quite complicated (Shanker 1981). Similarly, the flow-unit method, which is involved even for Poisson demand, still works but becomes more involved (Axsäter 2006). Shanker (1981) considers a two-echelon item inventory distribution system in which items can be consumable, repairable and condemned. The system consists of a set of bases and a central depot replenishing them. The bases operate an $(S-1, S)$ base-stock policy and the depot applies an $(s, S)$ policy. Hence, just like in our system, the author considers batch ordering only at the central warehouse, but, with a different batch ordering policy. The demand is assumed to be compound Poisson. For the corresponding system, he derives the exact steady-state distributions of the inventory position at the central warehouse and show that it is uniform. He also obtain the exact steady-state distributions of the inventory and the backorder levels at all facilities. The author only deals with the evaluation of the system. He does not provide a solution method for the problem. Instead, he indicates that the exact evaluation method can be used to obtain a total cost function as well as the system performance measures and all these later can be used in the optimization of the overall systems. However, he argues that this is quite computationally complicated since the method requires computation of several expressions and convolutions.

Forsberg (1995) analyzes a two-echelon system with one warehouse and a number of nonidentical retailers. He assumes a compound Poisson demand. All facilities operate under an order-up-to-S policy, which is the extension of $(S-1, S)$ base-stock policy to compound Poisson demand. He proposes an exact evaluation method to obtain the average system-wide inventory holding and backordering costs. The method relies on replacing the compound Poisson demand with a Poisson demand. He expresses the average system-wide cost as a weighted average of system-wide costs for the Poisson demand case and solves the Poisson demand case using the method proposed by Axsäter (1990). To optimize the problem he extends the search algorithm proposed by Axsäter (1990) to compound Poisson setting. 
Axsäter and Zhang (1996) study a two-echelon inventory system with one warehouse and a number of nonidentical retailers. The demand is compound Poisson. All facilities operate under an order-up-to-S policy. The objective is to minimize the average inventory holding and shortage costs. They provide a recursive procedure for the evaluation of this system by extending the exact evaluation method proposed by Axsäter (1990) to a compound Poisson demand setting. Although Forsberg (1995) derives the exact distribution for the compound Poisson case by solving several Poisson demand models, Axsäter and Zhang (1996) derive the cost function directly. To optimize the systems, they consider the algorithm in Axsäter (1990). Just like other papers on batch ordering policies, they optimize the system parameters assuming that the order quantities are determined in advance.

The exact evaluation of batch ordering systems with $(Q, R)$ policy under a compound Poisson setting is considered only by Axsäter (2000). In this paper, Axsäter (2000) considers a two-echelon inventory system having a warehouse and a number of nonidentical retailers. The demand is compound Poisson. To evaluate the system, he proposes an exact evaluation method, by extending the earlier methods based on the flow-unit method. First, the steady-state probabilities of the inventory and the backorder levels at the retailers are obtained. Then, using these probabilities, the average inventory and the backorder levels are obtained. Finally, the average inventory and the backorder levels at the central warehouses is obtained by using the average inventory and the backorder levels at the retailers. As opposed to earlier studies, e.g., Axsäter (1998), that are based on deriving the average cost function without finding these steady state probabilities of inventory and backorder levels, this method requires evaluating the steady-state probabilities of the inventory and the backorder levels directly. Hence, the main advantage of the method is that it is applicable to systems with any type of cost structure. Also in this method, system-wide cost is directly generated for the compound Poisson demand, whereas in Forsberg (1995) they are obtained from the Poisson demand costs and this makes the method more efficient than the latter. Nevertheless, the procedure is not tractable for problems with large demands and large order quantities. Therefore, he suggest a method making it possible to replacing the high demand system with a low one similar to the one discussed in Axsäter (2001). The method is quite tractable when the order quantities are between 
1 and 10, but becomes involved as they increase, especially when order quantities are optimized as well. As in many of the papers on batch ordering policies, order quantities are assumed to be predetermined. The exact analysis of the compound Poisson demand model that we provide in Chapter 5 mainly relies on the method developed by Axsäter (2000).

In a recent work, Zhao (2008) considers a more complex supply chain structure in which there is at most one directed path between stages. This system structure involves assembly, distribution systems, tree structure distribution systems as well as two-echelon distributions systems. The demand is compound Poisson. All facilities operate under a base-stock policy. He provides an exact and an approximate evaluation for the analysis of the system. The author argues that method developed by Forsberg (1995) and Axsäter (2000) are computationally more efficient than this method. The advantage of Zhao's method is that it is a more generic one and can be applied to various, more general supply chain systems. To find the optimal basestock polices they consider a method based on a dynamic programming (DP) based algorithm originally developed by Simchi-Levi and Zhao (2005).

\subsubsection{Multi-Item Systems}

Apart from studies on single-item multi-echelon inventory distribution systems, a more recent- body of papers study the multi-item systems. We mainly contribute to this body of the literature. Mostly, these papers propose heuristics to obtain the policy parameters of the multi-item multi-echelon inventory distribution systems. Therefore, solving practical industry-size problems is critically important. A common approach is to decompose the problem by facilities and/or parts, predominantly by means of a Lagrangian relaxation, then apply an iterative procedure to combine the resulting subproblems, which is also the case in our work. Also, most of the papers rely on approximations, although there are heuristics based on exact evaluation methods as well. Recall that in our study, we consider exact evaluation for the Poisson demand, whereas for the compound Poisson demand we consider both methods. Just like the one in this dissertation, the system approach is common among all these papers.

In an earlier work, Muckstadt and Thomas (1980) consider a multi-item two-echelon 
inventory system having a distribution center and a number of customer-service warehouses. All facilities operate under an $(S-1, S)$ policy. The demand is Poisson. The authors investigate the advantage of multi-echelon inventory systems over singleechelon model, hence, compare the performance of the single-echelon model with that of the multi-echelon model. In the single-echelon model, the base-stock levels are determined separately for each echelon and location, where the objective is to minimize the inventory investment subject to an aggregate service level constraint. In the multi-echelon model, the base-stock levels at different locations are determined simultaneously, where the objective is to minimize the expected replenishment lead time for a customer demand subject to budget constraint. To approximate expected replenishment lead time, they consider an approximation used in a earlier study due to Feeney and Sherbrooke (1966). Both models are solved by using a Lagrangian relaxation of the constraints. They report that the single-echelon model requires twice as much inventory as the multi-echelon model. For low demand and high cost items, the multi-echelon model is much more suitable. The computational requirements of the multi-echelon model is comparable with that of the single-echelon model. Hence they conclude that it is worth to use multi-echelon models.

Hausmann and Erkip (1994) extend the results of Muckstadt and Thomas (1980). Similarly, they compare the multi-echelon model and the single-echelon model in which the latter is used to approximate the former. They propose an iterative search procedure to find the base-stock levels. They find the single-echelon model is more appropriate when the budget constraint is less tight. They report that the percentage cost penalty of using single-echelon models is less than $5 \%$.

Çağlar et al. (2004) study a two-echelon spare parts inventory distribution system consisting of a central warehouse and a number of field depots. All facilities implement base-stock policies. The demand is Poisson. The objective is to minimize the system-wide inventory cost subject to an aggregate mean response time constraint. The authors propose a heuristic to find the policy parameters. First, they decompose the problem by echelons, each of which is solved using the METRIC approximation due to Sherbrooke (1968). Then, they apply an iterative procedure to combine the subproblems. The experimental study shows that their heuristic yields more accurate results than that of Hopp et al. (1999). 
Wong et al. (2007b) study the same problem as Çağlar et al. (2004). He propose four different heuristics to find the optimal base-stock levels for a two-echelon pure basestock system. The heuristics are based on various combinations of a greedy heuristic, a local search and the decomposition and column generation (DCG) method. Through extensive experiments with problem instances up to a size of 100 parts and 20 local warehouses, the performances of the heuristics are tested against a lower bound proposed in the study. They report that the greedy heuristic combined with the decomposition and column generation (DCG) yields quite satisfactory results in their setting, but the heuristic is tractable for problems up to a size of 100 parts and 20 local warehouses. They report that the greedy heuristic combined with DCG yields quite satisfactory results, with an average relative gap less than $2 \%$ and the maximum below 10\%. The Lagrangian heuristic that we develop for our problem in Chapter 4 is a similar procedure. Using the Lagrangian heuristic, we obtain quite satisfactory results for our batch ordering problem. While implementing the method, (1) we employ an algorithm to solve the subproblems arising as a result of the decomposition in the entire procedure based on using lower and upper bounds on the optimal policy parameters, and (2) we also consider variants of this method that are based on the sequential approach. Consequently, while our problem is more complicated than Wong et al. (2007b) -as we consider $(Q, R)$ policy in the upper echelon-, our heuristics solve yet larger-scale problems with up to 10,000 parts. The other heuristics proposed by Wong et al. (2007b) are tractable for larger problems, but they yield less satisfactory results compared to the DCG, e.g., for the problem instances with 100 parts, the relative gap between the DCG and a lower bound proposed is $0.71 \%$, while for the greedy heuristic which is tractable for large-scale problems, the maximum relative gap is $9.65 \%$.

Caggiano et al. (2007) consider a multi-echelon spare parts inventory distribution system. All facilities operate under a base-stock policy. The demand is Poisson. The objective is to minimize the inventory investments subject to service-level constraints. They consider time-based service levels, e.g., 90\% of parts are instantaneously satisfied, $95 \%$ of the parts are within 8 hours. They propose two alternative heuristics to obtain the base-stock levels at all locations. The first heuristic, FastIncrement, implements a greedy algorithm relying on increasing one of the base-stock levels at a 
time until service level constraints are satisfied. The second heuristic, PrimalDual, resembles our Lagrangian heuristic and that of Wong et al. (2007). In this method the multi-item problem is decomposed into a number of single-item problems using a Lagrangian relaxation. The dual search is accomplished by changing the Lagrangian multipliers until a user-specified limit is reached. Finally this lower bound solution is converted to a feasible integral solution using a greedy algorithm based on incrementing the basetock levels until feasibility is achieved. Both heuristics are shown to be quite efficient and effective. They can solve problems with 27175 part-location combinations in almost 21 hours. The relative gaps with their lower bound are $0.59 \%$ for such problems. Hence, this is one of the papers in the literature having quite high accuracy and efficiency in terms of the computational requirements. As opposed to Caggiano et al. (2007), in our dissertation by using the Lagrangian heuristic, we can solve 130,000 part-location combinations within 12 hours, while the optimality gaps are slightly better than theirs. Although they consider a much more complicated systems than the one in this dissertation, our system is a batch ordering system, which is a generalization of the base-stock systems. Furthermore, in contrast to Caggiano et al. (2007), we consider a column generation method guaranteeing the optimal Lagrangian multipliers to implement the Lagrangian procedure.

There are also papers that deal with the multi-item multi-echelon inventory distribution systems with batch ordering. In an earlier study, Cohen et al. (1990) consider a large spare parts inventory distribution problem for IBM, in which there are millions of part-location combinations. All facilities operate with an $(s, S)$ policy. They also consider the parts commonality among the products. Although their system is a periodic review model, we include in our literature review because of the solution technique is based on the decomposition technique. They develop a multi-item multiechelon model allowing parts commonality to minimize the inventory investments subject to service constraints. They solve this gigantic problem in three stages. In the first stage, the overall problem is reduced to solving multiple single-item singlefacility problems, and then in stage 2 , by integrating these problems a multi-item single-facility problem is solved. In the last stage, the solutions of the problems in stage 2 are embedded into the solution of the overall multi-product multi-location problem. To solve the problem, a level by level decomposition algorithm is used, 
whereas a greedy heuristic is used in order to solve the multi-item single-facility problem.

Among the studies on batch ordering, the papers that are most relevant to our work are Hopp et al. (1999) and Al-Rifai and Rosetti (2007). Hopp et al. (1999) consider a two-echelon spare parts inventory distribution system consisting of a distribution center and multiple nonidentical regional facilities in which the distribution center operates under $(Q, R)$ policy, whereas the regional facilities operate under base-stock policies. The demand is Poisson. The objective is to minimize the inventory holding costs subject to aggregate fill rate and target order frequency constraints. The system differs from ours in two aspects: First, it involves a target level on the aggregate ordering frequency rather than explicit part-specific fixed ordering costs at the upper echelon facility. Second, aggregate fill rate is considered as a service measure, while we consider the aggregate mean response time as a service measure in our work. To find the policy parameters of this system, they decompose the resulting problem by echelons using a Lagrangian relaxation, and then to solve each subproblem, they use the two-moment approximation by Graves (1985) and a sequential heuristic proposed by Hopp et al. (1997), in which the order quantities and the reorder levels are obtained separately. The performance of the heuristic is tested against two alternative lower bounds in a computational study. The results of the experiments reveal that the relative gap between the heuristic solutions and the tighter lower bound is less than $5 \%$. Also, it is experimentally shown that the algorithm can solve a larger problem instance with 1263 parts and 2 regional facilities. Hence, the authors argue that there is a need for further improvements in the algorithm to solve much larger problems encountered in practice.

Al-Rifai and Rosetti (2007) consider a two-echelon spare parts inventory distribution system consisting of one warehouse and multiple identical retailers, all of which operate under $(Q, R)$ policy. The demand is Poisson. The objective is to minimize the average inventory investment subject to average ordering frequency and average backorder constraints. Their system setting differs from ours in three aspects: First, a target level is considered for aggregate ordering frequency rather than fixed ordering costs for each facility. Second, the total expected number of backorders is set as the performance measure rather than the aggregate mean response times. Third, 
they consider only the identical retailer case, whereas our model allows for nonidentical local warehouses. Their heuristic can solve large-scale problems. Similar to Hopp et al. (1999), Al-Rifai and Rossetti (2007) propose a heuristic to find the policy parameters by decomposing the problem by echelons and then applying an iterative heuristic procedure to generate Lagrangian multipliers. Their heuristic relies on the normal approximation of the lead time demand distribution at retailers. Since the order quantities and the reorder levels are determined simultaneously, it can be seen as an application of simultaneous approach. Through a computational study, the performance of the heuristic is tested against the solutions obtained by an optimization software and the results of simulation runs. They show that the heuristic can solve a large-scale problem. Nevertheless, since an analytical solution or a bound is not available for large-scale problems, they encounter difficulties in evaluating the performance of their method in an analytical sense.

In terms of the solution procedure followed, our work in Chapter 4 differs from these two papers in two aspects. (1) In our study, the evaluation of average inventory levels is exact, while Hopp et al. (1999) use a negative binomial approximation, Al-Rifai and Rosetti (2007) use a normal approximation for the lead time demand distribution. (2) The heuristics in both studies, and also ours, rely on the Lagrangian approach, and hence, each one requires a search procedure to obtain the best possible Lagrangian multipliers. In order to find the Lagrangian multipliers, we follow an exact search procedure while the aforementioned studies use iterative heuristic search procedures.

Apart from these papers, there are three review papers on multi-echelon inventory systems. Kennedy et al. (2002) review works on spare parts inventories studying different aspects of spare parts inventory management such as managerial issues, multi-echelon inventory problems, obsolescence, repairable, age-based replacement. Simchi-Levi and Zhao (2010) investigate the methods and the models developed for multi-echelon inventory systems for different network types, inventory polices and demand process. Gümüş and Güneri (2007) survey studies on multi-echelon inventory management published between the years 1996 and 2005 from the perspective of operations research.

This dissertation contributes to the multi-item multi-echelon inventory literature in 
the following three aspects. First, the exact solution procedure that is developed for our system is the first in the literature on multi-item multi-echelon inventory distribution systems. Second, we note that almost none of the algorithms in the literature (except Wong et al. 2007b) guarantee feasible solutions since these algorithms rely on approximate evaluation of the objective function and the constraints. Furthermore, for some of the heuristics, it is not clear whether they are tractable for large, practical size problems (Hopp et al. (1999), Çağlar et al. 2004). The ones that are reported to be tractable for large-scale problems either encounter difficulties in evaluating the performance of their heuristics against an analytical solution or a bound (Al-Rifai and Rossetti 2007), or they are developed for systems under pure basestock policy (Caggiano et al. 2007), Wong et al. 2007b). Hence, our dissertation contributes to the vast literature on multi-item multi-echelon inventory optimization problems by proposing an exact solution method and efficient heuristics based on an exact evaluation -hence guaranteeing feasible solutions- and also a tight lower bound for large-scale practical-size multi-item two-echelon inventory problems with batch ordering at the central warehouse. Third, to the best of our knowledge, our work is the first to propose heuristics for finding optimal or near-optimal policy parameters of a practical industry-size multi-item two-echelon inventory control system with batch ordering facing a compound Poisson demand.

\subsection{Sequential Heuristics}

Another direction of research related to this dissertation is the development of sequential heuristics and investigation of their performances. There are a number of papers studying the sequential heuristic in single-echelon $(Q, R)$ model. Zheng (1992) analyzes the performance of the EOQ with planned backorders formula in a sequential heuristic to obtain the order quantity in a single-item single-echelon $(Q, R)$ model under a general demand distribution. He analytically shows that the EOQ with planned backorders performs well, resulting in a percentage cost penalty of less than $12.50 \%$ theoretically. Through a computational study, he shows that the numerical findings are much better: In $80 \%$ of the numerical problem instances the percentage cost penalty is less than $1.00 \%$, and the maximum percentage cost penalty is found to be 
2.90\%. He also reports that the EOQ with planned backorders performs better when fixed ordering cost is high. Following this line of research, Axsäter (1996), who studies the same problem with Zheng (1992), improves the bound on the percentage cost penalty of using the EOQ with planned backorders down to $11.80 \%$. Silver et al. (1998) focus on the performance of the sequential determination of the order quantities and the reorder levels in single-item single-echelon $(Q, R)$ model and compare its performance against the exact solution obtained by determining $Q$ and $R$ simultaneously. They show that, depending on the problem setting, the percentage cost penalty of using the sequential approach may be high. The authors also suggest making preliminary tests to see whether the simultaneous approach is worthwhile before using it. Gallego (1998) proposes a batch size heuristic that can be used to determine the order quantity in a $(Q, R)$ policy in a sequential approach for a $(Q, R)$ model based on the lower and the upper bounds that he proposes on the order quantity. He assumes a general demand distribution, the analysis is extended to Poisson demand case as well. Both the heuristic and the bounds are reported to perform well. The percentage cost penalty of using the heuristic is shown to be less than $6.07 \%$ theoretically. He empirically shows that the average and the maximum percentage cost penalties of the heuristic are $0.32 \%$ and 2.64 respectively, while those of the EOQ with planned backorders are $1.56 \%$ and 10.61 respectively. In this dissertation, in Section 4.2, we adapt this heuristic to a multi-echelon setting.

In a multi-item setting, Hopp et al. (1997) considers a single-echelon inventory system operating under a $(Q, R)$ policy. The objective is to minimize the inventory investment subject to aggregate fill rate and target order frequency constraints. The demand is Poisson. To obtain the policy parameters they propose three easily implementable heuristics obtained through using closed form expressions. In the first heuristic, the order quantities and the reorder levels are solved separately. The second one requires solving these parameters simultaneously. The third one combines the closed form expressions obtained for the three. Through a computational study they show that the first heuristic, which is based on the sequential approach, may perform poorly but performs better when the target service level is very high and the target order frequency is very low. They report that only in these situation it becomes reasonable to use a sequential approach. 
As seen from the review of papers in Section 2.1, obtaining the order quantities independently or assuming that order quantities are predetermined by using a deterministic model is a very common assumption among the papers on multi-echelon inventory systems (Svoronos and Zipkin 1988, Svoronos and Zipkin 1988, Moinzadeh and Lee 1986, Axsäter 1994, Axsäter 2001, Axsäter 2001, Axsäter 2003, Axsäter 1993, Axsäter 1998, Forsberg 1996, Axsäter 2000). The method is also widely used in practical applications due to its simplicity. However, although the sequential approach is a common approach, its consequences in multi-echelon systems are not investigated yet. In a multi-item multi-echelon setting, Hopp et al. (1999), who consider a sequential heuristic to predetermine the order quantities, can be considered as an application of sequential approach. They test the performance of the heuristic with a lower bound again obtained through sequential approach. Hence, its performance with respect to a simultaneous approach is not considered. In this dissertation, one of our objectives is to evaluate the performance of the widely used sequential approach against the simultaneous approach in multi-item two-echelon batch ordering systems. 


\section{CHAPTER 3}

\section{AN EXACT SOLUTION PROCEDURE}

In this study, a multi-item two-echelon spare parts inventory system is considered. The system consists of a central warehouse and a number of local warehouses, each of which (including the central warehouse) can respond to external customer demand. The central warehouse also responds to the replenishment orders from local warehouses, implying that it has both internal and external demands to satisfy. The central warehouse operates under a continuous-review installation-stock $(Q, R)$ policy and the local warehouses implements a continuous-review installation-stock $(S-1, S)$ policy. The stocks at the central warehouse are replenished from an outside supplier. We assume that the outside supplier has ample stock. Unsatisfied demand is backordered at all locations for each of which an aggregate service level target is set.

Our objective is to find the inventory control policy parameters for this system that will minimize the sum of expected inventory holding and fixed ordering costs subject to constraints on the aggregate mean response time of each facility, which is the demand weighted average of response times. In this chapter, we propose an exact solution procedure based on a branch-and-price algorithm to find the relevant policy parameters of the system considered. The procedure corresponds to solving the Lagrangian dual problem by using a column generation method, and then using this solution as a lower bound in a branch and bound algorithm. The branch-and-price algorithm also involves a greedy algorithm applied on the corresponding Lagrangian dual solution to find an upper bound. Since the column generation method facilitates decomposing the multi-item problem into multiple single-item problems, we also develop an algorithm to solve the single-item problems. 
To the best of our knowledge, there is no exact solution algorithm for finding optimal policy parameters of the multi-item two-echelon inventory problems. Hence, our work contributes to the relevant literature by introducing an exact solution algorithm guaranteeing optimality of the policy parameters for the multi-item two-echelon inventory system that we pose. First, in Section 3.1, we formulate the problem. In Section 3.2, the branch-and-price algorithm is presented. The basic procedures used in the branch-and-price algorithm, i.e., the column generation method, the algorithm developed for solving single-item problems and the greedy algorithm are introduced in Sections 3.2.1, 3.2.2 and 3.2.3, respectively. Finally, in Section 3.4, we provide the computational results.

\subsection{The model}

We consider a two-echelon distribution network in which the lower echelon comprises a set, $N$, of local warehouses, each is denoted by $n=1,2, \ldots|N|$, while the upper echelon corresponds to a central warehouse, which is denoted by $n=0$. There is a set, $I$, of parts, each is denoted by $i=1,2, \ldots|I|$. In this system, we assume that the external customer demand for part $i$ at warehouse $n \in N \cup\{0\}$ occurs according to a Poisson process with rate $\lambda_{i n}$. The external demand is independent across parts and warehouses. In addition to external demands, the central warehouse also faces internal demands from local warehouses. Internal and external demands are not differentiated and are satisfied according to the FCFS rule. For simplicity, we consider a single-indenture model, implying that each part is managed at a product level, but not at the component level. Note that this is validated in many situations (Kim et al. 2009). We assume part-specific holding costs for all facilities and part-specific fixed ordering costs for the central warehouse. There is no incentive for joint ordering of different part types. The demand that can not be satisfied from stock is backordered. Warehouses have no capacity restrictions.

As for the control policies, for each part $i \in I$, local warehouse $n \in N$ operates under a base-stock level $S_{i n}$, whereas the central warehouse operates under a batch ordering policy with reorder level $R_{i}$ and order quantity $Q_{i}$. The system operates as follows: Whenever a demand for any part $i$ arrives at warehouse $n \in N \cup\{0\}$, 
it is immediately satisfied from stocks if there is an available part; otherwise, the demand is backordered. In both cases, if the external demand is directed to a local warehouse, an order of size one is placed at the central warehouse. This internal request is satisfied within a constant transportation lead time of $T_{i n}$, if the part is available in the central warehouse. Otherwise, the internal demand is backordered as well. In any case, if the inventory position of the central warehouse drops to reorder level $R_{i}$, an order of size $Q_{i}$ is placed at the outside supplier. It is assumed that the supplier has ample stock and can always satisfy requests for part $i$ in a constant lead time of $T_{i 0}$. The inventory positions are restricted to be nonnegative, implying that $R_{i} \geq-1$ and $S_{\text {in }} \geq 0$ for each part $i \in I$ and each warehouse $n \in N$. The manufactures considered in our dissertation operate in this manner. This restriction is imposed by other researchers as well (see Hopp et al. 1999, Axsäter 1997). We note that this restriction is not essential for our analysis.

Based on this system definition, our problem can be stated as that of finding policy parameters minimizing the sum of the inventory holding and fixed ordering costs subject to constraints on the demand weighted average of individual part response times over all parts at each warehouse, which we refer to as aggregate mean response time. Our notation is given in Table 3.1.

For sake of brevity, we omit the parameters that the variables depend on (unless there is ambiguity) e.g., $I_{i n}\left(t, Q_{i}, R_{i}, S_{i n}\right)$ is simply denoted as $I_{i n}(t)$. Also, when our focus is on the limiting behavior of a stochastic variable, we omit the time component, e.g., $I_{\text {in }}=\lim _{t \rightarrow \infty} I_{i n}(t)$. Similarly, demands during the respective lead times at the central warehouse and the local warehouse $n \in N$ are shortly denoted by $Y_{i 0}$ and $Y_{i n}$, respectively.

Let $\Lambda_{n}=\sum_{i \in I} \lambda_{i n}$ denote the total demand rate for warehouse $n \in N \cup\{0\}$. By using Little's law, the aggregate mean response time at local warehouse $n \in N, W_{n}(\vec{Q}, \vec{R}, \vec{S})$, can be expressed as a function of expected number of backorders for part $i \in I$, $E\left[B_{\text {in }}\left(Q_{i}, R_{i}, S_{\text {in }}\right)\right]$. 
Table 3.1: General Notation.

\begin{tabular}{|c|c|}
\hline$i$ & Part index, $i \in I$ \\
\hline$n$ & Warehouse index $n \in N \cup\{0\}$ \\
\hline$c_{i}$ & Unit variable cost of part $i$ \\
\hline$h$ & Inventory carrying charge \\
\hline$K_{i}$ & Fixed ordering cost of part $i$ at the central warehouse \\
\hline$\lambda_{\text {in }}$ & Demand rate for part $i$ at local warehouse $n \in N$ \\
\hline$\lambda_{i 0}^{e}$ & External demand rate for part $i$ at the central warehouse \\
\hline$\lambda_{i 0}$ & Demand rate (sum of internal and external) for part $i$ at the central warehouse \\
\hline$\Lambda_{n}^{e}$ & Total external demand rate at the central warehouse \\
\hline$\Lambda_{n}$ & Total demand rate for warehouse $n \in N \cup\{0\}$ \\
\hline$T_{i 0}$ & Lead time for part $i$ at the central warehouse from the outside supplier \\
\hline$T_{\text {in }}$ & Transportation lead time from the central warehouse to local warehouse $n \in N$ for part $i$ \\
\hline$W_{n}^{\max }$ & Target aggregate mean response time at warehouse $n \in N \cup\{0\}$ \\
\hline$R_{i}$ & Reorder level for part $i$ at the central warehouse (decision variable) \\
\hline$Q_{i}$ & Order quantity for part $i$ at the central warehouse (decision variable) \\
\hline$S_{\text {in }}$ & Base-stock level for part $i$ at local warehouse $n \in N$ (decision variable) \\
\hline$\vec{S}_{i}$ & {$\left[S_{i 1}, S_{i 2}, \ldots, S_{i|N|}\right]=$ Vector of base-stock levels for part $i$} \\
\hline$\vec{S}$ & {$\left[\vec{S}_{1}, \vec{S}_{2}, \ldots, \vec{S}_{|I|}\right]=$ Vector of base-stock levels } \\
\hline$\vec{Q}$ & {$\left[Q_{1}, Q_{2}, \ldots, Q_{\mid I]}\right]=$ Vector of order quantities } \\
\hline$\vec{R}$ & {$\left[R_{1}, R_{2}, \ldots, R_{\mid I]}\right]=$ Vector of reorder levels } \\
\hline$I_{i n}\left(t, Q_{i}, R_{i}, S_{i n}\right)$ & On-hand inventory level for part $i$ at warehouse $n \in N$ at time $t$ \\
\hline$I_{i 0}\left(t, Q_{i}, R_{i}\right)$ & On-hand inventory level for part $i$ at the central warehouse at time $t$ \\
\hline$I P_{i 0}\left(t, Q_{i}, R_{i}\right)$ & Inventory position for part $i$ at the central warehouse at time $t$ \\
\hline$X_{i n}\left(t, Q_{i}, R_{i}, S_{i n}\right)$ & Number of outstanding orders for part $i$ at warehouse $n \in N$ at time $t$ \\
\hline$Y_{i n}(t, t+\tau)$ & Demand accumulated for part $i$ at warehouse $n \in N \cup\{0\}$ in time interval $(t, t+\tau)$ \\
\hline$B_{i n}\left(t, Q_{i}, R_{i}, S_{i n}\right)$ & Backorder level for part $i$ at warehouse $n \in N$ at time $t$ \\
\hline$B_{i 0}\left(t, Q_{i}, R_{i}\right)$ & Backorder level for part $i$ at the central warehouse at time $t$ \\
\hline$B_{i 0}^{(n)}\left(t, Q_{i}, R_{i}\right)$ & Backorder level of local warehouse $n$ for part $i$ at the central warehouse at time $t$ \\
\hline$W_{i n}\left(t, Q_{i}, R_{i}, S_{i n}\right)$ & Response time for part $i$ at warehouse $n \in N$ at time $t$ \\
\hline$W_{i 0}\left(t, Q_{i}, R_{i}\right)$ & Response time for part $i$ at the central warehouse at time $t$ \\
\hline$W_{i 0}^{e}\left(t, Q_{i}, R_{i}\right)$ & Response time for part $i$ at the central warehouse (for external customers) at time $t$ \\
\hline$W_{n}(t, \vec{Q}, \vec{R}, \vec{S})$ & Aggregate mean response time at warehouse $n \in N$ at time $t$ \\
\hline$W_{0}(t, \vec{Q}, \vec{R})$ & Aggregate mean response time at the central warehouse at time $t$ \\
\hline$W_{0}^{e}(t, \vec{Q}, \vec{R})$ & Aggregate mean response time at the central warehouse (for external customers) at time $t$ \\
\hline
\end{tabular}




$$
\begin{aligned}
W_{n}(\vec{Q}, \vec{R}, \vec{S}) & =\sum_{i \in I} \frac{\lambda_{i n}}{\Lambda_{n}} E\left[W_{i n}\left(Q_{i}, R_{i}, S_{i n}\right)\right]=\sum_{i \in I} \frac{\lambda_{i n}}{\Lambda_{n}} \frac{E\left[B_{i n}\left(Q_{i}, R_{i}, S_{i n}\right)\right]}{\lambda_{i n}} \\
& =\sum_{i \in I} \frac{E\left[B_{i n}\left(Q_{i}, R_{i}, S_{i n}\right)\right]}{\Lambda_{n}} .
\end{aligned}
$$

Similarly, for the central warehouse, we obtain $W_{0}(\vec{Q}, \vec{R})=\sum_{i \in I} \frac{E\left[B_{i 0}\left(Q_{i}, R_{i}\right)\right]}{\Lambda_{0}}$. Accordingly, the problem $(P)$ is formulated as follows.

$$
\begin{aligned}
& \text { Min } \sum_{i \in I}\left[c_{i} h\left(E\left[I_{i 0}\left(Q_{i}, R_{i}\right)\right]+\sum_{n \in N} E\left[I_{i n}\left(Q_{i}, R_{i}, S_{i n}\right)\right]\right)+\frac{\lambda_{i 0} K_{i}}{Q_{i}}\right] \\
& \text { s.t. } \quad \sum_{i \in I} \frac{E\left[B_{i 0}\left(Q_{i}, R_{i}\right)\right]}{\Lambda_{0}} \leq W_{0}^{\max } \text {, } \\
& \sum_{i \in I} \frac{E\left[B_{i n}\left(Q_{i}, R_{i}, S_{i n}\right)\right]}{\Lambda_{n}} \leq W_{n}^{\max }, \quad \text { for } \forall n \in N, \\
& Q_{i} \geq 1, R_{i} \geq-1, S_{i n} \geq 0, \text { and } Q_{i}, R_{i}, S_{\text {in }} \in Z, \quad \text { for } \forall i \in I, \forall n \in N \text {. }
\end{aligned}
$$

In problem $P$, the objective function (3.1) minimizes the expected system-wide inventory holding and fixed ordering costs. Note that, since we assume full backordering, variable ordering cost are not included in the objective function. Constraint (3.2) and (3.3) guarantee that aggregate mean response times at the central and local warehouses do not exceed target aggregate mean response times, $W_{0}^{\max }$ and $W_{n}^{\max }$, respectively.

Alternatively, one could also model the situation in which only the external customers are incorporated in evaluating the performance of the central warehouse. In that case, the aggregate mean response time at the central warehouse is stated as follows: $W_{0}^{e}(\vec{Q}, \vec{R})=\sum_{i \in I} \frac{\lambda_{i 0}^{e}}{\Lambda_{0}^{e}} E\left[W_{i 0}^{e}\left(Q_{i}, R_{i}\right)\right]$, where $\lambda_{i 0}^{e}=\lambda_{i 0}-\sum_{n \in N} \lambda_{i n}$ is the external demand for part $i \in I$ and $\Lambda_{0}^{e}=\Lambda_{0}-\sum_{n \in N} \Lambda_{n}$ is the total external demand, at the central warehouse. Since there is no differentiation between the external and the internal customers we simply have $W_{0}^{e}(\vec{Q}, \vec{R})=W_{0}(\vec{Q}, \vec{R})$. Then, we obtain

$$
W_{0}^{e}(\vec{Q}, \vec{R})=\sum_{i \in I} \frac{\lambda_{i 0}^{e}}{\Lambda_{0}^{e}} E\left[W_{i 0}\left(Q_{i}, R_{i}\right)\right]=\sum_{i \in I} \frac{\lambda_{i 0}^{e}}{\Lambda_{0}^{e}} \frac{E\left[B_{i 0}\left(Q_{i}, R_{i}\right)\right]}{\lambda_{i 0}}
$$


which replaces constraint (3.2). In a way, this alternative model corresponds to weighing the individual aggregate mean response time values only with the rate of external customers. The rest of this section is devoted to deriving the expected inventory levels in (3.1) and backorder levels in (3.2) and (3.3).

In order to obtain the expected inventory levels and the backorder levels, we use a method similar to the disaggregation method by Axsäter (2006), or so called the leadtime demand method (Simchi-Levi and Zhao 2010). First, since the local warehouses operate under base-stock policies, any demand arrival concurrently triggers an order at the central warehouse, the demand at the central warehouse is the sum of Poisson random variables and its distribution is also Poisson due to superpositioning. Furthermore, since the net inventory for part $i$ at the central warehouse at time $t+T_{i 0}$ is the inventory position at time $t$ minus the demand during lead time $T_{i 0}$, the corresponding inventory balance equation is given by $I_{i 0}\left(t+T_{i 0}\right)-B_{i 0}\left(t+T_{i 0}\right)=I P_{i 0}(t)-Y_{i 0}\left(t, t+T_{i 0}\right)$. Consequently, since $I P_{i 0}$ is uniformly distributed between $R_{i}+1$ and $R_{i}+Q_{i}$ (Axsäter 2006), the steady state distributions of $I_{i 0}$ and $B_{i 0}$ are as follows:

$$
\begin{gathered}
P\left\{I_{i 0}\left(Q_{i}, R_{i}\right)=x\right\}= \begin{cases}\frac{1}{Q_{i}} \sum_{k=\max \left(R_{i}+1, x\right)}^{R_{i}+Q_{i}} P\left\{Y_{i 0}=k-x\right\}, & \text { for } 1 \leq x \leq R_{i}+Q_{i}, \\
\frac{1}{Q_{i}} \sum_{k=R_{i}+1}^{R_{i}+Q_{i}} P\left\{Y_{i 0} \geq k\right\}, & \text { for } x=0,\end{cases} \\
P\left\{B_{i 0}\left(Q_{i}, R_{i}\right)=x\right\}= \begin{cases}\frac{1}{Q_{i}} \sum_{k=R_{i}+1}^{R_{i}+Q_{i}} P\left\{Y_{i 0}=k+x\right\}, & \text { for } x \geq 1, \\
\frac{1}{Q_{i}} \sum_{k=R_{i}+1}^{R_{i}+Q_{i}} P\left\{Y_{i 0} \leq k\right\}, & \text { for } x=0,\end{cases}
\end{gathered}
$$

where $Y_{i 0}$ has a Poisson distribution with mean $\lambda_{i 0} T_{i 0}$. Next, we evaluate the steady state distributions of the inventory levels at local warehouses. Under a base-stock policy, since every demand triggers an order, the inventory position, which is the sum of the net inventory level and the number of outstanding orders, is always constant at the base-stock level. Hence, the inventory position of part $i$ at local warehouse $n$ at time $t$ is

$$
I P_{\text {in }}(t)=S_{\text {in }}=I_{\text {in }}(t)-B_{\text {in }}(t)+X_{\text {in }}(t) .
$$


Furthermore, since for each part $i$, the number of outstanding orders at time $t$ at warehouse $n$ is the number of backorders dedicated to warehouse $n$ at the central warehouse at $t-T_{\text {in }}$ plus the demand during lead time $T_{\text {in }}$ at warehouse $n, X_{i n}(t)$ can be expressed as

$$
X_{i n}(t)=B_{i 0}^{(n)}\left(t-T_{i n}\right)+Y_{i n}\left(t-T_{i n}, t\right) .
$$

Note that $B_{i 0}^{(n)}\left(t, Q_{i}, R_{i}\right)$ can be obtained by conditioning on $B_{i 0}\left(Q_{i}, R_{i}\right)$ as

$$
P\left\{B_{i 0}^{(n)}\left(Q_{i}, R_{i}\right)=x\right\}=\sum_{y=x}^{\infty} P\left\{B_{i 0}^{(n)}\left(Q_{i}, R_{i}\right)=x \mid B_{i 0}\left(Q_{i}, R_{i}\right)=y\right\} \cdot P\left\{B_{i 0}\left(Q_{i}, R_{i}\right)=y\right\}
$$

for $x \geq 0$, where $B_{i 0}^{(n)} \mid B_{i 0}$ is binomially distributed with parameters $B_{i 0}$ and $\frac{\lambda_{i n}}{\lambda_{i 0}}$. In this way, the number of backorders (at the central warehouse) emanating from each local warehouse $n \in N$ is obtained by disaggregation the total number of backorders at the central warehouse. This is known as the binomial disaggregation. It is exact for two-echelon distribution systems with Poisson demand and retailers operating under an $(S-1, S)$ policy (Axsäter 2006), which is the case for system. The binomial disaggregation simplifies the derivations quite a lot. Similarly, by using (3.7), the steady state distribution of $X_{i n}\left(Q_{i}, R_{i}\right)$ can be expressed in terms of the distribution of $B_{i 0}^{(n)}\left(Q_{i}, R_{i}\right)$ as

$$
P\left\{X_{i n}\left(Q_{i}, R_{i}\right)=x\right\}=\sum_{y=0}^{x} P\left\{Y_{i n}=y\right\} \cdot P\left\{B_{i 0}^{(n)}\left(Q_{i}, R_{i}\right)=x-y\right\}, \text { for } x \geq 0,
$$

where $Y_{\text {in }}$ has a Poisson distribution with mean $\lambda_{i n} T_{i n}$. As a result, from (3.6), the steady state distribution of $I_{\text {in }}\left(Q_{i}, R_{i}, S_{\text {in }}\right)$ is

$$
P\left\{I_{\text {in }}\left(Q_{i}, R_{i}, S_{i n}\right)=x\right\}= \begin{cases}P\left\{X_{\text {in }}\left(Q_{i}, R_{i}\right)=S_{\text {in }}-x\right\}, & \text { for } 1 \leq x \leq S_{\text {in }}, \\ 1-\sum_{x=1}^{S_{\text {in }}} P\left\{I_{\text {in }}\left(Q_{i}, R_{i}, S_{\text {in }}\right)=x\right\}, & \text { for } x=0 .\end{cases}
$$


Using the distributions of inventory levels in (3.4) and (3.9), the expected inventory costs in the objective function (3.1) are derived. Finally, the expected backorder expressions in constraints (3.2) and (3.3) are

$$
\begin{aligned}
& E\left[B_{i 0}\right]=E\left[Y_{i 0}\right]-R_{i}-\frac{\left(Q_{i}+1\right)}{2}+E\left[I_{i 0}\right], \\
& E\left[B_{i n}\right]=E\left[X_{i n}\right]-S_{i n}+E\left[I_{i n}\right],
\end{aligned}
$$

which avoids solving a nested set of convolutions.

\subsection{The branch-and-price algorithm}

In this section, an exact solution procedure based on a branch-and-price algorithm is proposed. Branch-and-price is a generalization of the branch and bound algorithm with $L P$-relaxation. A column generation method is used to obtain a lower bound for each subproblem (node) of the branch and bound tree. First, a high-level description of the algorithm is provided, then in Sections 3.2.1-3.2.3, the basic procedures that are used as building blocks of the algorithm are explained.

In the branch-and-price algorithm, at each node of the branch and bound tree, first, by iterating a column generation algorithm we obtain the Lagrangian dual solution of the corresponding node, then, by applying a greedy heuristic on the corresponding Lagrangian dual solution, we find a feasible solution to the original problem $P$. The former solution is used as a lower bound for the corresponding node, and the latter one is used as a candidate for the global upper bound to tighten the bounding scheme and expedite the procedure. Depending on these bounds, a node is either fathomed, or further explored by branching. The procedure is repeated until all nodes are fathomed.

As a lower bound, we consider the Lagrangian dual solution for three reasons. (i) The Lagrangian relaxation of $P$ makes it possible to decompose the multi-item problem into multiple single-item problems. (ii) The Lagrangian dual of our problem does not have the integrality property, i.e., the Lagrangian relaxation of $P$ does not necessarily give an integer solution. As a direct consequence of that, the Lagrangian relaxation has the potential to give a better lower bound than the one that LP-relaxation gives 
(Lübbecke and Desrosiers 2002). (iii) It is known to be a tight lower bound for multiitem two-echelon inventory problems with base-stock control policies (Wong et al. 2007b).

At each iteration of the algorithm, we select the node that provides the lowest average of lower and upper bounds to explore first, because of the superior performance observed in the experiments. As for the branching decision, we consider variable dichotomy, which corresponds to imposing branching constraints on the original variables. That is, any fractional $Q_{i}$ or $R_{i}$ or $S_{\text {in }}$ whose remainder is closest to $1 / 2$ is selected for branching.

An overview of the branch-and-price algorithm is presented in Figure 3.1. In Section 3.2.1, we introduce the column generation method. As discussed in Section 3.2.1, the problem $P$ decomposes by part after implementing the method. In Section 3.2.2, a subroutine is proposed to solve each of these single-item two-echelon subproblems. Finally, in Section 3.2.3, we obtain an upper bound for $P$.

\subsubsection{Obtaining the Lagrangian dual bound for the problem: Column Genera- tion Method}

In this section, first, we introduce the column generation method, based on its implementation on the root node. The additional requirements to implement the algorithm to non-root nodes will be discussed later.

The column generation method is an application of Dantzig-Wolfe decomposition (Guignard 2003), which is widely used in the literature to solve both linear and nonlinear integer programming problems (Barnhart et al. 1998, Lübbecke and Desrosiers 2002). The method relies on an alternative formulation of the original problem $P$, which is known as the master problem (Lübbecke and Desrosiers 2002). The master problem simply corresponds to listing all set of feasible policies for each part $i \in I$ and then selecting exactly one of them. Since the column generation procedure works with the principle of generating only the policies (or as the name suggests columns) that improve the overall solution, it is not necessary to generate all set of columns, instead, one can continue with a restricted set. The method is widely used for solving 


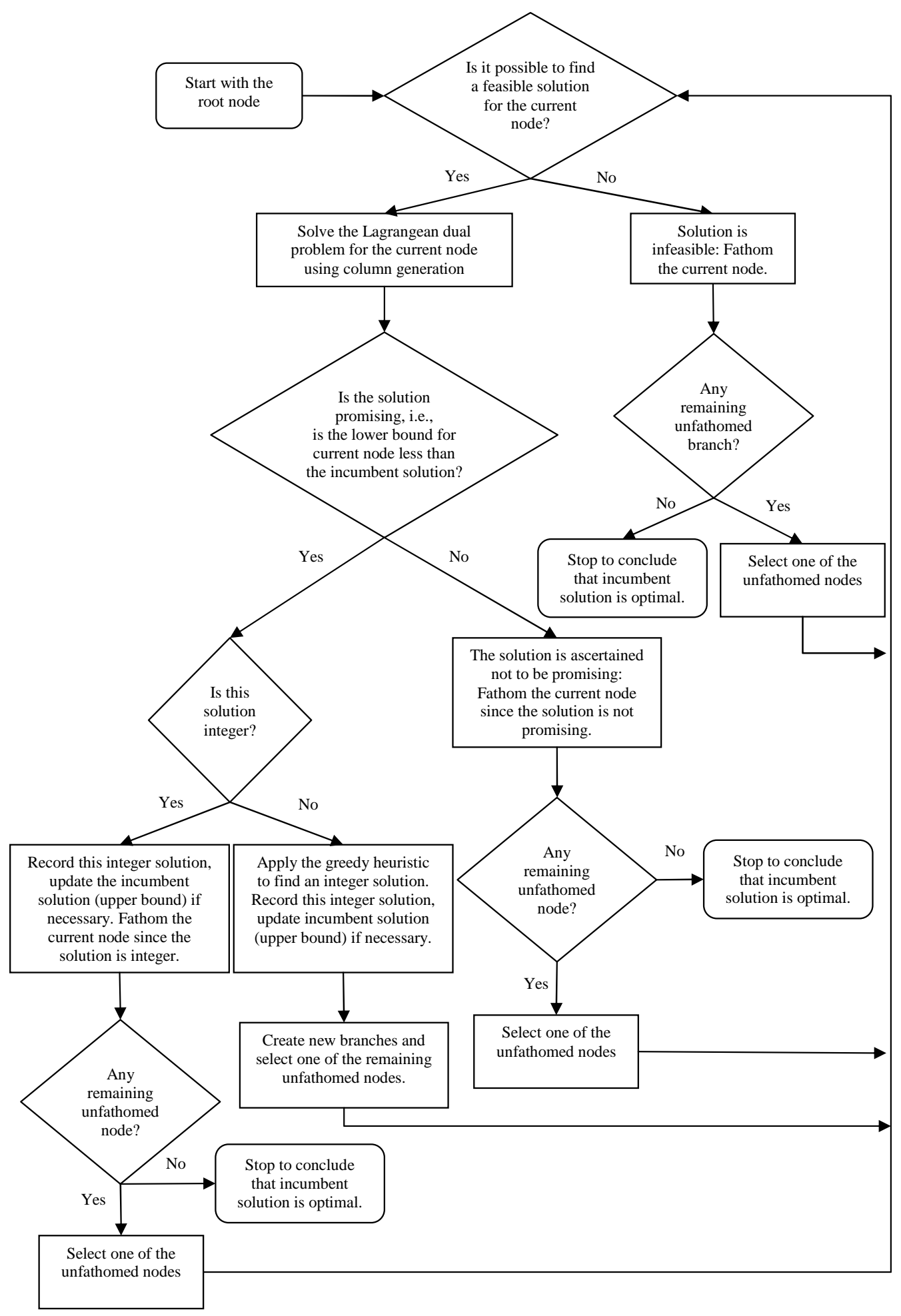

Figure 3.1: The Flowchart of the Branch-and-Price Algorithm. 
various integer programming problems (Lübbecke and Desrosiers 2002, Guignard 2003).

Before giving the details of the algorithm, we first introduce our notation. Let $L$ denote the set of columns, i.e., control policy parameters $\left(Q_{i}, R_{i}, \vec{S}_{i}\right)$, for each part $i$, and $x_{i l}$ be a decision variable indicating whether column $l \in L$ is selected for part $i$ or not. The parameters of our model are defined as follows: Let $C_{i l}=c_{i} h E\left[I_{i 0}\left(Q_{i}^{l}, R_{i}^{l}\right)\right]+$ $c_{i} h \sum_{n \in N} E\left[I_{i n}\left(Q_{i}^{l}, R_{i}^{l}, S_{i n}^{l}\right)\right]+\frac{\lambda_{i 0} K_{i}}{Q_{i}^{l}}$ be the expected total inventory holding and fixed ordering costs associated with column $l \in L$ for part $i$. Similarly let $A_{i l 0}=\frac{E\left[B_{i 0}\left(Q_{i}^{l}, R_{i}^{l}\right)\right]}{\Lambda_{0}}$ and $A_{i l n}=\frac{E\left[B_{i n}\left(Q_{i}^{l}, R_{i}^{l}, S_{i n}^{l}\right)\right]}{\Lambda_{n}}$ be the relevant terms for constraints (3.2) and (3.3) associated with column $l \in L$ for part $i$ for each warehouse $n \in N$, respectively.

The master problem $(M P)$ is formulated as follows:

Problem $M P$ :

$$
\begin{aligned}
& \operatorname{Min} Z=\sum_{i \in I} \sum_{l \in L} C_{i l} x_{i l} \\
& \text { s.t. } \\
& \sum_{i \in I} \sum_{l \in L} A_{i l n} x_{i l} \leq W_{n}^{\max }, \quad \text { for } \forall n \in N \cup\{0\}, \quad\left(\alpha_{n}\right) \\
& \sum_{l \in L} x_{i l}=1, \quad \text { for } \forall i \in I, \quad\left(\beta_{i}\right) \\
& x_{i l}=0 / 1, \quad \text { for } \forall i \in I, \forall l \in L .
\end{aligned}
$$

The problem is known as the Master Problem $(M P)$. It is a tighter formulation than problem $P$, and corresponds to a special case of the set packing problem, which is known to be $\mathcal{N} \mathcal{P}$-hard (Garey and Johnson 1979). The solution of the $L P$-relaxation of problem $M P(L P M P)$ provides a lower bound on the optimal objective function value of $M P$ and hence on that of $P$. This bound corresponds to the Lagrangian dual bound obtained through the Lagrangian relaxation of the constraints of problem $P$ (Guignard 2003). In order to solve problem LPMP, we follow a column generation method by generating only the columns that improve the objective function value of LPMP at each iteration. This restricted version of the problem is known as the Restricted Master Problem (RMP). This step requires solving an integer programming problem known as the column generation $(C G)$ or pricing problem. In light of these, letting $C_{i}\left(Q_{i}, R_{i}, \vec{S}_{i}\right)=c_{i} h E\left[I_{i 0}\left(Q_{i}, R_{i}\right)\right]+c_{i} h \sum_{n \in N} E\left[I_{i n}\left(Q_{i}, R_{i}, S_{i n}\right)\right]+\frac{\lambda_{i 0} K_{i}}{Q_{i}}$, 
$A_{i 0}=\frac{E\left[B_{i 0}\left(Q_{i}, R_{i}\right)\right]}{\Lambda_{0}}$, and $A_{i n}=\frac{E\left[B_{i n}\left(Q_{i}, R_{i}, S_{i n}\right)\right]}{\Lambda_{n}}$ for $i \in I$ and $n \in N$, we introduce the column generation (pricing) problem $(C G)$ as

Problem $C G$ :

$$
\begin{aligned}
& \operatorname{Min} \sum_{i \in I}\left(C_{i}\left(Q_{i}, R_{i}, \vec{S}_{i}\right)-\sum_{n \in N \cup\{0\}} \alpha_{n} A_{i n}-\beta_{i}\right) \\
& \text { s.t. } \\
& Q_{i} \geq 1, R_{i} \geq-1, \vec{S}_{i} \geq \overrightarrow{0} \text {, and } Q_{i}, R_{i}, S_{i n} \in Z, \quad \text { for } \forall i \in I, \forall n \in N,
\end{aligned}
$$

where $\alpha_{n} \leq 0$ for each $n \in N \cup\{0\}$ and $\beta_{i}$ unrestricted in sign for each $i \in I$ are the dual variables (or equivalently Lagrangian multipliers) of problem $M P$, which can be obtained from the solution of $R M P$. In this sense, $C G$ is equivalent to the Lagrangian relaxation of problem $P$ (Barnhart et al. 1998). In an iterative procedure, $C G$ provides the columns that are required for the solution of $L P M P$, whereas $L P M P$ provides the dual variables required for the solution of $C G$.

Although the optimal decisions for different items are linked via constraints (3.2) and (3.3) in $P$, after the Lagrangian relaxation, the corresponding decisions become independent in $C G$, which is an unconstrained problem. Hence, the problem $C G$ is decomposable by parts, and we decompose it into $|I|$ subproblems. Let $\theta_{n}=\frac{-\alpha_{n}}{\Lambda_{n}}$ for each $n \in N \cup\{0\}$ and $\vec{\theta}=\left[\theta_{1}, \theta_{2}, \ldots, \theta_{|N|}\right]$, then the subproblem for part $i \in I$ for a given value of $\vec{\theta}$ is given as follows.

$S P_{i}(\vec{\theta}):$

$$
\begin{array}{ll}
\text { Min } & G\left(Q_{i}, R_{i}, \overrightarrow{S_{i}}\right)=c_{i} h\left(E\left[I_{i 0}\left(Q_{i}, R_{i}\right)\right]+\sum_{n \in N} E\left[I_{i n}\left(Q_{i}, R_{i}, S_{i n}\right)\right]\right)+\frac{\lambda_{i 0} K_{i}}{Q_{i}} \\
+ & \theta_{0} E\left[B_{i 0}\left(Q_{i}, R_{i}\right)\right]+\sum_{n \in N} \theta_{n} E\left[B_{i n}\left(Q_{i}, R_{i}, S_{i n}\right)\right] \\
\text { s.t. } & Q_{i} \geq 1, R_{i} \geq-1, \vec{S}_{i} \geq \overrightarrow{0}, \text { and } Q_{i}, R_{i}, S_{\text {in }} \in Z, \quad \text { for } \forall n \in N .
\end{array}
$$

Let $Z(\vec{\theta})$ and $Z_{i}(\vec{\theta})$ be the optimal objective function values for problems $C G$ and $S P_{i}(\vec{\theta})$, respectively, then $Z(\vec{\theta})$ can be obtained using $Z(\vec{\theta})=\sum_{i \in I}\left(Z_{i}(\vec{\theta})-\beta_{i}\right)$. Further, if at least one of the subproblems $\operatorname{SP}_{i}(\vec{\theta})$ yields a negative optimal objective function value, i.e., $Z_{i}(\vec{\theta})<0$, then the combination of solutions of subproblems with negative objectives is added to set $L$ as a new promising column (solution). Otherwise, optimality is achieved and we conclude that the optimal solution of the $L P$ relaxation of 
$R M P$ becomes optimal for the $L P$ relaxation of $M P$ as well. Note that each time we solve $S P_{i}(\vec{\theta})$, we generate a column for part $i \in I$, i.e., $\left(Q_{i}^{l}, R_{i}^{l}, \vec{S}_{i}^{l}\right)$, that is required for solving $M P$.

The column generation algorithm converges to a solution, provided that a nondegenerate basic feasible solution exists for the master problem (Dantzig 1963). A nondegenerate basic feasible solution for the linear relaxation of $M P$ can be obtained by generating the initial columns that satisfy $\sum_{i \in I} A_{i 0 n} x_{i 0} \leq W_{n}^{\max }$ in the strict sense for each $n \in$ $N$. Hence, the corresponding basic feasible solution consists of the positive slack variables associated with $\sum_{i \in I} \sum_{l \in L} A_{i l n} x_{i l} \leq W_{n}^{\max }$ for each $n \in N$ and the variables $x_{i 0}=1$ for each $i \in I$. We generate the initial solution for the linear relaxation of $M P$ as follows: First, the order quantities are determined by using the EOQ model. Then, using these order quantities, assuming that the target aggregate mean response time at each warehouse $n \in N$, i.e., $W_{n}^{\max }$, should be reached by each part individually, the initial values for the remaining control parameters, i.e., $R_{i}^{0}$ and $S_{i n}^{0}$ for $i \in I$ and $n \in N$, are obtained. This corresponds to obtaining $Q_{i}^{0}, R_{i}^{0}$, and $S_{i n}^{0}$ by using the following formulas in the given order: $Q_{i}^{0}=\left\lfloor E O Q_{i}\right\rfloor, R_{i}^{0}=\operatorname{Min}\left\{R_{i} \in\{-1,0,1, \ldots\}: \frac{E\left[B_{i 0}\left(Q_{i}^{0}, R_{i}\right)\right]}{\lambda_{i 0}} \leq W_{0}^{\max }\right\}$, $S_{i n}^{0}=\operatorname{Min}\left\{S_{\text {in }} \in\{0,1,2, \ldots\}: \frac{E\left[B_{i n}\left(Q_{i}^{0}, R_{i}^{0}, S_{i n}\right)\right]}{\lambda_{\text {in }}} \leq W_{n}^{\max }\right\}$.

Related with the non-root nodes, we have some additional considerations.

- Any column generated by a parent node is introduced also to a child node as long as that column satisfies the branching constraint dedicated to the corresponding child node.

- Although pricing problem $C G$ is an unconstrained problem at the root node, it will involve branching constraints at non-root nodes.

\subsubsection{Solution Procedure for Subproblems: Single-item Two-echelon Batch Or- dering Problem}

To the best of our knowledge, there is no exact solution algorithm proposed for our single-item two-echelon batch ordering problem, $S P_{i}(\vec{\theta})$, although there exist exact procedures for different versions of the problem (Axsäter 1998, Cachon 2001, Moin- 
zadeh and Lee 1986). Therefore, we develop an algorithm to minimize $G\left(Q_{i}, R_{i}, \overrightarrow{S_{i}}\right)$ in (3.14) based on the result that when $Q_{i}$ and $R_{i}$ are fixed it is easy to find the optimal $\vec{S}_{i}$, i.e., $\vec{S}_{i}^{*}\left(Q_{i}, R_{i}\right)$. For this purpose, two nested loops are required; the outer loop searches for the optimal $Q_{i}$, the inner loop searches for the optimal $R_{i}$ for a fixed $Q_{i}$ values, whereas an innermost subroutine optimizes $S_{i n}$ for given values of $Q_{i}$ and $R_{i}$. In Section 3.2.2.1, we derive the optimality conditions for the problem that is solved by the innermost subroutine of this algorithm. In order to reduce the search space, upper bounds $Q_{i}^{U B}$ and $R_{i}^{U B}$, and lower bounds $Q_{i}^{L B}$ and $R_{i}^{L B}$, are proposed for the optimal values for $Q_{i}$ and $R_{i}$, respectively.

In the remainder of this subsection we develop these bounds based on the notions of stochastic domination and supermodularity. The reader is referred to Ross (1996) and Topkis (1998) for further details on stochastic ordering of random variables and supermodularity, respectively.

Lemma 3.2.1 For any $Q_{i}^{+}>Q_{i}$ and $R_{i}^{+}>R_{i}$,
a) $B_{i 0}\left(Q_{i}, R_{i}\right) \geq_{s t} B_{i 0}\left(Q_{i}, R_{i}^{+}\right)$,
b) $B_{i 0}\left(Q_{i}, R_{i}\right) \geq_{s t} B_{i 0}\left(Q_{i}^{+}, R_{i}\right)$,
c) $B_{i 0}^{(n)}\left(Q_{i}, R_{i}\right) \geq_{s t} B_{i 0}^{(n)}\left(Q_{i}, R_{i}^{+}\right)$,
d) $B_{i 0}^{(n)}\left(Q_{i}, R_{i}\right) \geq_{s t} B_{i 0}^{(n)}\left(Q_{i}^{+}, R_{i}\right)$,
e) $X_{\text {in }}\left(Q_{i}, R_{i}\right) \geq_{s t} X_{\text {in }}\left(Q_{i}, R_{i}^{+}\right)$,
f) $X_{\text {in }}\left(Q_{i}, R_{i}\right) \geq_{s t} X_{\text {in }}\left(Q_{i}^{+}, R_{i}\right)$,

where $\geq_{s t}$ denotes stochastic dominance, and $\leq_{s t}$ is defined similarly.

Proof. Proofs of part (a) and (b): By using (3.5), we obtain $P\left\{B_{i 0}\left(Q_{i}, R_{i}\right) \leq x\right\}=$ $\frac{1}{Q_{i}} \sum_{k=R_{i}+1}^{R_{i}+Q_{i}} P\left\{Y_{i 0} \leq k+x\right\}$. This can be used to show $P\left\{B_{i 0}\left(Q_{i}, R_{i}\right) \leq x\right\} \leq P\left\{B_{i 0}\left(Q_{i}, R_{i}^{+}\right) \leq\right.$ $x\}$ and $P\left\{B_{i 0}\left(Q_{i}, R_{i}\right) \leq x\right\} \leq P\left\{B_{i 0}\left(Q_{i}^{+}, R_{i}\right) \leq x\right\}$ for every $x \geq 0$.

Proofs of part (c) and (d): From (3.8), we have

$$
P\left\{B_{i 0}^{(n)}\left(Q_{i}, R_{i}\right)=x\right\}=\sum_{y=x}^{\infty}\left(\begin{array}{c}
y \\
x
\end{array}\right)\left(\frac{\lambda_{i n}}{\lambda_{i 0}}\right)^{x}\left(1-\frac{\lambda_{i n}}{\lambda_{i 0}}\right)^{y-x} P\left(B_{i 0}\left(Q_{i}, R_{i}\right)=y\right) .
$$

From Lemma 3.2.1(a) we have $B_{i 0}\left(Q_{i}, R_{i}\right) \geq_{s t} B_{i 0}\left(Q_{i}, R_{i}^{+}\right)$. Then, $B_{i 0}^{(n)}\left(Q_{i}, R_{i}\right) \geq_{s t}$ 
$B_{i 0}^{(n)}\left(Q_{i}, R_{i}^{+}\right)$as well (see the proof of Lemma 2 (iii) in Moinzadeh and Lee 1986). Part (d) is proved similarly.

Proofs of part (e) and (f): Since $X_{i n}\left(Q_{i}, R_{i}\right)=B_{i 0}^{(n)}\left(Q_{i}, R_{i}\right)+Y_{i n}$, (e) and (f) are direct consequences of (c) and (d), respectively.

Lemma 3.2.2 For any $Q_{i}^{+}>Q_{i}, R_{i}^{+}>R_{i}, S_{i n}^{+}>S_{i n}$, the difference function $\underset{S_{i n}}{\nabla}\left(Q_{i}, R_{i}\right)$ $=I_{\text {in }}\left(Q_{i}, R_{i}, S_{\text {in }}^{+}\right)-I_{\text {in }}\left(Q_{i}, R_{i}, S_{\text {in }}\right)$ satisfies

$$
\begin{aligned}
& \text { a) } \underset{S_{\text {in }}}{\nabla}\left(Q_{i}, R_{i}\right) \leq_{s t} \underset{S_{\text {in }}}{\nabla}\left(Q_{i}, R_{i}^{+}\right) \text {, } \\
& \text { b) } \underset{S_{\text {in }}}{\nabla}\left(Q_{i}, R_{i}\right) \unlhd_{s t} \underset{S_{\text {in }}}{\nabla}\left(Q_{i}^{+}, R_{i}\right) .
\end{aligned}
$$

Proof. Proof of part (a): Since $I_{i n}\left(Q_{i}, R_{i}, S_{\text {in }}\right)=\left(S_{\text {in }}-X_{\text {in }}\left(Q_{i}, R_{i}\right)\right)^{+}$, we have

$$
\underset{S_{\text {in }}}{\nabla}\left(Q_{i}, R_{i}\right)=\left(S_{\text {in }}^{+}-X_{\text {in }}\left(Q_{i}, R_{i}\right)\right)^{+}-\left(S_{\text {in }}-X_{\text {in }}\left(Q_{i}, R_{i}\right)\right)^{+},
$$

where $(\cdot)^{+}=\max (0, \cdot)$. Therefore, we have

$$
\begin{aligned}
& \underset{S_{i n}}{\nabla}\left(Q_{i}, R_{i}\right)= \begin{cases}S_{i n}^{+}-S_{i n}, & \text { for } S_{\text {in }} \geq X_{\text {in }}\left(Q_{i}, R_{i}\right), \\
S_{i n}^{+}-X_{i n}\left(Q_{i}, R_{i}\right), & \text { for } S_{\text {in }}<X_{i n}\left(Q_{i}, R_{i}\right) \leq S_{i n}^{+}, \\
0, & \text { for } S_{\text {in }}^{+}<X_{i n}\left(Q_{i}, R_{i}\right),\end{cases} \\
& P\left\{\underset{S_{i n}}{\nabla}\left(Q_{i}, R_{i}\right)=x\right\}= \begin{cases}P\left\{X_{i n}\left(Q_{i}, R_{i}\right) \geq S_{i n}^{+}\right\}, & \text {for } x=0, \\
P\left\{X_{i n}\left(Q_{i}, R_{i}\right)=S_{i n}^{+}-x\right\}, & \text { for } 0<x<S_{i n}^{+}-S_{i n}, \\
P\left\{X_{i n}\left(Q_{i}, R_{i}\right) \leq S_{i n}^{+}-x\right\}, & \text { for } x=S_{i n}^{+}-S_{i n},\end{cases} \\
& P\left\{\underset{S_{\text {in }}}{\nabla}\left(Q_{i}, R_{i}\right) \leq x\right\}= \begin{cases}P\left\{X_{i n}\left(Q_{i}, R_{i}\right) \geq S_{\text {in }}^{+}\right\}, & \text {for } x=0, \\
P\left\{X_{i n}\left(Q_{i}, R_{i}\right) \geq S_{i n}^{+}-x\right\}, & \text { for } 0<x<S_{i n}^{+}-S_{i n}, \\
1, & \text { for } x \geq S_{i n}^{+}-S_{i n} .\end{cases}
\end{aligned}
$$

Note that for any $x \geq 0$, due to Lemma 3.2.1(e), we have

$$
P\left\{X_{\text {in }}\left(Q_{i}, R_{i}\right) \leq S_{\text {in }}^{+}-x-1\right\} \leq P\left\{X_{\text {in }}\left(Q_{i}, R_{i}^{+}\right) \leq S_{\text {in }}^{+}-x-1\right\},
$$


or equivalently $P\left\{X_{\text {in }}\left(Q_{i}, R_{i}\right) \geq S_{\text {in }}^{+}-x\right\} \geq P\left\{X_{\text {in }}\left(Q_{i}, R_{i}^{+}\right) \geq S_{\text {in }}^{+}-x\right\}$. Combining this result and (3.15), we establish $P\left\{\underset{S_{i n}}{\nabla}\left(Q_{i}, R_{i}\right) \leq x\right\} \geq P\left\{\underset{S_{i n}}{\nabla}\left(Q_{i}, R_{i}^{+}\right) \leq x\right\}$ for any $x \geq 0$.

Proof of part (b): Similarly, by using Lemma 3.2.1(f) and (3.15), we establish $P\left\{\underset{S_{\text {in }}}{\nabla}\left(Q_{i}, R_{i}\right) \leq x\right\} \geq P\left\{\underset{S_{\text {in }}}{\nabla}\left(Q_{i}^{+}, R_{i}\right) \leq x\right\}$.

Corollary 3.2.3 For any $Q_{i}^{+}>Q_{i}, R_{i}^{+}>R_{i}$ and $S_{i n}^{+}>S_{i n}$,

$$
\begin{aligned}
& \text { a) } E\left[I_{\text {in }}\left(Q_{i}, R_{i}, S_{\text {in }}^{+}\right)\right]-E\left[I_{\text {in }}\left(Q_{i}, R_{i}, S_{\text {in }}\right)\right] \leq E\left[I_{\text {in }}\left(Q_{i}, R_{i}^{+}, S_{\text {in }}^{+}\right)\right] \\
& -E\left[I_{\text {in }}\left(Q_{i}, R_{i}^{+}, S_{\text {in }}\right)\right], \text { i.e., } E\left[\underset{S_{\text {in }}}{\nabla}\left(Q_{i}, R_{i}\right)\right] \leq E\left[\underset{S_{\text {in }}}{\nabla}\left(Q_{i}, R_{i}^{+}\right)\right], \\
& \text {b) } E\left[I_{\text {in }}\left(Q_{i}, R_{i}, S_{\text {in }}^{+}\right)\right]-E\left[I_{\text {in }}\left(Q_{i}, R_{i}, S_{\text {in }}\right)\right] \leq E\left[I_{\text {in }}\left(Q_{i}^{+}, R_{i}, S_{\text {in }}^{+}\right)\right] \\
& \text {i.e., }-E\left[I_{\text {in }}\left(Q_{i}^{+}, R_{i}, S_{\text {in }}\right)\right], E\left[\underset{S_{\text {in }}}{\nabla}\left(Q_{i}, R_{i}\right)\right] \leq E\left[\underset{S_{\text {in }}}{\nabla}\left(Q_{i}^{+}, R_{i}\right)\right] .
\end{aligned}
$$

Theorem 3.2.4 $G\left(Q_{i}, R_{i}, \overrightarrow{S_{i}}\right)$ is supermodular in
a) $R_{i}$ and $\overrightarrow{S_{i}}$,
b) $Q_{i}$ and $\overrightarrow{S_{i}}$.

Proof. Proof of part (a): By definition, in order to show that $G\left(Q_{i}, R_{i}, \overrightarrow{S_{i}}\right)$ is supermodular in $R_{i}$ and $\overrightarrow{S_{i}}$, we need to show that for a given value of $Q_{i}, G\left(Q_{i}, R_{i}, \overrightarrow{S_{i}^{+}}\right)-$ $G\left(Q_{i}, R_{i}, \overrightarrow{S_{i}}\right) \leq G\left(Q_{i}, R_{i}^{+}, \overrightarrow{S_{i}^{+}}\right)-G\left(Q_{i}, R_{i}^{+}, \overrightarrow{S_{i}}\right)$ holds for any $\vec{S}_{i}^{+}>\overrightarrow{S_{i}}$ and $R_{i}^{+}>R_{i}$. First, by using the results that $B_{i 0}^{(n)} \mid B_{i 0}$ is binomially distributed with parameters $B_{i 0}$ and $\frac{\lambda_{i n}}{\lambda_{i 0}},(3.7)$ and (3.11), $E\left[B_{i n}\left(S_{i n}, Q_{i}, R_{i}\right)\right]$ or $E\left[I_{i n}\left(S_{i n}, Q_{i}, R_{i}\right)\right]$ can be expressed in terms of the other via

$$
\begin{aligned}
E\left[B_{i n}\left(S_{i n}, Q_{i}, R_{i}\right)\right] & =\frac{\lambda_{i n}}{\lambda_{i 0}} E\left[B_{i 0}\left(Q_{i}, R_{i}\right)\right]+\lambda_{i n} T_{i n}-S_{i n} \\
& +E\left[I_{i n}\left(S_{i n}, Q_{i}, R_{i}\right)\right]
\end{aligned}
$$


Then, substituting (3.16) into the objective function of $S P_{i}(\vec{\theta})$, we obtain

$$
\begin{aligned}
G\left(Q_{i}, R_{i}, \overrightarrow{S_{i}}\right) & =c_{i} h\left(E\left[I_{i 0}\left(Q_{i}, R_{i}\right)\right]+\sum_{n \in N} E\left[I_{i n}\left(Q_{i}, R_{i}, S_{i n}\right)\right]\right) \\
& +\frac{\lambda_{i 0} K_{i}}{Q_{i}}+\theta_{0} E\left[B_{i 0}\left(Q_{i}, R_{i}\right)\right] \\
& +\sum_{n \in N} \theta_{n}\left(\frac{\lambda_{i n}}{\lambda_{i 0}} E\left[B_{i 0}\left(Q_{i}, R_{i}\right)\right]+\lambda_{i n} T_{i n}-S_{i n}+E\left[I_{i n}\left(S_{i n}, Q_{i}, R_{i}\right)\right]\right) \\
& =c_{i} h E\left[I_{i 0}\left(Q_{i}, R_{i}\right)\right]+\frac{\lambda_{i 0} K_{i}}{Q_{i}}+\left(\theta_{0}+\sum_{n \in N} \theta_{n} \frac{\lambda_{i n}}{\lambda_{i 0}}\right) E\left[B_{i 0}\left(Q_{i}, R_{i}\right)\right] \\
& +\sum_{n \in N}\left(c_{i} h+\theta_{n}\right) E\left[I_{i n}\left(Q_{i}, R_{i}, S_{i n}\right)\right]+\sum_{n \in N} \theta_{n}\left(\lambda_{i n} T_{i n}-S_{i n}\right) .
\end{aligned}
$$

Using (3.17), showing $G\left(Q_{i}, R_{i}, \overrightarrow{S_{i}^{+}}\right)-G\left(Q_{i}, R_{i}, \overrightarrow{S_{i}}\right) \leq G\left(Q_{i}, R_{i}^{+}, \overrightarrow{S_{i}^{+}}\right)-G\left(Q_{i}, \overrightarrow{R_{i}^{+}}, \overrightarrow{S_{i}}\right)$ reduces to Corollary 3.2.3(a).

Proof of part (b): Similarly, using (3.17), $G\left(Q_{i}, R_{i}, \overrightarrow{S_{i}^{+}}\right)-G\left(Q_{i}, R_{i}, \overrightarrow{S_{i}}\right) \leq G\left(Q_{i}^{+}, R_{i}, \overrightarrow{S_{i}^{+}}\right)$ $-G\left(Q_{i}^{+}, R_{i}, \overrightarrow{S_{i}}\right)$ reduces to Corollary 3.2.3(b).

Corollary 3.2.5 For a given value of $Q_{i}$, the optimal $R_{i}$ as $S_{\text {in }} \rightarrow \infty$ for each $n \in N$, i.e., $R_{i}^{*}\left(Q_{i}, \lim _{\vec{S}_{i} \rightarrow \vec{\infty}} \vec{S}_{i}\right)$ is a lower bound on the optimal $R_{i}$; the optimal $R_{i}$ for $S_{\text {in }}=0$ for each $n \in N$, i.e., $R_{i}^{*}\left(Q_{i}, \vec{S}_{i}=\overrightarrow{0}\right)$ is an upper bound on the optimal $R_{i}$.

In a similar way, we develop a lower bound on the optimal $Q_{i}$.

Proposition 3.2.6 For any $R_{i}^{+}>R_{i}, Q_{i}^{*}\left(R_{i}, \lim _{\vec{S}_{i} \rightarrow \infty} \vec{S}_{i}\right) \geq Q_{i}^{*}\left(R_{i}^{+}, \lim _{\vec{S}_{i} \rightarrow \infty} \vec{S}_{i}\right)$.

Proof. First, by using (3.16) in (3.14) we establish

$$
\begin{aligned}
G\left(Q_{i}, R_{i}, \vec{S}_{i}\right) & =c_{i} h E\left[I_{i 0}\left(Q_{i}, R_{i}\right)\right]+\frac{\lambda_{i 0} K_{i}}{Q_{i}}+\left(\theta_{0}-c_{i} h \sum_{n \in N} \frac{\lambda_{i n}}{\lambda_{i 0}}\right) E\left[B_{i 0}\left(Q_{i}, R_{i}\right)\right] \\
& +c_{i} h \sum_{n \in N}\left(S_{i n}-\lambda_{i n} T_{i n}\right)+\sum_{n \in N}\left(c_{i} h+\theta_{n}\right) E\left[B_{i n}\left(Q_{i}, R_{i}, S_{i n}\right)\right] .
\end{aligned}
$$

For any given value of $S_{i n}, c_{i} h \sum_{n \in N}\left(S_{i n}-\lambda_{i n} T_{i n}\right)$ is a constant, so it can be excluded from the optimization of (3.18) over $Q_{i}$. Furthermore, as $S_{\text {in }} \rightarrow \infty$ for each $n \in N$, 
$E\left[B_{i n}\left(Q_{i}, R_{i}, S_{i n}\right)\right]=0$. Hence, minimizing (3.18) reduces to minimizing $\bar{G}\left(Q_{i}, R_{i}\right)=$ $c_{i} h E\left[I_{i 0}\left(Q_{i}, R_{i}\right)\right]+\frac{\lambda_{i 0} K_{i}}{Q_{i}}+\left(\theta_{0}-c_{i} h \sum_{n \in N} \frac{\lambda_{i n}}{\lambda_{i 0}}\right) E\left[B_{i 0}\left(Q_{i}, R_{i}\right)\right]$. Note that this function corresponds to the cost function for single echelon $(Q, R)$ policy (see Federgruen and Zheng 1992), for which we have $Q_{i}^{*}\left(R_{i}\right) \geq Q_{i}^{*}\left(R_{i}^{+}\right)$.

Proposition 3.2.7 $Q_{i}^{L B}=Q_{i}^{*}\left(\lim _{R_{i} \rightarrow \infty} R_{i}, \lim _{\vec{S}_{i} \rightarrow \infty} \vec{S}_{i}\right)$ is a lower bound on the optimal $Q_{i}$.

Proof. As a direct consequence of Theorem 3.2.4(b), for any $R_{i}$ we have $Q_{i}^{*}\left(R_{i}, \lim _{\vec{S}_{i} \rightarrow \vec{\infty}} \vec{S}_{i}\right)$ $\leq Q_{i}^{*}\left(R_{i}, \vec{S}_{i}\right)$. Due to Proposition 3.2.6, $Q_{i}^{*}\left(\lim _{R_{i} \rightarrow \infty} R_{i}, \lim _{\vec{S}_{i} \rightarrow \infty} \vec{S}_{i}\right) \leq Q_{i}^{*}\left(R_{i}, \lim _{\vec{S}_{i} \rightarrow \infty} \vec{S}_{i}\right)$. Hence, $Q_{i}^{*}\left(\lim _{R_{i} \rightarrow \infty} R_{i}, \lim _{\vec{S}_{i} \rightarrow \infty} \vec{S}_{i}\right) \leq Q_{i}^{*}\left(R_{i}, \vec{S}_{i}\right)$ holds for any $R_{i}$ and $\vec{S}_{i}$.

Finally, to obtain an upper bound on the optimal $Q_{i}$ we utilize the upper bound on the optimal order quantities in single echelon $(Q, R)$ policies (Gallego 1998).

Proposition 3.2.8 $Q_{i}^{U B}=\sqrt{\left(2 K_{i} \lambda_{i 0}+\left(c_{i} h+p_{i}\right) \lambda_{i 0} T_{i 0}\right) / H_{i}}$, where $H_{i}=\frac{c_{i} h p_{i}}{c_{i} h+p_{i}}$, and $p_{i}=\theta_{0}+\sum_{n \in N} \theta_{n} \frac{\lambda_{i n}}{\lambda_{i 0}}$, is an upper bound on the optimal $Q_{i}$.

Proof. In order to show that $Q_{i}^{U B}$ is an upper bound on the optimal $Q_{i}$, it is sufficient to show that for any $Q_{i}>Q_{i}^{U B}, \overrightarrow{S_{i}}$ and $R_{i}, G\left(Q_{i}, R_{i}, \overrightarrow{S_{i}}\right)>G\left(Q_{i}^{-}, R_{i}, \overrightarrow{S_{i}}\right)$ holds, where $G\left(Q_{i}, R_{i}, \overrightarrow{S_{i}}\right)$ is given in (3.17) and $Q_{i}^{-}<Q_{i}$. Let $C\left(Q_{i}, R_{i}\right)=c_{i} h E\left[I_{i 0}\left(Q_{i}, R_{i}\right)\right]+$ $\frac{\lambda_{i 0} K_{i}}{Q_{i}}+\left(\theta_{0}+\sum_{n \in N} \theta_{n} \frac{\lambda_{i n}}{\lambda_{i 0}}\right) E\left[B_{i 0}\left(Q_{i}, R_{i}\right)\right]$. Then, $C\left(Q_{i}, R_{i}\right)$ corresponds to the cost function in Gallego (1998) where it is shown that $Q_{i}^{U B}$ is an upper bound on the optimal $Q_{i}$ for $C\left(Q_{i}, R_{i}\right)$. Furthermore, since $C\left(Q_{i}, R_{i}\right)$ is unimodal with respect to $Q_{i}$ (Federgruen and Zheng 1992), for any $Q_{i}>Q_{i}^{U B}$ we have $C\left(Q_{i}, R_{i}\right)>C\left(Q_{i}^{-}, R_{i}\right)$. Finally, using this result, $E\left[I_{i n}\left(Q_{i}, R_{i}, S_{i n}\right)\right] \geq E\left[I_{\text {in }}\left(Q_{i}^{-}, R_{i}, S_{i n}\right)\right]$, and that $\sum_{n \in N} \theta_{n}\left(\lambda_{i n} T_{i n}-S_{i n}\right)$ is constant with respect to $Q_{i}$, we establish $G\left(Q_{i}, R_{i}, \overrightarrow{S_{i}}\right)>G\left(Q_{i}^{-}, R_{i}, \vec{S}_{i}\right)$.

\subsubsection{Finding Optimal Solution for Subproblems for Given Values of Reorder Level and Order Quantity}

For a given part $i \in I$, and given values of $Q_{i}$ and $R_{i}, S P_{i}(\vec{\theta})$ reduces to $|N|$ independent subproblems, each of which is denoted by $S P_{i n}\left(\theta_{n}, Q_{i}, R_{i}\right)$. 


$$
\begin{aligned}
& \text { Min } c_{i} h E\left[I_{i n}\left(Q_{i}, R_{i}, S_{i n}\right)\right]+\theta_{n} E\left[B_{i n}\left(Q_{i}, R_{i}, S_{i n}\right)\right] \\
& \text { s.t. } S_{\text {in }} \geq 0 \text {, and } \in Z .
\end{aligned}
$$

By using equation (3.11), the objective function in $S P_{i n}\left(\theta_{n}, Q_{i}, R_{i}\right)$ can be restated as

$$
G\left(S_{i n}\right)=\left(c_{i} h+\theta_{n}\right) E\left[I_{i n}\left(Q_{i}, R_{i}, S_{i n}\right)\right]+\theta_{n} E\left[X_{i n}\left(Q_{i}, R_{i}\right)\right]-\theta_{n} S_{i n}
$$

Proposition 3.2.9 $G\left(S_{\text {in }}\right)$ is unimodal.

Proof. Let $\Delta_{x}$ and $\Delta_{x}^{2}$ be the first and second order difference equations with respect to variable $x$, respectively. Then, $\Delta_{S_{i n}}^{2} G\left(S_{i n}\right) \geq 0$, is a sufficient condition for $G\left(S_{i n}\right)$ to be unimodal. First, from (3.9) we have

$$
E\left[I_{i n}\left(Q_{i}, R_{i}, S_{i n}\right)\right]=\sum_{x=0}^{S_{\text {in }}-1}\left(S_{\text {in }}-x\right) \cdot P\left\{X_{i n}\left(Q_{i}, R_{i}\right)=x\right\}
$$

Using this result, next, we have

$$
\begin{gathered}
\underset{S_{\text {in }}}{\Delta G}\left(S_{i n}\right)=\left(c_{i} h+\theta_{n}\right) \sum_{x=0}^{S_{i n}} P\left\{X_{i n}\left(Q_{i}, R_{i}\right)=x\right\}-\theta_{n}, \\
\underset{S_{\text {in }}}{\Delta} G\left(S_{\text {in }}\right)=\left(c_{i} h+\theta_{n}\right) P\left\{X_{i n}\left(Q_{i}, R_{i}\right)=S_{\text {in }}+1\right\},
\end{gathered}
$$

which satisfies $\underset{S_{\text {in }}}{\Delta^{2}} G\left(S_{i n}\right) \geq 0$.

Proposition 3.2.10 The optimal solution of $S P_{i n}\left(\theta_{n}, Q_{i}, R_{i}\right)$ is

$$
\operatorname{Min}_{S_{i n} \in\{0,1,2, \ldots\}}\left\{S_{\text {in }}: \sum_{x=0}^{S_{\text {in }}} P\left(X_{\text {in }}\left(Q_{i}, R_{i}\right)=x\right) \geq \frac{\theta_{n}}{c_{i} h+\theta_{n}}\right\} .
$$

Proof. As a direct consequence of Proposition 3.2.9, the optimal $S_{\text {in }}$ is the smallest integer that satisfies the first order condition, i.e., ${ }_{S_{i n}} G\left(S_{i n}\right) \geq 0$. 


\subsubsection{Generating Upper Bounds: Greedy Algorithm}

We obtain an upper bound for each node of the branch and bound tree using a greedy heuristic. The greedy algorithm is a simple search algorithm that can be used to generate a feasible solution from an integer but infeasible (dual) solution. The method is known to perform quite well in multi-item two-echelon inventory control problems (Wong et al. 2005, 2006, 2007a, 2007b). The main idea of the greedy algorithm is as follows: Starting with an infeasible solution, at each iteration, the algorithm iterates to a solution that is as close to the feasible region as possible while incurring as low additional cost as possible. This procedure is repeated until a feasible solution is obtained. Since the initial dual solution may yield fractional variables, this may require rounding fractional variables down to make sure that the new solution satisfies constraints (3.2) and (3.3) before iterating the greedy algorithm.

Recall that $\vec{Q}, \vec{R}$, and $\vec{S}$ are vectors of order quantities, reorder levels, and base-stock levels, respectively. Then, one can define the maximum constraint violation for given values of $\vec{Q}, \vec{R}$, and $\vec{S}$ as

$$
\omega(\vec{Q}, \vec{R}, \vec{S})=\max _{n \in N \cup\{0\}}\left\{\left(W_{n}(\vec{Q}, \vec{R}, \vec{S})-W_{n}^{\max }\right)^{+}\right\} .
$$

Also, let $Z(\vec{Q}, \vec{R}, \vec{S})$ be the value of objective function (3.1) for given values of $\vec{Q}, \vec{R}$, and $\vec{S}$. Then, the neighborhood of $(\vec{Q}, \vec{R}, \vec{S}), V(\vec{Q}, \vec{R}, \vec{S})$, is defined as the set of all vectors $[\vec{Q}, \vec{R}, \vec{S}]+\varepsilon$, where $\varepsilon$ is a vector in which exactly one of the entries is one and the rest are zero. Then, the greedy algorithm searches for the solution $\left(\vec{Q}^{\prime}, \vec{R}^{\prime}, \overrightarrow{S^{\prime}}\right) \in$ $V(\vec{Q}, \vec{R}, \vec{S})$ that yields the maximum $r\left(\vec{Q}^{\prime}, \vec{R}^{\prime}, \vec{S}^{\prime}\right)=\frac{\omega\left(\vec{Q}^{\prime}, \vec{R}^{\prime}, \vec{S}^{\prime}\right)-\omega(\vec{Q}, \vec{R}, \vec{S})}{Z\left(\vec{Q}^{\prime}, \vec{R}^{\prime}, \vec{S}^{\prime}\right)-Z(\vec{Q}, \vec{R}, \vec{S})}$ ratio. The greedy algorithm converges finitely by nature.

In our study we use the optimal solution of the LP relaxation of the MP as a starting dual solution for the greedy algorithm, and this often yields fractional solutions. However, the greedy algorithm can only work with integer solutions. To fix this problem, we round down the fractional decision variables.

The greedy algorithm always finds a feasible solution in a finite number of steps. Also, as shown in Section 3.2.1, the column generation algorithm guarantees conver- 
gence. These two show that the overall procedure guarantees convergence.

In this dissertation, we use the greedy algorithm for two more reasons: (1) To solve the problem $P$ heuristically by combining it with the column generation method introduced, which we call the overall procedure as the Lagrangian heuristic, (2) To obtain alternative heuristics for $P$ by integrating it with the sequential heuristics. How it is used in the development of the heuristics are to be discussed in Chapter 4.

\subsection{Computational Results}

To provide insight into the size of problems that can be solved by the exact algorithm, we provide some computational results. We randomly generate a testbed based on the available data obtained from the practical applications mentioned in Chapter 1. We consider problems with 5-30 parts and 2-4 local warehouses. For each pair of these parameters, 10 random instances are generated, where the demand rate, the fixed ordering cost, the unit variable cost and the lead time are randomly generated, while other parameters are not varied as shown in Table 3.2. The demand rates for each part $i$ is generated from a uniform distribution $U[0.01,0.05]$. Further, by multiplying this random number with another uniform random number generated from $U[0.5,1.5]$, we obtain part-specific location-dependent demand rates, i.e., $\lambda_{i n}$, for each part $i$ and warehouse $n \in N \cup\{0\}$. The algorithm is coded in $\mathrm{C}++$ and the experiment is run on an Intel $3 \mathrm{GHz}$ processor with $3.5 \mathrm{~GB}$ RAM. The results of the experiment are presented in Table 3.3. As can be seen in Table 3.2, the exact solution procedure that we propose can be used in applications as long as the number of items and the number of warehouses are limited: as the number of parts and especially the number of warehouses increase, the solution requires significant computational effort. Hence, one may need to apply heuristic approaches for the solution of larger problems.

\subsection{Conclusion}

In this chapter, we propose a branch-and-price algorithm to find the relevant policy parameters of a multi-item two-echelon inventory distribution system in which the 
Table 3.2: Parameter values for the testbed.

\begin{tabular}{|l|c|}
\hline \multicolumn{1}{|c|}{ Parameters } & Values \\
\hline$\lambda_{\text {in }}$ (units/days) & $\mathrm{U}[0.01,0.05] \mathrm{X} \mathrm{U}[0.5,1.5]$ \\
$c_{i}$ (\$/unit) & $\mathrm{U}[1000,5000]$ \\
$K_{i}$ (\$/order) & $\mathrm{U}[50,150]$ \\
$T_{\text {in }}$ (days) & $\mathrm{U}[0.5,1.5]$ \\
$h$ (per year) & 0.25 \\
$T_{i 0}$ (days) & 5 \\
$W_{0}^{\max }$ (days) & 1 \\
$W_{n}^{\max }$ (days) & 0.2 \\
\hline
\end{tabular}

Table 3.3: CPU times for different problem sizes.

\begin{tabular}{|c|c|c|}
\hline $\begin{array}{c}\text { Number of } \\
\text { parts }\end{array}$ & $\begin{array}{c}\text { Number of } \\
\text { local } \\
\text { warehouses }\end{array}$ & $\begin{array}{c}\text { Average } \\
\text { CPU time } \\
\text { (sec) }\end{array}$ \\
\hline 5 & 2 & 1.05 \\
10 & 2 & 15.59 \\
15 & 2 & 182.77 \\
20 & 2 & 208.43 \\
25 & 2 & 788.24 \\
30 & 2 & 6184.72 \\
\hline 5 & 3 & 2.02 \\
10 & 3 & 88.69 \\
15 & 3 & 773.84 \\
20 & 3 & 8175.12 \\
25 & 3 & 17859.96 \\
\hline 5 & 4 & 4.42 \\
10 & 4 & 362.66 \\
15 & 4 & 3298.28 \\
20 & 4 & 20392.54 \\
\hline
\end{tabular}


central warehouse operates under a $(Q, R)$ policy, and each local warehouse implements an $(\mathrm{S}-1, \mathrm{~S})$ policy. The procedure involves a column generation algorithm to find the Lagrangian dual bound and a greedy algorithm to convert this solution to a feasible solution. Using the Lagrangian dual bound as a lower bound and the feasible solution as an upper bound in the solution of each node of the branch-and-bound tree, we develop the branch-and-price algorithm. We experimentally show that the branchand-price algorithm can be used in applications as long as the number of items and the number of warehouses are limited. Even if this is the case, an exact solution is desirable due to significant cost reductions and benchmark purposes. For the solution of large practical problems, one may need to apply heuristic approaches. For this purpose, one can use the column generation method and the greedy algorithm as building blocks and develop heuristics for the problem, which is the subject of the next chapter. 


\section{CHAPTER 4}

\section{HEURISTIC PROCEDURES}

In this chapter, we propose four alternative heuristics to find the optimal or nearoptimal policy parameters of the multi-item two-echelon inventory distribution system considered in Chapter 3. Our emphasize is on large industry-size problems. First, we develop a Lagrangian heuristic employing the column generation method and the greedy algorithm introduced in Chapter 3. We also consider three variants of this heuristic, which are based on the sequential determination of policy parameters, as done frequently in practice. That is, first the order quantities are determined using a batch size heuristic, then the reorder levels at the central warehouse and the basestock levels at the local warehouses are determined through the same method used for the Lagrangian heuristic. As opposed to the heuristics for multi-echelon inventory optimization problems in the literature, our heuristics guarantee feasibility. In an extensive computational study, we test the performances of the heuristics. Since the exact solution is intractable for practical size problems, we consider the Lagrangian dual bound that we develop in Section 3.2.1 as a benchmark solution in the experiments. Therefore, we also investigate performance of the Lagrangian dual bound both theoretically and empirically. We show that this bound is asymptotically tight in the number of parts.

First, the Lagrangian heuristic and the sequential heuristics are introduced in Sections 4.1 and 4.2, respectively. Then, we study the asymptotic analysis of the Lagrangian dual bound in Section 4.3. In Section 4.4, we present and discuss the results of the computational study through which we evaluate the performance of the heuristics and the Lagrangian dual bound. Finally, in Section 4.5 we draw the conclusions. 


\subsection{Lagrangian Heuristic}

"Lagrangian heuristic" is a generic name given to heuristics that first employ a Lagrangian relaxation to find a good -but often infeasible- relaxed solution, and then an algorithm to transform this relaxed solution into a feasible solution (Guignard 2003). In our dissertation, the Lagrangian heuristic simply corresponds to the entire procedure in which the column generation and the greedy algorithms introduced in Chapter 3 are integrated. By using the column generation algorithm in Section 3.2.1, we obtain the Lagrangian dual solution for problem $P$. Since this solution is quite often infeasible, we use the greedy algorithm in Section 3.2.3 to convert this solution to a feasible solution. In this way, we obtain the Lagrangian heuristic. Note that this solution procedure corresponds to the upper bounding procedure used in the branch-and-price algorithm. Therefore, the solution itself corresponds to the upper bound obtained for the root node of the branch-and-price algorithm for the problem. Similarly, the Lagrangian dual bound corresponds to the lower bound obtained for the root node of the branch-and-price algorithm for the problem. This explains the basic relationship between our branch-and-price algorithm, Lagrangian heuristic and Lagrangian dual bound. Note that since the Lagrangian heuristic is based on determining the order quantities and the reorder levels simultaneously, it is a simultaneous approach heuristic. An overview of the Lagrangian heuristic is given in Figure 4.1.

\subsection{Sequential Heuristics}

Similar to the Lagrangian heuristic, the sequential heuristics rely on the integration of the column generation and the greedy algorithm. However, in contrast to the Lagrangian heuristic, the order quantities at the central warehouse are determined offline. The sequential heuristics iterate as follows: First, the order quantities are determined through a batch size heuristic. Then, given the order quantities, the remaining policy parameters, i.e., the reorder levels at the central warehouse and the base-stock levels at the local warehouses, are determined by using the entire procedure developed for the Lagrangian heuristic. This results in changes in the overall procedure: In the column generation algorithm in Section 3.2.1, $Q_{i}$ is discarded from problem 


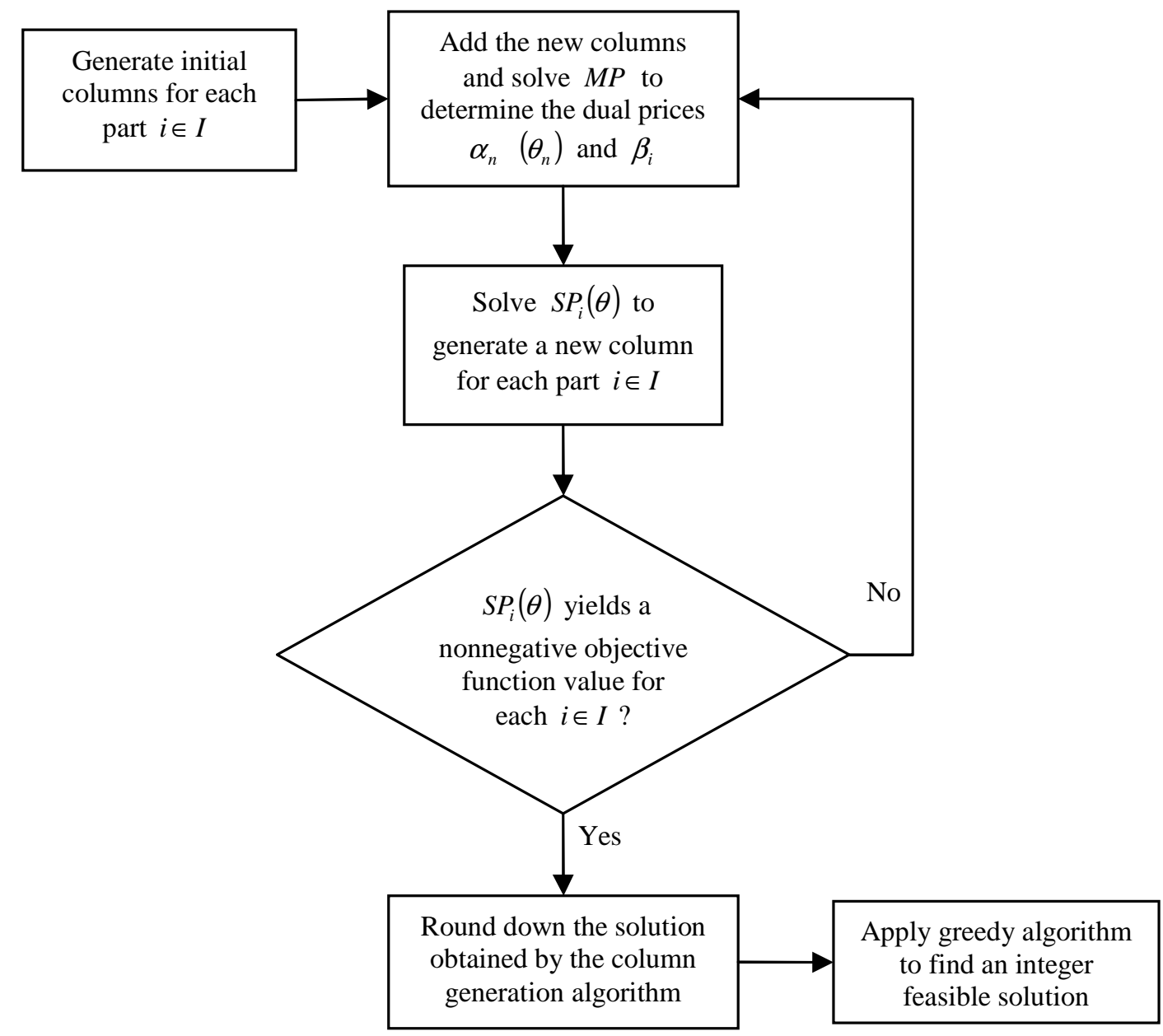

Figure 4.1: The Flowchart of the Lagrangian Heuristic. 
$S P_{i}(\vec{\theta})$ for each $i \in I$, hence the outer loop of the algorithm proposed to solve $S P_{i}(\vec{\theta})$ is eliminated. This also brings a computational advantage to the sequential heuristics over the Lagrangian heuristic. An overview of the sequential heuristics is given in Figure 4.2.

To implement the sequential approach, we consider three alternatives for setting the order quantities:

- the EOQ formula, i.e., $Q_{i}=\sqrt{\frac{2 \lambda_{i 0} K_{i}}{c_{i} h}}$,

- the EOQ with planned backorders $\left(E O Q^{B}\right)$ formula, i.e., $Q_{i}=\sqrt{\frac{2 \lambda_{i 0} K_{i}\left(c_{i} h+p_{i}\right)}{\left(c_{i} h\right) p_{i}}}$ (Zheng 1992, Gallego 1998), where $p_{i}$ is the shortage cost defined per unit short of part $i \in I$ per unit time and obtained as it is described in Proposition 3.2.8 in Section 3.2.2,

- an alternative batch size heuristic $Q^{L U}$ based on the lower and upper bounds, $Q_{i}^{L B}$ and $Q_{i}^{U B}$, that we obtain for the single-item two-echelon batch ordering problem $S P_{i}(\vec{\theta})$ in Propositions 3.2.7 and 3.2.8, respectively in Section 3.2.2. The heuristic is similar to the batch size heuristic proposed by Gallego (1998) for the single-echelon $(Q, R)$ model. However, when Gallego's batch size heuristic is directly used in our model, i.e., $Q_{i}=\min \left(\sqrt{2} Q_{i}^{L B}, \sqrt{Q_{i}^{L B} \cdot Q_{i}^{U B}}\right)$, the optimal order quantities are overestimated. Hence, we adopt it in our model by using the harmonic mean of $Q_{i}^{L B}$ and $Q_{i}^{U B}$ instead of using a geometric mean, which is less than or equal to the latter. In this way, we achieve better results. Accordingly, the order quantities are found from $Q_{i}=\min \left(\sqrt{2} Q_{i}^{L B}, \frac{2 Q_{i}^{L B} Q_{i}^{U B}}{Q_{i}^{L B}+Q_{i}^{U B}}\right)$.

In this manner, we obtain three alternative sequential heuristics, $S_{1}, S_{2}$ and $S_{3}$, respectively.

The batch size heuristics differ depending on how the service level requirements are taken into account in determining the order quantities. In $S_{1}$, the order quantities are determined independent of the service level requirements. This is the case in many practical applications, e.g., the manufacturers considered in our work determine the order quantities using the EOQ. However, $S_{2}$ and $S_{3}$ incorporate the service level requirements by means of a part-specific shortage cost, $p_{i}$, for each part $i \in I$. In 


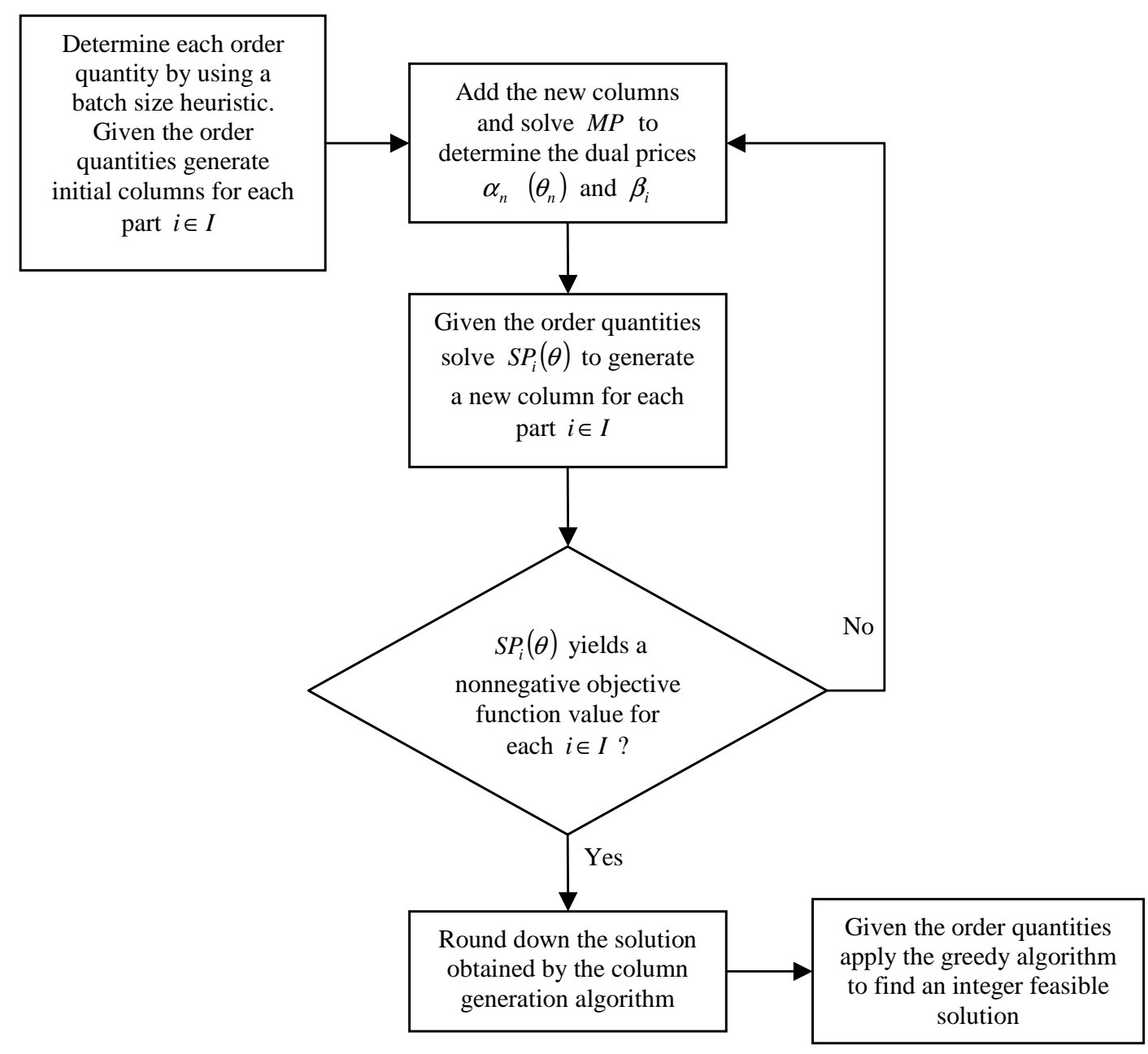

Figure 4.2: The Flowchart of the Sequential Heuristics. 
order to obtain each part-specific shortage cost, $p_{i}$, first, we apply the entire procedure in Figure 4.2 by using the EOQ, and then under the solution obtained, we compute the probability of no stockouts, $\gamma_{i}$, for each part $i \in I$. Then, by substituting $\gamma_{i}$ in the newsboy ratio $\gamma_{i}=\frac{p_{i}}{c_{i} h+p_{i}}$, we determine $p_{i}$. Finally, the entire procedure iterates once more to obtain the solution of the corresponding sequential heuristic. Therefore, while the corresponding procedure iterates once in $S_{1}$, it iterates twice in $S_{2}$ and $S_{3}$; first to find the part part-specific shortage costs, second to obtain the overall solution. Since the greedy algorithm converges finitely and the column generation algorithm is guaranteed to converge to a solution, all our heuristics guarantee convergence. The heuristics proposed in this chapter are summarized in Table 4.1.

Table 4.1: Heuristics proposed.

\begin{tabular}{|l|l|}
\hline \multicolumn{1}{|c|}{ Solution Approach } & \multicolumn{1}{|c|}{ Heuristics } \\
\hline $\begin{array}{l}\text { Simultaneous Approach } \\
\text { - All the control parameters are } \\
\text { determined simultaneously. }\end{array}$ & $\begin{array}{l}\text { - The order quantities and reorder points at the } \\
\text { central warehouse, basestock levels at local } \\
\text { warehouses are obtained by using the column } \\
\text { generation and the greedy algorithms. }\end{array}$ \\
\hline $\begin{array}{l}\text { Sequential Approach } \\
\text { Order quantities are predetermined } \\
\text { by using a batch size heuristic. }\end{array}$ & $\begin{array}{l}\text { Given order quantities, reorder points at the } \\
\text { central warehouse, and basestock levels at } \\
\text { local warehouses are obtained by using the } \\
\text { column generation and the greedy algorithms. } \\
\circ \mathrm{EOQ}^{\mathrm{B}}\end{array}$ \\
$\mathrm{O} \mathrm{Q}^{\mathrm{LU}}$ & $\circ \mathrm{S}_{1}$ (uses EOQ) \\
$\mathrm{S}_{2}$ (uses EOQ & $\circ \mathrm{S}_{3}$ (uses $\left.\mathrm{Q}^{\mathrm{LU}}\right)$ \\
\hline
\end{tabular}

\subsection{Asymptotic analysis of the Lagrangian dual bound}

In this chapter, we use the Lagrangian dual bound to test the performance of the heuristics for practical size problems. Before that, we first analyze the performance of the Lagrangian dual bound. In this section, we study the asymptotic behaviour of the Lagrangian dual bound for our problem and show that the Lagrangian dual bound is asymptotically tight in the number of parts. The analysis relies on the probabilistic analysis of combinatorial problems (Kellerer et al. 2004). Accordingly, we 
assume that for each $i \in I$ and $n \in N \cup\{0\}, c_{i}, K_{i}, \lambda_{i n}$ and $W_{n}^{\max }$ are independent and identically distributed random variables drawn from a uniform distribution $U[\underline{c}, \bar{c}]$, $U[\underline{K}, \bar{K}], U[\underline{\lambda}, \bar{\lambda}]$ and $U[\underline{W}, \bar{W}]$, respectively. We further assume that $\underline{c}, \underline{W}>0$ and $\bar{K}, \bar{c}, \bar{\lambda}<\infty$, implying that

- the fixed ordering cost is strictly finite for each part $i \in I$, i.e., $K_{i}<\infty$,

- the unit holding cost is strictly positive and finite for each part $i \in I$, i.e., $0<$ $c_{i} h<\infty$,

- the target aggregate mean response time at each warehouse $n \in N \cup\{0\}$ is strictly positive, i.e., $W_{n}^{\max }>0$,

- the average lead time demand for each part $i \in I$ at each warehouse $n \in N \cup\{0\}$ is finite, i.e., $\lambda_{i n} T_{i n}<\infty$.

Note that these four assumptions are practically nonrestrictive, but necessary for our model to be stable and the problems to have finite solutions.

Through Theorem 4.3.2, we first show that the optimal objective function value of $M P, z^{M P}$, increases at least linearly with the number of parts. Then, in Theorem 4.3.5, we show that the gap between the optimal objective function value of $M P, z^{M P}$, and its $L P$-relaxation, $z^{L P M P}$, grows only with an order of the number of local warehouses, meaning that this gap is independent of the number of parts. Finally, in Theorem 4.3.6, we combine the results of Theorem 4.3.2 and 4.3.5 and show that for a given number of local warehouses, as the number of parts increases the relative gap between $z^{M P}$ and $z^{L P M P}$ with respect to $z^{M P}$ approaches to zero since the absolute gap between $z^{M P}$ and $z^{L P M P}$ grows faster than $z^{M P}$. Hence, this shows that the Lagrangian dual bound for problem $P$ is asymptotically tight in the number of parts. Under the assumptions given above, we show that the following propositions hold for every realization of random parameters $c_{i}, K_{i}, \lambda_{i n}$ and $W_{n}^{\max }$ for each $i \in I$ and each $n \in N$.

The following lemma shows that for any part $i \in I$, the cost associated with each column generated through the column generation algorithm is bounded below by the optimal objective function value of the EOQ model with unit backorder cost of $\theta_{0}$, 
i.e., $z_{i}^{E O Q\left(\theta_{0}\right)}=\sqrt{\frac{2 K_{i} \lambda_{i 0} c_{i} h \theta_{0}}{c_{i} h+\theta_{0}}}($ Gallego 1998).

Lemma 4.3.1 For a given value of $\theta_{0}, C_{i l} \geq z_{i}^{E O Q\left(\theta_{0}\right)}$ for each $i \in I$ and $l \in L$.

Proof. In problem $S P_{i}(\vec{\theta})$ let $\theta_{n}=0$ for all $n \in N$, meaning that no penalty cost is incurred due to backorders at each local warehouse $n \in N$. This yields an optimal solution in which $S_{i n}=0$ for each $i \in I$ and $n \in N$ and the optimal expected inventory holding and backorder cost at each local warehouse is zero. In this situation, the subproblem $S P_{i}(\vec{\theta})$ is reduced to a single-echelon batch ordering problem with expected cost function $c_{i} h E\left[I_{i 0}\left(Q_{i}, R_{i}\right)\right]+\theta_{0} E\left[B_{i 0}\left(Q_{i}, R_{i}\right)\right]+\frac{\lambda_{i 0} K_{i}}{Q_{i}}$ (Zheng 1992, Gallego 1998). Note that for a given value of $\theta_{0}$, the solution of this single-echelon batch ordering problem is a lower bound to the optimal objective function value of $\operatorname{SP}_{i}(\vec{\theta})$. Furthermore, it is known that the optimal objective function value of the EOQ model with backorders, i.e., $z_{i}^{E O Q\left(\theta_{0}\right)}$, is a lower bound to the solution of the single-echelon batch ordering problem whose cost function is given above (Gallego 1998). Combining these two arguments we establish $C_{i l} \geq z_{i}^{E O Q\left(\theta_{0}\right)}$ for each $i \in I$ and $l \in L$.

Theorem 4.3.2 The optimal objective function value of $M P, z^{M P}$, is in $\mathbf{\Omega}(|I|)$, i.e., $z^{M P}$ is asymptotically bounded below by a function in the order of $|I|$ with probability 1.

Proof. Using the result of Lemma 4.3.1 in constraint (3.13), we have $\sum_{l \in L} C_{i l} x_{i l} \geq$ $z_{i}^{E O Q\left(\theta_{0}\right)}$ for each $i \in I$. Then, by summing up these expressions over all $i \in I$, we obtain $\sum_{i \in I} \sum_{l \in L} C_{i l} x_{i l} \geq \sum_{i \in I} z_{i}^{E O Q\left(\theta_{0}\right)}$. Let $C_{a v e}$ be the average of $z_{i}^{E O Q\left(\theta_{0}\right)}$ over all $i \in I$, hence, defined as $C_{a v e}(I)=\frac{1}{|I|} \sum_{i \in I} z_{i}^{E O Q\left(\theta_{0}\right)}$, then, we simply have $\sum_{i \in I} \sum_{l \in L} C_{i l} x_{i l} \geq|I|$. $C_{\text {ave }}(I)$. Since this holds for all feasible solutions for problem $M P$, we also have $z^{M P} \geq|I| \cdot C_{a v e}(I)$. Furthermore, since we define each $c_{i}, K_{i}, \lambda_{i 0}, \theta_{0}$ as an independent and identically distributed random variable, $z_{i}^{E O Q\left(\theta_{0}\right)}$ is an independent and identically distributed random variable for each $i \in I$ as well. From the convergence of random variables, $C_{\text {ave }}(I)$ converges to a constant with probability 1 as $|I|$ goes to infinity. This suffices to show that $z^{M P}$ is asymptotically bounded below by a function in the order of $|I|$ with probability 1 . 


\section{Lemma 4.3.3}

a) The column generation method yields finite solutions (columns).

b) The total cost associated with each column generated by the column generation method is finite and increases only with the order of $|N|$.

Proof. Proof of part (a): The proof is rather intuitive. Since $K_{i}<\infty, c_{i} h>0$, $W_{n}^{\max }>0$ and $\lambda_{i n} T_{i n}<\infty, S P_{i}(\vec{\theta})$ is guaranteed to yield finite solutions, e.g., for each $n \in N \cup\{0\}, W_{n}^{\max }>0$ implies $\theta_{n}<\infty$ since $\theta_{n}$ has correspondence with $W_{n}^{\max }$ through $\alpha_{n}$, the dual price for the relevant constraint (3.12), and this is necessary to have finite $R_{i}$ and $S_{i n}$. Hence, our column generation method yields finite columns.

Proof of part (b): Provided that the objective function parameters in $\operatorname{SP}_{i}(\vec{\theta})$ are finite, e.g., $K_{i}<\infty, c_{i} h<\infty, W_{n}^{\max }>0$, i.e., $\theta_{n}<\infty$, the optimal objective function value of $S P_{i}(\vec{\theta})$ is finite. Hence, the total cost associated with each column generated by the column generation method is finite. The proof of the second part relies on that due to the decomposition of problem $C G$ into parts, the size of each subproblem $S P_{i}(\vec{\theta})$ grows only with the order of $|N|$.

Lemma 4.3.4 The optimal solution of LPMP contains at most $|N|+1$ non-integer variables.

Proof. The proof is based on an alternative formulation of problem MP obtained by substituting the equality constraints (3.13) in (3.12): Arbitrarily, we select $x_{i 1}$ and then substitute $x_{i 1}=1-\sum_{l \in L^{\prime}} x_{i l}$ in (3.12) for each $i \in I$, where $L^{\prime}=L-\{1\}$. In this way, we establish the alternative formulation $A P$.

Problem AP:

$$
\begin{aligned}
& \operatorname{Min} Z=\sum_{i \in I} \sum_{l \in L^{\prime}} C_{i l} x_{i l} \\
& \begin{array}{l}
\text { s.t. } \\
\sum_{i \in I} \sum_{l \in L^{\prime}}\left(A_{i l n}-A_{i 1 n}\right) x_{i l} \leq W_{n}^{\max }-A_{i 1 n}, \quad \text { for } \forall n \in N \cup\{0\}, \\
x_{i l}=0 / 1, \quad \text { for } \forall i \in I, \forall l \in L^{\prime},
\end{array}
\end{aligned}
$$

Note that in the optimal solution of the $L P$-relaxation of $A P(L P A P)$, there exist $|N|+1$ basic variables. Furthermore, a variable has a fractional value only if it is a basic 
variable. Hence, the optimal solution of $L P A P$ contains at most $|N|+1$ variables with fractional values. Finally, since $A P$ is exactly the same problem as $M P$, this result also holds for LPMP.

Theorem 4.3.5 The gap between the optimal objective function value of $M P, z^{M P}$, and its LP-relaxation, $z^{L P M P}$, is in $\boldsymbol{O}\left(|N|^{2}\right)$, meaning that the gap is asymptotically bounded above by a function of $|N|^{2}$.

Proof. Our proof consists of two parts. In the first part, we introduce a repair algorithm to generate an integer feasible solution to $M P$ by adjusting only the fractional variables in the solution of $L P M P$. Then in the second part, by using the repair algorithm, we show that the gap between the expected cost obtained by the repair algorithm and the Lagrangian dual solution is asymptotically bounded above by a function of $|N|^{2}$.

The repair algorithm relies on the following observation: For each part $i \in I$, the LPMP yields a solution that is a convex combination of columns generated by the column generation algorithm. Accordingly, the solution for each part $i \in I$ corresponds to either

- an integer solution corresponding to one of the columns generated by the algorithm (pure policy), i.e., one of the variables $x_{i l}$ in constraint (3.13) is 1 while the others are 0 , or,

- a fractional solution that is a mixture of a set of columns generated by the algorithm (randomized policy), i.e., a set of variables $x_{i l}$ in constraint (3.13) have fractional values summing to 1 while others are 0 .

Based on these observations, the following repair algorithm generates an integer solution for each part $i \in I$ whose solution is fractional so that the overall solution still remains feasible. 
The Repair Algorithm:

1. For each part $i \in I$ having an integer solution, do nothing.

2. For each part $i \in I$ having a fractional solution, generate a new solution by taking the maximum value of the policy parameters defined by the columns that constitute the fractional solution, and replace the corresponding fractional solution with this new one. To be more specific, let $m \in I$ be any of those parts whose solution is fractional and $\left(Q_{m}, R_{m}, \vec{S}_{m}\right)$ be the corresponding fractional solution. Then, we replace the fractional solution $\left(Q_{m}, R_{m}, \vec{S}_{m}\right)$ with the solution $\left(\widetilde{Q}_{m}, \widetilde{R}_{m}, \vec{S}_{m}\right)=\left(\max _{k \in \Gamma_{m}}\left\{Q_{m}^{k}\right\}, \max _{k \in \Gamma_{m}}\left\{R_{m}^{k}\right\}, \max _{k \in \Gamma_{m}}\left\{\vec{S}_{m}^{k}\right\}\right)$, where $\Gamma_{m}$ is the set of (integer) columns that constitutes $\left(Q_{m}, R_{m}, \vec{S}_{m}\right)$.

The entire solution is feasible for $M P$, because (1) for each part $i \in I$, the solution generated by the repair algorithm satisfies integrality, (2) for each part $i \in I$, the new solution yields lower $E\left[B_{i 0}\left(Q_{i}, R_{i}\right)\right]$ and $E\left[B_{i n}\left(Q_{i}, R_{i}, S_{i n}\right)\right]$ values for all $n \in N$ than the fractional solution yields. Therefore, just like the former columns, the new solutions are guaranteed to satisfy the constraints (3.12).

After introducing the repair algorithm and our notation, now we begin our proof: First, it is a direct consequence of Lemma 4.3.3 that the additional cost incurred by switching from $\left(Q_{m}, R_{m}, \vec{S}_{m}\right)$ to $\left(\widetilde{Q}_{m}, \widetilde{R}_{m}, \overrightarrow{\widetilde{S}}_{m}\right)$ is finite and bounded above by an order of $N$. Hence, for any part $i \in I$, the solution obtained through the repair algorithm has an additional cost bounded above by a finite value in the order of $|N|$. Furthermore, it follows from Lemma 4.3.3 that we need to reassign at most $|N|+1$ fractional variables to obtain a feasible integer solution for $M P$ by using the solution of $L P M P$. Let the objective function value of this integer feasible solution be $z^{H}$. Then, by combining the arguments above, we establish that $z^{H}-z^{L P}$ is asymptotically bounded above by a function of $|N|^{2}$. Since $z^{H}$ is an upper bound on $z^{M P}$, the result also holds for $z^{M P}$. Hence, $z^{M P}-z^{L P}$ is in $\mathbf{O}\left(|N|^{2}\right)$. This also proves that $z^{H}-z^{L P}$ grows only with an order of $|N|$.

Theorem 4.3.6 For a given number of local warehouses $|N|$, the Lagrangian dual bound for problem $P$ is asymptotically tight in the number of parts $|I|$ with probability 1 . 
Proof. It follows from Theorem 4.3.2 and Theorem 4.3.5 that for any given value of $|N|, \lim _{|I| \rightarrow \infty} \frac{z^{M P}-z^{L P M P}}{z^{M P}} \rightarrow 0$ with probability 1 . Since $P$ and $M P$ are identical problems, we have $z^{P}=z^{M P}$. Also, since the solution of LPMP gives the Lagrangian dual solution of $P, z^{L D}$, we obtain $z^{L D}=z^{L P M P}$. Hence, for any given value of $|N|$, we have $\lim _{|I| \rightarrow \infty} \frac{z^{P}-z^{L D}}{z^{P}} \rightarrow 0$ with probability 1 . This shows that $z^{L D}$ is asymptotically tight in the number of parts (Anily and Federgruen, 1990).

Theorem 4.3.6 shows that the Lagrangian dual bound can be used as a benchmark solution for problem $P$ with large number of parts. Since the size of the problems in practice grows especially with the number of parts (compared to the number of local warehouses), this also shows that the corresponding bound can be used as a benchmark solution for practical problems.

\subsection{Computational Study}

In this section, we conduct an extensive computational study to further explore the performances of the heuristics and the Lagrangian dual bound developed Section 3.2.1. First, the performance of the Lagrangian dual bound is tested against the optimal solution for small-size problems to see how reasonable it is to employ the Lagrangian dual bound as a benchmark solution. Then, the performances of the heuristics, i.e., $S_{1}, S_{2}, S_{3}$ and the Lagrangian heuristic are evaluated relative to the Lagrangian dual bound for larger problems, where this bound yields better results. In our analysis, the expected total cost corresponding to each solution is considered as the performance criterion. The performance of the Lagrangian dual bound is mainly evaluated in terms of the percentage dual gap with the optimal expected total cost, $P G A P$. However, we also consider the absolute dual gap, GAP. Similarly, the performances of the heuristics are mainly evaluated in terms of the percentage cost difference between the solution obtained by the heuristic and the Lagrangian dual bound, $P C D$, but we also consider the absolute cost difference between the solution and the bound, $A C D$. Let $z^{*}$ be the optimal objective function value, $z_{L D}$ be the objective function value of the Lagrangian dual solution, and $z$ be the objective function value of any solution to be tested, then the GAP and the PGAP are computed as GAP $=\left|z_{L D}-z^{*}\right|$ and $P G A P=\frac{\left|z_{L D}-z^{*}\right|}{z^{*}}$, whereas the $P C D$ and the $A C D$ are calculated as $A C D=\left|z-z_{L D}\right|$ 
and $P C D=\frac{\left|z-z_{L D}\right|}{z_{L D}}$.

\subsubsection{Experimental Design}

We consider the following six system parameters as the experimental factors: (i) number of parts, $|I|$, (ii) number of local warehouses, $|N|$, (iii) demand rates, $\lambda_{i n}$, (iv) unit variable costs, $c_{i}$, (v) fixed ordering costs, $K_{i}$, and (vi) target aggregate mean response times at the warehouses, $W_{n}^{\max }$. Since lead time, $T_{i n}$, contributes to the model in the form of lead time demand, $\lambda_{i n} T_{i n}$, we do not consider it as a distinct factor. This also means that we do not distinguish the effect of demand rate from that of lead time demand. Using these factors, we conduct a full factorial experiment to investigate the overall performance of the heuristics and the Lagrangian dual bound and perform an analysis of variance (ANOVA) to investigate (i) the individual effect of each factor on the performance of the heuristics and the Lagrangian dual bound and (ii) the interactions between factors.

To generate the problem instances, we first generate a base case setting. Then, based on this base case setting, we build the testbeds for the experiments. For the base case setting, the following parameters are set identical; lead time at the central warehouse, $T_{i 0}$, across all parts, the target aggregate mean response times at the warehouses, $W_{n}^{\max }$, across all warehouses, the lead times at the local warehouses, $T_{i n}$, across all parts and local warehouses. We assume that the unit variable costs, $c_{i}$, and the fixed ordering costs, $K_{i}$, are nonidentical across all parts, the demand rates, $\lambda_{i n}$, are nonidentical across all parts and warehouses. The fixed ordering cost of each part is generated from a uniform distribution. To represent skewnesses of the demand rates and the unit variable costs across the population of parts, we follow an approach similar to the one described in Thonemann et al. (2002). Following this approach, the demand rates are generated through a two-step procedure: First, a part-specific average demand rate is generated randomly for each part, then by multiplying it with a second random number representing the demand intensity at each warehouse partspecific and location-dependent rates are obtained, whereas the unit variable costs are generated in one step since they are only part-specific. To obtain the part-specific average demand rate for any part $i \in I$, say $v_{i}$, we first randomly generate a contin- 
uous number, $u_{d} \sim U[0,1]$, representing the percentile of part $i \in I$ with respect to demand. Next, we obtain $v_{i}$ from $v_{i}(\lambda)=\frac{\lambda}{\rho_{d}} u_{d}^{\frac{1-\rho_{d}}{\rho_{d}}}$, where $\rho_{d}$ is the demand skewness parameter, and $\lambda$ is the average demand rate of all parts. Similarly, the unit variable cost, $c_{i}$, for any part $i \in I$ is generated from $c_{i}(c)=\frac{c}{\rho_{c}} u_{c} \frac{1-\rho_{c}}{\rho_{c}}$, where $\rho_{c}$ is the cost skewness parameter, $c$ is the average unit variable cost of parts, and $u_{c} \sim U[0,1]$ is the percentile of part $i \in I$ with respect to unit variable costs. In this way, we obtain the part-specific average demand rate, $v_{i}$, and the unit variable cost, $c_{i}$, for each part $i \in I$. Finally, by multiplying $v_{i}$ with a second random number generated for each location from $U[0,2]$, we obtain the part-specific location-dependent demand rate, $\lambda_{i n}$, at each warehouse $n \in N$. To obtain the part-specific location-dependent demand rate $\lambda_{i 0}$ for each part $i \in I$ at the central warehouse, we first generate the corresponding external demand rate, $\lambda_{i 0}^{e}$, the same way we generate $\lambda_{i n}$. After obtaining $\lambda_{i 0}^{e}$ and $\lambda_{i n}$ for all $n \in N, \lambda_{i 0}$ is obtained from $\lambda_{i 0}=\lambda_{i 0}^{e}+\sum_{n \in N} \lambda_{i n}$. For any given part, this ensures the differences in the demand rates among warehouses. However, the demand of each part relative to that of the others remains identical at each warehouse. Note that this corresponds to a practical situation where each warehouse serves a market with a similar demand structure. We refer to this case as the symmetric demand case. However, in different geographical regions or markets, the demand of spare parts relative to each other may differ. In order to represent the demand asymmetry across warehouses, the second multiplier is generated from $U[0,2]$ for each part $i \in I$ and each warehouse $n \in N \cup\{0\}$. We call this second case the asymmetric demand case. Based on the data available for the spare parts systems considered in our work, the demand rate (unit variable cost) skewness is approximated as 20\%/80\% (20\%/90\%), i.e., $\rho_{d}=0.139\left(\rho_{c}=0.097\right)$, meaning that $20 \%$ of the parts represent approximately $80 \%(90 \%)$ of the total demand rate (cost) of parts. Table 4.2 summarizes the base setting used.

For the full factorial analysis, we consider 3 levels of the average demand rates, average unit variable costs, average fixed ordering costs and target aggregate mean response times. To generate the problem instances for the experiments, we first generate the base case setting, then we multiply the value of each parameter in the base case setting by the multiplier associated with each level in Table 4.3. Furthermore, to avoid explosion of the number of problem instances, we consider 2 levels of the number of 
parts and the number of local warehouses. For the first set of experiments, we consider small-size problems; the number of parts is set to 4 and 8 , and the number of local warehouses is set to 2 and 4 . In the second set of experiments, in which we experiment with larger problems, the number of parts is set to 100 and 500, and the number of local warehouses is set to 4 and 9 .

Table 4.2: Base case setting for the experiments.

\begin{tabular}{|l|c|}
\hline \multicolumn{1}{|c|}{ Parameters } & Values \\
\hline$\lambda_{\text {in }}$ (units/days) & $\lambda=0.015$ \\
$c_{i}$ (\$/unit) & $c=3000$ \\
$K_{i}$ (\$/order) & $\mathrm{U}[50,100]$ \\
$W_{0}^{\max }$ (days) & 0.3 \\
$W_{n}^{\max }$ (days) & 0.3 \\
$h$ (per year) & 0.25 \\
$T_{i 0}$ (days) & 10 \\
$T_{i n}$ (days) & 1 \\
\hline
\end{tabular}

Table 4.3: Multipliers for the average demand rates, average unit variable cost of parts, average fixed ordering costs and target aggregate mean response times..

\begin{tabular}{|c|c|c|}
\hline Parameters & Number of Levels & Level Multipliers \\
\hline$\lambda_{i n}$ & 3 & $1 / 3,1,10 / 3$ \\
$c_{i}$ & 3 & $1 / 3,1,10 / 3$ \\
$K_{i}$ & 3 & $1 / 3,1,10 / 3$ \\
$W_{n}^{\max }$ & 3 & $1 / 3,1,3$ \\
\hline
\end{tabular}

Based on these, 20 random problem instances are generated for each of the $243\left(3^{4} \times\right.$ $2^{2}$ ) different settings, resulting in a total of 6480 problem instances for each set of experiments. 
In addition to the full factorial analysis, we also carry out sensitivity analysis to precisely observe the effect of each factor on the performance of the heuristics and the Lagrangian dual bound. The problem instances for the sensitivity analysis are generated by using the base case setting in Table 4.2 in a similar way that the testbeds for the factorial analysis are generated. The results that we present here are based on those of the experiments with problem instances with symmetric demand structure. We also experiment with problem instances with asymmetric demand structure. We report the results of the latter only when there is an inconsistency between these two settings. In our experiments, we consider the cases in which (1) only external customers, (2) both type of customers are incorporated in evaluating the performance of the central warehouse. The experiments do not reveal any significant difference between the results of the two cases (in the symmetric demand case both models are the same). Therefore we only present the results for the former case, which is more common. In all experiments, the inventory carrying charge is taken as $25 \%$ annual. The algorithms are coded in $\mathrm{C}++$ and the experiments are run on an Intel $3 \mathrm{GHz}$ processor with 3.5 GB RAM. In the remainder of this section the results of the experiments are presented and discussed.

\subsubsection{Performance of the Lagrangian Dual Bound}

A summary of the results regarding the factorial experiment to test the performance of the Lagrangian dual bound is given in Table 4.4. The main findings are as follows:

- As depicted in Table 4.4, both the average and the maximum PGAP are high, however, both improve when the number of parts is larger.

- Table 4.4 also indicates that the results are sensitive to the factors considered. According to the ANOVA results, all the parameters are found to be significant at 0.05 significance level. The results also show that the parameters highly interact. The most significant interaction effects are the interactions between the number of parts and the average demand rate, the number of parts and target aggregate mean response time and the average demand rate and target aggregate mean response time, each having a p-value of 0.000 . 
The effect of the number of parts on the performance of the Lagrangian dual bound deserves further attention since it is used as a benchmark solution in the second part of experiments, in which we experiment with larger number of parts. Therefore, we carry out a sensitivity analysis to observe the effect of number of parts more deeply. We examine 9 cases with $|I|=10,15,20,25,30,35,40,45,50$, in each of which $|N|=$ 2. For each case, we generate 5 random problem instances using the base case setting in Table 4.2. Figure 4.3 shows the results of the sensitivity analysis. Each point in the figure represents the average of $P G A P$ s for 5 problem instances. As shown in this figure, the performance of the Lagrangian dual bound improves with the number of parts. Note that this result is consistent with Theorem 4.3.6, in which the Lagrangian dual bound is shown to be asymptotically tight in the number of parts. This result together with Theorem 4.3.6 suggests that the Lagrangian dual bound can confidently be used as a benchmark solution in the experiments with larger problems, which will be the case in the remaining of this chapter. Considering the difficulties encountered in evaluating the performance of heuristics for large industry-size multi-item twoechelon inventory systems in the literature (Çağlar et al. 2004, Al-Rifai and Rossetti 2007), this finding makes a significant contribution to the relevant literature.

\subsubsection{Performance of the Lagrangian Heuristic}

The results of the experiments are summarized in Table 4.5. Based on the results, we make the following observations:

- As shown in Table 4.5, the performance of the Lagrangian heuristic is quite satisfactory. The average $P C D$ obtained by the Lagrangian heuristic is less than $1 \%$. This result is even better for problem instances with large number of parts. When the number of parts is 500 , the $P C D$ obtained by the Lagrangian heuristic is less than $1 \%$ for all of the 3240 problem instances studied.

- The ANOVA results reveal that the main effects of all the parameters except the number of local warehouses are significant at 0.05 significance level, each having a p-value of 0.000. On the other hand, the effect of the number of local warehouses on the performance of the Lagrangian heuristic is insignificant in terms of the $P C D$, but significant in terms of the $A C D$. The most significant 
Table 4.4: Effect of the parameters on the performance of the Lagrangian dual bound.

\begin{tabular}{|c|c|c|c|c|c|}
\hline \multirow{2}{*}{\multicolumn{2}{|c|}{ Parameters }} & \multicolumn{2}{|c|}{ GAP } & \multicolumn{2}{|c|}{ PGAP } \\
\hline & & \multirow{2}{*}{$\begin{array}{l}\text { Avg. } \\
524.6 \\
\end{array}$} & \multirow{2}{*}{$\begin{array}{c}\text { Max. } \\
26662.5 \\
\end{array}$} & \multirow{2}{*}{$\begin{array}{l}\text { Avg. } \\
3.84 \% \\
\end{array}$} & \multirow{2}{*}{$\begin{array}{c}\text { Max. } \\
53.87 \% \\
\end{array}$} \\
\hline All instances & & & & & \\
\hline \multirow{2}{*}{ Number of Parts } & 4 & 556.5 & 26662.5 & $4.48 \%$ & $53.87 \%$ \\
\hline & 8 & 492.7 & 13918.0 & $3.21 \%$ & $32.20 \%$ \\
\hline \multirow{2}{*}{ Number of LWHs } & 2 & 456.1 & 19033.5 & $4.06 \%$ & $53.87 \%$ \\
\hline & 4 & 592.2 & 26662.5 & $3.63 \%$ & $46.16 \%$ \\
\hline \multirow{3}{*}{$\begin{array}{c}\text { Average Demand Rate } \\
\text { (units/day) }\end{array}$} & 0.005 & 517.6 & 19033.5 & $5.17 \%$ & $53.87 \%$ \\
\hline & 0.015 & 472.3 & 13918.0 & $3.42 \%$ & $31.68 \%$ \\
\hline & 0.05 & 610.1 & 26662.5 & $2.37 \%$ & $21.17 \%$ \\
\hline \multirow{3}{*}{$\begin{array}{c}\text { Average Unit Cost } \\
\text { (\$/unit) }\end{array}$} & 1000 & 106.4 & 1907.4 & $2.73 \%$ & $27.59 \%$ \\
\hline & 3000 & 305.3 & 10331.5 & $3.76 \%$ & $39.39 \%$ \\
\hline & 10000 & 1147.8 & 26662.5 & $5.02 \%$ & $53.87 \%$ \\
\hline \multirow{3}{*}{$\begin{array}{l}\text { Average Fixed Ordering } \\
\text { Cost (\$/order) }\end{array}$} & 25 & 521.5 & 19021.2 & $5.07 \%$ & $53.87 \%$ \\
\hline & 75 & 569.0 & 24202.5 & $3.86 \%$ & $40.88 \%$ \\
\hline & 250 & 486.5 & 26662.5 & $2.71 \%$ & $27.59 \%$ \\
\hline \multirow{3}{*}{$\begin{array}{l}\text { Target Aggregate Mean } \\
\text { Response Time (day) }\end{array}$} & 0.1 & 509.4 & 10919.8 & $2.82 \%$ & $22.24 \%$ \\
\hline & 0.3 & 839.6 & 26662.5 & $4.49 \%$ & $29.02 \%$ \\
\hline & 0.9 & 308.9 & 13637.6 & $4.26 \%$ & $53.87 \%$ \\
\hline
\end{tabular}


Table 4.5: Effect of the parameters on the performance of the Lagrangian heuristic.

\begin{tabular}{|c|c|cc|cc|}
\hline \multicolumn{2}{|c|}{ Parameters } & \multicolumn{2}{c|}{ ACD } & \multicolumn{2}{c|}{ PCD } \\
\cline { 3 - 6 } & & Avg. & Max. & Avg. & Max. \\
\hline All instances & & 2686.7 & 94984.1 & $0.61 \%$ & $10.33 \%$ \\
\hline \multirow{2}{*}{ Number of Parts } & 100 & 3206.3 & 94984.1 & $1.08 \%$ & $10.33 \%$ \\
\hline \multirow{2}{*}{ Number of LWHs } & 500 & 2167.1 & 39792.7 & $0.14 \%$ & $0.93 \%$ \\
\hline \multirow{2}{*}{ Average Demand Rate } & 4 & 2063.2 & 37882.6 & $0.61 \%$ & $8.16 \%$ \\
(units/day) & 0.005 & 2910.3 & 51784.2 & $0.83 \%$ & $8.16 \%$ \\
\hline Average Unit Cost & 0.015 & 2785.2 & 94984.1 & $0.62 \%$ & $10.33 \%$ \\
(\$/unit) & 0.05 & 2364.6 & 57048.9 & $0.38 \%$ & $4.94 \%$ \\
\hline \multirow{2}{*}{ Average Fixed Ordering } & 1000 & 586.0 & 7065.2 & $0.48 \%$ & $8.16 \%$ \\
Cost (\$/order) & 3000 & 1699.1 & 17733.2 & $0.60 \%$ & $7.53 \%$ \\
& 10000 & 5774.9 & 94984.1 & $0.75 \%$ & $10.33 \%$ \\
\hline Target Aggregate Mean & 25 & 2713.2 & 94984.1 & $0.74 \%$ & $10.33 \%$ \\
Response Time (day) & 0.3 & 2420.7 & 35131.3 & $0.63 \%$ & $7.82 \%$ \\
& 0.9 & 780.2 & 9147.9 & $0.41 \%$ & $7.45 \%$ \\
\hline
\end{tabular}




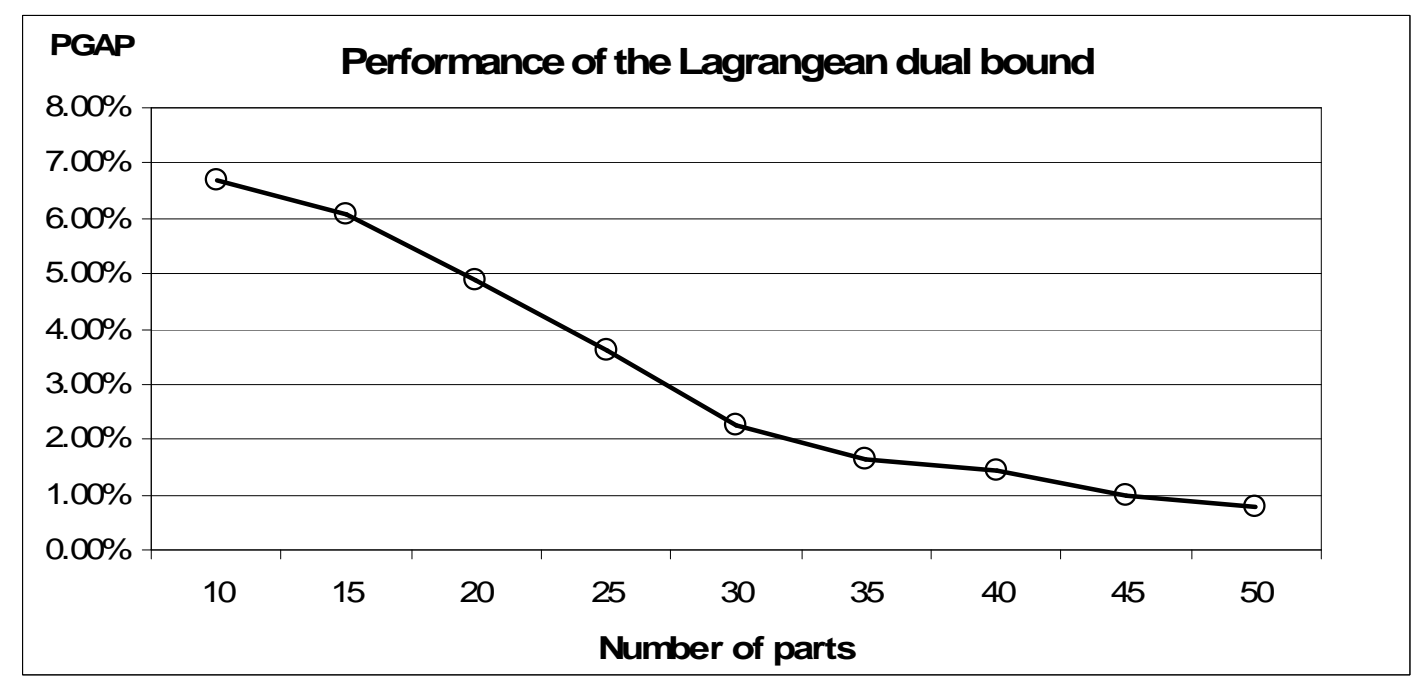

Figure 4.3: Effect of number of parts on the performance of the Lagrangian dual bound.

interaction effects are the interactions of the number of parts with the average demand rate, the average unit variable cost, the average fixed ordering cost and the target aggregate mean response time and those of the target aggregate mean response time with the average demand rate and the number of local warehouses. Each of these interactions is significant with a p-value of 0.000 .

To identify the effect of parameters more precisely, we perform a sensitivity analysis for each factor. Figure 4.4 show the results of the sensitivity analysis for the average demand rate, average unit variable cost, average fixed ordering cost and target aggregate mean response time, whereas Figure 4.5 illustrates the results of the analysis for the number of parts. As shown in Figure 4.4, we consider 10 levels for each of the average demand rate $(\lambda=0.005,0.010, \ldots, 0.050)$, the average unit variable cost $(c=$ $1000,2000, \ldots, 10000)$, the average fixed ordering cost $(K=25,50, \ldots, 250)$ and the target aggregate mean response time $\left(W_{n}=0.1,0.2, \ldots, 1.0\right)$. We also consider two different values of the number of parts $(|I|=50$ and 250), abbreviated as NumPart in Figure 4.4, to explore the interactions between the effect of the number of parts 
and the effects of the corresponding four parameters. As shown in Figure 4.5, for the analysis of the effect of the number of parts, we consider 7 levels with $|I|=50,100$, $250,500,1000,3000$ and 5000. To see the effect of the number of local warehouses in the same figure, we also consider four different values of the number of local warehouses $(|N|=3,6,9$ and 12), abbreviated as NumWare in Figure 4.5. We randomly generate 10 problem instances for each level of the parameters, using the base case setting in Table 4.2. Hence, each point in the figures represents the average of $P C D \mathrm{~s}$ for 10 problem instances. The main observations drawn from the sensitivity analysis are given as follows:

- Figure 4.4 indicate that the Lagrangian heuristic offers a better performance for problem instances with

- high average demand rate,

- low average unit variable cost,

- high average fixed ordering cost,

The results are similar for $A C D$ s and in line with the results of the factorial analysis presented in Table 4.5. We interpret these three observations as follows: Each problem instance conforming the conditions given above corresponds to a situation where the optimal inventory policy parameters, i.e., $Q_{i}, R_{i}$ and $S_{i n}$, are high. Hence, this shows that when the value of optimal policy parameters are high, both the $P C D$ and the $A C D$ obtained by the Lagrangian heuristic decrease. This observation is in line with Wong et al. (2007a) and (2007b).

- The results of the factorial analysis in Table 4.5 shows that the $P C D$ obtained by the Lagrangian heuristic decreases with the number of parts. Figure 4.5 further shows that as the number of parts increases, independent of the number of local warehouses, the $P C D$ obtained by the Lagrangian heuristic approaches to zero.

Note that this observation is similar to our findings regarding the asymptotic behavior of the Lagrangian dual solution. Intuitively, since the solution that the Lagrangian heuristic yields and the Lagrangian dual solution are the primal and dual solutions obtained through Lagrangian relaxation, respectively, it 

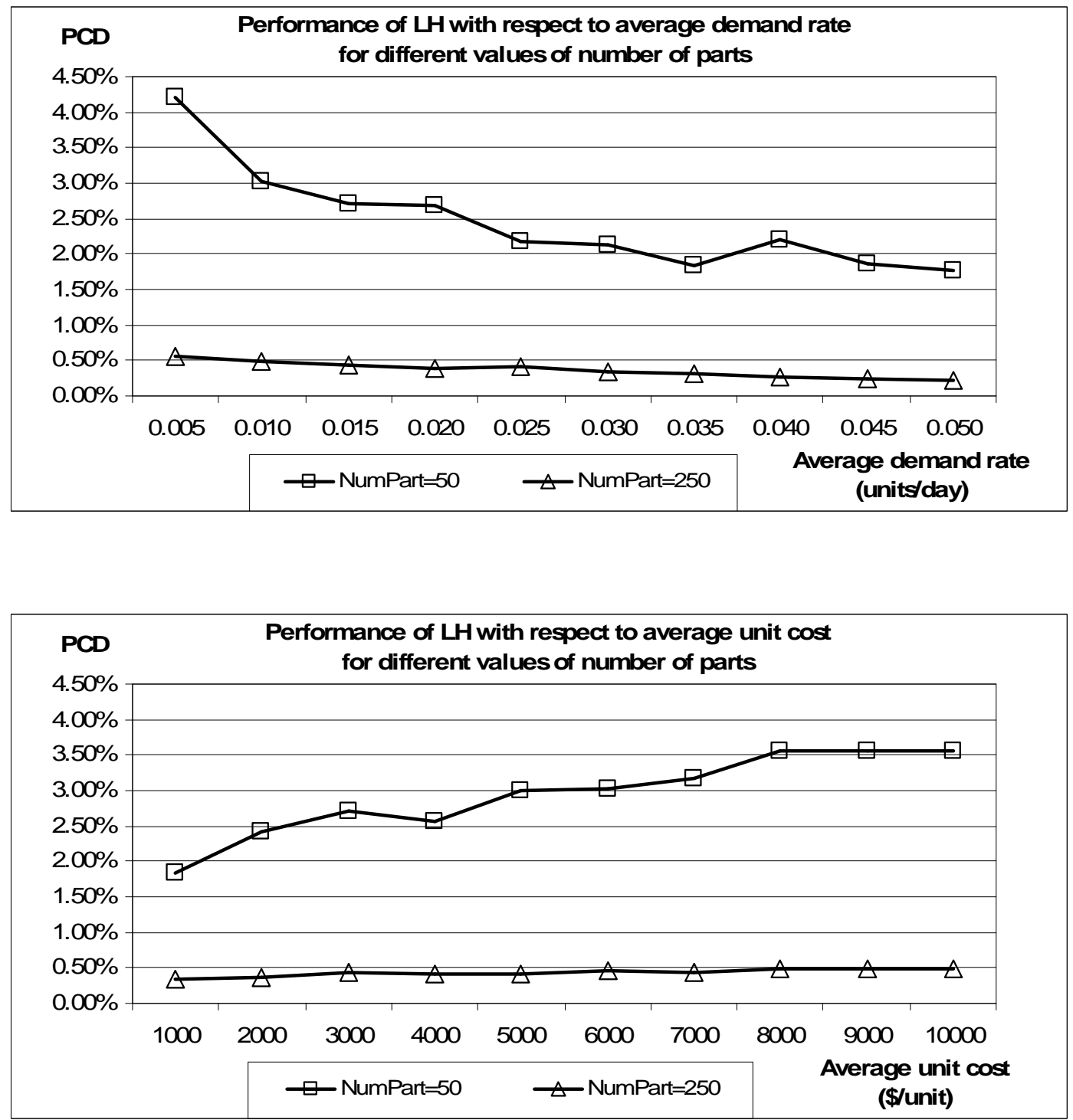

Figure 4.4: Sensitivity Analysis: The effects of parameters on the performance of the Lagrangian heuristic - average demand rates, average unit variable cost of parts, average fixed ordering costs and target aggregate mean response times. 

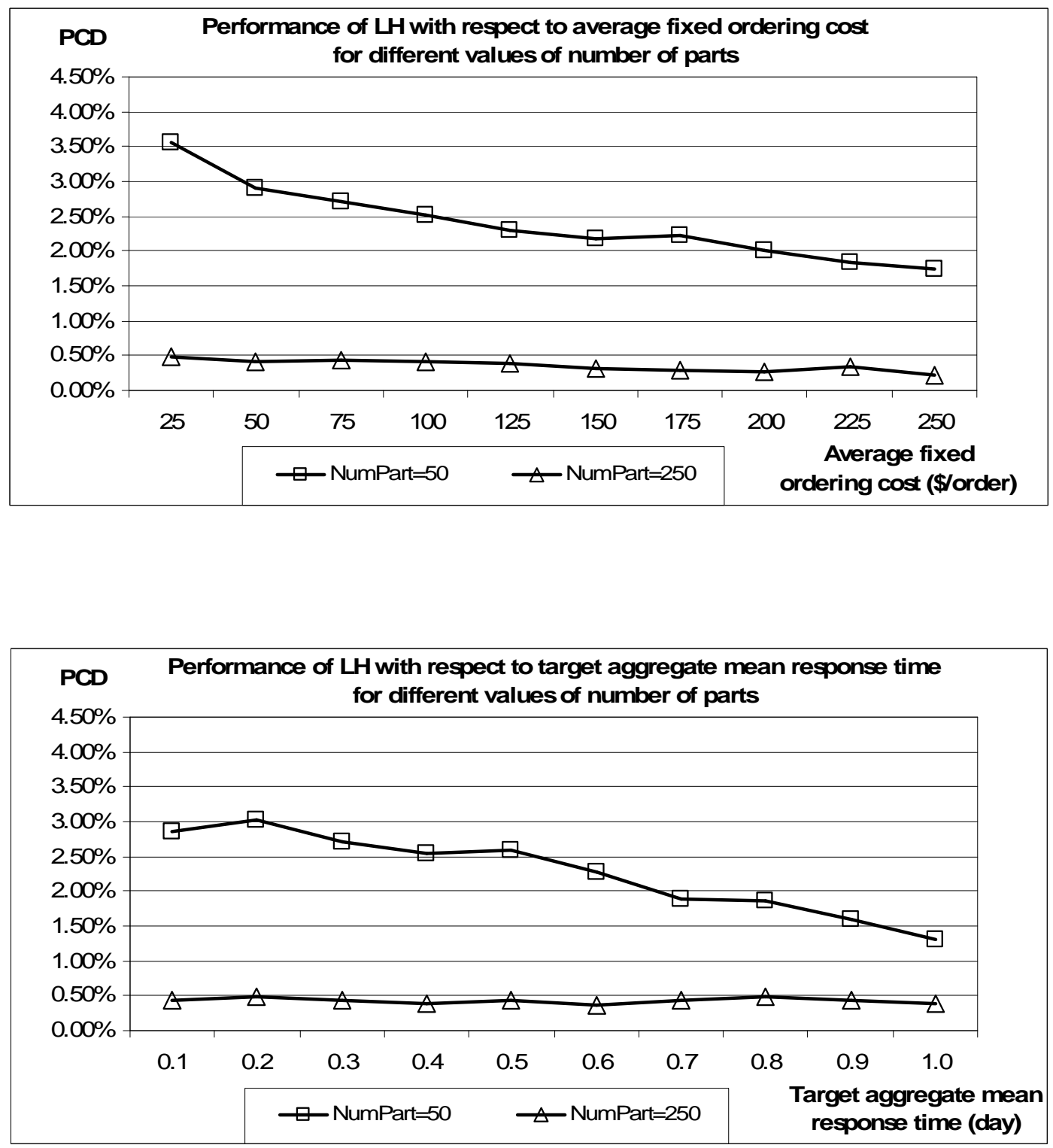

Figure 4.4 (continued) 


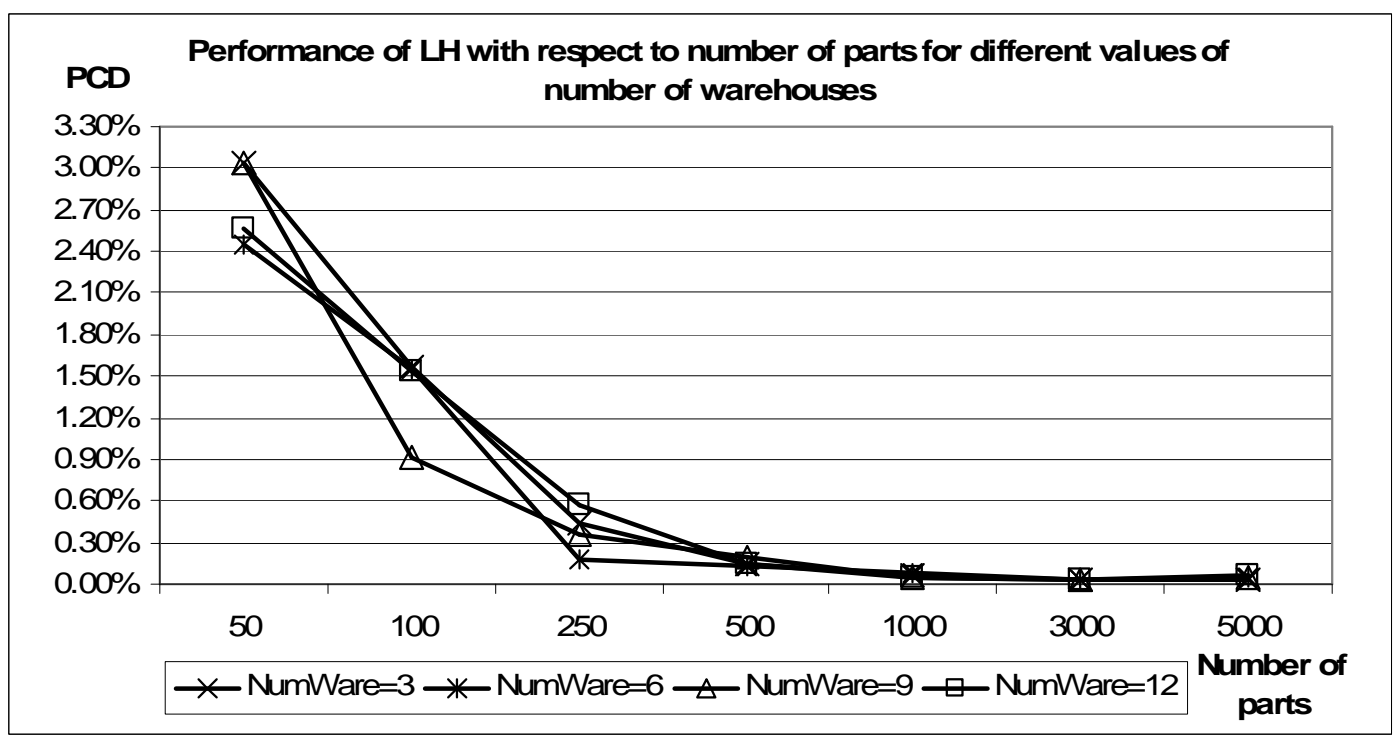

Figure 4.5: Sensitivity Analysis: The effects of parameters on the performance of the Lagrangian heuristic - number of parts, number of local warehouses. 
makes sense to have similar results for both the Lagrangian heuristic and the Lagrangian dual bound. We argue that these two results has some connection with the multi-item approach. Under the multi-item approach, which makes risk pooling possible among parts, as the number of parts increases, the benefits of risk pooling increases. This will also increase the number of alternative near-optimal solutions. In this situation, it is more likely to find a feasible solution that is close to the optimum by using an appropriate heuristic method, e.g., the Lagrangian heuristic. In a similar way, one can find a lower bound for our problem, e.g., the Lagrangian dual bound, by using an appropriate relaxation method, e.g., the Lagrangian relaxation. Similar results exist in the literature for other combinatorial problems as well, e.g., there are greedy algorithms guaranteeing asymptotically optimal solutions for multidimensional knapsack problem and generalized assignment problem (Rinnooy Kan et al. 1993, Romeijn and Morales 2000); the relative gap between the LP relaxation of the knapsack problem and its optimal solution approaches to zero as the number of items increases (Kellerer et al. 2004).

- Figure 4.4 and Table 4.5 also show that the Lagrangian heuristic offers a better performance for problem instances with long target aggregate mean response time. This finding is also in line with our previous observation. Intuitively, long target aggregate mean response times yield loose constraints for the problem $P$. Under the multi-item approach, such loose constraints increase the risk pooling among parts. This increases the number of alternative near-optimal solutions. Hence, under long target aggregate mean response times, it is more likely to find a feasible solution that is close to the optimal solution by using the Lagrangian heuristic.

- Figures 4.4 and 4.5 also indicate that the effect of other parameters vanishes as the number of parts increases. Hence, as the problem gets larger the Lagrangian heuristic becomes more robust.

- In general, we observe that the Lagrangian heuristic yields better results in problem instances with asymmetric demand, and this corresponds to practical situations in which each warehouse serves a distinct market with a different demand structure. This result is in line with Wong et al. (2007b), reporting that 
the greedy heuristic performs better with asymmetric demand instances.

\subsubsection{Performance of the Sequential Heuristics}

The results of the experiments to evaluate the performance of the sequential heuristics $S_{1}, S_{2}$, and $S_{3}$ are summarized in Table 4.6. Accordingly, we make the following observations:

- The average and worst case performances of $S_{2}$ and $S_{3}$ are better than those of $S_{1}$, indicating that the sequential approach performs better when service level requirements are taken into account in calculating the order quantities. However, the results indicate that none of the methods dominates the others. $S_{3}$ outperforms $S_{1}$ and $S_{2}$ in $95.73 \%$ and $70.73 \%$ of all problem instances, respectively, and $S_{2}$ outperforms $S_{1}$ in $92.25 \%$ of all problem instances.

- The average and the maximum $P C D$ s that the sequential approach yield are higher compared to the corresponding results of single-echelon systems operating under $(Q, R)$ policy (Zheng 1992, Axsäter 1996, Silver et al. 1998, Gallego 1998): The $P C D$ obtained by $S_{2}$, which uses $E O Q^{B}$ to determine order quantities, can be as high as $21.75 \%$ in our experiments, while the maximum $P C D$ obtained by the $E O Q^{B}$ in single-echelon systems is $2.90 \%$ (Zheng 1992), empirically, and $11.80 \%$, theoretically (Axsäter 1996). Similarly, the average and the maximum $P C D$ s obtained by $S_{1}$, which uses EOQ, are $5.22 \%$ and $31.03 \%$, respectively, which are fairly high for a batch size heuristic commonly used in practical applications. In contrast to findings for single-item models, our results are comparable with Hopp et al. (1997), who report that the relative gap between the solution that their sequential heuristic yields and a lower bound may reach $15.37 \%$ in multi-item single-echelon inventory systems. Therefore, we conclude that in multi-item systems, which is a more realistic setting for spare parts, the performance of the sequential approach is not as satisfactory as in single-item systems. Although sequential approach is commonly used in practice, the results show that the approach results in very high errors. Note that all sequential heuristics considered in our study utilizes a column generation 
and a greedy algorithm to determine the reorder levels at the central warehouse and the base-stock levels at the local warehouses. However, in practical applications these parameters are typically determined by using simpler methods, which may yield much higher errors than the PCDs presented here. All these indicate that some of the conjectures in the literature about the performance of the sequential approach are misleading, and using the sequential approach in practical applications may not be the best option.

- The Lagrangian heuristic generally performs better than the sequential heuristics. When the number of parts is 100 , the Lagrangian heuristic outperforms $S_{2}\left(S_{3}\right)$ in $95.77 \%(94.51 \%)$ of the problem instances, whereas when the number of parts is 500 , the Lagrangian heuristic outperforms $S_{2}\left(S_{3}\right)$ in $99.88 \%$ $(99.48 \%)$ of the problem instances. This shows that as the number of parts increases, the Lagrangian heuristic, which is based on the simultaneous approach, becomes much more dominant over the sequential heuristics. Intuitively, under the multi-item approach, determining the order quantity of each part independent of the other parts' parameters benefits less from the opportunities of risk-pooling among parts. Therefore, for a system operating under the multiitem approach, (1) as the number of parts increases, or (2) as we switch from single-item to multi-item setting, the performance of the sequential heuristics deteriorates relative to that of the simultaneous approach.

In addition to the observations given above, the ANOVA results indicate the followings:

- The effects of all the parameters considered in the factorial analysis are significant at 0.05 significance level, each having a p-value less than 0.005 . The most significant observation is that the sequential heuristics generally perform better in problem instances with

- high average fixed ordering cost,

- short target aggregate mean response time,

- low average demand rate. 


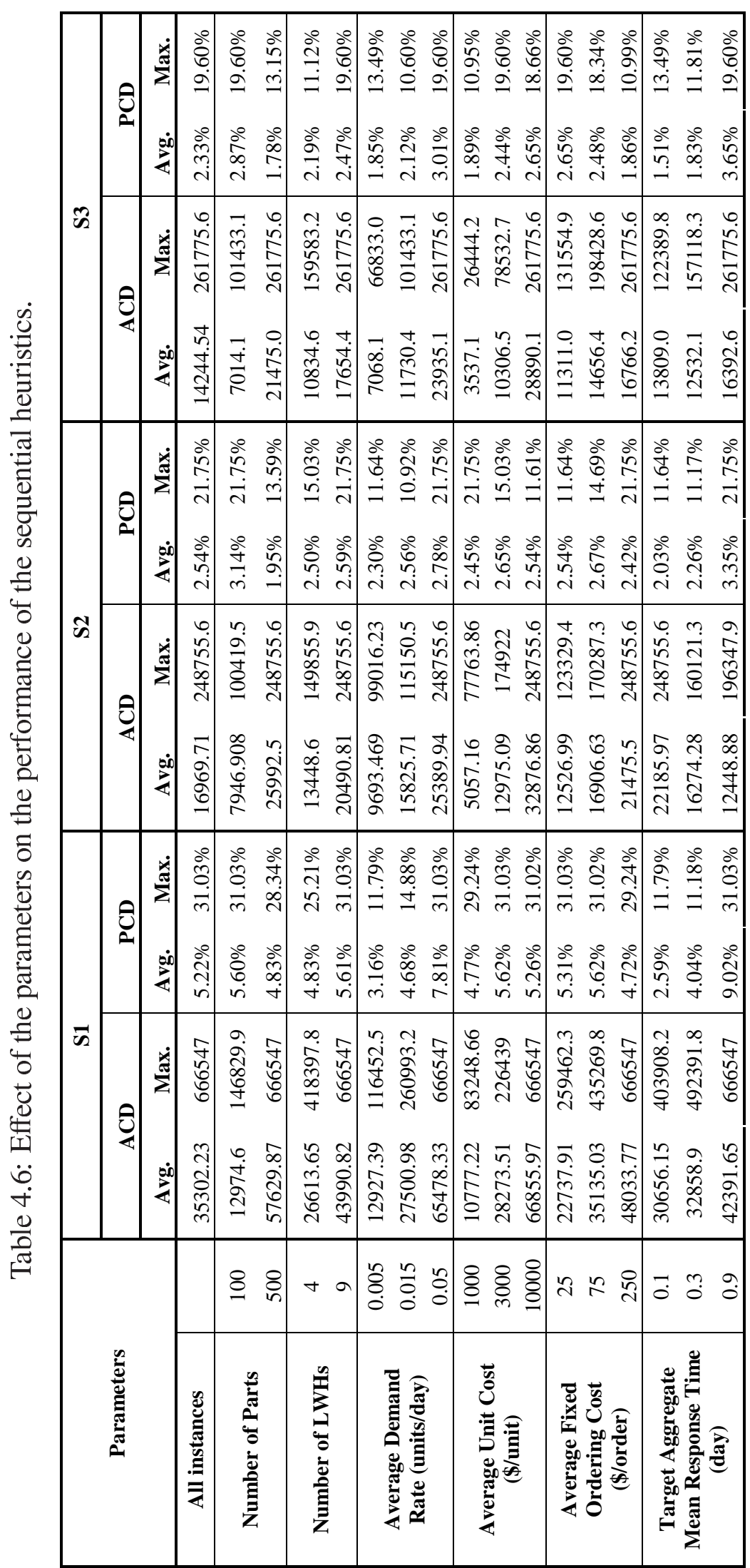


These findings are in line with the literature (Zheng 1992, Hopp et al. 1997). However, even with those problem instances that are in favor of the sequential heuristics, the $P C D$ obtained by the sequential approach is still higher compared to that of using the Lagrangian heuristic. This can be seen also in Table 4.6 .

- Almost all the interactions between the factors are significant at 0.05 level. Especially the average demand rate, the average fixed ordering cost, and the average unit variable cost highly interact with each other. This makes it difficult to draw conclusions only by looking at the results of the individual (main) effects of parameters. Therefore, we also analyze and interpret the effects of parameters considering the interactions. The most critical observation is that the sequential approach performs best either when the average demand rate and the average fixed ordering cost are low, the average unit variable cost is high, and the target aggregate mean response time is short; or when the average demand rate and the average fixed ordering cost are high, the average unit variable cost is low, and the target aggregate mean response time is short. Consistent with this result, the performance of the sequential heuristics relative to that of the Lagrangian heuristics is best when the average demand rate and the average fixed cost are low, the average unit variable cost is high, and the target aggregate mean response time is short. Note that this corresponds to problem instances in which our problem becomes much more tighter and the optimal policy parameters become smaller. We note that this corresponds to instances in which the performance of the Lagrangian heuristic performs worse. The results show that even in this setting, the Lagrangian heuristic outperforms the sequential heuristics in most of instances. Under this specific setting, when the number of parts is 100, the Lagrangian heuristic outperforms $S_{3}\left(S_{2}\right)$ in $70.0 \%$ $(65.0 \%)$ of the problem instances, whereas when the number of parts is 500 , the Lagrangian heuristic outperforms $S_{3}\left(S_{2}\right)$ in $95.5 \%$ (100.0\%) of the problem instances. Again, this shows that as the number of parts increases, the Lagrangian heuristic becomes much more dominant over the sequential heuristics even for the problem instances less favorable for the Lagrangian heuristic. 
We further perform a sensitivity analysis to identify the effect of parameters more precisely. We conduct the experiments based on the testbed used for the sensitivity analysis of the Lagrangian heuristic. The main observations drawn from the sensitivity analysis are given as follows: Figures 4.6 and 4.7 illustrate the result of analysis regarding the effect of the number of parts on the performance of the heuristics based on the $P C D$ s and $A C D$ s, respectively. As shown in Figure 4.6, similar to the findings regarding the performance of the Lagrangian heuristic, as the number of parts increases, the $P C D$ obtained by the sequential heuristics decreases. However, Figure 4.6 also shows that neither of them converges to zero, although that of the Lagrangian heuristic does, e.g., the average $P C D$ obtained by the sequential heuristic with EOQ is $4.38 \%$ even the number of parts is 5,000. Figure 4.7 shows that the $A C D$ obtained by the sequential heuristics increases with the number of parts quite faster than that of the Lagrangian heuristic.

We also consider setting the order quantities to one and, then, applying the sequential approach. This way, we explore the performance of using a base-stock policy approximation to our batch ordering problem. Therefore, we carry out experiments by using the testbed that for the factorial analysis. Based on the results, the performance of the base-stock policy approximation is found to be very poor. Even the average PCP is larger than $105 \%$. As a result, we conclude that in the presence of fixed ordering costs, it is not reasonable to operate under a base-stock policy.

\subsubsection{Computational requirements of the solution procedures and experiments with Practical-Size Problems}

In this section, we test the performance of the heuristics in terms of the computational requirements. Figure 4.8 illustrates the computational requirements of the heuristics that have run to test the effect of the number of parts on the heuristics in Sections 4.4.3 and 4.4.4. The figure shows only the results for $|N|=9$ and 12. As shown in the figure the average CPU time required by heuristics increases with the number of parts. This is rather intuitive. The figure also shows that the average CPU time for the Lagrangian heuristic is higher than those for the sequential heuristics, but they are comparable even when $|I|=5,000$. This indicates the computational savings that 

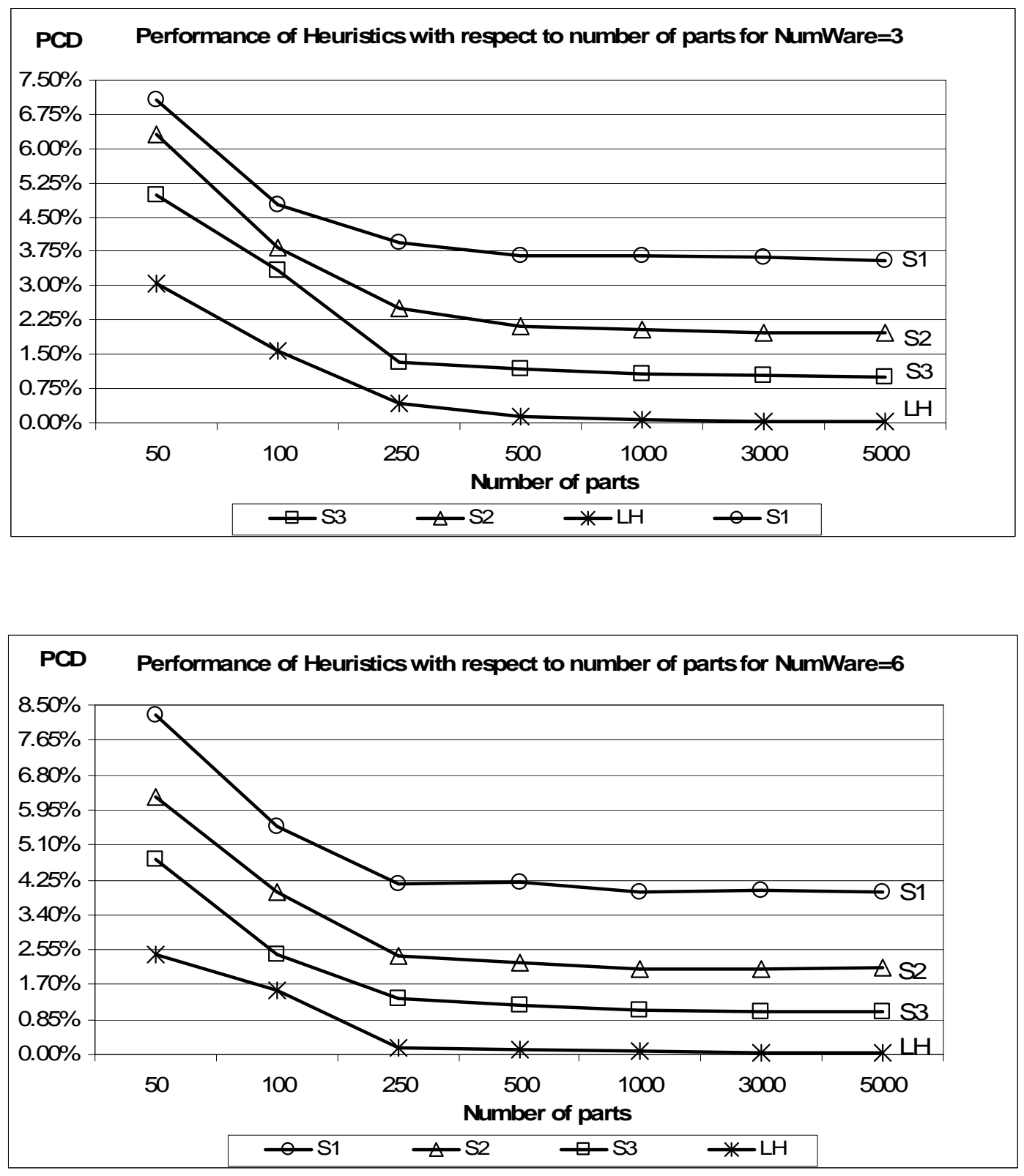

Figure 4.6: Performance of the heuristics with respect to number of parts and number of local warehouses - PCD. 

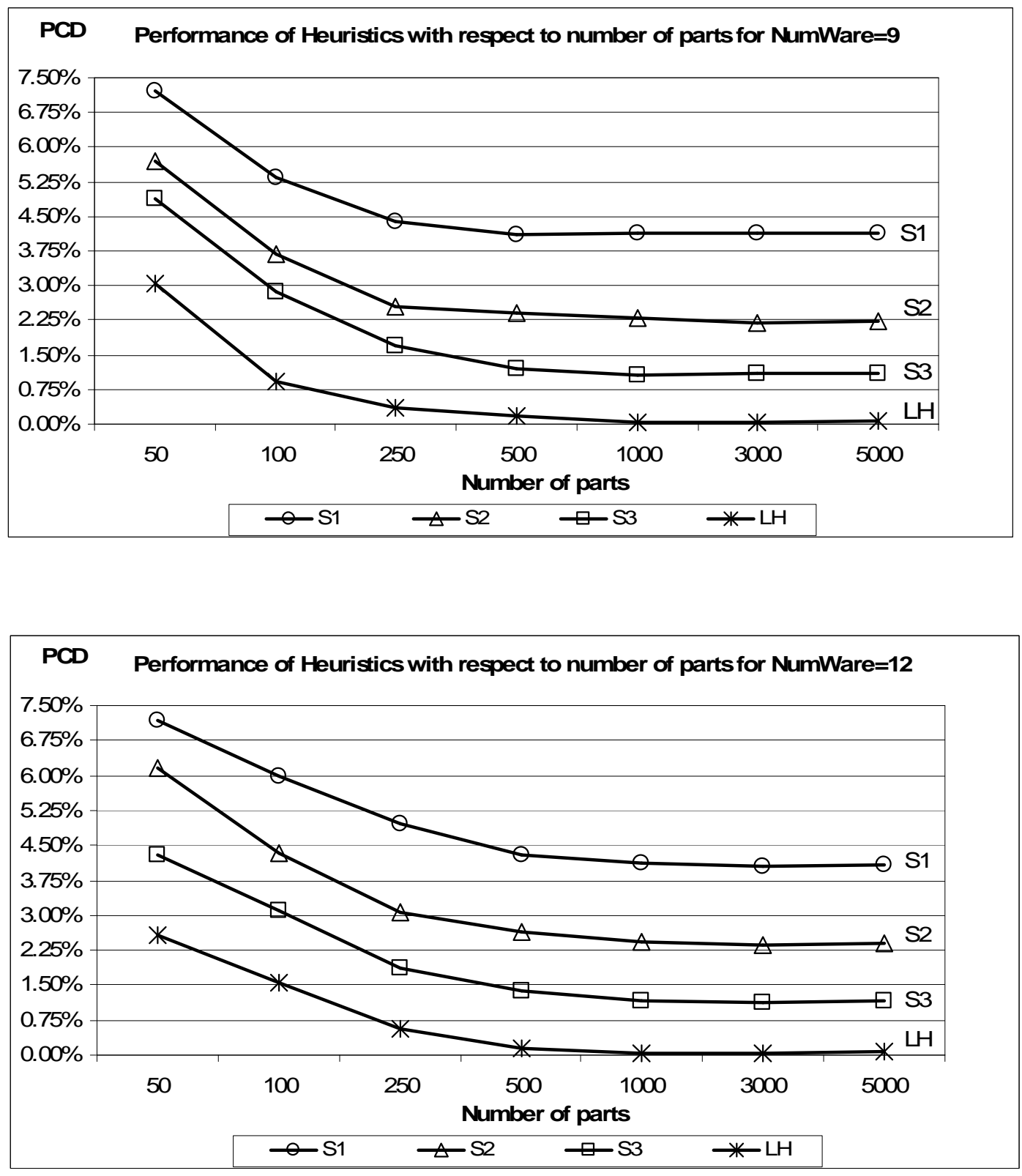

Figure 4.6 (continued) 

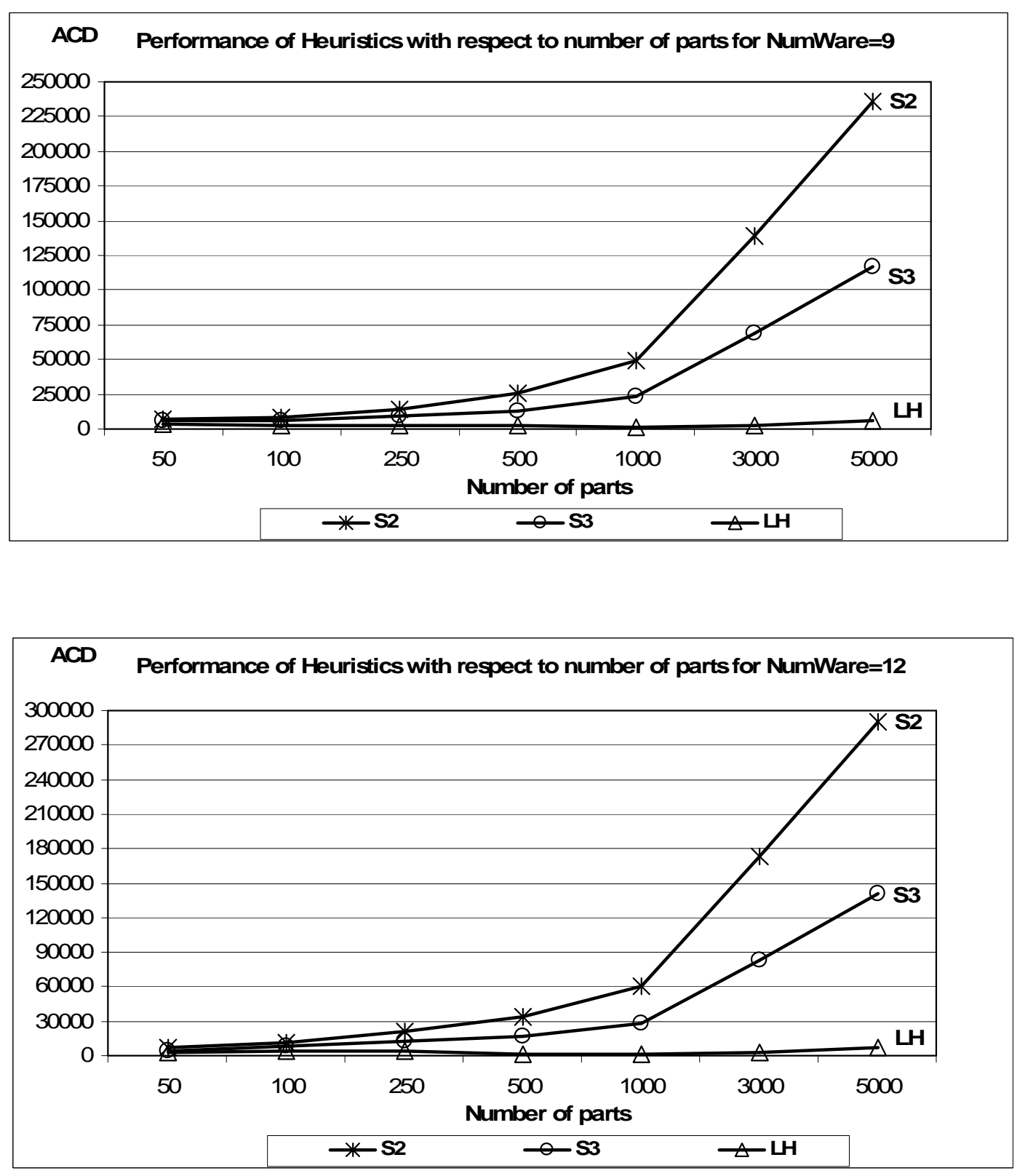

Figure 4.7: Performance of the heuristics with respect to number of parts and number of local warehouses - $A C D$. 
can be obtained by sequential heuristics are quite limited. Recall that by using the sequential heuristics, the computational time required to solve the problem $P$ is reduced by eliminating the outer loop in the single-item two-echelon subroutine in the column generation algorithm. However, the experiments reveal that for small-size problems, the bulk of the computational effort is devoted to the column generation algorithm, whereas for problems with larger number of parts, the most of the computational time is spent by the greedy algorithm. Thus, elimination of the subroutine, which makes sequential heuristics faster, contributes less to computational savings of the overall method for large-scale problems.

Furthermore, we experiment with larger problem instances to further explore the performance of the Lagrangian heuristic in practical-size problems. The number of parts is set to 10000 , and the number of local warehouses is set to 12 . The problem instances are generated by using the base case setting in Table 4.2. We consider both the symmetric and the asymmetric problem instances. The results of the experiment show that the average PCDs obtained by the Lagrangian heuristic are $0.09 \%$ and $0.04 \%$, and the average CPU times required by the Lagrangian heuristic are 15.55 and 11.94 hours for the symmetric and the asymmetric problem instances, respectively.

Although the papers in the literature deal with systems under different settings, a comparison with them is still possible to a certain extent. Hopp et al. (1999) who consider a multi-item two-echelon batch ordering system similar to ours report that their heuristic can solve problem instances with 1263 parts and 2 regional facilities. They also show that for relatively small problem instances (with up to 10 parts and 5 regional facilities) the relative gap between the expected total cost of the solution obtained by the heuristic and the lower bound that they propose is less than 5\%. Compared to Hopp et al. (1999), our heuristics seem to be significantly better both in terms of relative errors and the computational efficiency. When we compare our results with Wong et al. (2007b), who apply a column generation and decomposition method similar to our column generation algorithm for a multi-item two-echelon system under pure base-stock policy, our results seem to be comparable in terms of relative errors. Furthermore, although our problem is more difficult than theirs, our results are significantly better in terms of the computational efficiency, e.g., while they can solve 

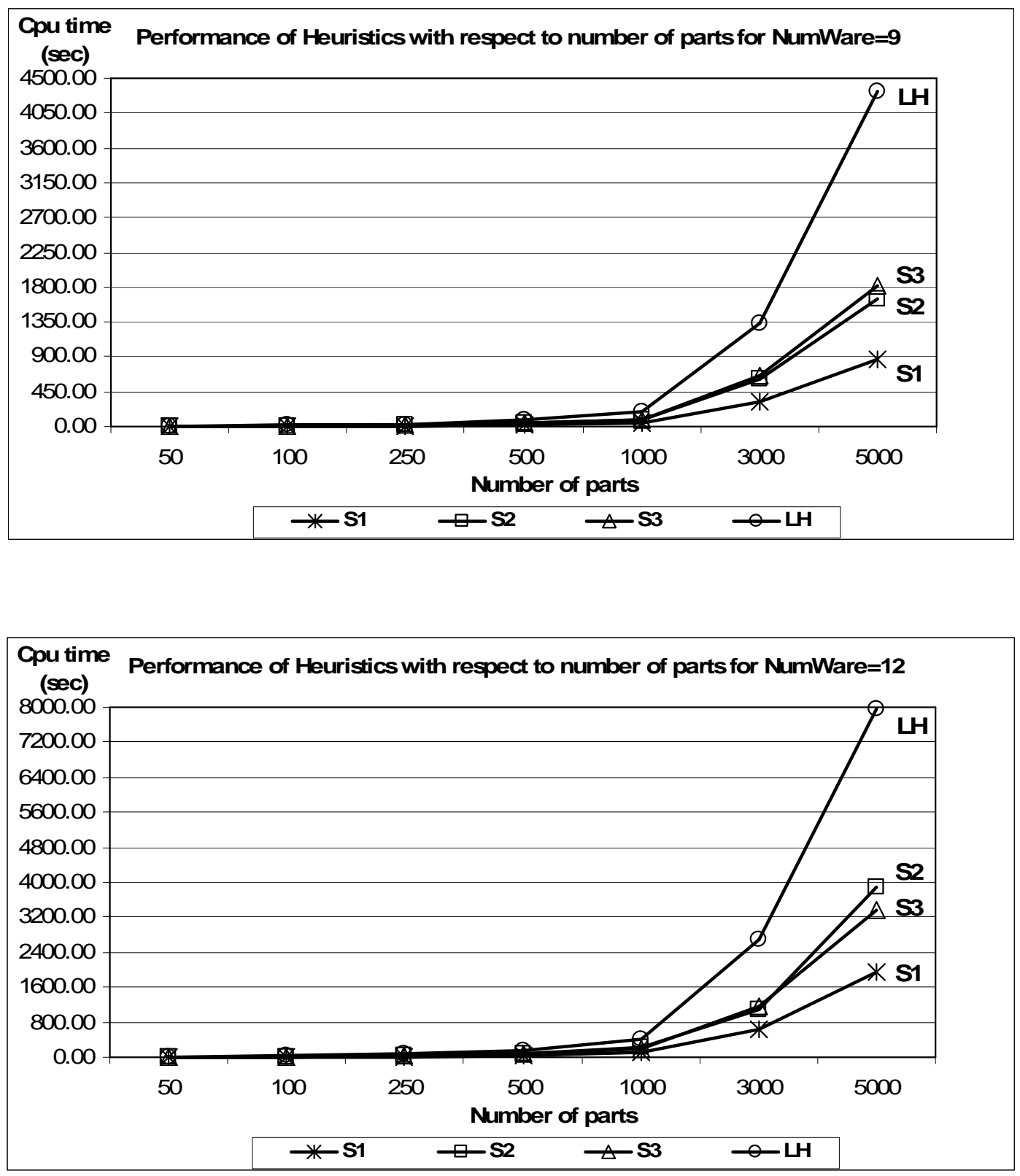

Figure 4.8: Computational requirements of the heuristics with respect to number of parts and number of local warehouses. 
problems problems up to a size of 100 parts, we can solve problems with up to 10,000 parts. Note that when we fix the order quantities at 1 , our model reduces to their system, hence our heuristics are applicable in their setting as well. We also compare our results with Caggiano et al. (2007), who propose heuristics to solve large-scale multi-item multi-echelon systems under base-stock policy. Although their system is very different from our system (our model involves batch ordering decision, while their model is applicable to more than two-echelon), one can see that our results are slightly better than theirs both in terms of relative errors and computational efficiency. For example, while they can solve problems with 27175 part-location combinations in almost 21 hours, using the Lagrangian heuristic, we can solve 130,000 part-location combinations (10,000 parts, 12 local warehouses and 1 central warehouse) within 12 hours. As evident from these comparisons, our work contributes to the relevant literature by proposing an efficient and tractable heuristic for large-scale spare parts inventory problems. As opposed to these papers, a comparison with Al-Rifai and Rossetti (2007) is not possible since the performance of their heuristic is not compared against an analytical solution or a bound, since such a solution or a bound is not available for large-scale problems. Çağlar et al. (2004) encounter a similar problem. They report that the lower bound that they use to test their heuristic is not tight, and hence, the relative gap between their heuristics and the lower bound is used as a conservative estimate of the true relative error. As opposed to these studies, the Lagrangian dual bound for our problem is tractable and performs quite well for larger-scale problems. Hence, our findings regarding the performance of the Lagrangian dual bound also contribute to the relevant literature.

\subsection{Conclusion}

We propose four alternative heuristics to find the optimal or near-optimal policy parameters of a multi-item two-echelon inventory distribution system with batch ordering in the central warehouse. The first heuristic, which we call the Lagrangian heuristic, is based on the simultaneous approach and relies on the integration of a column generation method and a greedy algorithm. The other three heuristics are based on the sequential approach, in which first the order quantities are determined using 
a batch size heuristic, then the reorder levels at the central warehouse and the basestock levels at the local warehouses are determined through the same method used for the Lagrangian heuristic, i.e., a column generation and a greedy algorithm. We explore the performance of the Lagrangian dual bound that we develop in Chapter 3 and analytically show that this bound is asymptotically tight in the number of parts. By making use of the results of the computational study we develop several insights, some of which are summarized as follows: The lower bound for the optimal expected total cost is found to be quite tight, especially when the number of parts is high, e.g., the relative gap between the bound and the optimal expected total cost is less than $1 \%$ even when the number of parts is only 50 . These results together with the asymptotic tightness of the lower bound with the number of parts motivates us using it in further numerical experiments with large number of parts as a benchmark solution. Based on the results of these further experiments, the Lagrangian heuristic performs quite well in terms of the relative difference between the expected total cost of the solution obtained by the heuristic and the lower bound. As the number of parts increases, the performance of the heuristic improves further, making the heuristic very promising for practical applications. The computational requirement of the heuristic is also quite tolerable. To be more specific, the experiment with 10,000 parts and 12 warehouses reveals that the relative cost difference is $0.04 \%$; problems of this size can be solved within 12 hours on an Intel $3 \mathrm{GHz}$ processor with $3.5 \mathrm{~GB}$ RAM. Furthermore, as the number of parts increases, the Lagrangian heuristic becomes robust and becomes insensitive to other parameters and whether the demand is symmetric or not. The heuristic is also quite tractable. It can be used to solve very large practical problems in reasonable computation time. This makes the Lagrangian heuristic very promising for practical applications. The performance of the heuristics that are based on the sequential approach are also satisfactory, but not as much as the Lagrangian heuristic. As the number of parts increases the performance of the sequential approach deteriorates compared to that of the Lagrangian heuristic. Furthermore, the computational advantage of the sequential heuristics is found to be limited compared to the Lagrangian heuristic. The errors in practical applications are expected to be even higher considering that our sequential heuristics involve a column generation method, which is more sophisticated than the methods used in sequential approach applications in practice. Hence, despite the fact that the sequential approach is widely used in prac- 
tice and that its performance is experimentally verified in single-item problems, our work shows that in a multi-item setting and under a multi-item approach, the performance of the sequential approach heuristics is inferior compared to the Lagrangian heuristic, which yields superior results even for large problems in reasonable time. We also find that the performance of the sequential heuristics depends on the batch size heuristic used. The batch size heuristic that take service level requirements into account outperforms the EOQ. This shows that if the sequential heuristics are used to solve batching problems in multi-item two-echelon inventory systems, it is better to take the service level requirements into account in calculating the order quantities. For this purpose, one can use the batch size heuristics that we consider in this chapter.

To summarize, we contribute to the literature by proposing efficient and tractable heuristics to solve large, practical-size multi-item two-echelon inventory control problems with batch ordering at the central warehouse, one of which significantly outperforms the others. The comparisons based on our heuristics also makes a contribution to the literature in evaluating the performance of the sequential approach against the simultaneous approach in a multi-item multi-echelon setting. Our work also contributes to the literature by proposing a tight and efficient lower bound on the optimal total cost for practical-size problems. 


\section{CHAPTER 5}

\section{EXTENSIONS TO COMPOUND POISSON DEMAND}

In this chapter, we extend the heuristics and the lower bound, which are shown to perform quite well for the pure Poisson demand setting, to the compound Poisson demand setting. To the best of our knowledge, our work is the first to propose heuristics for a multi-item two echelon inventory system facing compound Poisson demand. To evaluate the steady-state performance of the system and also the objective function and the constraints of the problem, we consider both an exact and approximate methods. For the approximate evaluation, we propose four alternative methods. The first method is based on the binomial disaggregation, which is exact for the Poisson demand. The second approximation is the extension of the two-moment approximation developed by Gallego et al. (2007) to a batch ordering system. As a third approximation, we extend the two-moment approximation developed by Graves (1985) to the compound Poisson demand setting. The fourth approximation corresponds to the implementation of the METRIC to our inventory system. For the exact evaluation, we adopt the flow-unit method by Axsater (2000). We also show that the Lagrangian dual bound is asymptotically tight in the number of parts. Later, in a computational study, we test the performances of the heuristics against the lower bound, the performance of the approximations against the results obtained by the exact evaluation method. To the best of our knowledge, our work is the first to investigate the performance of the approximations most commonly used in the literature, i.e., the METRIC and the two-moment approximation, for a multi-item two-echelon system facing a compound Poisson demand.

In Section 5.1, we introduce the compound Poisson demand model. We describe the exact and the approximate evaluation methods considered for this model in Sections 
5.2 and 5.3, respectively. Later, in Section 5.4, we explain how we extend the heuristics developed in Chapter 4 to compound Poisson demand. Finally, in Section 5.5, we report and discuss our computational results.

\subsection{The model}

The extensions of the developments in Chapters 3 and 4 to compound Poisson demand setting requires revising the model in Section 3.1. Similar to the model in Section 3.1, we consider a two-echelon distribution network in which the lower echelon consists of a set, $N$, of local warehouses, each is denoted by $n=1,2, \ldots|N|$, while the upper echelon corresponds to a central warehouse, which is denoted by $n=0$. There is a set, $I$, of parts, each is denoted by $i=1,2, \ldots|I|$. In contrast to the Poisson demand model, the external customer demands for part $i$ at the central warehouse and the local warehouse $n \in N$ follow a compound Poisson process with customer arrival rates $\lambda_{i 0}^{e}$ and $\lambda_{i n}$, having demand sizes of $v_{i 0}^{e}$ and $v_{i n}$ with probabilities $P\left(V_{i 0}^{e}=v_{i 0}^{e}\right)$ and $P\left(V_{i n}=v_{i n}\right)$, respectively. As in Section 3.1, the central warehouse also faces internal demands from local warehouses; the internal and external demands are not differentiated and are satisfied according to the FCFS rule. Finally, we assume partspecific holding costs for all facilities and part-specific fixed ordering costs for the central warehouse.

Under the compound Poisson demand, our system operates as follows: Whenever a demand of size $v_{\text {in }}$ for any part $i$ arrives at warehouse $n \in N$, it is immediately satisfied from stocks if there are $v_{\text {in }}$ parts available; otherwise, the unsatisfied portion of demand is backordered. In both cases, if the external demand is directed to a local warehouse, an order of size $v_{\text {in }}$ is placed at the central warehouse so that the inventory position reaches $S_{i n}$. Therefore, this policy is known as order-up-to $S$ policy (Forsberg 1995). This internal request is satisfied within a constant transportation lead time of $T_{i n}$, if the part is available in the central warehouse. Otherwise, the internal demand is backordered as well. In any case, if the inventory position of the central warehouse drops to or below reorder level $R_{i}$, a sufficient number of orders with batch size $Q_{i}$ are placed at the outside supplier so that the inventory position exceeds $R_{i}$. Note that in the pure Poisson demand case, only a single batch of size $Q_{i}$ is placed since the 
demand size is always one. Under compound Poisson demand model, the inventory position at the central warehouse may overshoot the reorder level $R_{i}$ since the demand size is a random variable. Since the number of orders is a variable depending on the overshoot quantity, but the batch size is constant this policy is known as $(n Q, R)$ policy (Axsäter 2000). It is assumed that the supplier has ample stock and can always satisfy requests for part $i$ in a constant lead time of $T_{i 0}$. We also assume that partial backlogging is allowed since this simplifies the analysis.

Based on the system definition, our problem can be stated as in Section 3.1: finding the policy parameters minimizing the sum of the inventory holding and fixed ordering costs subject to constraints on the aggregate mean response time. To formulate this problem, we revise our notation and extend it to compound Poisson setting as it is given in Table 5.1. As in Section 3.1, for sake of brevity, we omit the parameters that the variables depend on, e.g., $I_{i n}\left(t, Q_{i}, R_{i}, S_{i n}\right)$ is simply denoted as $I_{i n}(t)$. Similarly, since our focus is on the limiting behavior of stochastic variables, we also omit the time component, e.g., $I_{i n}=\lim _{t \rightarrow \infty} I_{i n}(t)$. The additional notation for the compound Poisson demand model is as follows: Let $Y_{\text {in }}$ be the number of customer arrivals during lead time $T_{i n}$ and $V_{\text {in }}$ be the size of a demand for each part $i \in I$ at each warehouse $n \in N \cup\{0\}$. Then, the demand during lead time $T_{i n}, D_{i n}$, is expressed in terms of $Y_{i n}$ and $V_{\text {in }}$ as follows: Let $v_{i n}^{k}$ be the size of $k^{\text {th }}$ demand occurred during the lead time $T_{i n}$ at warehouse $n \in N \cup\{0\}$ for part $i \in I$, then $D_{i n}=\sum_{k=1}^{Y_{i n}} v_{i n}^{k}$ for each part $i \in I$ and each local warehouse $n \in N$. Then, the average demand rate per unit time, $\mu_{i n}$, and the average demand during lead time $T_{i n}, E\left[D_{i n}\right]$, are given as $\mu_{i n}=\lambda_{i n} E\left[V_{i n}\right]$ and $E\left[D_{i n}\right]=\lambda_{i n} T_{i n} E\left[V_{i n}\right]$, respectively for each part $i \in I$ at warehouse $n \in N$. Similarly, the external demand at the central warehouse during lead time $T_{i 0}, D_{i 0}^{e}$, is expressed in terms of $Y_{i n}^{e}$ and $V_{i n}^{e}$ for each part $i \in I$, where $Y_{i n}^{e}$ and $V_{i n}^{e}$ are the corresponding variables associated with the external customers. Then, the average external demand rate per unit time, $\mu_{i 0}^{e}$, and the average external demand during lead time $T_{i 0}, E\left[D_{i 0}^{e}\right]$, at the central warehouse are given as $\mu_{i 0}^{e}=\lambda_{i 0}^{e} E\left[V_{i 0}^{e}\right]$ and $E\left[D_{i 0}^{e}\right]=$ $\lambda_{i 0}^{e} T_{i 0} E\left[V_{i 0}^{e}\right]$, respectively for each part $i \in I$. Based on the definitions, the demand per unit time for part $i \in I$ at warehouse $n \in N$ has a compound Poisson distribution with mean $\mu_{i n}=\lambda_{i n} E\left[V_{i n}\right]$ and $\sigma_{i n}^{2}=\lambda_{i n} E\left[V_{i n}^{2}\right]$ (Axsäter 2006). Similarly, the external demand per unit time for part $i \in I$ at the central warehouse has a compound Poisson 
distribution. By using $\mu_{i 0}^{e}$ and $\mu_{i n}$, the average demand rate per unit time at the cental warehouse, $\mu_{i 0}$, is defined as $\mu_{i 0}=\mu_{i 0}^{e}+\sum_{n \in N} \mu_{i n}$ for each part $i \in I$.

An important parameter, not for the evaluation of the system, but for the experimental analysis is the variance-to-mean ratio, simply denoted by $\frac{\sigma_{i n}^{2}}{\mu_{i n}}$. The main difference between the pure Poisson demand and the compound Poisson demand lies on the $\frac{\sigma_{i n}^{2}}{\mu_{i n}}$ ratio. The compound Poisson demand distribution makes it possible to have a demand with $\frac{\sigma_{i n}^{2}}{\mu_{i n}} \geq 1$, while for the Poisson demand this ratio is strictly 1 . In a way, for the rest of chapter, we extend the results for pure Poisson case, i.e., $\frac{\sigma_{i n}^{2}}{\mu_{i n}}=1$, to a compound Poisson case (high variance case), e.g., $\frac{\sigma_{i n}^{2}}{\mu_{i n}} \geq 1$. In experimental analysis, the variance-to-mean ratio for the total demand has a critical importance.

Furthermore, let $M_{n}=\sum_{i \in I} \mu_{i n}$ denote the average total demand rate per unit time for warehouse $n \in N \cup\{0\}$. Then, by using Little's law, the aggregate mean response time at warehouse $n \in N$ can be reformulated for the compound Poisson demand case as

$$
\begin{aligned}
W_{n}(\vec{Q}, \vec{R}, \vec{S}) & =\sum_{i \in I} \frac{\mu_{i n}}{M_{n}} E\left[W_{i n}\left(Q_{i}, R_{i}, S_{i n}\right)\right]=\sum_{i \in I} \frac{\mu_{i n}}{M_{n}} \frac{E\left[B_{i n}\left(Q_{i}, R_{i}, S_{i n}\right)\right]}{\mu_{i n}} \\
& =\sum_{i \in I} \frac{E\left[B_{i n}\left(Q_{i}, R_{i}, S_{i n}\right)\right]}{M_{n}} .
\end{aligned}
$$

Similarly, we have $W_{0}(\vec{Q}, \vec{R})=\sum_{i \in I} \frac{E\left[B_{i 0}\left(Q_{i}, R_{i}\right)\right]}{M_{0}}$. Then the problem for compound Poisson demand $\left(P_{c}\right)$ is formulated as

$$
\begin{aligned}
& \operatorname{Min} \sum_{i \in I}\left[c_{i} h\left(E\left[I_{i 0}\left(Q_{i}, R_{i}\right)\right]+\sum_{n \in N} E\left[I_{i n}\left(Q_{i}, R_{i}, S_{i n}\right)\right]\right)+\frac{\mu_{i 0} K_{i}}{Q_{i}}\right] \\
& \text { s.t. } \quad \sum_{i \in I} \frac{E\left[B_{i 0}\left(Q_{i}, R_{i}\right)\right]}{M_{0}} \leq W_{0}^{\max } \\
& \sum_{i \in I} \frac{E\left[B_{i n}\left(Q_{i}, R_{i}, S_{i n}\right)\right]}{M_{n}} \leq W_{n}^{\max }, \quad \text { for } \forall n \in N, \\
& Q_{i} \geq 1, R_{i} \geq-1, S_{i n} \geq 0, \text { and } Q_{i}, R_{i}, S_{\text {in }} \in Z, \quad \text { for } \forall i \in I, \forall n \in N \text {. }
\end{aligned}
$$

where the objective function (5.1) minimizes the system-wide inventory holding and fixed ordering costs, while constraint (5.2) and (5.3) guarantee that aggregate mean response times at the central and local warehouses do not exceed $W_{0}^{\max }$ and $W_{n}^{\max }$, respectively. 
Table 5.1: General Notation.

\begin{tabular}{|c|c|}
\hline$i$ & Part index, $i \in I$ \\
\hline$n$ & Warehouse index $n \in N \cup\{0\}$ \\
\hline$c_{i}$ & Unit variable cost of part $i$ \\
\hline$h$ & Inventory carrying charge \\
\hline$K_{i}$ & Fixed ordering cost of part $i$ at the central warehouse \\
\hline$\lambda_{\text {in }}$ & Customer arrival rate for part $i$ at local warehouse $n \in N$ \\
\hline$\lambda_{i 0}^{e}$ & External customer arrival rate for part $i$ at the central warehouse \\
\hline$\lambda_{i 0}$ & Customer arrival rate (sum of internal and external) for part $i$ at the central warehouse \\
\hline$\mu_{i n}$ & Demand rate for part $i$ at local warehouse $n \in N$ \\
\hline$\mu_{i 0}^{e}$ & External demand rate for part $i$ at the central warehouse \\
\hline$\mu_{i 0}$ & Demand rate (sum of internal and external) for part $i$ at the central warehouse \\
\hline$\sigma_{i n}^{2}$ & Demand variance for part $i$ at local warehouse $n \in N \cup\{0\}$ \\
\hline$M_{n}^{e}$ & Total external demand rate at the central warehouse \\
\hline$M_{n}$ & Total demand rate for warehouse $n \in N \cup\{0\}$ \\
\hline$T_{i 0}$ & Lead time for part $i$ at the central warehouse from the outside supplier \\
\hline$T_{\text {in }}$ & Transportation lead time $T_{i n}$ from the central warehouse to local warehouse $n \in N$ for part $i$ \\
\hline$Y_{\text {in }}$ & Number of customer arrivals during lead time $T_{i n}$ at warehouse $n \in N \cup\{0\}$ for part $i$ \\
\hline$V_{\text {in }}$ & Demand size for part $i$ at warehouse $n \in N \cup\{0\}$ \\
\hline$D_{\text {in }}$ & Demand during lead time $T_{i n}$ at warehouse $n \in N \cup\{0\}$ for part $i$ \\
\hline$Y_{i 0}^{e}$ & Number of external customer arrivals during lead time $T_{i 0}$ at the central warehouse for part $i$ \\
\hline$V_{i 0}^{e}$ & External demand size for part $i$ at the central warehouse \\
\hline$D_{i 0}^{e}$ & External demand during lead time $T_{i 0}$ at the central warehouse for part $i$ \\
\hline$v_{i n}^{k}$ & Size of $k^{\text {th }}$ demand occurred during the lead time $T_{\text {in }}$ at warehouse $n \in N \cup\{0\}$ for part $i$ \\
\hline$W_{n}^{\max }$ & Target aggregate mean response time at warehouse $n \in N \cup\{0\}$ \\
\hline$R_{i}$ & Reorder level for part $i$ at the central warehouse (decision variable) \\
\hline$Q_{i}$ & Order quantity for part $i$ at the central warehouse (decision variable) \\
\hline$S_{\text {in }}$ & Base-stock level for part $i$ at local warehouse $n \in N$ (decision variable) \\
\hline$\vec{S}_{i}$ & {$\left[S_{i 1}, S_{i 2}, \ldots, S_{i|N|}\right]=$ Vector of base-stock levels for part $i$} \\
\hline$\vec{S}$ & {$\left[\vec{S}_{1}, \vec{S}_{2}, \ldots, \vec{S}_{|I|}\right]=$ Vector of base-stock levels } \\
\hline$\vec{Q}$ & {$\left[Q_{1}, Q_{2}, \ldots, Q_{\mid I]}\right]=$ Vector of order quantities } \\
\hline$\vec{R}$ & {$\left[R_{1}, R_{2}, \ldots, R_{\mid I]}\right]=$ Vector of reorder levels } \\
\hline$I_{\text {in }}\left(Q_{i}, R_{i}, S_{\text {in }}\right)$ & On-hand inventory level for part $i$ at warehouse $n \in N$ in the steady state \\
\hline$I_{i 0}\left(Q_{i}, R_{i}\right)$ & On-hand inventory level for part $i$ at the central warehouse in the steady state \\
\hline$X_{\text {in }}\left(Q_{i}, R_{i}\right)$ & Number of outstanding orders for part $i$ at warehouse $n \in N$ in the steady state \\
\hline$B_{\text {in }}\left(Q_{i}, R_{i}, S_{\text {in }}\right)$ & Backorder level for part $i$ at warehouse $n \in N$ in the steady state \\
\hline$B_{i 0}\left(Q_{i}, R_{i}\right)$ & Backorder level for part $i$ at the central warehouse in the steady state \\
\hline$B_{i 0}^{(n)}\left(Q_{i}, R_{i}\right)$ & Backorder level of local warehouse $n$ for part $i$ at the central warehouse in the steady state \\
\hline$W_{\text {in }}\left(Q_{i}, R_{i}, S_{\text {in }}\right)$ & Response time for part $i$ at warehouse $n \in N$ in the steady state \\
\hline$W_{i 0}\left(Q_{i}, R_{i}\right)$ & Response time for part $i$ at the central warehouse in the steady state \\
\hline$W_{i 0}^{e}\left(Q_{i}, R_{i}\right)$ & Response time for part $i$ at the central warehouse (based on external customers) \\
\hline$W_{n}(\vec{Q}, \vec{R}, \vec{S})$ & Aggregate mean response time at warehouse $n \in N$ in the steady state \\
\hline$W_{0}(\vec{Q}, \vec{R})$ & Aggregate mean response time at the central warehouse in the steady state \\
\hline$W_{0}^{e}(\vec{Q}, \vec{R})$ & Aggregate mean response time at the central warehouse (based on external customers) \\
\hline
\end{tabular}


As in the Poisson demand model, one could also model the problem by considering only the external customers to evaluate the performance of the central warehouse. Then, the aggregate mean response time at the central warehouse is stated by $W_{0}^{e}(\vec{Q}, \vec{R})=\sum_{i \in I} \frac{\mu_{i 0}^{e}}{M_{0}^{e}} E\left[W_{i 0}^{e}\left(Q_{i}, R_{i}\right)\right]$, where $\mu_{i 0}^{e}=\mu_{i 0}-\sum_{n \in N} \mu_{i n}$ and $M_{0}^{e}=M_{0}-\sum_{n \in N} M_{n}$ are the corresponding demand rates associated with the external demands at the central warehouse. Since there is no differentiation between the external and the internal demands we simply have $W_{i 0}^{e}(\vec{Q}, \vec{R})=W_{i 0}(\vec{Q}, \vec{R})$. Then, we obtain

$$
W_{0}^{e}(\vec{Q}, \vec{R})=\sum_{i \in I} \frac{\mu_{i 0}^{e}}{M_{0}^{e}} E\left[W_{i 0}\left(Q_{i}, R_{i}\right)\right]=\sum_{i \in I} \frac{\mu_{i 0}^{e}}{M_{0}^{e}} \frac{E\left[B_{i 0}\left(Q_{i}, R_{i}\right)\right]}{\mu_{i 0}},
$$

which replaces constraint (5.2).

In the rest of this section, first, we obtain the distribution of $D_{\text {in }}$ for each $i \in I$ and $n \in N \cup\{0\}$. Then, we introduce the exact and the approximate methods considered to evaluate the-steady state performance of the system.

\subsubsection{Obtaining the Lead Time Demand Distributions}

For each $i \in I$ and $n \in N$, the distribution of the external demand during lead time of $T_{i n}$ is obtained by using $D_{i n}=\sum^{Y_{i n}} V_{i n}$ and the distributions of $Y_{i n}$ and $V_{i n}$, where $Y_{i n}$ has a Poisson distribution with customer arrival rate $\lambda_{i n}$ and $V_{i n}$ has a compounding distribution denoted by $P\left(V_{i n}=v\right)$. Without loss of generality, the demand sizes are assumed to be non-zero, i.e., $P\left(V_{\text {in }}=0\right)=0$. Let $P\left(\mathcal{V}_{i n}^{k}=v\right)$ give the $v$-fold convolution of $P\left(V_{\text {in }}=v\right)$, i.e., the probability that $k$ customers yields a total demand of $v$. Then, $P\left(\mathcal{V}_{i n}^{k}=v\right)$ is obtained recursively by using

$$
P\left(\mathcal{V}_{i n}^{k}=v\right)=\sum_{i=k-1}^{v-1} P\left(\mathcal{V}_{i n}^{k-1}=i\right) P\left(V_{i n}=v-i\right),
$$

for each $k \geq 2, n \in N$ and $i \in I$. Finally, the distribution of $D_{i n}$ is determined by using

$$
P\left(D_{\text {in }}=d\right)=\sum_{x=1}^{d} P\left(Y_{\text {in }}=x\right) P\left(\mathcal{V}_{\text {in }}^{x}=d\right),
$$

where $P\left(\mathcal{V}_{\text {in }}^{1}=v\right)=P\left(V_{\text {in }}=v\right)$ and $P\left(\mathcal{V}_{\text {in }}^{0}=v\right)=0$ for each $n \in N$ and $i \in I$. 
In a similar way, the distribution of the external demand during lead time $T_{i 0}$ for part $i \in I$ at the central warehouse, $D_{i 0}^{e}$, is determined by using the distributions of $Y_{i 0}^{e}$ and $V_{i n}^{e}$, where $Y_{i 0}^{e}$ has a Poisson distribution with customer arrival rate $\lambda_{i n}^{e}$ and $V_{i n}^{e}$ has a compounding distribution denoted by $P\left(V_{i n}^{e}=v\right)$. Since for each part $i \in I, D_{i n}$ for all $n \in N$ and $D_{i 0}^{e}$ are independent compound Poisson random variables with customer arrival rate $\lambda_{i n}$ and $\lambda_{i n}^{e}$ and compounding distributions $P\left(V_{\text {in }}=v\right)$ and $P\left(V_{i n}^{e}=v\right)$, respectively, $D_{i 0}$ has also a compound Poisson distribution with customer

arrival rate $\lambda_{i 0}=\lambda_{i 0}^{e}+\sum_{n \in N} \lambda_{i n}$ and compounding distribution $P\left(V_{i 0}=v\right)=\frac{\lambda_{i 0}^{e}}{\lambda_{i 0}} P\left(V_{i 0}^{e}=\right.$ $v)+\sum_{n \in N} \frac{\lambda_{i n}}{\lambda_{i 0}} P\left(V_{i n}=v\right)\left(\right.$ Kaas et al. 2001). Therefore, the distribution of $D_{i 0}$ is obtained in a similar way.

\subsection{An Exact Evaluation Based on a Flow-Unit Method}

We adopt the exact evaluation method proposed by Axsäter (2000) for the exact evaluation of our system. The method relies on the flow-unit method widely used in the literature (Forsberg 1995, Axsäter 1995, Axsäter and Zhang 1996, Axsäter 1998). Axsäter's method is originally developed for a two-echelon inventory distribution system in which both the central warehouse and the local warehouses operate under a $(Q, R)$ policy. Note that for a given part $i \in I$, our system is a special case of this system. At each local warehouse $n \in N$, the order quantities are multiples of 1 and the reorder level is $S_{i n}-1$, i.e., whenever the inventory position of local warehouse $n \in N$ drops to or below reorder level $S_{\text {in }}-1$, a sufficient number of orders with batch size one are placed so that the inventory position exceeds $S_{i n}-1$. At the central warehouse, the order quantities are multiples of $Q_{i}$ and the reorder level is $R_{i}$, i.e., whenever the inventory position of the central warehouse drops to or below reorder level $R_{i}$, a sufficient number of orders with batch size $Q_{i}$ are placed so that the inventory position exceeds $R_{i}$. Therefore, Axsäter's method is adopted to our system by setting the batch size (based on the definition of Axsäter 2000) and inventory position for each part $i \in I$ at each local warehouse $n \in N$ to 1 and $S_{i n}$, respectively. After using this method, we obtain the steady-state probabilities for $I_{i n}$ and $B_{i n}$. Then, by using these probabilities, we calculate $E\left[I_{i n}\right]$ in (5.1) and $E\left[B_{i n}\right]$ in (5.3) for each $i \in I$ and $n \in N$. Finally, it remains to obtain $E\left[I_{i 0}\right]$ and $E\left[B_{i 0}\right]$ for each $i \in I$. 
For compound Poisson demand, under a base-stock policy, since every demand triggers an order, the inventory position is constant at the base-stock level. Hence, equation (3.6) holds for the compound Poisson model as well. Therefore, we simply have

$$
S_{\text {in }}=I_{\text {in }}-B_{\text {in }}+X_{\text {in }} \text {. }
$$

Similarly, since for each part $i \in I$ the number of outstanding orders at time $t$ at local warehouse $n \in N$ equals to the sum of the number of backorders dedicated to local warehouse $n \in N$ at the central warehouse at $t-T_{\text {in }}$ and the demand during lead time $T_{i n}$, we have

$$
X_{i n}(t)=B_{i 0}^{(n)}\left(t-T_{i n}\right)+D_{i n}\left(t-T_{i n}, t\right)
$$

or simply $X_{i n}=B_{i 0}^{(n)}+D_{i n}$. Furthermore, since customer orders at the central warehouse are not differentiated, the average waiting time at the central warehouse is the same for all parts no matter from which warehouse the part comes from. Therefore, for any part $i$, average waiting time of orders emanating from local warehouse $n$ at the central warehouse, $E\left[W_{i 0}^{(n)}\right]$, is given as $E\left[W_{i 0}^{(n)}\right]=E\left[W_{i 0}\right]$, or equivalently $\frac{E\left[B_{i 0}^{(n)}\right]}{\mu_{i n}}=\frac{E\left[B_{i 0}\right]}{\mu_{i 0}}$. Therefore, we have

$$
E\left[B_{i 0}^{(n)}\right]=\frac{\mu_{i n}}{\mu_{i 0}} E\left[B_{i 0}\right]
$$

Note that this result is valid also for the external customers at the central warehouse. Based on these results, we obtain $E\left[I_{i 0}\right]$ in (5.1) and $E\left[B_{i 0}\right]$ in (5.2) for each $i \in I$ as follows: First, from (5.6), we have $E\left[B_{i 0}\right]=\frac{\mu i 0}{\mu_{i n}} E\left[B_{i 0}^{(n)}\right]$. Then, by using (5.5), we obtain $E\left[B_{i 0}\right]=\frac{\mu_{i 0}}{\mu_{i n}}\left(E\left[X_{i n}\right]-E\left[D_{i n}\right]\right)$. Furthermore, by using (5.4), we establish $E\left[B_{i 0}\right]=\frac{\mu_{i 0}}{\mu_{i n}}\left(E\left[B_{i n}\right]+S_{i n}-E\left[I_{i n}\right]-E\left[D_{i n}\right]\right)$. Finally, one can obtain $E\left[I_{i 0}\right]$ easily by using $E\left[B_{i 0}\right]$, see (5.10). Therefore, in the flow-unit method, after finding $E\left[I_{i n}\right]$ and $E\left[B_{i n}\right]$ one can obtain $E\left[I_{i 0}\right]$ and $E\left[B_{i 0}\right]$ directly by using $E\left[I_{i n}\right]$ and $E\left[B_{i n}\right]$. Nevertheless, the method is intractable for large problems since it requires too many expressions and convolutions to be computed (Axsäter 2000). 


\subsection{Approximate Evaluation Methods Based on a Disaggregation Method}

In this section, we introduce four alternative approximations for the evaluation of the steady-state behaviour of our system. Although approximate evaluation methods do not guarantee feasibility when they are employed in a policy optimization problem, it is necessary to solve practical size problems under a compound Poisson setting. The first method relies on the disaggregation method (or so called the lead-time demand method) that we use for the Poisson demand setting in Chapter 3. The other three approximations can be derived from the first one. Therefore, we start with introducing the first method, and then explain the other three approximations based on the first one.

For the first approximation, we follow exactly the same the procedure in Section 3.1. That is, first, we find the steady-state probability distributions of the inventory and the backorder levels at the central warehouse by using

$$
\begin{gathered}
P\left\{I_{i 0}\left(Q_{i}, R_{i}\right)=x\right\}= \begin{cases}\frac{1}{Q_{i}} \sum_{k=\max \left(R_{i}+1, x\right)}^{R_{i}+Q_{i}} P\left\{D_{i 0}=k-x\right\}, & \text { for } 1 \leq x \leq R_{i}+Q_{i}, \\
\frac{1}{Q_{i}} \sum_{k=R_{i}+1}^{R_{i}+Q_{i}} P\left\{D_{i 0} \geq k\right\}, & \text { for } x=0,\end{cases} \\
P\left\{B_{i 0}\left(Q_{i}, R_{i}\right)=x\right\}= \begin{cases}\frac{1}{Q_{i}} \sum_{k=R_{i}+1}^{R_{i}+Q_{i}} P\left\{D_{i 0}=k+x\right\}, & \text { for } x \geq 1, \\
\frac{1}{Q_{i}} \sum_{k=R_{i}+1}^{R_{i}+Q_{i}} P\left\{D_{i 0} \leq k\right\}, & \text { for } x=0,\end{cases}
\end{gathered}
$$

Then, we obtain the steady-state probability distribution of $P\left\{B_{i 0}^{(n)}\left(Q_{i}, R_{i}\right)\right\}$ by using (3.8) as in the Poisson demand case. Recall that in the Poisson demand setting, $B_{i 0}^{(n)} \mid B_{i 0}$ is binomially distributed with parameters $B_{i 0}$ and $\frac{\lambda_{i n}}{\lambda_{i 0}}$, which is also known as binomial disaggregation in the literature. Although this holds for the Poisson demand setting, it is not true for the compound Poisson demand case. Assuming that $B_{i 0}^{(n)} \mid B_{i 0}$ is binomially distributed with parameters $B_{i 0}$ and $\frac{\mu_{i n}}{\mu_{i 0}}$ (or equivalently one may consider also $\frac{\lambda_{i n}}{\lambda_{i 0}}$ ) and then using (3.8) to obtain $B_{i 0}^{(n)}$ is our first approximation. We simply call this method "binomial approximation". By using this approximation and (5.5) the steady-state probability distribution of $X_{i n}\left(Q_{i}, R_{i}\right)$ is obtained as follows: 


$$
P\left\{X_{\text {in }}\left(Q_{i}, R_{i}\right)=x\right\}=\sum_{y=0}^{x} P\left\{D_{\text {in }}=y\right\} \cdot P\left\{B_{i 0}^{(n)}\left(Q_{i}, R_{i}\right)=x-y\right\}, \text { for } x \geq 0 .
$$

Then, the steady-state probability distribution of $I_{\text {in }}\left(Q_{i}, R_{i}, S_{\text {in }}\right)$ is obtained from (3.9) as in the Poisson demand case. By using the distributions of inventory levels at the warehouses, the expected inventory levels $E\left[I_{i 0}\right]$ and $E\left[I_{i n}\right]$ for each $n \in N$ in the objective function (5.1) are derived. Finally, the expected backorder expressions in constraints (5.2) and (5.3) are obtained from

$$
\begin{aligned}
& E\left[B_{i 0}\right]=E\left[D_{i 0}\right]-R_{i}-\frac{\left(Q_{i}+1\right)}{2}+E\left[I_{i 0}\right], \\
& E\left[B_{i n}\right]=E\left[X_{i n}\right]-S_{i n}+E\left[I_{i n}\right] .
\end{aligned}
$$

Recall that under the compound Poisson demand, the inventory position at the central warehouse may overshoot the reorder level $R_{i}$ since demand sizes are random. Gallego et al. (2007) propose a two-moment approximation for a single-item two-echelon distribution system operating under a base-stock policy. It is based on distributing the overshoot quantity among the local warehouses in the same way as the demand. The method is also considered by Özer and Xiong (2008) for approximating a single-item two-echelon distribution system with service-level constraints. It yields satisfactory results and offers a better performance when the compounding distributions at the local warehouses are similar and the overshoot is a small part of the backorder level $B_{i 0}$ at the central warehouse, which corresponds to the case with low demand variance (Gallego et al. 2007, Özer and Xiong 2008). As a second approximation, we extend this method to multi-item batch ordering systems and instead of using (3.8) to obtain the distribution of $B_{i 0}^{(n)}$, we approximate it by using a two-moment approximation in Gallego et al. (2007). Based on this method, $E\left[B_{i 0}^{(n)}\right]$ is obtained from (5.6), while $\operatorname{Var}\left(B_{i 0}^{(n)}\right)$ is obtained from

$$
\operatorname{Var}\left(B_{i 0}^{(n)}\right)=\left(\frac{\mu_{i n}}{\mu_{i 0}}\right)^{2} \operatorname{Var}\left(B_{i 0}\right)+\left(\frac{\lambda_{i n}}{\lambda_{i 0}} E\left[V_{i n}^{2}\right]-\left(\frac{\mu_{i n}}{\mu_{i 0}}\right)^{2} E\left[V_{i 0}^{2}\right]\right) \frac{E\left[B_{i 0}\right]}{E\left[V_{i 0}\right]}
$$

We call this second approximation "two-moment approximation for the distribution of backorders at the central warehouse emanating from local warehouses", or simply "two-moment approximation for backorders". 
As a third approximation, instead of using (3.8) and then (5.9) to obtain the distribution of $X_{i n}$, we directly approximate it by using a two-moment approximation similar to the one described in Graves (1985). The method is widely used in evaluating the two-echelon inventory control systems under a base-stock policy (Caggiano et al. 2007, Özer and Xiong 2008) and also the systems under a batch ordering policy (Graves 1985, Hopp et al. 1999). Furthermore, it outperforms METRIC under singleitem (Graves 1985) and multi-item settings (Wong et al. 2007b). Note that Graves' method is originally developed for the pure Poisson case in which it is relatively easy to obtain $\operatorname{Var}\left(B_{i 0}^{(n)}\right)$ since $B_{i 0}^{(n)} \mid B_{i 0}$ is binomially distributed with parameters $B_{i 0}$ and $\frac{\lambda_{i n}}{\lambda_{i 0}}$. Nevertheless, finding $\operatorname{Var}\left(X_{i n}\right)$ is quite involved in the compound Poisson demand setting. Several papers in the literature extend this method to a compound setting (Gallego et al. 2007, Özer and Xiong 2008, Zipkin 2000). As opposed these papers, here, we extend Graves' two-moment approximation to a batch ordering system under a compound Poisson setting by using the results in Gallego et al. 2007 and Özer and Xiong (2008). Accordingly, we obtain the relevant moments from

$$
\begin{aligned}
E\left[X_{i n}\right] & =E\left[B_{i 0}^{(n)}\right]+E\left[D_{i n}\right], \\
\operatorname{Var}\left(X_{i n}\right) & =\operatorname{Var}\left(B_{i 0}^{(n)}\right)+\operatorname{Var}\left(D_{i n}\right),
\end{aligned}
$$

which are obtained from (5.5), while $E\left[B_{i 0}^{(n)}\right]$ and $\operatorname{Var}\left(B_{i 0}^{(n)}\right)$ are obtained from (5.6) and (5.12), respectively. In this approximation, although (5.5) is exact, $\operatorname{Var}\left(X_{i n}\right)$ is approximate because $\operatorname{Var}\left(B_{i 0}^{(n)}\right)$, which is obtained from equation (5.12), is not exact. Therefore, our method involves an additional approximation compared to the original method (Graves 1985), in which the first and second moments are exact. Later, in the computational study we see that this has some negative effect on the performance of the approximation in comparison to the results of Graves' method under Poisson demand settings (Graves 1985, Wong et al. 2007b). We call this third approximation "two-moment approximation for outstanding orders".

Finally, we also adapt the METRIC approximation (Sherbrooke 1968) to our problem. The method relies on replacing the replenishment lead time at the local warehouse $n \in N$ for each part $i \in I$ by its mean, e.g., $T_{i n}+E\left[W_{i 0}\right]$. Since the stochastic lead time is replaced by a constant, it simplifies the evaluation of the system (Axsäter 2000). 
Therefore, the method is quite common in the literature. However it is also known that the METRIC underestimate the correct lead time. Therefore results in understocking and, hence, high level of infeasibility (Wong et al. 2007b, Graves 1985).

\subsection{Solution Procedures}

In this section, we extend the heuristics and the lower bound proposed for the Poisson demand case to compound Poisson demand setting. The column generation method, greedy algorithm and the sequential heuristics are directly applicable to problem $P_{c}$. The column generation method introduced for the Poisson demand model in Section 3.1 requires the following minor changes:

- In the master problem $M P, A_{i l 0}$ and $A_{i l n}$ are redefined as $A_{i l 0}=\frac{E\left[B_{i 0}\left(Q_{i}^{l}, R_{i}^{l}\right)\right]}{M_{0}}$ and $A_{i l n}=\frac{E\left[B_{i n}\left(Q_{i}^{l}, R_{i}^{l}, S_{i n}^{l}\right)\right]}{M_{n}}$ for each column $l \in L$, for part $i \in I$ and for each warehouse $n \in N$.

- In the pricing problem $(C G), A_{i 0}$ and $A_{i n}$ are redefined as $A_{i 0}=\frac{E\left[B_{i 0}\left(Q_{i}, R_{i}\right)\right]}{M_{0}}$ and $A_{\text {in }}=\frac{E\left[B_{i n}\left(Q_{i}, R_{i}, S_{i n}\right)\right]}{M_{n}}$ for each part $i \in I$ and for warehouse $n \in N$.

- In subproblem $S P_{i}(\vec{\theta}), \theta_{n}$ is redefined as $\theta_{n}=\frac{-\alpha_{n}}{M_{n}}$ for each $n \in N \cup\{0\}$ and for each part $i \in I$.

After making these changes, the column generation procedure is implemented in a similar way to obtain the Lagrangian dual solution for problem $P_{c}$. Hence, in an iterative procedure $C G$ is solved to obtain the columns required for the solution of $L P$ relaxation of $M P$, i.e., $\left(Q_{i}^{l}, R_{i}^{l}, \vec{S}_{i}^{l}\right)$ for each $i \in I$, the $L P$-relaxation of $M P$ is solved to obtain the dual variables required for the solution of $C G$, i.e., $\alpha_{n}$ for each $n \in N \cup\{0\}$ and $\beta_{i}$ for each $i \in I$. Similarly, to solve problem $C G$, we solve subproblems $\operatorname{SP}_{i}(\vec{\theta})$ for all $i \in I$. The procedure is repeated until none of the subproblems $S P_{i}(\vec{\theta})$ yields a negative optimal objective function value.

To solve each subproblem $S P_{i}(\vec{\theta})$, we adapt the algorithm proposed for solving singleitem two-echelon batching problems with Poisson demand in Section 3.2.2 to a compound Poisson setting. This requires extension of the results in Section 3.2.2 to compound Poisson setting. Therefore, we propose upper bounds $Q_{i}^{U B}$ and $R_{i}^{U B}$ and lower 
bounds $Q_{i}^{L B}$ and $R_{i}^{L B}$ for the optimal values for $Q_{i}$ and $R_{i}$ for each part $i \in I$, respectively. We explain how to find the optimal solution for subproblems for given values of reorder level and order quantity. These are necessary for the implementation of the corresponding algorithm. The details are given in Appendix A.

After finding the Lagrangian dual solution, we use the greedy algorithm in Section 3.2.3 to obtain a feasible solution starting from the Lagrangian dual solution. In this way, we develop the Lagrangian heuristic for the compound Poisson model.

In order to implement the sequential heuristics we follow the procedure in Section 2.2. The sequential heuristic requires the following minor changes: The EOQ and the EOQ with planned backorders $\left(E O Q^{B}\right)$ formulae are revised for the compound Poisson model as $Q_{i}=\sqrt{\frac{2 \mu_{i 0} K_{i}}{c_{i} h}}$ and $Q_{i}=\sqrt{\frac{2 \mu_{i 0} K_{i}\left(c_{i} h+p_{i}\right)}{\left(c_{i} h\right) p_{i}}}$, respectively. To implement the third batch size heuristic, we use the upper bound $Q_{i}^{U B}$ and the lower bound $Q_{i}^{L B}$ developed for the solution of the subproblems $S P_{i}(\vec{\theta})$ provided in Appendix A. In this manner, similar to Section 2.2, we obtain three alternative sequential heuristics: $S_{1}$, $S_{2}$ and $S_{3}$.

The Lagrangian dual bound, obtained through the column generation algorithm, is asymptotically tight in the number of parts for the compound Poisson setting as well. This is easy to show since our proof for the Poisson demand model in Section 4.3 is free of the demand distribution. Therefore this result also holds for the compound Poisson demand setting. Similarly, since the column generation method converge (as shown in Chapter 3) all our heuristics guarantee convergence.

\subsection{Computational Study}

In this section, we conduct a computational study to test the performances of the heuristics and the approximations. Our computational study comprises four parts. First, the performance of the Lagrangian heuristic implemented by using the exact evaluation method is tested against the Lagrangian dual bound. Note that since this bound is known to be asymptotically tight in the number of parts, its performance is verified at least for problem instances with large number of parts. In this way, we evaluate the performance of Lagrangian heuristic, which yields quite satisfactory re- 
sults in the pure Poisson demand case, as we show in Chapter 4, and we extend some of the findings in Chapter 4 to compound Poisson demand setting. The experiment also makes it possible to explore the size of the problems that can be solved by using the exact evaluation method. The performance of the Lagrangian heuristic based on the exact method is evaluated in terms of the percentage cost deviation from the Lagrangian dual solution, which is simply denoted by $P C D_{L D}$. Let $z_{L D}$ be the objective function value of the solution obtained by the Lagrangian dual solution when the exact method is used (exact Lagrangian dual bound) and let $z^{L H}$ be the objective function value of the solution obtained by the Lagrangian heuristic when the exact method is used, then $P C D_{L D}$ is computed as follows: $P C D_{L D}=\frac{\left|z^{L H}-z_{L D}\right|}{z_{L D}}$.

Second, the performances of the Lagrangian and the sequential heuristics implemented by using the approximate evaluation methods are tested against the Lagrangian heuristic that is implemented by using the exact evaluation. In this way, we gain insights about the performances of the heuristics and test the accuracy of the approximations at the same time. Since exact evaluation is only possible for small-size problems, we do not perform a computational study to analyze the performance of the sequential heuristics under the exact method although we do that for the Lagrangian heuristic. Hence, we test the performance of the solutions obtained by the three sequential heuristic based on the approximate evaluation methods. The performance of the heuristics under the approximate evaluation methods is evaluated in terms of the percentage cost difference between the corresponding solution and the solution obtained by the Lagrangian heuristic based on the exact method, which is denoted by $P C D_{L H}$. Let $z$ be the objective function value of any solution to be tested, then $P C D_{L H}$ is computed as follows: $P C D_{L H}=\frac{\left|z-z^{L H}\right|}{z^{L H}}$. To obtain $P C D_{L H}$, we compute the correct value of $z$. That is, for all heuristics that are based on approximate evaluation methods, after we obtain a solution by applying the heuristic and the corresponding approximation, then we obtain evaluate the resulting objective function by using the exact method. Hence, while we compute the $P C D_{L H}$ for any solution, we use the exact $z$ value for the corresponding solution. To test the performances of the heuristics that are based on the approximations, we are also interested in the number/percentage of instances that the corresponding method yields a feasible solution. For infeasible solutions, also it makes sense to measure the level of infeasibility. An 
appropriate measure for the level of infeasibility can be the relative distance to feasible region, which is also considered by Wong et al. (2007b). It is defined as follows: Let $X$ be the solution obtained by any one of the heuristics that is obtained by using one of the approximate evaluation methods and let $W_{n}(X)$ is the aggregate mean response time at warehouse $n \in N$ in the steady state for the corresponding solution, then the relative distance to the feasible region, simply $R D F R$, is obtained by $R D F R=\sum_{n \in N \cup\{0\}}\left\{\left(W_{n}(X)-W_{n}^{\max }\right)^{+}\right\} / \sum_{n \in N \cup\{0\}} W_{n}^{\max }$. Note that this expression is similar to the measure of violation of the constraints used in the evolution of the greedy algorithm in Section 3.2.3.

Third, we test the performance of the Lagrangian dual solutions obtained by using the approximate evaluation methods so that we can find a solution to be used as a benchmark solution later in the experiments with large, practical-size problems. Therefore, we compare the performances of the Lagrangian dual solutions that are based on the approximate evaluation methods against the exact Lagrangian dual solution, or simply the exact Lagrangian dual bound for the problem. To do so, we consider the gap between the corresponding solution and the Lagrangian dual bound, which is denoted by $P G A P^{\prime}$. Let $z^{\prime}$ be the objective function value of the Lagrangian dual solution obtained by any approximate evaluation method, then $P G A P^{\prime}$ is computed as follows: $P G A P^{\prime}=\frac{\left|z^{\prime}-z_{L D}\right|}{z_{L D}}$.

Finally, using the results of the previous parts of the computational study, we conduct experiments with larger problems so that we can obtain results for more realistic cases. As a benchmark solution, we use the solution of the Lagrangian heuristic obtained by using the approximation that yields the best performance. We decide on the best approximation based on the results of the experimental study in the second part of the computational analysis. Hence, the performance of the heuristics is evaluated in terms of the percentage cost difference between the solution obtained by the corresponding method and the benchmark solution. This performance measure is denoted by $P C D_{\text {LHapp }}=\frac{\left|z-z_{a p p}\right|}{z_{a p p}}$, where $z_{a p p}$ is the objective function value of the benchmark solution, i.e., the Lagrangian heuristic that is obtained by using the most appropriate approximation. Since the exact evaluation is intractable for large-scale problems, measuring the level of infeasibility is not possible for the larger problems. Hence, in this part, we rely on the $P C D_{L H}$ and also the results of earlier experiments with 
small-size problems in the second part of the experiments. Hence, all our findings are based on the approximate evaluation, i.e., $z$, the objective function value of any solution to be tested, is evaluated under the corresponding approximation. However, even if this is the case, testing the heuristics obtained by using the approximations is still possible to a certain extent and also it makes sense as we see in the following sections.

The performances of the approximations can also be tested by comparing the complete distributions associated with the approximations with the exact distribution. In the dissertation, we exclude the corresponding analysis and consider it as a future work. We suppose that after making such an analysis our findings make more sense.

In Section 5.5.1, we introduce our experimental design. Then, in Section 5.5.2, the performance of the Lagrangian heuristic implemented by using the exact evaluation method is tested against the Lagrangian dual bound. Later, in Section 5.5.3, the performances of the Lagrangian and the sequential heuristics implemented by using the approximate evaluation methods are tested against the Lagrangian heuristic under the exact evaluation. In Section 5.5.4, we test the performance of the Lagrangian dual solutions obtained by using the approximate evaluation methods. Finally, in Section 5.5.5, using the results of the computational study in 5.5.4, we present results from experiments conducted for large-size problems.

\subsubsection{Experimental Design}

In our experiments, we consider the following seven parameters as the experimental factors: (i) number of parts, $|I|$, (ii) number of local warehouses, $|N|$, (iii) customer

arrival rate, $\lambda_{i n}$, (iv) variance-to-mean ratio of the demand distribution, $\frac{\sigma_{i n}^{2}}{\mu_{i n}}$, (v) unit variable costs, $c_{i}$, (vi) fixed ordering costs, $K_{i}$, (vii) target aggregate mean response times at the warehouses, $W_{n}^{\max }$. Since the lead time $T_{i n}$ at warehouse $n \in N \cup\{0\}$ contributes to the model in the form of lead time demand $\lambda_{i n} T_{i n}$, we do not consider it as a distinct factor. In our computational study, we consider three testbeds. The first testbed is used to explore the effects of all parameters, whereas the second testbed is to observe the effects of the number of parts, the number of local warehouses and the variance-to-mean ratio of the demand more deeply, which are the most critical 
parameters that effect the performances of the heuristics and the approximations. In both testbeds, we consider relatively small problems. This makes it possible to implement both the exact and approximate evaluation methods. These testbeds are used in the computational study in Sections 5.5.2-5.5.4. The third testbed is to extend the findings in 5.5.2-5.5.4 to large, practical size problems. This testbed is used in the computational study in Section 5.5.5. For all testbeds, the following considerations are common: The lead time at the central warehouse, $T_{i 0}$, across all parts, the target aggregate mean response times at the warehouses, $W_{n}^{\max }$, across all warehouses, the lead times at the local warehouses, $T_{i n}$, across all parts and local warehouses are assumed to be identical. We assume that the unit variable costs, $c_{i}$, and the fixed ordering costs, $K_{i}$, are nonidentical across all parts, the customer arrival rates, $\lambda_{i n}$, and the variance-to-mean ratio of the demand distribution, $\frac{\sigma_{i n}^{2}}{\mu_{i n}}$ are nonidentical across all parts and warehouses. The fixed ordering cost of each part and the variance-to-mean ratio $\frac{\sigma_{i n}^{2}}{\mu_{i n}}$ of demand distribution of each part at each location are assumed to be random. Each is generated from a uniform distribution. To represent skewnesses of the customer arrival rates and the unit variable costs across parts, i.e., the Pareto principle, we follow the same approach that we consider for the Poisson demand in Section 4.4.1. Recall that in Section 4.4.1, we obtain instances for symmetric and assymetric demand cases. In a similar way, we obtain symmetric and assymetric demand cases for the Poisson demand setting. However, we consider only the asymmetric demand case, since it is more realistic and there is no significant difference between the two cases in the pure Poisson demand setting.

For the first testbed, we consider 2 levels of each parameter, except the $\frac{\sigma_{i n}^{2}}{\mu_{i n}}$ ratio, to avoid explosion of the number of problem instances. To analyze the effect of $\frac{\sigma_{i n}^{2}}{\mu_{i n}}$ ratio more deeply, we consider 3 levels. The first level, i.e., the one generated from $U[1,1.001]$, corresponds to the special case where $\frac{\sigma_{i n}^{2}}{\mu_{i n}}$ is almost 1 and resembles the pure Poisson demand setting the most. The other two levels corresponds to the situations where $\frac{\sigma_{\text {in }}^{2}}{\mu_{\text {in }}}$ is higher. The number of parts is set to 20 and 50, whereas the number of local warehouses is set to 2 and 3 . Table 5.2 summarizes the values of the parameters used in testbed 1. Based on this setting, 5 random problem instances are generated for each of the $192\left(2^{6} \times 3\right)$ different settings, resulting in a total of 960 problem instances. 
Table 5.2: Parameter values used in testbed 1.

\begin{tabular}{|l|c|c|}
\hline \multicolumn{1}{|c|}{ Parameters } & $\begin{array}{c}\text { Number of } \\
\text { Levels }\end{array}$ & Values \\
\hline$I$ & 2 & 20 and 50 \\
$N$ & 2 & 2 and 3 \\
$\lambda_{\text {in }}$ (arrivals/day) & 2 & 0.02 and 0.005 \\
$c_{i}$ (\$/unit) & 2 & 1000 and 5000 \\
$W_{n}^{\max }$ (day) & 2 & 0.1 and 0.9 \\
$K_{i}(\$ /$ order $)$ & 2 & $\mathrm{U}[50 / 3,100 / 3]$ and $\mathrm{U}[50 / 3,100 / 3]$ \\
$\sigma_{i n}^{2} / \mu_{i n}$ & 3 & $\mathrm{U}[1,1.001], \mathrm{U}[1,1.5], \mathrm{U}[1,2]$ \\
\hline
\end{tabular}

As we mention before, we also generate a second testbed to explore the effect of three parameters simultaneously, i.e., the number of parts, the number of warehouses and the variance-to-mean ratio of the demand distribution. We generate the problem instances for the second testbed in a similar way as we generate the first testbed. We consider 10 levels of the number of parts, 2 levels of the number of local warehouses and 3 levels of the variance-to-mean ratio. We consider a single level of each of the remaining parameter. Here, we take an average setting for these parameters. Table 5.3 summarizes the values of the parameters used in testbed 2. Based on this setting, 12 random problem instances are generated for each of the $60(10 \times 2 \times 3)$ different parameters setting, resulting in a total of 720 problem instances.

For the third testbed, through which we aim at extending our analysis to large-scale problem instances, we consider 7 levels of the number of parts and 2 levels of the variance-to-mean ratio. In order to observe the effect of much higher variance-tomean ratios, we also consider a setting in which this ratio is generated from $U[1,4]$. For the rest of the parameters we consider a single level. The values of the parameters used in this third testbed is summarized in Table 5.4. Based on this setting, 5 random problem instances are generated for each of the different parameters setting. Apart from that, using the same testbed we also carry out experiments for problem instances 
Table 5.3: Parameter values used in testbed 2.

\begin{tabular}{|l|c|c|}
\hline \multicolumn{1}{|c|}{ Parameters } & $\begin{array}{c}\text { Number of } \\
\text { Levels }\end{array}$ & Values \\
\hline$I$ & 10 & $10,20,30,40,50,60,70,60,90$ and 100 \\
$N$ & 2 & 2 and 3 \\
$\lambda_{i n}$ (arrivals/day) & 1 & 0.01 \\
$c_{i}$ (\$/unit) & 1 & 3000 \\
$W_{n}^{\max }$ (day) & 1 & 0.3 \\
$K_{i}(\$ /$ order $)$ & 1 & $\mathrm{U}[100 / 3,200 / 3]$ \\
$\sigma_{i n}^{2} / \mu_{i n}$ & 3 & $\mathrm{U}[1,1.001], \mathrm{U}[1,1.5], \mathrm{U}[1,2]$ \\
\hline
\end{tabular}

with 5,000 parts and 12 local warehouses so that we can further see the performances of the heuristics and the approximations for larger problem instances in terms of the number of local warehouses.

The logarithmic and the geometric distributions are empirically shown to be best fitting distributions for spare parts, with having almost the same fitting rates (Eaves 2002). Although the two distributions are very similar distributions (Eaves 2002, Axsäter 2006), in the case of logarithmic distribution, the demand distribution turns out to be a negative binomial distribution. This simplifies the computations. For the sake of simplicity, we use the logarithmic distribution as a compounding distribution in our experiments. However, since all the heuristics and approximations developed in this chapter are free of the compounding distribution, it is also possible to consider other distributions as well. To illustrate the effect of variance-to-mean ratio on the demand distribution, consider the following example. Let the external customer arrival rate for any two parts at the central warehouse be 0.015 and 0.05 per day, i.e., say $\lambda_{1 n}=0.015$ and $\lambda_{2 n}=0.05$. This corresponds to a situation in which demand arrivals occurs in almost every two months and every 20 days, which are very reasonable frequencies for spare parts in practice. Then, for the corresponding values of customer arrival rates, the probability distribution (since we use a logarithmic compounding distribution, this corresponds to a negative binomial distribution), the mean 
Table 5.4: Parameter values used in testbed 3.

\begin{tabular}{|l|c|c|}
\hline \multicolumn{1}{|c|}{ Parameters } & $\begin{array}{c}\text { Number of } \\
\text { Levels }\end{array}$ & Values \\
\hline$I$ & 7 & $50,100,250,500,1000,3000$ and 5000 \\
$N$ & 1 & 6 \\
$\lambda_{\text {in }}$ (arrivals/day) & 1 & 0.015 \\
$c_{i}$ (\$/unit) & 1 & 3000 \\
$W_{n}^{\max }$ (day) & 1 & 0.3 \\
$K_{i}$ (\$/order) & 1 & $\mathrm{U}[50,100]$ \\
$\sigma_{i n}^{2} / \mu_{\text {in }}$ & 3 & $\mathrm{U}[1,2], \mathrm{U}[1,4]$ \\
\hline
\end{tabular}

and the variance of the lead time demand for different values of variance-to-mean ra-

tio, $\frac{\sigma_{\text {in }}^{2}}{\mu_{\text {in }}}$ are shown in Figure 5.1 (lead time at the central warehouse is assumed to be 10 days). Figure 5.1 shows how the tail of the lead time demand probability distribution at the central warehouse extends and the increase of the mean and the variance of the corresponding distribution with the variance-to-mean ratio.

In all our experiments, it is possible to consider the cases in which (1) only external customers, (2) both type of customers are incorporated in evaluating the performance of the central warehouse. Since the experiments do not reveal any significant difference between the results of the two cases in the Poisson demand case (as shown in Chapter 3), we consider only the latter case since it is more realistic. The inventory carrying charge is taken as $25 \%$ annual. The algorithms are coded in $\mathrm{C}++$ and the experiments are run on an Intel $3 \mathrm{GHz}$ processor with 3.5 GB RAM. In the remainder of this section the results of the experiments are presented and discussed.

\subsubsection{Performance of the Lagrangian Heuristic under the exact evaluation}

As we mention before, to test the performance of the heuristics we use testbed 1, whereas to explore the effects of the number of parts, the number of local warehouses 


\begin{tabular}{|c|ccccccc|}
\hline \multicolumn{2}{|c|}{$\begin{array}{c}\text { Probability distribution of the lead time demand under different variance-to-mean ratios } \\
\text { (average demand rate = 0.015) }\end{array}$} \\
\hline $\begin{array}{c}\text { Total demand } \\
\text { size }\end{array}$ & \multicolumn{7}{c|}{ Variance-to-mean ratio } \\
\hline \multirow{2}{*}{0} & $\mathbf{1}$ & $\mathbf{2}$ & $\mathbf{2 . 5}$ & $\mathbf{3}$ & $\mathbf{3 . 5}$ & $\mathbf{4}$ \\
\hline 1 & $86.07 \%$ & $86.07 \%$ & $86.07 \%$ & $86.07 \%$ & $86.07 \%$ & $86.07 \%$ & $86.07 \%$ \\
2 & $12.91 \%$ & $10.61 \%$ & $9.31 \%$ & $8.45 \%$ & $7.83 \%$ & $7.36 \%$ & $6.98 \%$ \\
3 & $0.97 \%$ & $2.42 \%$ & $2.83 \%$ & $2.95 \%$ & $2.97 \%$ & $2.94 \%$ & $2.90 \%$ \\
4 & $0.05 \%$ & $0.64 \%$ & $1.05 \%$ & $1.28 \%$ & $1.41 \%$ & $1.49 \%$ & $1.53 \%$ \\
5 & $0.00 \%$ & $0.18 \%$ & $0.42 \%$ & $0.61 \%$ & $0.74 \%$ & $0.83 \%$ & $0.89 \%$ \\
6 & $0.00 \%$ & $0.05 \%$ & $0.18 \%$ & $0.30 \%$ & $0.41 \%$ & $0.49 \%$ & $0.55 \%$ \\
7 & $0.00 \%$ & $0.02 \%$ & $0.08 \%$ & $0.16 \%$ & $0.23 \%$ & $0.30 \%$ & $0.35 \%$ \\
8 & $0.00 \%$ & $0.00 \%$ & $0.03 \%$ & $0.08 \%$ & $0.14 \%$ & $0.19 \%$ & $0.23 \%$ \\
9 & $0.00 \%$ & $0.00 \%$ & $0.02 \%$ & $0.04 \%$ & $0.08 \%$ & $0.12 \%$ & $0.15 \%$ \\
10 & $0.00 \%$ & $0.00 \%$ & $0.01 \%$ & $0.02 \%$ & $0.05 \%$ & $0.08 \%$ & $0.10 \%$ \\
& $0.00 \%$ & $0.00 \%$ & $0.00 \%$ & $0.01 \%$ & $0.03 \%$ & $0.05 \%$ & $0.07 \%$ \\
\hline Mean & & & & & & & \\
\hline Variance & 0.1500 & 0.1850 & 0.2164 & 0.2456 & 0.2731 & 0.2993 & 0.3246 \\
& 0.1500 & 0.2775 & 0.4328 & 0.6139 & 0.8192 & 1.0477 & 1.2984 \\
\hline
\end{tabular}

\begin{tabular}{|c|ccccccc|}
\hline \multicolumn{2}{|c|}{$\begin{array}{c}\text { Probability distribution of the lead time demand under different variance-to-mean ratios } \\
\text { (average demand rate = 0.05) }\end{array}$} \\
\hline $\begin{array}{c}\text { Total demand } \\
\text { size }\end{array}$ & $\mathbf{1}$ & $\mathbf{1 . 5}$ & $\mathbf{2}$ & $\mathbf{2 . 5}$ & $\mathbf{3}$ & $\mathbf{3 . 5}$ & $\mathbf{4}$ \\
\hline 0 & $60.65 \%$ & $60.65 \%$ & $60.65 \%$ & $60.65 \%$ & $60.65 \%$ & $60.65 \%$ & $60.65 \%$ \\
1 & $30.33 \%$ & $24.93 \%$ & $21.88 \%$ & $19.86 \%$ & $18.40 \%$ & $17.29 \%$ & $16.41 \%$ \\
2 & $7.58 \%$ & $9.28 \%$ & $9.41 \%$ & $9.21 \%$ & $8.93 \%$ & $8.64 \%$ & $8.37 \%$ \\
3 & $1.26 \%$ & $3.33 \%$ & $4.27 \%$ & $4.69 \%$ & $4.87 \%$ & $4.94 \%$ & $4.94 \%$ \\
4 & $0.16 \%$ & $1.18 \%$ & $1.99 \%$ & $2.49 \%$ & $2.80 \%$ & $3.00 \%$ & $3.11 \%$ \\
5 & $0.02 \%$ & $0.41 \%$ & $0.94 \%$ & $1.36 \%$ & $1.67 \%$ & $1.88 \%$ & $2.04 \%$ \\
6 & $0.00 \%$ & $0.14 \%$ & $0.45 \%$ & $0.75 \%$ & $1.01 \%$ & $1.21 \%$ & $1.36 \%$ \\
7 & $0.00 \%$ & $0.05 \%$ & $0.21 \%$ & $0.42 \%$ & $0.62 \%$ & $0.79 \%$ & $0.93 \%$ \\
8 & $0.00 \%$ & $0.02 \%$ & $0.10 \%$ & $0.24 \%$ & $0.39 \%$ & $0.52 \%$ & $0.64 \%$ \\
9 & $0.00 \%$ & $0.01 \%$ & $0.05 \%$ & $0.14 \%$ & $0.24 \%$ & $0.35 \%$ & $0.45 \%$ \\
10 & $0.00 \%$ & $0.00 \%$ & $0.02 \%$ & $0.08 \%$ & $0.15 \%$ & $0.23 \%$ & $0.31 \%$ \\
& & & & & & & \\
\hline M... & 0.5000 & 0.6166 & 0.7213 & 0.8185 & 0.9102 & 0.9978 & 1.0820 \\
\hline Mariance & 0.5000 & 0.9249 & 1.4427 & 2.0463 & 2.7307 & 3.4923 & 4.3281 \\
\hline
\end{tabular}

Figure 5.1: Effect of variance-to-mean ratio on the lead time demand distribution, its mean and variance. 
and the variance-to-mean ratio more deeply, we use testbed 2. We consider the percentage cost difference between the Lagrangian heuristic under the exact evaluation and the Lagrangian dual bound, $P C D_{L D}$, as a performance measure. We also evaluate the performance of the Lagrangian heuristic in terms of the computational requirements based on our experiments conducted by using testbed 1. A summary of the results for testbed 1 is given in Table 5.5, whereas the results for testbed 2 is summarized in Figure 5.2. The CPU time requirements of the Lagrangian heuristic in testbed 1 is presented in Table 5.6

Table 5.5: Effects of parameters on the performance of the Lagrangian heuristic when the exact evaluation method is used.

\begin{tabular}{|cc|cc|}
\hline \multicolumn{2}{|c|}{ Parameters } & \multicolumn{2}{c|}{ Exact } \\
\hline All instances & & $3.472 \%$ & $26.448 \%$ \\
\hline Number of Parts & 20 & $4.738 \%$ & $26.448 \%$ \\
\hline \multirow{2}{*}{ Number of Warehouses } & 50 & $2.206 \%$ & $16.013 \%$ \\
\hline \multirow{2}{*}{ Variance to Mean Ratio } & 3 & $3.363 \%$ & $20.852 \%$ \\
& 4 & $3.582 \%$ & $26.448 \%$ \\
\hline Average Demand Arrival & 0.005 & $3.847 \%$ & $20.852 \%$ \\
Rate (units/day) & 0.02 & $3.098 \%$ & $26.448 \%$ \\
\hline Average Unit Cost & 1000 & $3.023 \%$ & $18.886 \%$ \\
(\$/unit) & 5000 & $3.922 \%$ & $26.448 \%$ \\
\hline Average Fixed Ordering & 25 & $3.847 \%$ & $26.448 \%$ \\
Cost (\$/order) & 75 & $3.097 \%$ & $20.809 \%$ \\
\hline Target Aggregate Mean & 0.1 & $3.457 \%$ & $26.448 \%$ \\
Response Time (day) & 0.9 & $3.487 \%$ & $20.852 \%$ \\
\hline
\end{tabular}

The main findings are as follows:

- As depicted in Table 5.5, both the average and the maximum $P C D_{L D}$ are found 
to be high. However, both improve as the number of parts becomes larger. Figure 5.2 displays that the average $P C D_{L D}$ that the Lagrangian heuristic yields for all 12 random instances in testbed 2 improves with the number of parts. Accordingly, when the number of parts is 100 and the number of local warehouses is 2 , the average $P C D_{L D}$ are found to be $1.32 \%, 0.83 \%$ and $0.50 \%$ for the low, mid and high variance-to-mean ratios (or simply demand variance cases), respectively. Similarly, when the number of parts is 100 and the number of local warehouses is 3 , the average $P C D_{L D}$ are $2.09 \%, 0.93 \%$ and $0.78 \%$ for the low, mid and high demand variance cases, respectively. Both experimental results for testbed 1 and 2 show that the $P C D_{L D}$ that the Lagrangian heuristic yields increases with the number of local warehouses. However, the effect of the number of parts seems to be more strong compared to the that of the number of local warehouses. All these results are in line with our findings for the pure Poisson case in Section 4.4.3.

- Figure 5.2 also shows that the average $P C D_{L D}$ of the Lagrangian heuristic is lower when the demand variance is higher. This reveals that the Lagrangian heuristic, whose performance is tested to be quite well in the pure Poisson (low variance) case, yields much better results in the compound Poisson demand case as the demand variance increases.

- The average and the maximum CPU time required by the Lagrangian heuristic obtained by using the exact evaluation method (also the ones obtained by using alternative approximation methods) are summarized in Table 5.6. In this table and as well as the others in the rest of this chapter, we abbreviate the two-moment approximation for outstanding orders and the two-moment approximation for backorders as two-moment app. (out) and two-moment app. (back), respectively. Table 5.6 displays that average and the maximum CPU time (in sec.) required by the Lagrangian heuristic are quite high under the exact evaluation even when the number of parts is relatively small, such as 20 and 50. However, they are quite low when approximations are used. These results highlight how the exact evaluation procedure becomes highly involved for the compound Poisson demand and also explain why approximations are very necessary to solve problems under the compound Poisson demand such as the 

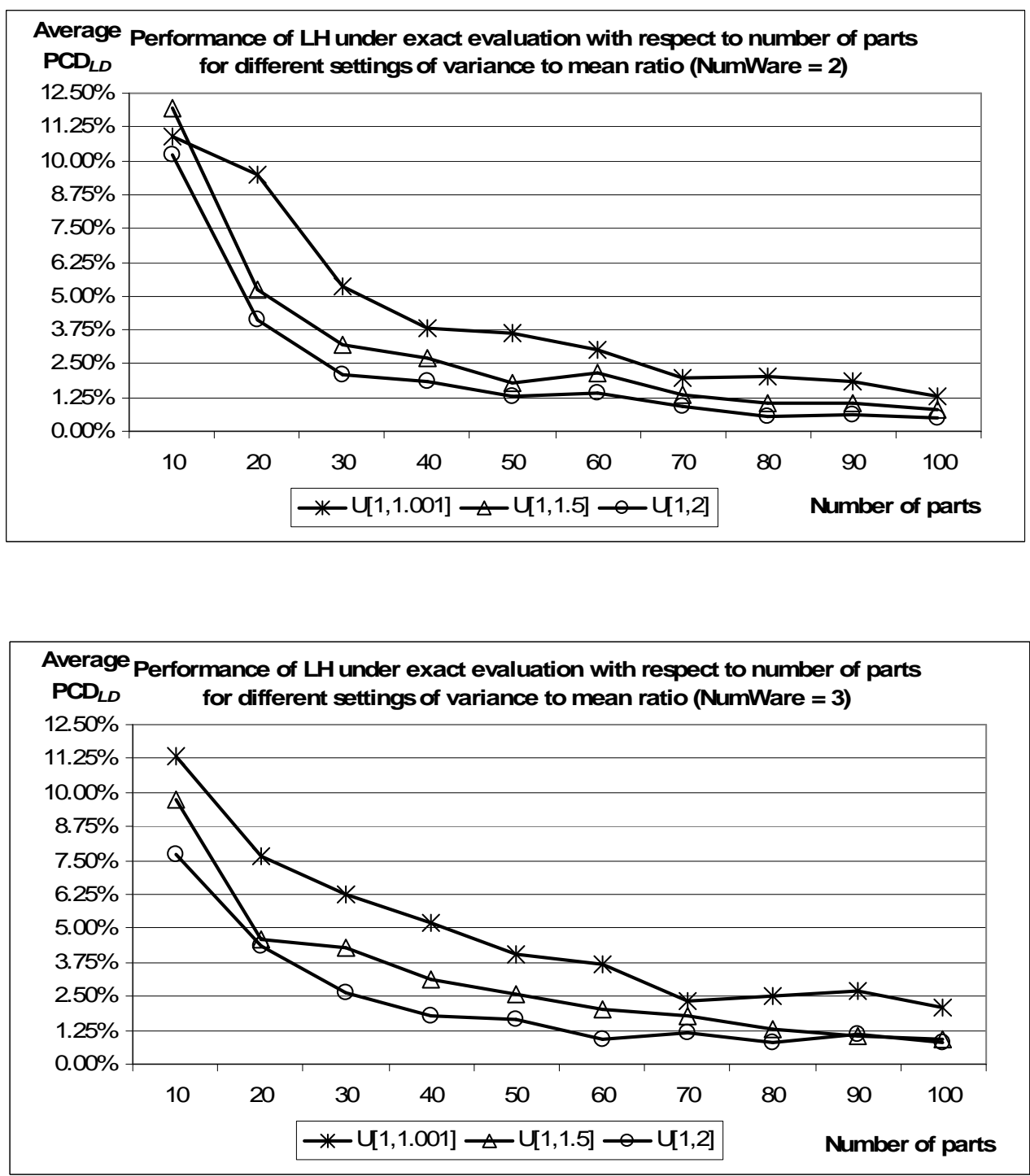

Figure 5.2: Effect of variance-to-mean ratio on the lead time demand distribution, its mean and variance. 
one in this dissertation. Therefore, in the rest of this section, we investigate the performance of the approximations and and try to find out an approximation(s) that can substitute the exact evaluation for larger problems.

Table 5.6: Computational requirements of the Lagrangian heuristics under the exact and approximate evaluation methods (CPU time in sec.)

\begin{tabular}{|c|c|c|c|c|c|c|c|c|c|c|c|}
\hline \multirow{2}{*}{\multicolumn{2}{|c|}{ Parameters }} & \multicolumn{2}{|c|}{ Exact method } & \multicolumn{2}{|c|}{$\begin{array}{c}\text { Binomial } \\
\text { app. }\end{array}$} & \multicolumn{2}{|c|}{ Metric app. } & \multicolumn{2}{|c|}{$\begin{array}{c}\text { Two- } \\
\text { moment } \\
\text { app. (out) }\end{array}$} & \multicolumn{2}{|c|}{$\begin{array}{c}\text { Two- } \\
\text { moment } \\
\text { app. (back) }\end{array}$} \\
\hline & & Avg. & Max & Avg. & Max & Avg. & Max & Avg. & Max & Avg. & Max \\
\hline \multicolumn{2}{|c|}{ All instances (over 960 instances) } & 2732.25 & 125646.28 & 0.23 & 2.00 & 0.06 & 0.23 & 0.06 & 0.25 & 0.10 & 0.55 \\
\hline \multirow{2}{*}{ Number of Parts } & 20 & 1815.96 & 125646.28 & 0.12 & 0.99 & 0.03 & 0.13 & 0.04 & 0.13 & 0.06 & 0.33 \\
\hline & 50 & 3648.55 & 101447.61 & 0.34 & 2.00 & 0.08 & 0.23 & 0.09 & 0.25 & 0.15 & 0.55 \\
\hline \multirow{2}{*}{ Number of Warehouses } & 2 & 1347.11 & 84336.36 & 0.14 & 0.78 & 0.04 & 0.13 & 0.05 & 0.13 & 0.07 & 0.27 \\
\hline & 3 & 4117.40 & 125646.28 & 0.33 & 2.00 & 0.07 & 0.23 & 0.08 & 0.25 & 0.13 & 0.55 \\
\hline
\end{tabular}

\subsubsection{Performance of the heuristics under the approximate evaluation methods}

As mentioned before at the beginning of this section, we consider three different measures to evaluate the performance of the heuristics that are obtained by using the approximate evaluation methods. These are the percentage cost difference between the solution and the exact solution of the Lagrangian heuristic, $P C D_{L H}$, the number of feasible solutions and the relative distance to the feasible region, $R D F R$. Among these three measures, the $P C D_{L H}$ evaluates the performance of the solutions obtained by the heuristic (as well as the approximations) in terms of the objective function values. However, the latter two measures are related with whether the constraints are satisfied, and if not, what level of violation is incurred and how far the solution is from the feasible region.

\subsubsection{The performance of the Lagrangian heuristic under the approximate evaluation methods}

The results for testbed 1 are summarized for each measure separately in Tables 5.75.9. The results for testbed 2 are presented in Figures 5.3 and 5.4, where each point in the figure represents the average and the maximum $P C D_{L H}$ and $R D F R$ for the 12 
problem instances in testbed 2, respectively. In the corresponding figures, we present the results for the settings in which the number of local warehouse is 2 . The results for the settings in which the number of local warehouse is 3 are similar. The main findings based on the $P C D_{L H}$ that the Lagrangian heuristic yields are as follows:

- As depicted in Table 5.7, under each one of the approximations, the average $P C D_{L H}$ that the Lagrangian heuristic yields is quite satisfactory, but the maximum $P C D_{L H}$ is considerably high. However, both the average and the maximum $P C D_{L H}$ that the Lagrangian heuristic yields are quite tolerable in larger, more realistic problem sizes as we discuss later.

- Among four approximate evaluation methods, the two-moment approximations yields better results compared to the binomial approximation and the METRIC. It is well known that the binomial disaggregation fails in the compound Poisson demand setting. Our empirical findings verifies this result and show that the performance of the binomial disaggregation is relatively poor. It is also known that the two-moment approximation yields better results than METRIC in pure Poisson case (Graves 1985, Wong et al. 2007b). Our findings show that this conclusion holds for the compound Poisson demand as well. Finally, the experiments reveal that the two-moment approximation for outstanding orders yields slightly better results than the two-moment approximation for backorders. These results can also be seen in Figure 5.3.

- Although the performance of the Lagrangian heuristic under exact evaluation is found to be better for problems with high demand variance, the result is the opposite when we rely on the approximate evaluation methods. This shows that as the variance-to-mean ratio increases, approximating the outstanding backorders by using any one of the approximations works less. However the effect of demand variance is much more significant for the binomial approximation and performs better when the demand variance is low such as the case in the pure Poisson demand. This can also be seen in Figure 5.3.

- As shown in Table 5.7, under the binomial and the two-moment approximations, the Lagrangian heuristic yields a better performance when the number of parts is higher. Note that this is in line with our findings in the pure Pois- 

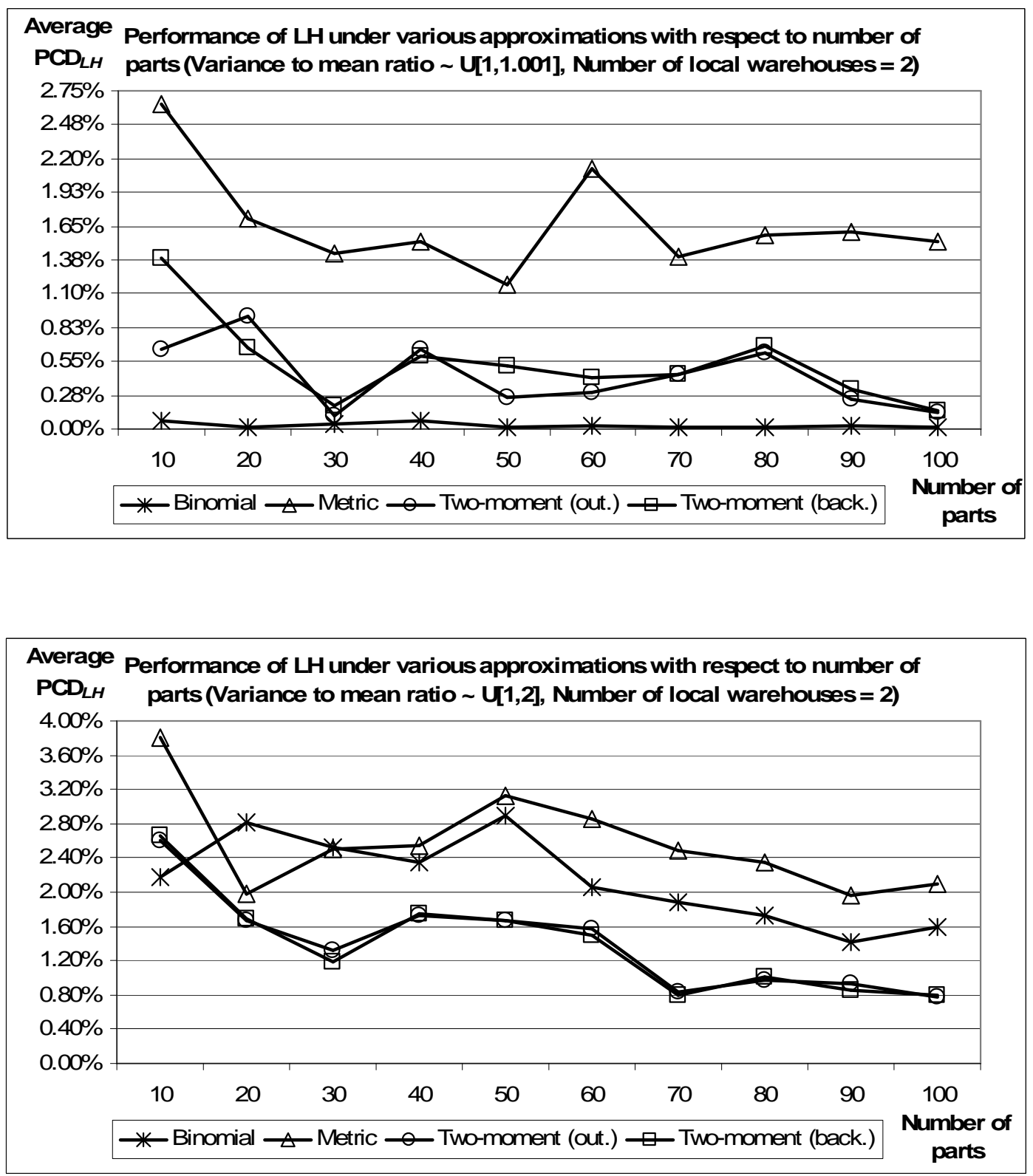

Figure 5.3: Effect of the number of parts on the performance of the Lagrangian heuristic under various approximations $\left(P C D_{L H}\right)$. 

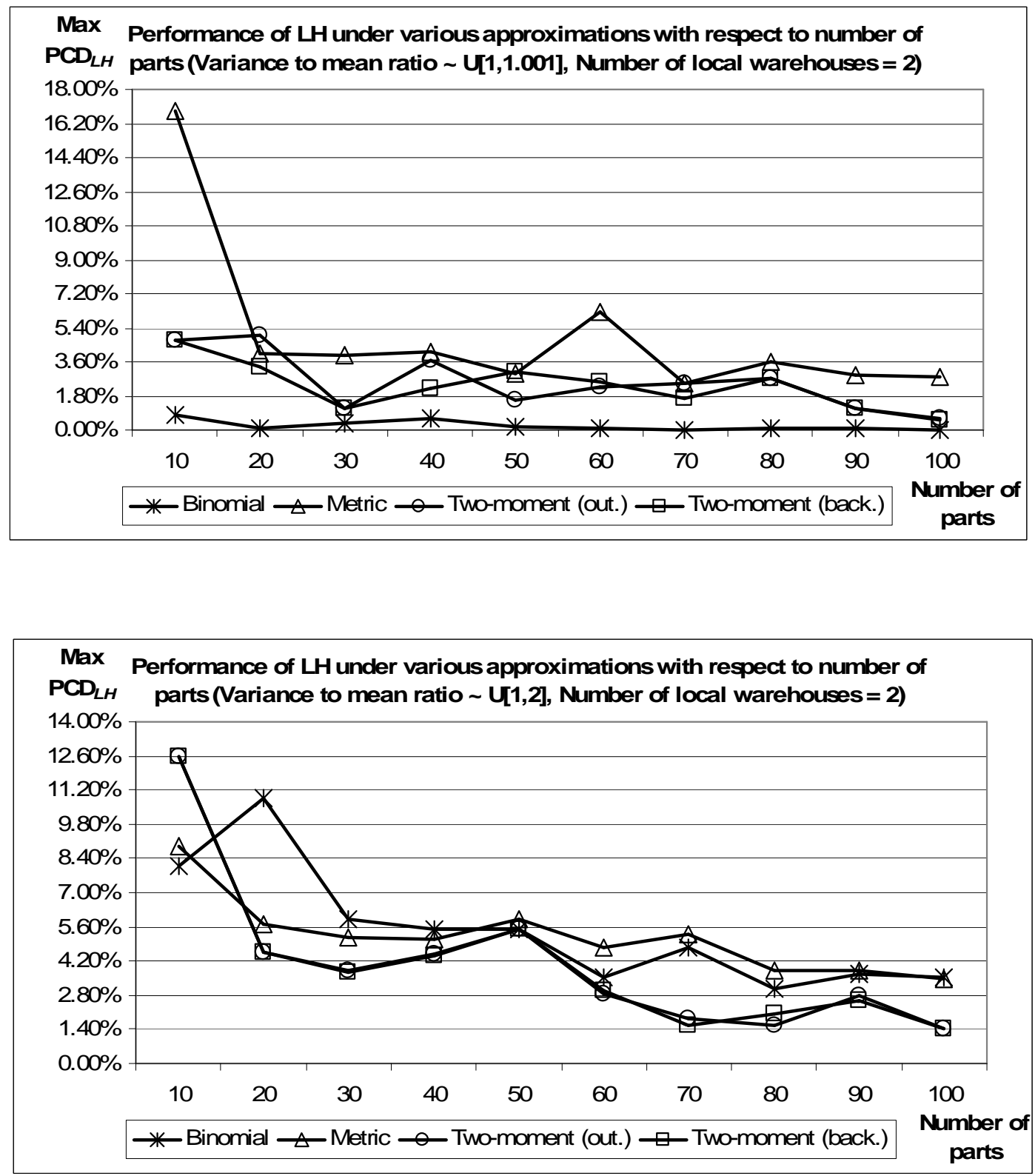

Figure 5.3 (continued) 
son demand case. We interpret this finding as follows: Under the multi-item approach, which makes risk pooling possible among parts, the benefits of risk pooling increases as the number of parts increases. Hence, it is more likely to find a feasible solution close to the optimum by using the Lagrangian heuristic.

- Table 5.7 displays that under all approximations, the Lagrangian heuristic yields more accurate results in terms of the $P C D_{L H}$ when the number of local warehouses is high. Note that for the Poisson demand, we do not have such an observation. Hence, this finding is related with using approximations. That is, binomial disaggregation (binomial approximation), constant lead time assumption (METRIC), and two-moment approximations work better as the number of local warehouses increases. Our findings are in line with Wong et al. (2007b).

- These two observations are important in the sense that the Lagrangian heuristic under the approximate evaluation methods offers a better performance for largesize practical systems. For instance, as shown in Figure 5.3 even in the high demand variance case, both the average and the maximum $P C D_{L H}$ of the solution obtained by the Lagrangian heuristic under the two-moment approximation for outstanding orders decrease down to tolerable levels as the number of parts increases, e.g., when the number of parts is 100 , the average and the maximum $P C D_{L H}$ that the Lagrangian heuristic yields are $0.77 \%$ and $1.41 \%$, respectively for the two-moment approximation for outstanding orders, whereas those for the two-moment approximation for backorders are $0.79 \%$ and $1.44 \%$, respectively.

- As shown in Table 5.7 and Figure 5.3, the binomial disaggregation which is exact in the pure Poisson demand case, yields quite satisfactory results when the variance-to-mean ratio is almost 1 . Nevertheless, when the demand variance is higher, its performance deteriorates and it becomes almost as poor as that of the METRIC. Later, we also show that its performance is even worse than the METRIC when the demand variance is much higher.

- The Lagrangian heuristic yields a better performance for problem instances with

- high average customer arrival rate, 


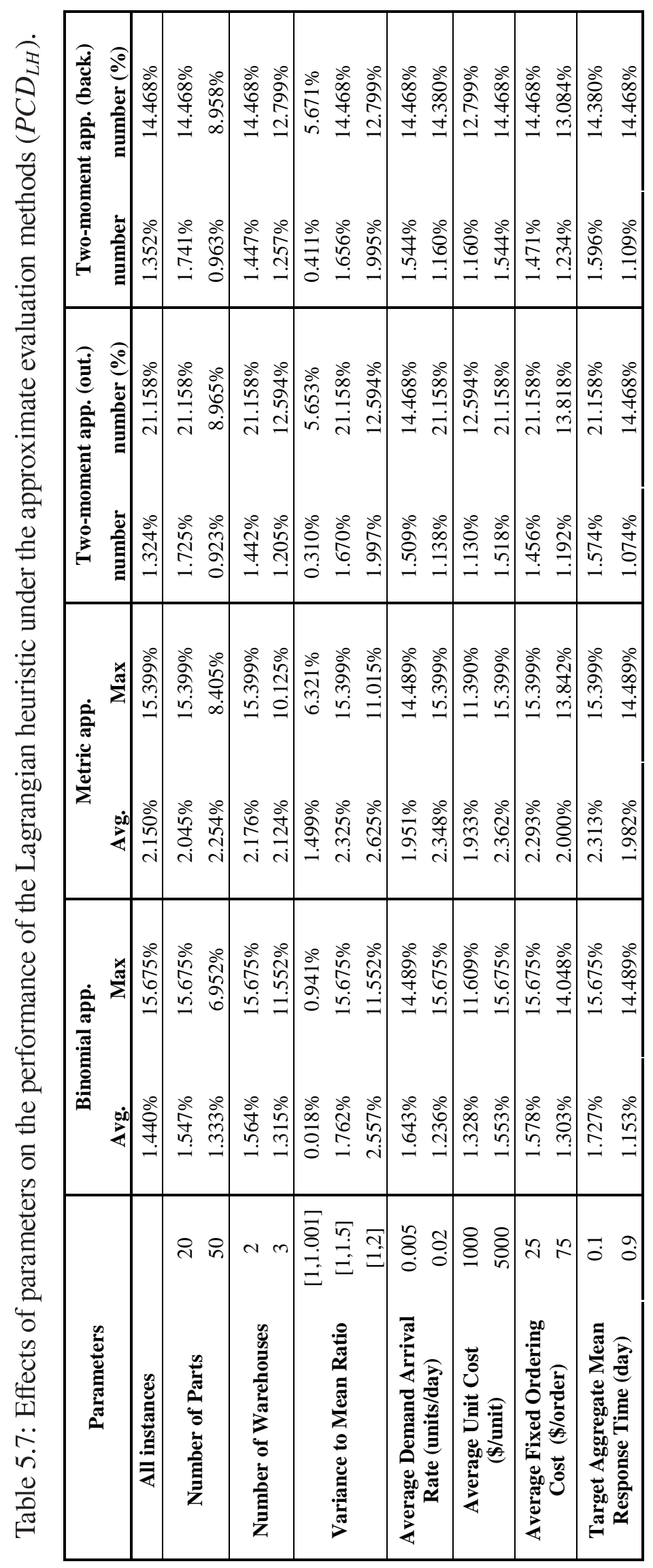


- low average unit variable cost,

- high average fixed ordering cost,

- long target aggregate mean response time.

These findings are common for all approximations, except that the METRIC yields a better performance when the average customer arrival rate is low. These results are in line with the results in pure Poisson demand case in Section 4.4.3. The first three conditions correspond to a situation where the optimal inventory policy parameters, i.e., $Q_{i}, R_{i}$ and $S_{i n}$, are high. This shows that when the value of optimal policy parameters are high, the Lagrangian heuristic performs better. This observation is also in line with Wong et al. (2007b). We interpret the fourth observation as follows: Long target aggregate mean response times yield loose constraints for the problem $P$. Under the multi-item approach, such loose constraints increase the risk pooling among parts. This increases the number of alternative near-optimal solutions. Hence, under long target aggregate mean response times, it is more likely to find a feasible solution that is close to the optimal solution by using the Lagrangian heuristic. The fourth observation is also in line with our findings for the Poisson demand setting in Section 4.4.3.

The main findings based on the feasibility measures that the Lagrangian heuristic yields are as follows:

- As depicted in Tables 5.8, the number of feasible solutions obtained by the Lagrangian heuristic under approximate evaluation methods seems to be relatively few. It decreases with as the following factors increases: the number of parts, the number of local warehouses and the demand variance. Note that these are the most critical factors in our analysis. The former two defines the size of the problems, whereas the latter is a measure specific to the compound Poisson distribution.

- Both the two-moment approximations yield higher performance in terms of the number of feasible solutions than the binomial approximation and METRIC. The two-moment approximation for outstanding orders offers a slightly better performance than the two-moment approximation for backorders. 


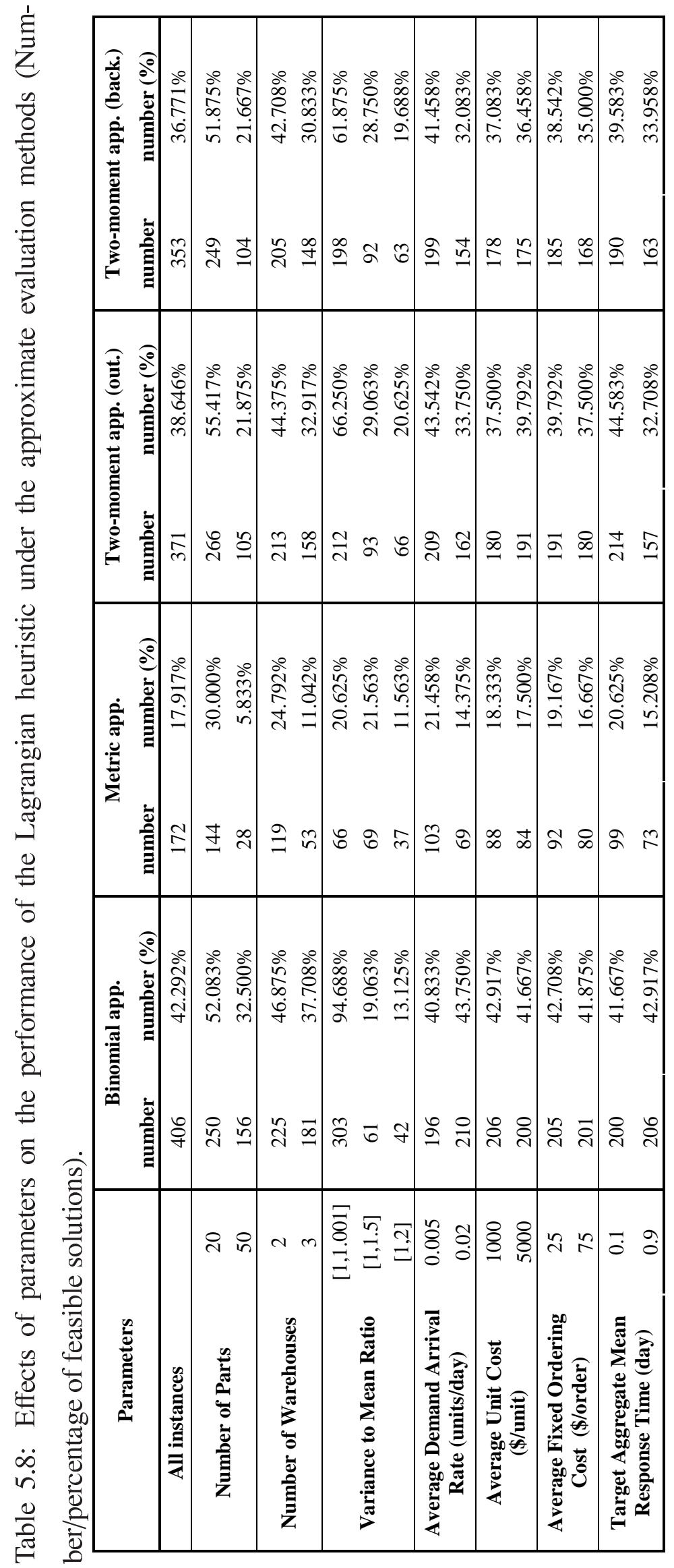


- As shown in Table 5.9, the average RDFR of the solutions obtained by the Lagrangian heuristic under two-moment approximations are quite tolerable and considerably lower than the binomial approximation and the METRIC. However, the maximum RDFR is considerably high. Nevertheless, as shown in 5.4, when either one of the two-moment approximations is used, the average and the maximum RDFR obtained by using the Lagrangian heuristic decrease with the number of parts. For instance, when the number of parts is 100, even in the high demand variance case; when the two-moment approximation for outstanding orders is used, the average and the maximum $R D F R$ of the solutions obtained by the Lagrangian heuristic are $1.81 \%$ and $3.44 \%$, respectively, whereas for the two-moment approximation for backorders the corresponding values are $1.93 \%$ and $3.57 \%$, respectively. This implies when the target aggregate mean response time $W_{n}^{\max }$ is 0.3 days (approximately 7 hours) the two-moment approximation for outstanding orders overshoots this target at most with an average of 15 minutes, which is quite tolerable in practice. The figure reveals that this result improves further as the number of parts increases. The results also show that although the number of infeasible solutions increases with the number of parts, the number of local warehouses and the demand variance, the level of infeasibility, so to say $R D F R$, stays at tolerable levels.

- Similar to our findings for $P C D_{L H}$, the Lagrangian heuristic under the approximate evaluation methods yields a better performance for problem instances with

- high average customer arrival rate,

- low average unit variable cost,

- high average fixed ordering cost,

- long target aggregate mean response time.

Recall that Wong et al. (2007b) considers a decomposition and column generation (DCG) method similar to our Lagrangian heuristic to find the policy parameters of a multi-item two-echelon system operating under a base-stock policy. In this paper, they also consider the METRIC and Graves' two-moment approximation for the analysis of the system. The authors consider the same performance measures as ours 


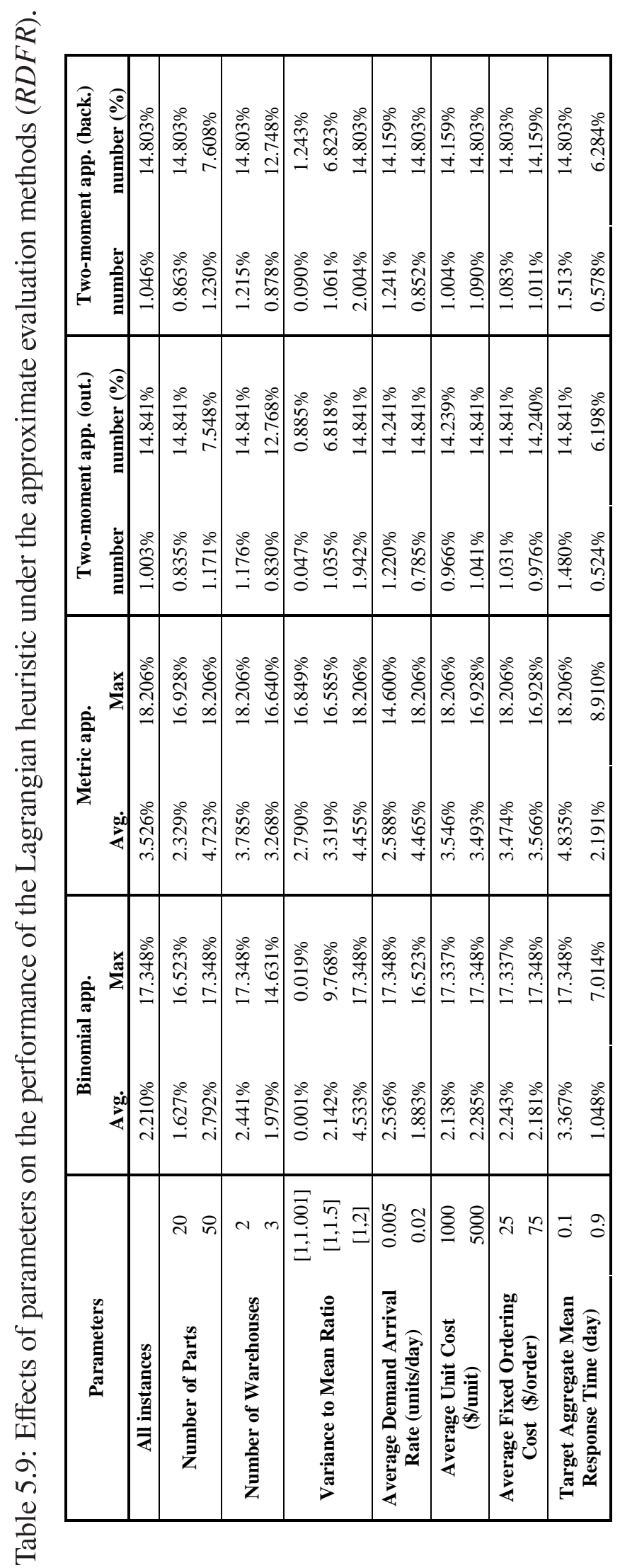



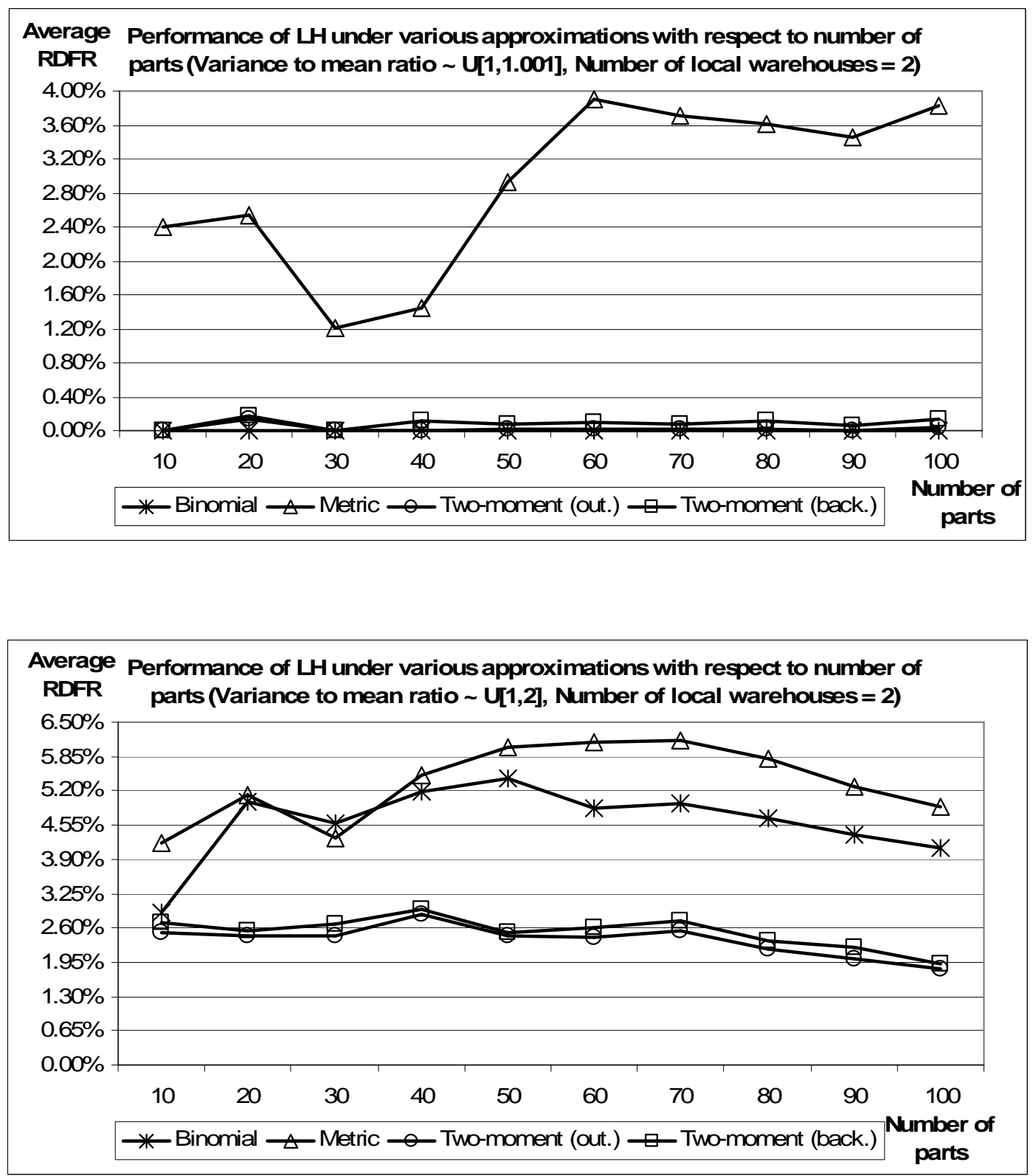

Figure 5.4: Effect of the number of parts on the performance of the Lagrangian heuristic under various approximations (RDFR). 

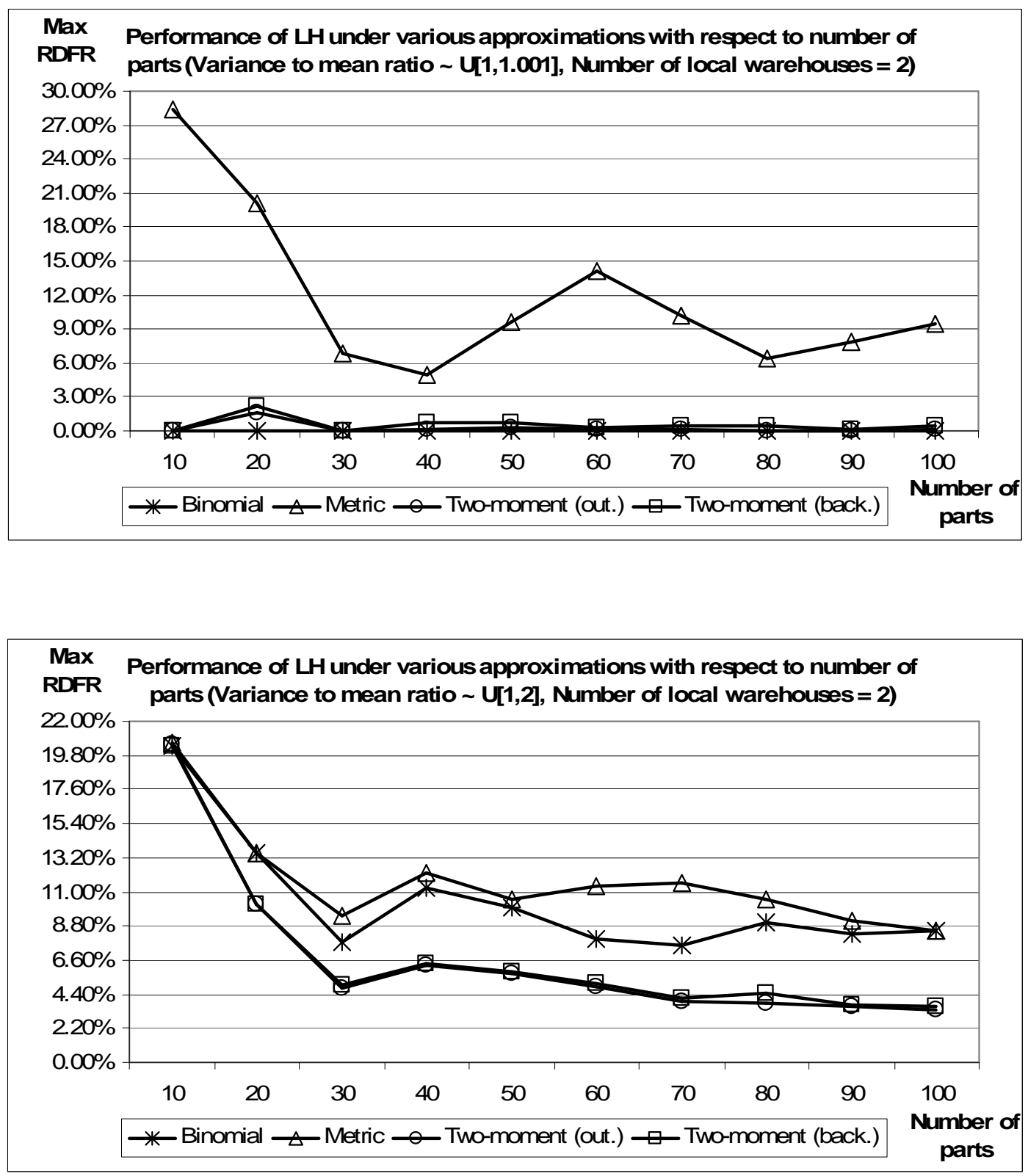

Figure 5.4 (continued) 
to test the approximations. Here, we compare our results with that of Wong et al. (2007b). Accordingly, the relative errors that we obtain in the experiments are considerably higher than that of obtained by the two-moment approximation in Wong et al. (2007b). The relative errors due to using METRIC are only slightly higher than those in Wong et al. (2007b). Due to the computational burden that compound Poisson demand brings, in this work, we have to experiment with relatively small size problems, where the approximations performs worse. This explains the slight difference between the results for METRIC in Wong et al. (2007b) and our work. Nevertheless, the difference between the results for the two-moment approximation in Wong et al. (2007b) and our work is quite significant. That is, the two moment approximation yields an average total difference (in our work, this corresponds to $P C D_{L H}$ ) less than $0.10 \%$ in Wong et al. (2007b), while in our experiments we find that the average $P C D_{L H}$ under the two-moment approximation for outstanding orders is around $1.32 \%$. Similarly, the average and the maximum RDFR under the two-moment approximation in Wong et al. (2007b) are less than $0.11 \%$ and $0.75 \%$, whereas in our experiments the average and the maximum $R D F R$ under the two-moment approximation for outstanding orders are $1.00 \%$ and $14.84 \%$, respectively. We interpret these results as follows: Recall that applying the two-moment approximation to compound Poisson demand setting requires estimation of the variance of the number of outstanding orders $\operatorname{Var}\left(X_{i n}\right)$ and this is quite involved for the compound Poisson distribution, i.e., we obtain $\operatorname{Var}\left(X_{i n}\right)$ by using equation (5.12) and this is only an approximation. Nevertheless, it is easy to obtain the corresponding term in the pure Poisson case (Wong et al. 2007b). The difference between figures in the two works are attributed to the additional approximation required to adapt the two-moment approximation for compound Poisson demand. Since equation (5.12) is used also to implement the twomoment approximation for backorders, this explains the differences in figures for the two-moment approximation for backorders as well.

Finally, we evaluate the resulting stocking levels that the Lagrangian heuristic yields under different approximations. Both the METRIC and the binomial approximation result in understocking. For both approximations, the level of understocking increases even further with the increase in variance-to-mean ratio. Although there are exceptional cases, the two-moment approximations tend to overstock especially 
when the variance-to-mean ratio is high. However, the two-moment approximation for outstanding orders overstocks less compared to the two-moment approximation for backorders. These results also effect the total cost estimations obtained by the approximations in a similar way. For instance, the METRIC and the binomial approximation results underestimate the exact costs, while the two-moment approximations have a tendency to overestimate the real costs. These results are in line with the results reported by Graves (1985), who compares the performance of the two-moment approximation and the METRIC for a base-stock system facing a Poisson demand.

\subsubsection{Performance of the sequential heuristics under the approximate evalu- ation methods}

The results of the experiments for testbed 1 for sequential heuristics $S_{1}, S_{2}$ and $S_{3}$ are summarized in Tables B.1-B.3, B.4-B.6 and B.7-B.9, respectively in Appendix B. The CPU time requirements of the sequential heuristics under the approximate evaluation in the experiment with testbed 1 are depicted in Table 5.10. Based on these results, we make the following observations:

- The average and the maximum $P C D_{L H}$ obtained by the $S_{2}$ and $S_{3}$ heuristics are lower than those of $S_{1}$. This implies that taking the service level requirements into account to obtain the order quantities yields better results for sequential heuristics. The average and the maximum $P C D_{L H}$ obtained by the $S_{3}$ are found to be significantly better than those of $S_{2}$. However, neither of the heuristics dominate the others. All these findings are in line with the findings regarding the performance of the sequential heuristic in the pure Poisson demand case.

- In terms of the $P C D_{L H}$, each of the three sequential heuristics obtained by using the approximate methods yield inferior results compared to the Lagrangian heuristic obtained by using the approximate methods. For instance, under the two-moment approximation for outstanding orders, while the average $P C D_{L H}$ of using the Lagrangian heuristic is only $1.32 \%$, those of $S_{1}, S_{2}$ and $S_{3}$ are $5.64 \%, 4.49 \%, 4.09 \%$, respectively. Nevertheless, this gap decreases as the number of parts or the demand variance increases. However, even for those problem instances that are in favor of the sequential heuristics, the Lagrangian 
heuristic yields quite better results. For instance, when the number of parts is 50, the average $P C D_{L H}$ of using the Lagrangian heuristic is $0.92 \%$, those of $S_{1}, S_{2}$ and $S_{3}$ are $4.13 \%, 2.89 \%$ and $2.62 \%$, respectively. Similarly in the high demand variance case, while the average $P C D_{L H}$ of using the Lagrangian heuristic is $2.00 \%$, those of $S_{1}, S_{2}$ and $S_{3}$ are $5.40 \%, 4.44 \%$ and $3.83 \%$, respectively. Furthermore, the maximum $P C D_{L H}$ of using the sequential heuristics are found to be too high in all of the problem instances.

- In terms of the $P C D_{L H}$, the performances of the approximations do not differ much.

- As opposed to the results in terms of the $P C D_{L H}$, the sequential heuristic yield comparable results with the Lagrangian heuristic in terms of the feasibility measures, e.g., under the two-moment approximation for outstanding orders, while the $R D R F$ of the solution obtained by the Lagrangian heuristic is $1.00 \%$, those of $S_{1}, S_{2}$ and $S_{3}$ are $1.04 \%, 1.03 \%$ and $1.01 \%$, respectively. Intuitively, under any given approximation, no matter what type of heuristic we use, the level of feasibility seems to be the same. Since the approximations mainly affects on the feasibility of the solutions, the results of the sequential and the Lagrangian heuristics turn out to be similar in terms of the measures of feasibility. Nevertheless, since these two heuristics differ in the way they search for the optimal solution, their performances mainly differ in terms of the $P C D_{L H}$.

- In terms of the feasibility measures, the sequential heuristics obtained by applying the two-moment approximations are found to be superior to the one obtained by using the other approximations. The sequential heuristics that are obtained by using the two-moment approximation for outstanding orders yields slightly better results than the one obtained by the two-moment approximation for backorders.

Finally we evaluate the performance of the sequential heuristic in terms of the computational requirements. Table 5.10 summarizes the results. Based on the results, the computational requirements of the sequential heuristics are comparable with the computational requirement of the Lagrangian heuristic given in Table 5.6. This shows that the computational savings of using the sequential heuristics seem to be limited 
compared to that of the Lagrangian heuristic as in the pure Poisson demand case.

\subsubsection{Performance of the Lagrangian dual solution under the approximate eval- uation methods}

The summary of the results for testbed 1 and testbed 2 are presented in Table 5.11 and Figures 5.5, 5.6, respectively. The main findings are as follows:

- As can be seen from both Table 5.11 and Figures 5.5 and 5.6, the Lagrangian dual solution obtained by using the two-moment approximations yields quite satisfactory results. The results are considerably better than those of the Lagrangian dual solutions obtained by using other two approximate evaluation methods. Furthermore, the Lagrangian dual solution implemented by using the two-moment approximation for outstanding orders yields a slightly better performance than the one obtained by using the two-moment approximation for backorders.

- Figures 5.5 and 5.6 reveal that for all the approximations, the PGAP is bounded above. Furthermore, for the two-moment approximations, the average and the maximum $P G A P^{\prime}$ decrease with the number of parts.

- As shown in Table 5.11 and Figures 5.5 and 5.6, the performance of the Lagrangian dual solution obtained by using the approximate methods deteriorates as the demand variance increases. However, Figures 5.5 and 5.6 also show that even in the high demand variance case, the average and the maximum $P G A P^{\prime}$ s are at quite tolerable levels. For instance, when the number of local warehouses is 2 and the number of parts is 100, the average and the maximum $P G A P^{\prime}$ that the two-moment approximation for outstanding orders yields are $0.76 \%$ and $1.17 \%$, while these figures are $0.80 \%$ and $1.19 \%$ for the twomoment approximation for backorders, respectively. Similarly, when the number of local warehouses is 3 and the number of parts is 100 , the average and the maximum $P G A P^{\prime}$ s that the two-moment approximation for outstanding orders

yields are $0.61 \%$ and $1.30 \%$, while these figures are $0.74 \%$ and $1.31 \%$ for the two-moment approximation for backorders, respectively. 


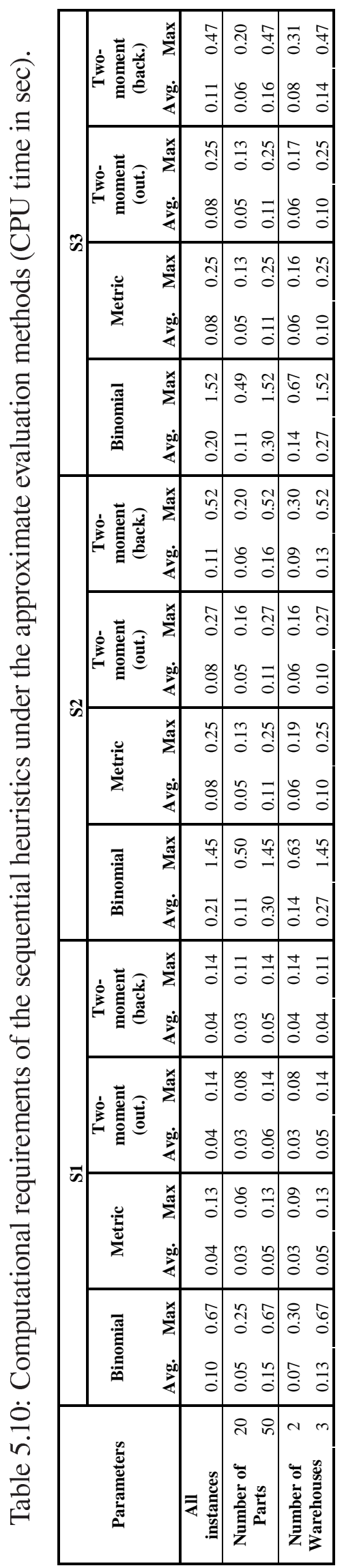




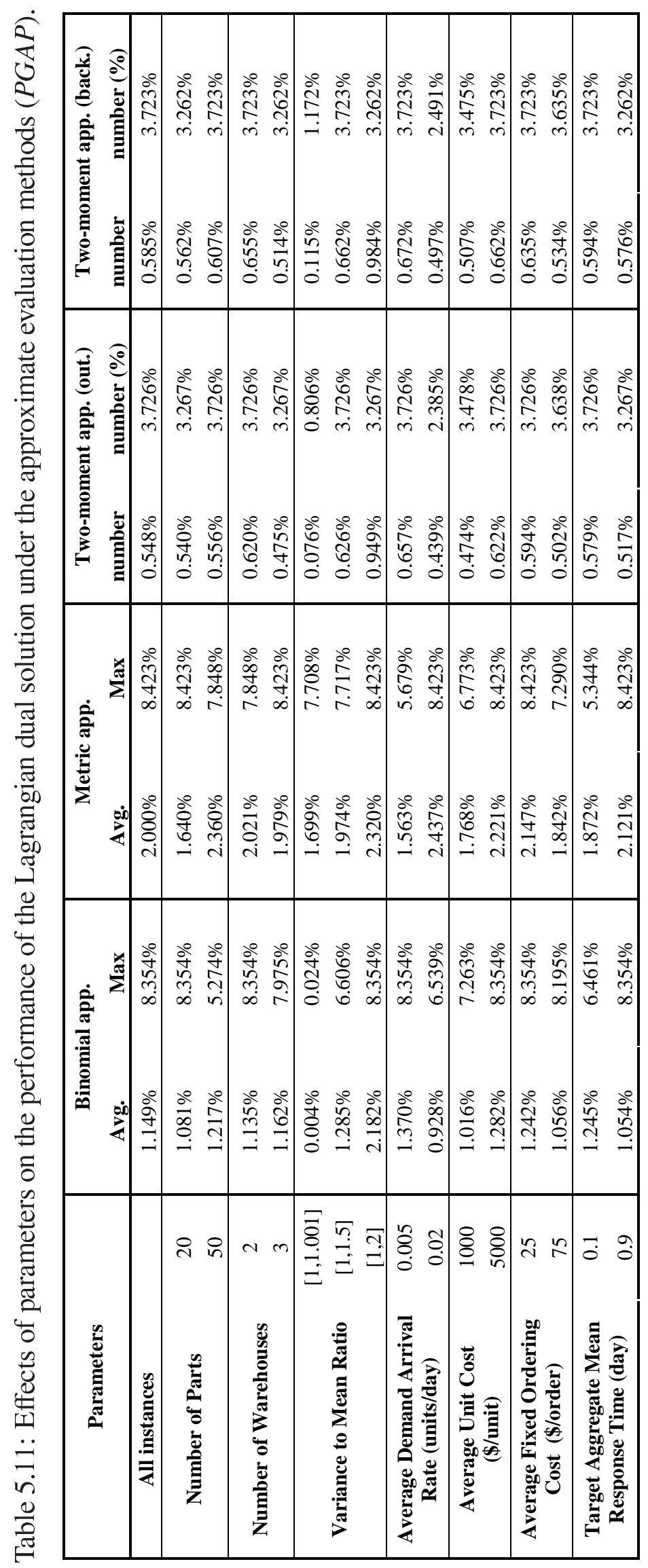


- Similar to our findings for the Lagrangian heuristic obtained by using the approximate evaluation methods in Section 5.5.3.1, the Lagrangian dual solution obtained by using the approximate evaluation methods yields a better performance for problem instances with

- high average customer arrival rate,

- low average unit variable cost,

- high average fixed ordering cost,

- long target aggregate mean response time.

\subsubsection{Performance of the heuristics under the approximate evaluation methods in large-scale problems}

Since the exact Lagrangian dual solution becomes intractable for large practical size problems and the Lagrangian dual solution obtained by using the two-moment approximation for outstanding orders yields satisfactory results in the experiments as discussed in Section 5.5.4, we use the latter as a benchmark solution in our experiments with large-scale problems. We use testbed 3 for this purpose. Figures 5.7-5.10 summarizes the results. Figures 5.7 and 5.9 display the average $P C D_{\text {LHapp }}$ and the average CPU time for the Lagrangian and the sequential heuristics when the two-moment approximation for outstanding orders is used, respectively. Figures 5.8 and 5.10 show the average $P C D_{\text {LHapp }}$ and the average CPU time for the Lagrangian heuristics under the approximations considered in this dissertation. Each point in the figures shows the average of the corresponding measures over 10 problem instances for each setting generated in testbed 3. To illustrate the stocking levels under different approximations, we also analyze the average base-stock levels that the Lagrangian heuristic yields under four different approximations. The results are summarized in Figure 5.7. The main findings are given as follows:

- The average $P C D_{L H a p p}$ of the Lagrangian heuristic obtained by the two-moment approximation for outstanding orders approaches to zero as the number of parts increases. This is intuitive. Under a given approximation, the gap between the Lagrangian heuristic and the Lagrangian dual solution is expected to approach 

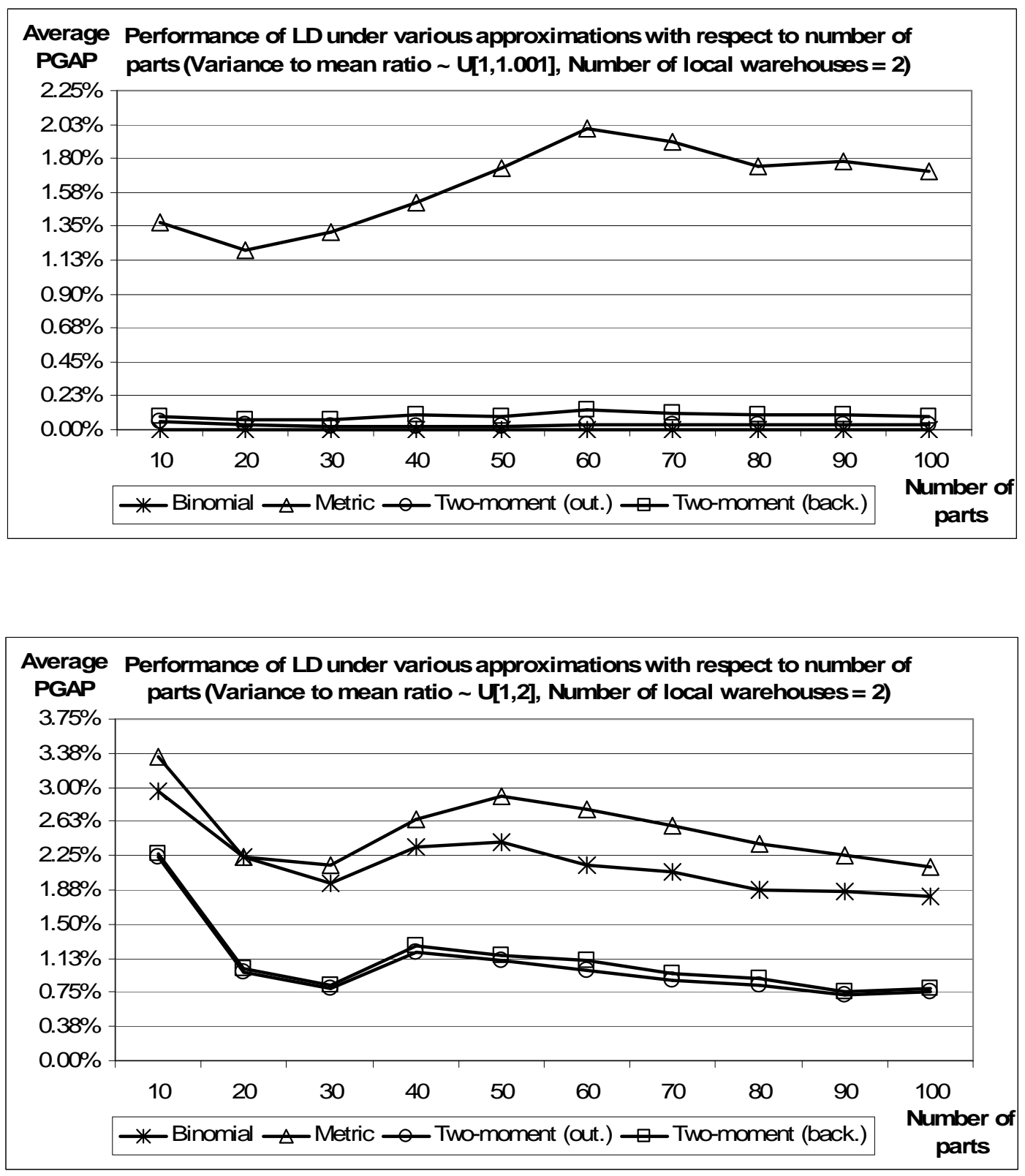

Figure 5.5: Effect of the number of parts on the performance of the Lagrangian dual solution under various approximations $\left(P G A P^{\prime}-\right.$ Number of local warehouses $\left.=2\right)$. 

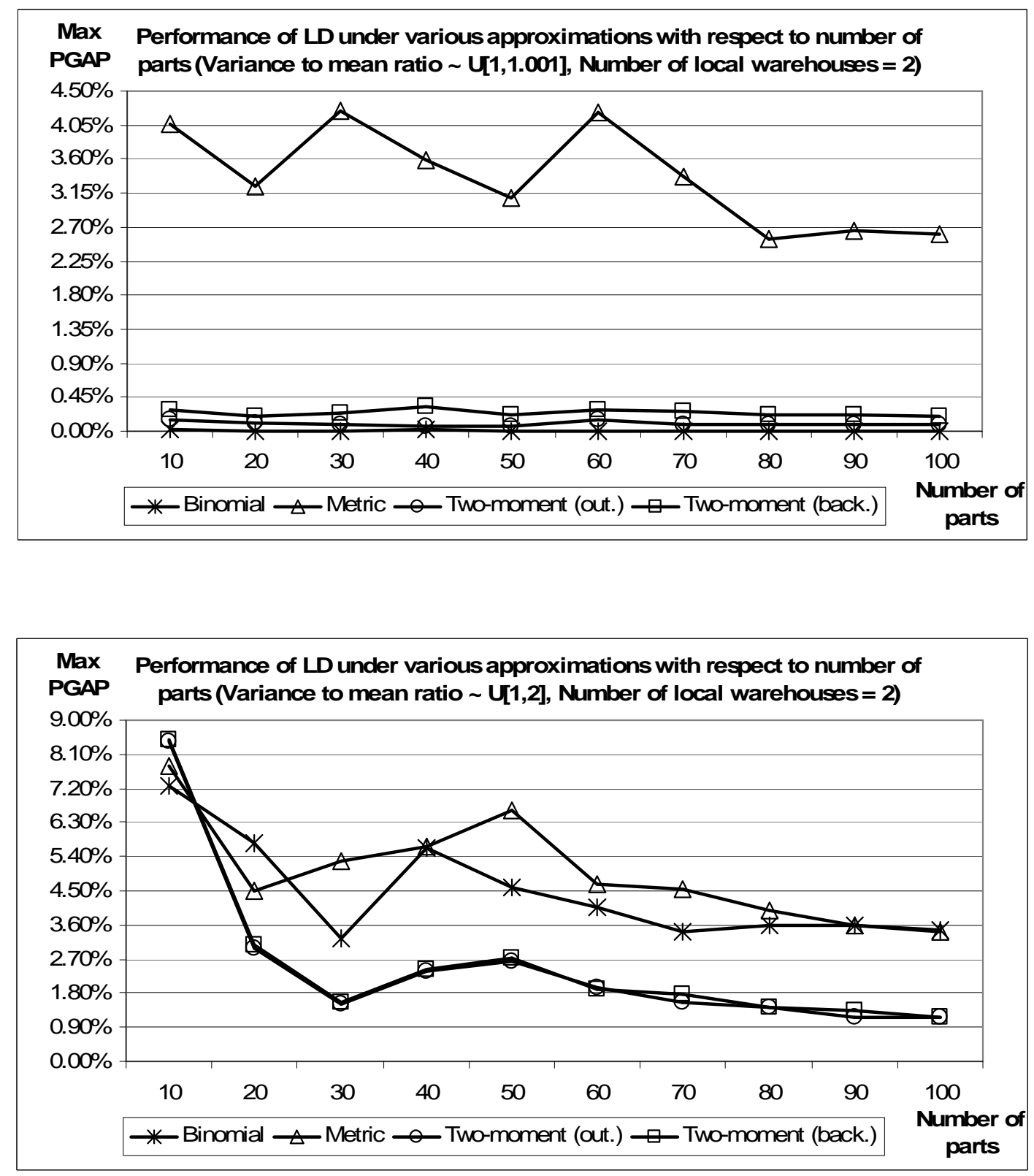

Figure 5.5 (continued) 

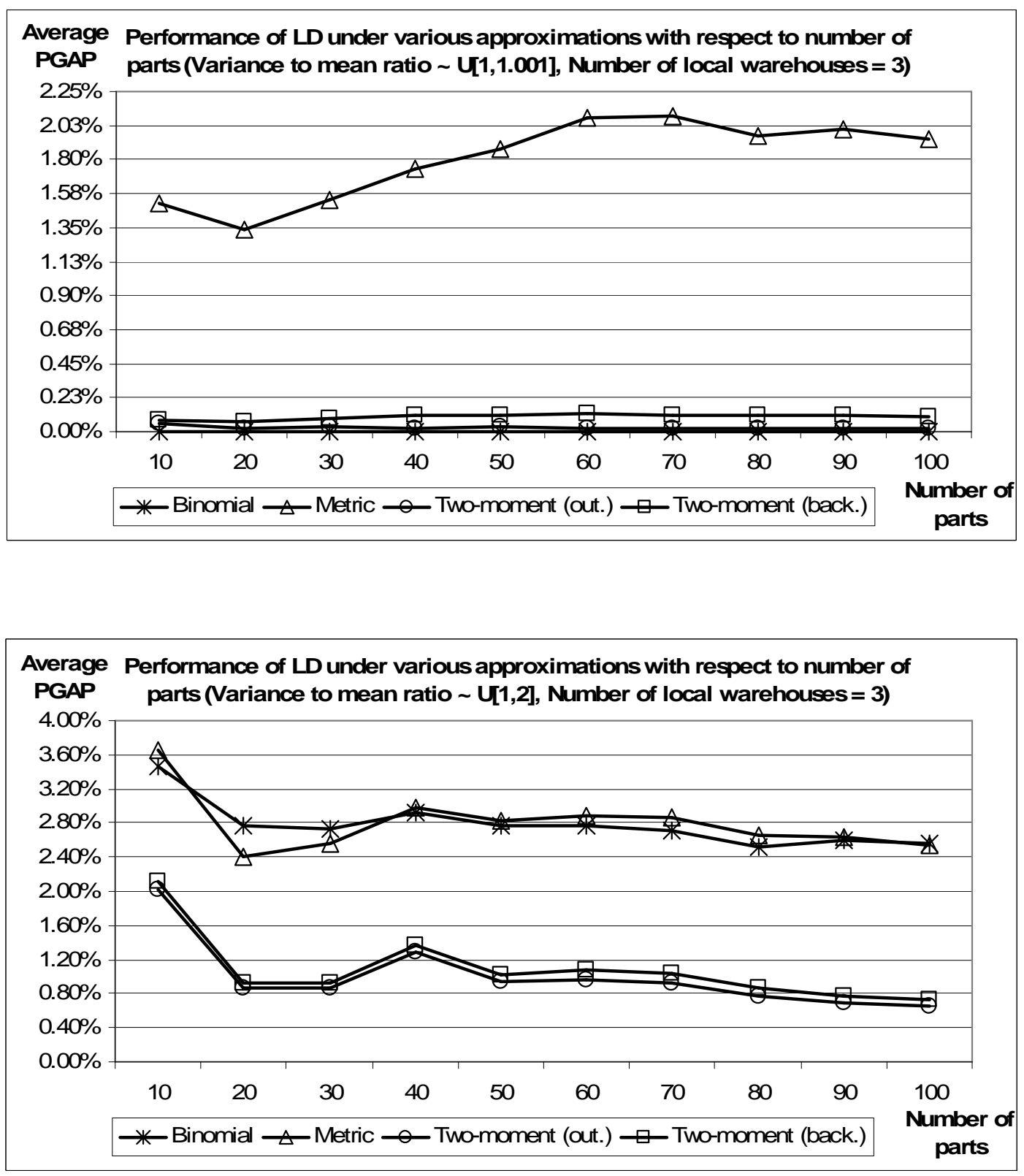

Figure 5.6: Effect of the number of parts on the performance of the Lagrangian dual solution under various approximations $\left(P G A P^{\prime}\right.$ - Number of local warehouses $\left.=3\right)$. 

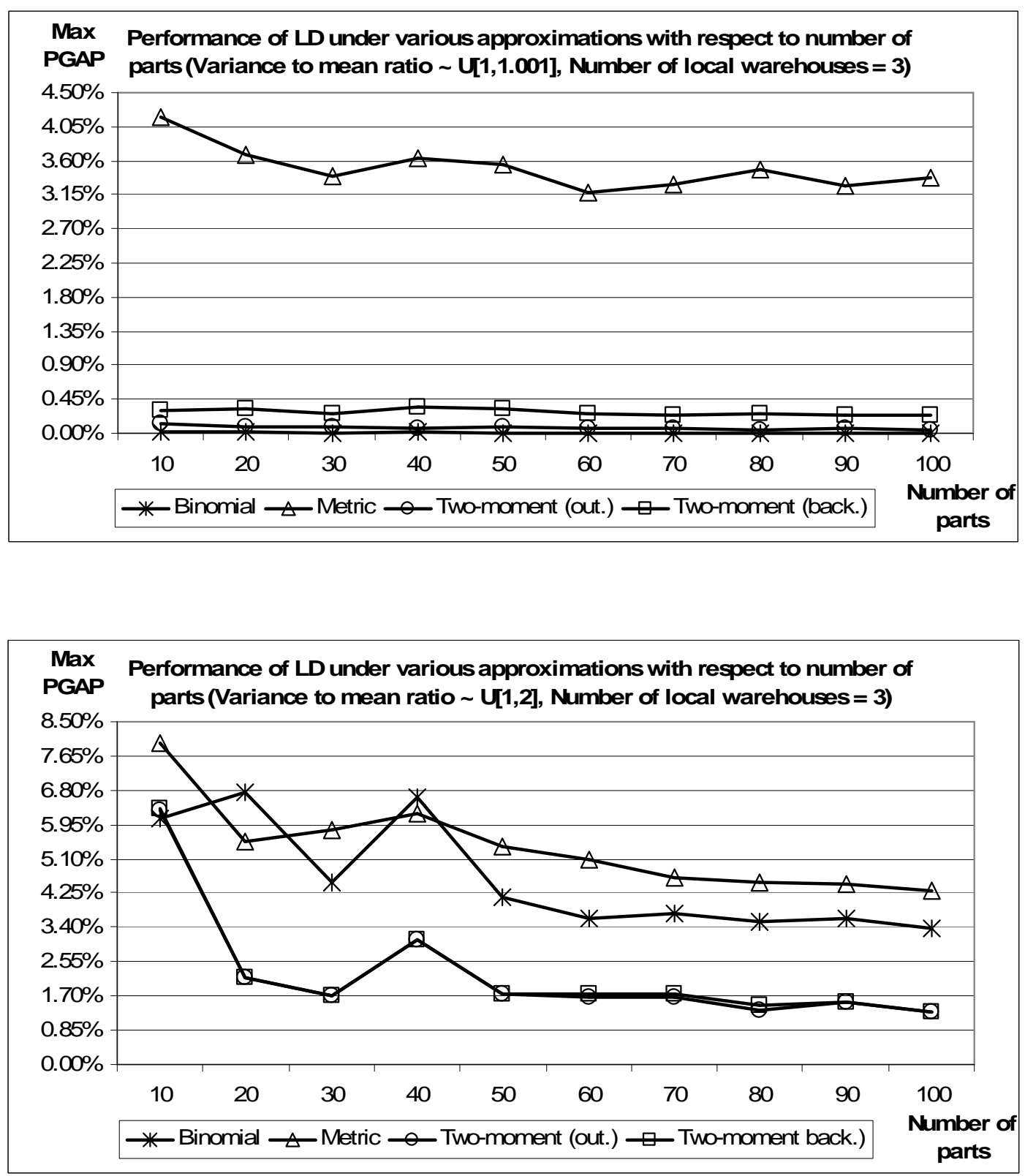

Figure 5.6 (continued) 
to zero as the number of parts increases. We show that this result holds for the exact evaluation case. Now, we observe this result is valid under the twomoment approximation for outstanding orders. The $P C D_{L H a p p}$ of the sequential heuristics decreases with the number of parts as well, but does not converge to zero, e.g., as shown in Figure 5.7, when the number of parts increases to 5,000, the average $P C D_{\text {LHapp }}$ that $S_{1}, S_{2}$ and $S_{3}$ yield approaches to $1.18 \%, 3.22 \%$ and $4.09 \%$, respectively. Also, the average $P C D_{L H a p p}$ that $S_{3}$ yields is found to be quite lower than those of the $S_{1}$ and $S_{2}$. All these findings are is line with the findings in pure Poisson case.

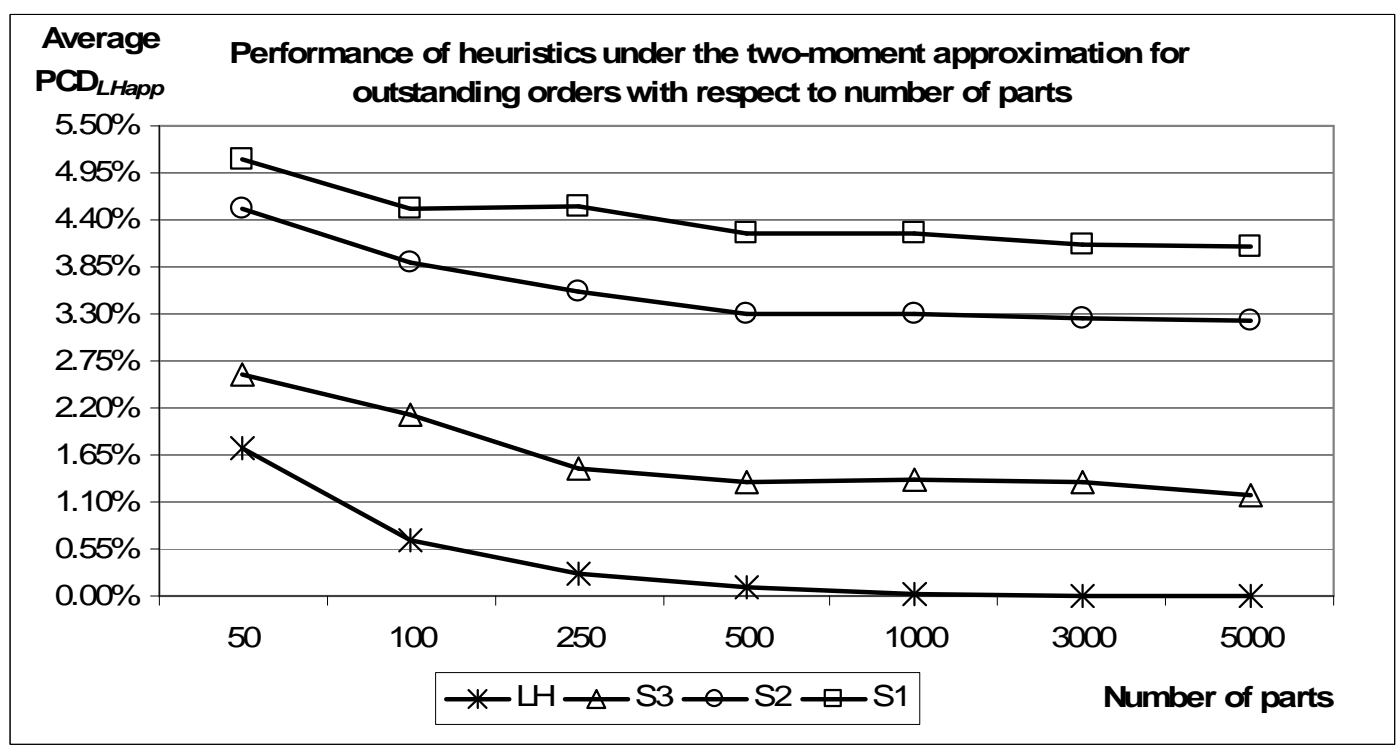

Figure 5.7: Effect of the number of parts on the performance of the Lagrangian and the sequential heuristic under the two-moment approximation for outstanding orders $\left(P C D_{\text {LHapp }}\right)$.

- Figure 5.8 reveals that $P C D_{L H a p p}$ of the Lagrangian heuristic obtained by the two-moment approximation for backorders is also very low. However for the binomial approximation and METRIC, the $P C D_{\text {LHapp }}$ is very high and increases with the number of parts.

- As can be seen from Figure 5.9, the sequential heuristics do not bring a signifi- 

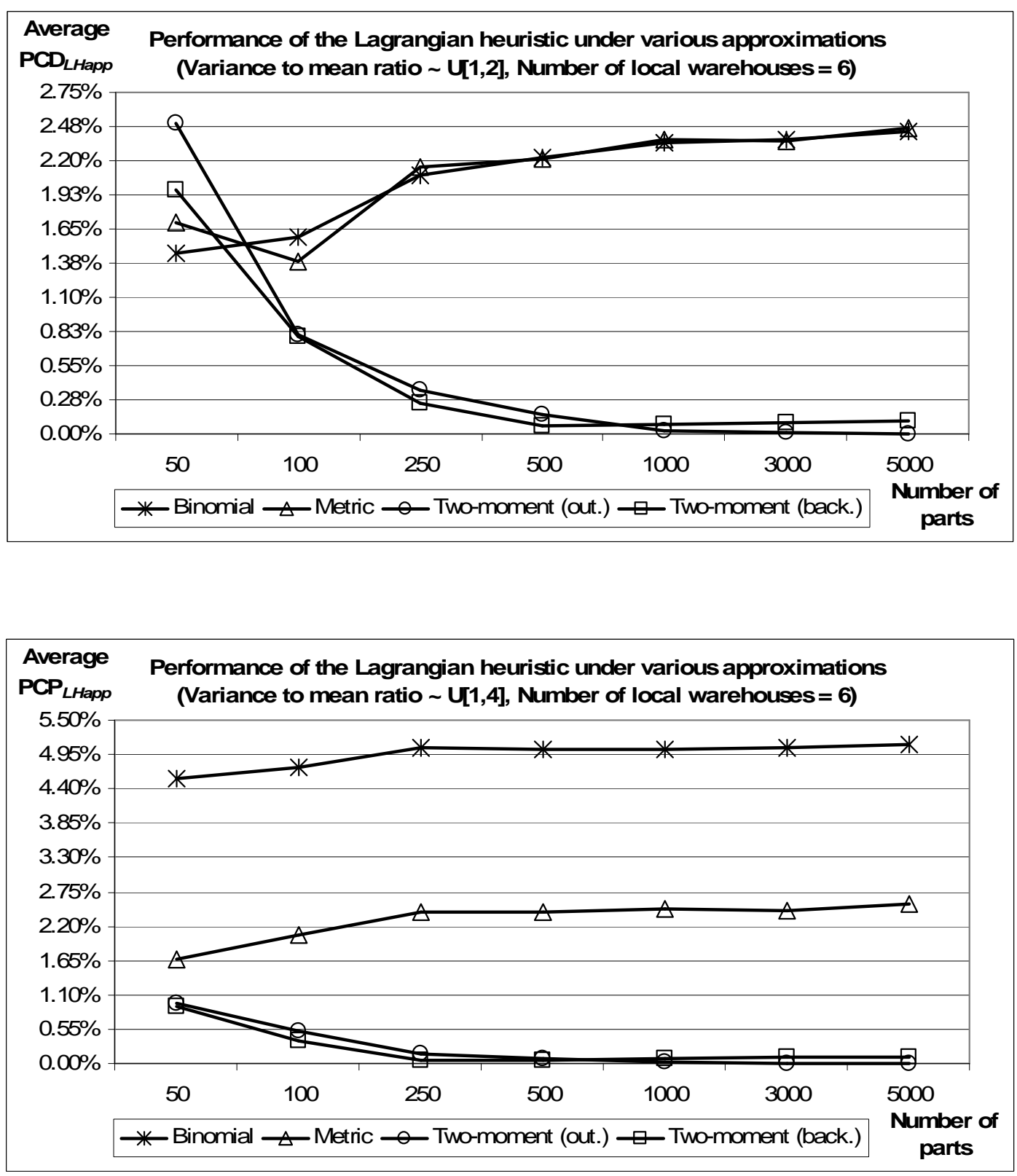

Figure 5.8: Effect of the number of parts on the performance of the Lagrangian heuristic under various approximations $\left(P C D_{\text {LHapp }}\right)$. 
cant computational advantage over the Lagrangian heuristic. Note that this finding is in line with our earlier finding in Section 4.4.5. Figure 5.10 indicates that the two-moment approximation for outstanding orders is quite efficient in terms of the computational requirements; the CPU time required for the Lagrangian heuristic obtained by using the two-moment approximation for outstanding orders is found to be comparable with the one obtained by using METRIC and quite lower than that obtained by using other two approximations.

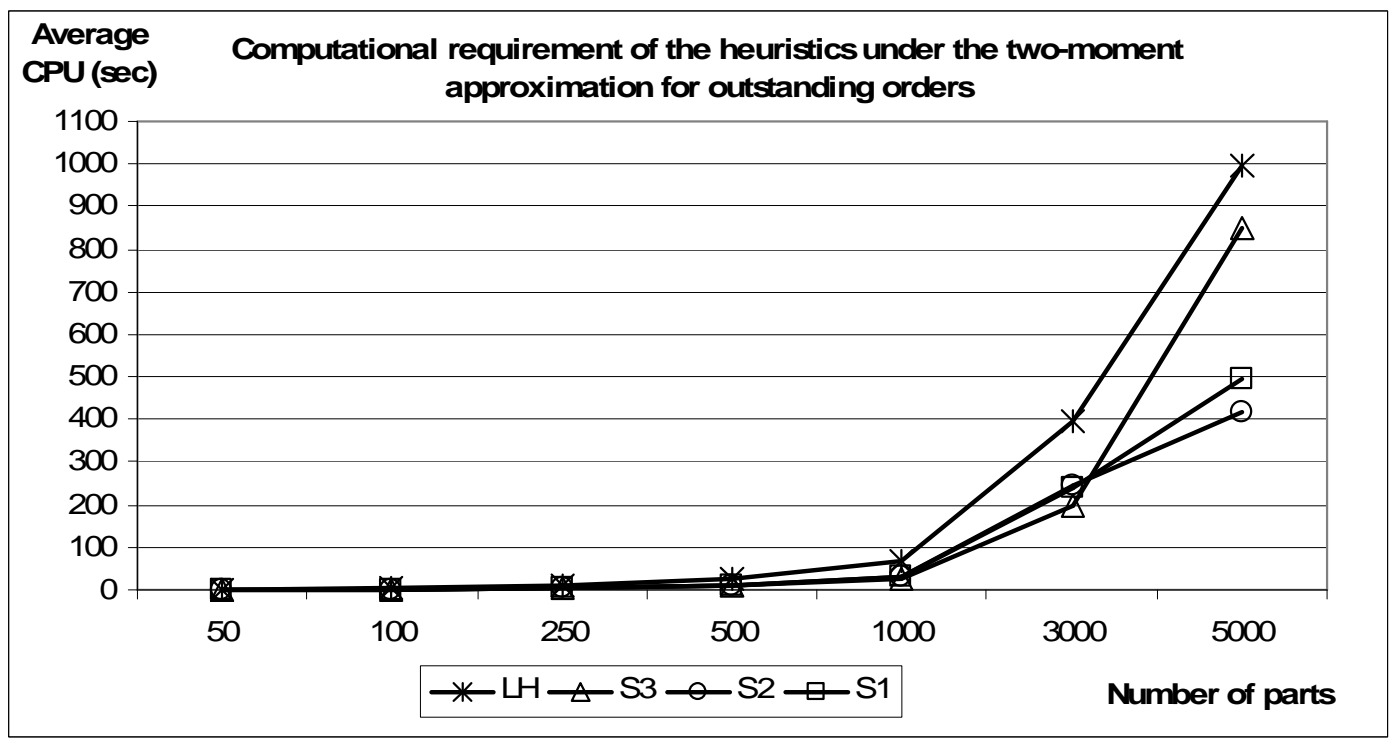

Figure 5.9: Computational requirements of the heuristics evaluated under the twomoment approximation for outstanding orders (CPU time in sec).

- As shown in Figure 5.11, the Lagrangian heuristic yields higher average basestock levels at the local warehouses when either one of the two-moment approximations are used compared to the situation in which the other two approximations are used.

- The results of the experiment for the problem instances with 5,000 parts and 12 local warehouses show that the average $P C D_{\text {LHapp }}$ that the Lagrangian heuristic by using the two-moment approximation for outstanding orders yields is 


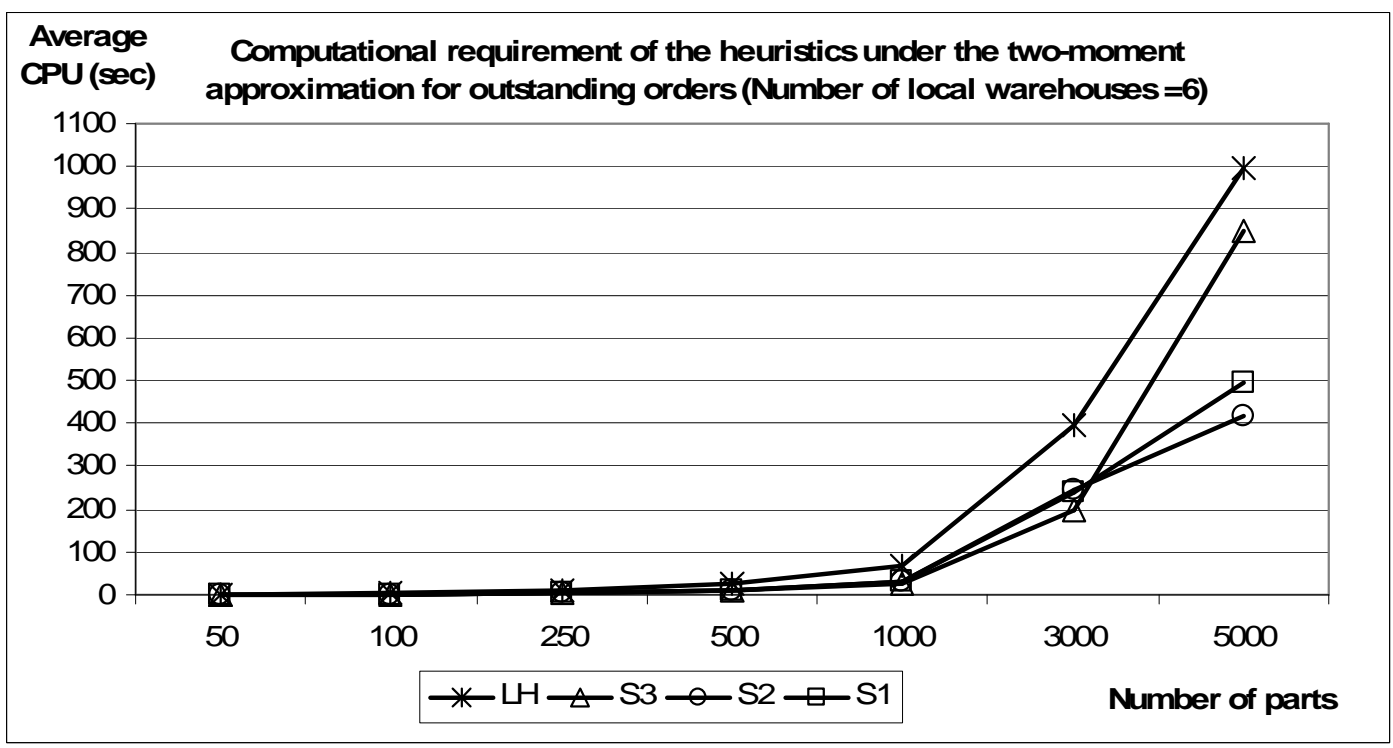

Figure 5.10: Computational requirements of the Lagrangian heuristic under various approximations (CPU time in sec). 
$0.004 \%$, whereas the average CPU time required to obtain the solution of the corresponding heuristic is 1.58 hours. The average $P C D_{L H a p p}$ and the average CPU time for the two-moment approximation for backorders are $0.06 \%$ and 2.97 hours, respectively. These results indicate that under the two-moment approximation, the Lagrangian heuristic is quite efficient in terms of the computational requirements, furthermore, it yields quite promising results.

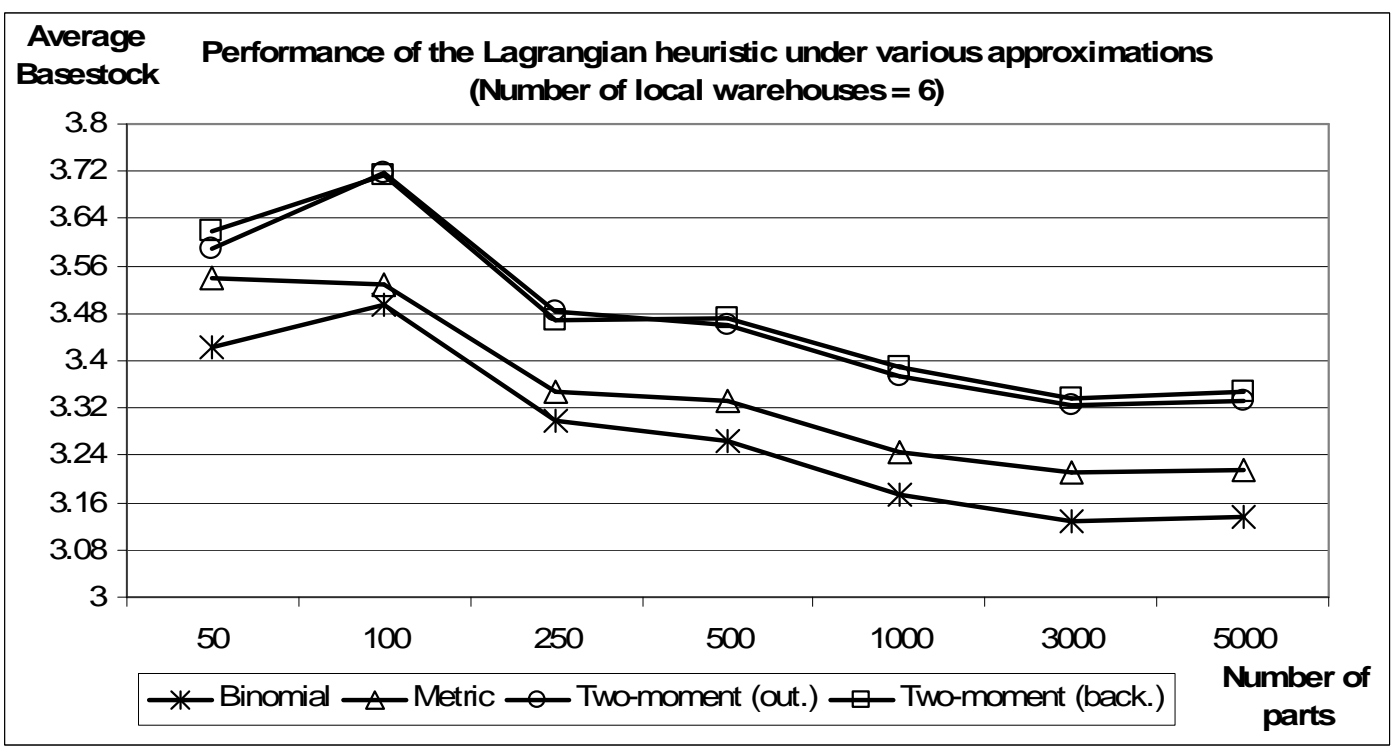

Figure 5.11: Average stocking level obtained by the Lagrangian heuristic evaluated under the two-moment approximation for outstanding orders.

\subsection{Conclusion}

In this work, we extend the Lagrangian heuristic, the sequential heuristics and the Lagrangian dual bound that we develop for the Poisson demand setting in previous chapters to a compound Poisson demand setting. To analyze the steady-state behaviour of the system, we consider an exact and approximate evaluation methods. For the approximate evaluation, we consider four alternatives. The first approxima- 
tion corresponds to the binomial disaggregation method, which is used for the analysis of Poisson demand model in Chapter 3. Note that although is method is exact for the Poisson demand model, it is an approximation for the compound Poisson demand model. For the second approximation and the third approximation, we extend the two-moment approximations proposed by Gallego et al. (2007) and Graves (1985) to our system. In a computational study, we test the performance of the heuristics against the lower bound, the performance of the approximations against the results of exact evaluation method. To the best of our knowledge, our work is the first to propose heuristics for a multi-item two-echelon inventory system facing compound Poisson demand.

The results of the computational study reveal that the Lagrangian heuristic under the exact evaluation method is tractable only for relatively small problems, e.g., problems with 100 parts and 3 local warehouses. The approximations considered in our dissertation are found to be efficient in terms of the computational requirements. The two-moment approximations yield quite satisfactory results compared to the other two approximations. We also investigate the effect of parameters on the performance of the heuristics under the approximate evaluation methods. The performance of the heuristics gets better as the number of parts increases, and it deteriorates as the demand variance increases. Among these two parameters, the effect of number of parts is found to be dominant. This shows that for problem with large number of parts, which is the case in most of the practical application, our heuristics under approximate evaluation methods offer better performances.

We also extend some of the results obtained for the Poisson demand setting to compound Poisson setting: The Lagrangian heuristics under the approximate evaluation methods yields more accurate results compared to the sequential heuristic under the approximate evaluation methods. The Lagrangian heuristic under the two-moment approximation for outstanding orders yield satisfactory results, e.g., when the number of parts is 100 , the average percentage cost difference between the exact Lagrangian heuristic and the Lagrangian heuristic implemented by using the two-moment approximation for outstanding orders is only $0.77 \%$, which is expected to decrease even more as the number of parts increases. The CPU time required by the Lagrangian heuristic is found to be comparable with those of the sequential heuristics. 


\section{CHAPTER 6}

\section{CONCLUSIONS AND FUTURE DIRECTIONS}

In this dissertation, we consider a multi-item two-echelon spare parts inventory distribution problem. This problem can be observed in capital goods manufacturers who provide equipments and services for capital intensive markets. Since the equipments have critical functions, provisioning of the spare parts is of essential importance for the customers. In this environment, it is the manufacturer's responsibility to keep spare parts that will satisfy service requirements. Nevertheless, even in medium-scale inventory system, this requires controlling of thousands of parts, worth of millions of dollars. Therefore, for the manufacturers guaranteeing service requirements and at the same time minimizing the inventory investment is quite essential.

In this dissertation, we consider a multi-item two-echelon spare parts inventory distribution system consisting of a central warehouse operating under a continuous-review installation-stock $(Q, R)$ policy and a number of local warehouses operating under a continuous-review installation-stock $(S-1, S)$ policy. Our objective is to find the optimal or near-optimal policy parameters minimizing the system-wide expected inventory holding and fixed ordering cost subject to an aggregate mean response time constraint at each facility. First, we assume that demand is Poisson. Later, we extend the results that we obtain for the Poisson demand case to compound Poisson setting.

Our work contributes to the literature as follows: First, we propose an exact solution procedure based on a branch-and-price algorithm to find the relevant policy parameters of the system considered. The procedure corresponds to solving the Lagrangian dual problem by using a column generation method, and then using this solution as a lower bound in a branch and bound algorithm. To the best of our knowledge, our 
work is the first to propose an exact solution procedure to find the optimal policy parameters of a multi-item two-echelon inventory system. We experimentally show that the branch-and-price algorithm can be used in applications as long as the number of items and the number of warehouses are limited. Even if this is the case, an exact solution is desirable due to significant cost reductions and benchmark purposes.

We propose four alternative heuristics to find the policy parameters of larger, more practical-size systems. Our heuristics are based on the exact evaluation of the system. The first heuristic, which we call the Lagrangian heuristic, is based on the simultaneous approach and relies on the integration of a column generation method and a greedy algorithm. The other three heuristics are based on the sequential approach, in which first the order quantities are determined using a batch size heuristic, then the reorder levels at the central warehouse and the base-stock levels at the local warehouses are determined through the same method used for the Lagrangian heuristic.

We also propose a lower bound for the optimal system-wide cost based on the column generation and the Lagrangian relaxation. We show that this bound is asymptotically tight in the number of parts. This makes the lower bound very promising for large practical-size problems.

Later, by using our findings and developments as building blocks, we extend our work to a compound Poisson demand setting. To analyze the steady-state behavior of the corresponding system, we consider an exact and approximate evaluation methods. For the approximate evaluation, we consider four alternatives. The first approximation relies on a disaggregation method, which is exact for pure Poisson demand. The other two are the extensions of the two-moment approximations proposed by Graves (1985) and Gallego et al. (2007) to our system setting. The forth one is the extension of the METRIC. To the best of our knowledge, our work is the first to propose heuristics for finding the parameters of a multi-item two-echelon inventory control systems facing a compound Poisson process.

Our major findings can be summarized as follows:

- We empirically show that the performance of the Lagrangian heuristic is quite satisfactory. As the number of parts increases, the performance of the heuris- 
tic improves further, making the heuristic very promising for practical applications. Also the computational requirement of the heuristic is quite tolerable. The experiments with practical size problems show that the heuristic is efficient and effective for large practical industry-size problems.

- The performance of the sequential heuristics are also shown to be satisfactory, but not as much as the Lagrangian heuristic. We also show that some of the qualitative conclusions regarding the performance of the sequential approach in the single-item single-echelon literature do not hold for the multi-item twoechelon setting: The relative cost difference for the sequential heuristics can be quite high compared to the Lagrangian heuristic, which is simultaneous approach based heuristic. The computational advantages of the sequential determination of policy parameters are limited compared to the Lagrangian heuristic.

- Under the compound Poisson demand setting, we show that the exact evaluation of the system is tractable only when the number parts and the number of local warehouses are limited. However, the two-moment approximations that we consider perform well with Lagrangian heuristic, especially when we compare it with the METRIC and the binomial disaggregation. We also extend some of the results that we obtain for the Poisson demand setting to compound Poisson demand setting, e.g., we show that the performance of the heuristics improves with the number of parts, the Lagrangian heuristic is superior to sequential heuristic as in the pure Poisson demand case.

There are many extensions that can be considered for our work. These extensions involve the ones that fit into our work and do not require significant changes in the method that we develop in this dissertation as well as the ones that requires significant changes. The first group of -immediate- extensions are as follows: First, the exact solution procedure, the heuristics and the lower bound developed in the dissertation can be directly extended to systems with target ordering frequency constraints and/or backorder costs. Target ordering frequency constraints make sense in situations where the estimation of fixed ordering costs is difficult. Furthermore, cost models, in which backorder costs are motivated instead of service constraints, are quite common in the literature. Extension of our work to a system with ordering frequency constraints is 
quite immediate since after the relaxation of the target ordering frequency constraints, the corresponding model reduces to ours. This requires introduction of an additional Lagrangian multiplier for each part in the master problem in the column generation method, which is the backbone of the solution method proposed in this dissertation. Similarly, it is quite simple to implement the methods proposed in this dissertation to a cost model since after the relaxation of the service constraints, our problem immediately reduces to a cost model.

It is also possible to extend our work to systems with other service measures such as fill rate, time-based fill rates, i.e., combination of fill rate and response time. These service measures are widely used in practical applications. The extension of our work to these systems requires evaluation of the corresponding service measures. After their evaluation, the solution methods in this dissertation can directly be used to optimize these systems.

Since the Lagrangian heuristic and the Lagrangian dual bound yield quite satisfactory results with practical size problems, they can be used to provide several managerial insights about problems encountered in practice, e.g., cost-benefit analysis of opening up a new local warehouses, determining the optimal number of local warehouses, and cost-benefit analysis of increasing the service levels at the warehouses.

The numerical study with the compound Poisson demand model shows that the performance of the two-moment approximations is not satisfactory as much as in the pure Poisson demand model, hence, there is still room for improvement in the performance of the approximations. Another fruitful research direction is to improve the approximations for the compound Poisson model. For this purpose, one can find a better estimate for the variance of the backorders at the local warehouses. A better approximation integrated with the Lagrangian heuristic can yield quite satisfactory results.

Apart from these immediate extensions, there are several future research directions that requires significant changes in the solution method that we develop in this dissertation. These are as follows: Since both the heuristics and the lower bound that we propose have a general framework, they can be adapted to more complex systems as well, e.g., multi-item, more than two-echelon systems, the ones with more com- 
plex control policies, systems with repairable and condemned parts. In this situation, again, to obtain a lower bound, the column generation can be used to decompose the resulting problem into single-item problems, and the greedy algorithm can be employed to find a feasible solution using the lower bound obtained by the column generation. Similarly, the sequential approach can be extended to more complex systems using the batch size heuristics proposed in this dissertation. For these extensions, the difficulty arises in solving the resulting single-item (multi-echelon) problems, just like the one in this dissertation. Since the structure of the corresponding systems will resemble ours, we expect that one can obtain results similar to ours, such as the asymptotic tightness of the Lagrangian dual bound, and the improvement of the Lagrangian heuristic's performance in the number of parts. Furthermore, it may also be interesting to investigate the issues raised in this dissertation in a more general system setting, e.g., exploring the performance of the sequential approach.

Note that the central warehouse serves two types of customers, the internal and the external customers. This creates a rationing problem for the central warehouse. This rationing problem of the central warehouse can be a quite interesting research issue. Considering that joint ordering, lateral transshipment and emergency shipments are common in practice, investigating the batching decision under joint ordering, lateral transshipments and emergency shipments deserves further investigation. Nevertheless, under these system settings, the analysis of the system and the extension of the methods that we develop in the dissertation will be quite involved. 


\section{REFERENCES}

[1] M.H. Al-Rifai and M.D. Rossetti. An efficient heuristic optimization algorithm for a two-echelon $(R, Q)$ inventory system. Int. J. Production Economics, 109 (1-2), 195-213, 2007.

[2] AMR Research. Service life cycle management (part 1): The approaches and technologies to build sustainable advantages for services. AMR Research, August, 2002.

[3] S. Anily, A. Federgruen. A class of euclidean routing problems with general route cost functions. Mathematics of Operations Research, 15 (2), 268-285, 1990.

[4] S. Axsäter, R. Forsberg, and W.F. Zhang. Approximating general multi-echelon inventory systems by Poisson models. International Journal of Production Economics, 35, 201-206, 1994.

[5] S. Axsäter. Approximate Evaluation of Batch-Ordering Policies for a OneWarehouse, N Non-Identical Retailer System Under Compound Poisson Demand. Naval Research Logistics, 42, 807-819, 1995.

[6] S. Axsäter. Using the Deterministic EOQ Formula in Stochastic Inventory Control. Management Science, 42 (6), 830-834, 1996.

[7] S. Axsäter and W.F. Zhang. Recursive Evaluation of Order-up-to-S Policies for Two-Echelon Inventory Systems with Compound Poisson Demand. Naval Research Logistics, 43, 151-157, 1996.

[8] S. Axsäter. Simple evaluation of echelon stock (R,Q)-policies for two-level inventory systems. IIE Transactions, 29, 661-669, 1997.

[9] S. Axsäter. Evaluation of Installation Stock Based $(R, Q)$ Policies for Two Level Inventory Systems with Poisson Demand. Operations Research, 46 (3), 135145, 1998.

[10] S. Axsäter. Exact Analysis of Continuous Review $(R, Q)$ Policies in Two Echelon Inventory Systems with Compound Poisson Demand. Operations Research, 48 (5), 686-696, 2000.

[11] S. Axsäter. Scaling down multi-echelon inventory problems. International Journal of Production Economics, 71, 255-261, 2001.

[12] S. Axsäter. Approximate Optimization of A Two-Level Distribution Inventory System. International Journal of Production Economics, 81-82, 545-553, 2003.

[13] S. Axsäter. Inventory Control. Springer, New York, 2006. 
[14] C. Barnhart, E.L. Johnson, G.L. Nemhauser, M.W.P. Savelsbergh, and P.H. Vance. Branch and price: column generation for solving huge integer problems. Operations Research, 46 (3), 316-329, 1998.

[15] G.P. Cachon. Exact Evaluation of Batch-Ordering Inventory Policies in TwoEchelon Supply Chains with Periodic Review. Operations Research, 49 (1), 7998, 2001.

[16] D. Çağlar, C.L. Li, and D. Simchi-Levi. Two-echelon spare parts inventory system subject to a service constraint. IIE Transactions, 36, 655-666, 2004.

[17] M. Cohen, P.V. Kamesam, P. Kleindorfer, H. Lee, and A. Tekerian. Optimizer: IBM's multi echelon inventory system for managing service logistics, Interfaces, 20, 65-82, 1990.

[18] M.A. Cohen, Y.S. Zheng, and V. Agrawal. Service parts logistics: a benchmark analysis. IIE Transactions, 29, 627-639, 1997.

[19] K.E. Caggiano, J.A. Muckstadt, and J.A. Rappold. Integrated real-time capacity and inventory allocation for reparable service parts in a two-echelon supply system. Manufacturing and Service Operations Management, 8, 292-318, 2006.

[20] K.E. Caggiano, P.L. Jackson, J.A. Muckstadt, and J.A. Rappold. Optimizing Service Parts Inventory in a Multiechelon, Multi-Item Supply Chain with TimeBased Customer Service-Level Agreements. Operations Research, 55 (2), 303318, 2007.

[21] F. Chen and Y.S. Zheng. One warehouse multi-retailer systems with centralized stock information. Operation Research, 45, 275-287, 1997.

[22] G.B. Dantzig. Linear programming and extensions. Princeton University Press, Princeton, NJ, 1963.

[23] Deloitte. The service revolution in global manufacturing industries. Deloitte Research, 24, 2006.

[24] A. Diaz and M.C. Fu. Multi-echelon models for repairable items: A review. Document in Decision, Operations and Information Technologies Research Works, University of Maryland, 2005.

[25] B. Deuermeyer, L.B. Schwarz. A model for the analysis of system service level in warehouse/retailer distribution systems: the identical retailer case. In L. Schwarz (eds.). Multilevel production/inventory control systems: Theory and Practice, Elsevier, North-Holland, 1981.

[26] A.H.C. Eaves. Forecasting for the ordering and stock- holding of consumable spare parts. Ph.D. Dissertation, University of Lancaster, 2002.

[27] A. Federgruen and Y.Zheng. An efficient algorithm for computing an optimal $(r, q)$ policy in contiuous review stochastic inventory systems. Operations $R e$ search, 40, 808-813, 1992.

[28] G.J. Feeney and C.C. Sherbrooke. The $(S-1, S)$ Inventory Policy Under Compound Poisson Demand. Management Science, 12, 391-411, 1966. 
[29] R. Forsberg. Optimization of order-up-to-S policies for two-level inventory systems with compound Poisson demand. European Journal of Operational Research, 81, 143-153, 1995.

[30] R. Forsberg. Exact Evaluation of $(R, Q)$ Policies for Two-Level Inventory Systems with Compound Poisson Demand. European Journal of Operational Research, 96, 130-138, 1996.

[31] R. Forsberg. Evaluation of $(R, Q)$ policies for two-level inventory systems with generally distributed customer inter-arrival times. European Journal of Operational Research, 99, 401-411, 1997.

[32] G. Gallego. New bounds and heuristics for $(q, r)$ policies. Management Science, 44 (2), 219-233, 1998.

[33] G. Gallego, Ö. Özer, and P. Zipkin. Bounds, Heuristics, and Approximations for Distribution Systems. Operations Research, 55 (3), 503-517, 2007.

[34] M.R. Garey and D.S. Johnson. Computers and Intractability: A Guide to the Theory of NP-Completeness. W.H. Freeman, 1979.

[35] S.C. Graves. A Multi-Echelon Inventory Model for a Repairable Item with Onefor-One Replenishment. Management Science, 31, 1247-1256, 1985.

[36] M. Guignard. Lagrangean Relaxation. Sociedad de Estadistica e Investigacion Operativa Top, 11 (2), 151-228, 2003.

[37] A.T. Gümüş and A.F. Güneri. Multi-echelon inventory management in supply chains with uncertain demand and lead times: Literature review from an operational research perspective. Proc. IMechE Vol. 221 Part B: J. Engineering Manufacture, 1553-1570, 2007.

[38] W.H. Hausman and N.K. Erkip. Multi-Echelon vs. Single-Echelon Inventory Control Policies for Low-Demand Items. Management Science, 40 (5), 597-602, 1994.

[39] W.J. Hopp, M.L. Spearman, and R.Q. Zhang. Easily Implementable Inventory Control Policies. Operations Researchs, 45, 327-340, 1997.

[40] W.J. Hopp, R.Q. Zhang, and M.L. Spearman. An easily implementable hierarchical heuristic for a two-echelon spare parts distribution system. IIE Transactions, 31, 977-988, 1999.

[41] K. Kaas, M. Goovaerts, J. Dhaene, and M. Denuit. Modern Actuarial Risk Theory. Kluwer Academic Publishers, 2001.

[42] H. Kellerer, U. Pferschy, and D. Pisinger. Knapsack Problems. Springer, Berlin, 2004

[43] W.J. Kennedy, J. Wayne Patterson, and D.F. Lawrence. An overview of recent literature on spare parts inventories. International Journal of Production Economics, 76 (2), 201-215, 2002.

[44] S.H. Kim, M.A. Cohen, and S. Netessine. Reliability or Inventory? Analysis of Product Support Contracts in the Defense Industry. Working Paper, 2009 
[45] H.L. Lee and K. Moinzadeh. A Multi-Echelon Repairable Inventory Model with Batch Ordering Policy. Technical Report, No. 84-4, Department of Industrial Engineering and Engineering Management, Stanford University, Stanford, California, 1984a

[46] H.L. Lee and K. Moinzadeh. Operating Charactersitics of a Two-Echelon Inventory System for Repairable and Consumable Items under Batch Ordering and Shipment Policy. Technical Report, No. 84-6, Department of Industrial Engineering and Engineering Management, Stanford University, Stanford, California, $1984 \mathrm{~b}$

[47] M.E. Lübbecke and J. Desrosiers. Selected Topics in Column Generation. Operations Research, 53(6), 1007-1023, 2002.

[48] K. Moinzadeh and H.L. Lee. Batch size and stocking levels in multi-echelon repairable systems. Management Science, 32 (12), 1567-1581, 1986.

[49] Ö. Özer and H. Xiong. Stock positioning and performance estimation for distribution systems with service constraints. IIE Transactions, 40 (12), 1141-1157, 2008.

[50] A.H.G. Rinnooy Kan, L. Stougie, and C. Vercellis. A class of generalized greedy algorithms for the multi-knapsack problem. Discrete Applied Mathematics, 42 279-290, 1993.

[51] H.E. Romeijn and D.R. Morales. A class of greedy algorithms for the generalized assignment problem. Discrete Applied Mathematics, 103, 209-235, 2000.

[52] S.M. Ross. Stochastic Processes. Springer, John Wiley and Sons, New York, NY, 1996.

[53] K. Shanker. Exact analysis of a two-echelon inventory system for recoverable items under batch inspection policy. Naval Research Logistics Quarterly, 28, 579-601, 1981.

[54] C.C. Sherbrooke. A multi-echelon technique for recoverable item control. $O p$ erations Research, 16, 122-141, 1968.

[55] E.A. Silver, D. Pyke, and R. Peterson. Inventory Management and Production Planning and Scheduling, Wiley, New York, 1998.

[56] D. Simchi-Levi and Y. Zhao. Three Generic Methods for Evaluating Stochastic Multi-Echelon Inventory Systems. Working paper, 2010.

[57] D. Simchi-Levi and Y. Zhao. Safety stock positioning in supply chains with stochastic lead times. Manufacturing Service Opererations Management, 7, 295-318, 2005.

[58] A. Sleptchenko, M. C. Van der Heijden, and A. Van Harten. Effects of finite repair capacity in multi-echelon, multi-indenture service part supply systems. International Journal of Production Economics, 79 (3), 209-230, 2002.

[59] U.W. Thonemann, A.O. Brown, and W.H. Hausman. Easy Quantification of Improved Spare Parts Inventory Policies. Management Science, 48 (9), 1213-1225, 2002. 
[60] E. Topan, Z.P. Bayındır, and T. Tan. An exact solution procedure for multi-item two-echelon spare parts inventory control problem with batch ordering in the central warehouse. Operations Research Letters, 38, 454-461, 2010 a.

[61] E. Topan, Z.P. Bayındır, and T. Tan. Heuristics for Multi-Item Two-Echelon Spare Parts Inventory Control Problem with Batch Ordering in the Central Warehouse, Working paper, 2010b.

[62] E. Topan, Z.P. Bayındır, and T. Tan. Heuristics for Multi-Item Two-Echelon Spare Parts Inventory Control Problem with Batch Ordering in the Central Warehouse Under Compound Poisson Demand, Working paper, 2010c.

[63] D.M. Topkis. Supermodularity and Complementarity. Princeton University Press, Princeton, NJ, 1998.

[64] G.J. Van Houtum, A. Scheller-Wolf, J. Yi. Optimal control of serial inventory systems with fixed replenishment intervals. Operations Research, 55 (4), 674687, 2007.

[65] H. Wong, G.J. Van Houtum, D. Cattrysse, and D. Van Oudheusden. Simple, efficient heuristics for multi-item multi-location spare parts systems with lateral transshipments and waiting time constraints. Journal of the Operational Research Society, 56, 1419-1430, 2005.

[66] H. Wong, G.J. Van Houtum, D. Cattrysse, and D. Van Oudheusden. Multi-item spare parts systems with lateral transshipment and waiting time constraints. $E u$ ropean Journal of Operational Research, 71, 1071-1093, 2006.

[67] H. Wong, D. Van Oudheusden, and D. Cattrysse. Two-Echelon Multi-Item Spare Parts Systems with Emergency Supply Flexibility and waiting Time Constraints. IIE Transactions, 39, 1045-1057, 2007a.

[68] H. Wong, D. Kranenburg, G.J. Van Houtum, and D. Cattrysse. Efficient heuristics for two-echelon spare parts inventory systems with an aggregate mean waiting time constraint per local warehouse. OR Spectrum, 29, 699-722, $2007 \mathrm{~b}$.

[69] Y.S. Zhao. Evaluation and Optimization of Installation Base-Stock Policies in Supply Chains with Compound Poisson Demand. Operations Research, 56 (2), 437-452, 2008.

[70] Y.S. Zheng. On Properties of Stochastic Inventory Systems. Management Science, 38 (1), 87-103, 1992.

[71] P.H Zipkin. Foundations of Inventory Management. McGraw- Hill, NewYork, 2000. 


\section{APPENDIX A}

\section{EXTENDING THE SOLUTION PROCEDURE FOR SUBPROBLEMS TO COMPOUND POISSON DEMAND SETTING}

\section{A.1 Solution Procedure for Subproblems: Single-item Two-echelon Batch Or- dering Problem}

In this part, we develop the theoretical framework for the solution procedure in Section 5.4. In this way, we extend the results in Section 3.2.2 to compound Poisson setting. As in Section 3.2.2, our analysis is based on the notions of stochastic domination and supermodularity (see Ross, 1996 and Topkins, 1998).

Lemma A.1.1 For any $Q_{i}^{+}>Q_{i}$ and $R_{i}^{+}>R_{i}$,

a) $X_{\text {in }}\left(Q_{i}, R_{i}\right) \geq_{s t} X_{\text {in }}\left(Q_{i}, R_{i}^{+}\right)$,

b) $X_{\text {in }}\left(Q_{i}, R_{i}\right) \geq_{s t} X_{\text {in }}\left(Q_{i}^{+}, R_{i}\right)$,

where $\geq_{s t}$ denotes stochastic dominance, and $\leq_{s t}$ is defined similarly.

Proof. Using equation (5.8), it is easy to show that $P\left\{B_{i 0}\left(Q_{i}, R_{i}\right) \leq x\right\} \leq P\left\{B_{i 0}\left(Q_{i}, R_{i}^{+}\right)\right.$ $\leq x\}$ or equivalently $B_{i 0}\left(Q_{i}, R_{i}\right) \geq_{s t} B_{i 0}\left(Q_{i}, R_{i}^{+}\right)$. Similarly, we have $B_{i 0}\left(Q_{i}, R_{i}\right) \geq_{s t}$ $B_{i 0}\left(Q_{i}^{+}, R_{i}\right)$. Since the internal demand from different warehouses are not differentiated at the central warehouse, these results hold for $B_{i 0}^{n}\left(Q_{i}, R_{i}\right)$, i.e., $B_{i 0}^{n}\left(Q_{i}, R_{i}\right) \geq_{s t}$ $B_{i 0}^{n}\left(Q_{i}, R_{i}^{+}\right)$and $B_{i 0}^{n}\left(Q_{i}, R_{i}\right) \geq_{s t} B_{i 0}^{n}\left(Q_{i}^{+}, R_{i}\right)$. Since $X_{i n}\left(Q_{i}, R_{i}\right)=B_{i 0}^{(n)}\left(Q_{i}, R_{i}\right)+Y_{i n}$, the results also hold for $X_{i n}\left(Q_{i}, R_{i}\right)$. 
Lemma A.1.2 For any $Q_{i}^{+}>Q_{i}, R_{i}^{+}>R_{i}, S_{i n}^{+}>S_{i n}$, the difference function $\underset{S_{i n}}{\nabla}\left(Q_{i}, R_{i}\right)$ $=I_{\text {in }}\left(Q_{i}, R_{i}, S_{\text {in }}^{+}\right)-I_{\text {in }}\left(Q_{i}, R_{i}, S_{\text {in }}\right)$ satisfies

a) $\underset{S_{\text {in }}}{\nabla}\left(Q_{i}, R_{i}\right) \unlhd_{s t} \underset{S_{\text {in }}}{\nabla}\left(Q_{i}, R_{i}^{+}\right)$,

b) $\underset{S_{\text {in }}}{\nabla}\left(Q_{i}, R_{i}\right) \unlhd_{s t} \underset{S_{\text {in }}}{\nabla}\left(Q_{i}^{+}, R_{i}\right)$.

Proof. Proof of part (a) and (b) follows from Lemma A.1.1(a) and (b), respectively. The rest of the proofs are the same as that of Lemma 3.2.2 in Section 3.2.2.

Corollary A.1.3 For any $Q_{i}^{+}>Q_{i}, R_{i}^{+}>R_{i}$ and $S_{i n}^{+}>S_{i n}$,

a) $E\left[I_{\text {in }}\left(Q_{i}, R_{i}, S_{\text {in }}^{+}\right)\right]-E\left[I_{\text {in }}\left(Q_{i}, R_{i}, S_{\text {in }}\right)\right] \leq E\left[I_{\text {in }}\left(Q_{i}, R_{i}^{+}, S_{\text {in }}^{+}\right)\right]-E\left[I_{\text {in }}\left(Q_{i}, R_{i}^{+}, S_{\text {in }}\right)\right]$, i.e., $E\left[\underset{S_{\text {in }}}{\nabla}\left(Q_{i}, R_{i}\right)\right] \leq E\left[\underset{S_{\text {in }}}{\nabla}\left(Q_{i}, R_{i}^{+}\right)\right]$,

b) $E\left[I_{\text {in }}\left(Q_{i}, R_{i}, S_{\text {in }}^{+}\right)\right]-E\left[I_{\text {in }}\left(Q_{i}, R_{i}, S_{\text {in }}\right)\right] \leq E\left[I_{\text {in }}\left(Q_{i}^{+}, R_{i}, S_{\text {in }}^{+}\right)\right]-E\left[I_{\text {in }}\left(Q_{i}^{+}, R_{i}, S_{\text {in }}\right)\right]$, i.e., $E\left[\underset{S_{\text {in }}}{\nabla}\left(Q_{i}, R_{i}\right)\right] \leq E\left[\underset{S_{\text {in }}}{\nabla}\left(Q_{i}^{+}, R_{i}\right)\right]$.

Theorem A.1.4 $G\left(Q_{i}, R_{i}, \vec{S}_{i}\right)$ is supermodular in

a) $R_{i}$ and $\overrightarrow{S_{i}}$,

b) $Q_{i}$ and $\overrightarrow{S_{i}}$.

Proof. Proof of part (a): To show the supermodularity of $G\left(Q_{i}, R_{i}, \overrightarrow{S_{i}}\right)$ in $R_{i}$ and $\overrightarrow{S_{i}}$, it suffices show that for a given value of $Q_{i}, G\left(Q_{i}, R_{i}, \overrightarrow{S_{i}^{+}}\right)-G\left(Q_{i}, R_{i}, \overrightarrow{S_{i}}\right) \leq$ $G\left(Q_{i}, R_{i}^{+}, \overrightarrow{S_{i}^{+}}\right)-G\left(Q_{i}, R_{i}^{+}, \overrightarrow{S_{i}}\right)$ for any ${\overrightarrow{S_{i}}}^{+}>\vec{S}_{i}$ and $R_{i}^{+}>R_{i}$. First, by using (5.5), (5.6) and (5.11), we have

$$
\begin{aligned}
E\left[B_{\text {in }}\left(S_{\text {in }}, Q_{i}, R_{i}\right)\right] & =\frac{\mu_{\text {in }}}{\mu_{i 0}} E\left[B_{i 0}\left(Q_{i}, R_{i}\right)\right]+E\left[D_{\text {in }}\right]-S_{\text {in }} \\
& +E\left[I_{\text {in }}\left(S_{\text {in }}, Q_{i}, R_{i}\right)\right]
\end{aligned}
$$

where $E\left[D_{i n}\right]=\mu_{i n} T_{i n}$. Then, by substituting (A.1) into the objective function of $S P_{i}(\vec{\theta})$ we obtain 


$$
\begin{aligned}
G\left(Q_{i}, R_{i}, \overrightarrow{S_{i}}\right) & =c_{i} h\left(E\left[I_{i 0}\left(Q_{i}, R_{i}\right)\right]+\sum_{n \in N} E\left[I_{i n}\left(Q_{i}, R_{i}, S_{i n}\right)\right]\right) \\
& +\frac{\mu_{i 0} K_{i}}{Q_{i}}+\theta_{0} E\left[B_{i 0}\left(Q_{i}, R_{i}\right)\right] \\
& +\sum_{n \in N} \theta_{n}\left(\frac{\mu_{i n}}{\mu_{i 0}} E\left[B_{i 0}\left(Q_{i}, R_{i}\right)\right]+E\left[D_{i n}\right]-S_{i n}+E\left[I_{i n}\left(S_{i n}, Q_{i}, R_{i}\right)\right]\right) \\
& =c_{i} h E\left[I_{i 0}\left(Q_{i}, R_{i}\right)\right]+\frac{\lambda_{i 0} K_{i}}{Q_{i}}+\left(\theta_{0}+\sum_{n \in N} \theta_{n} \frac{\mu_{i n}}{\mu_{i 0}}\right) E\left[B_{i 0}\left(Q_{i}, R_{i}\right)\right] \\
& +\sum_{n \in N}\left(c_{i} h+\theta_{n}\right) E\left[I_{i n}\left(Q_{i}, R_{i}, S_{i n}\right)\right]+\sum_{n \in N} \theta_{n}\left(E\left[D_{i n}\right]-S_{i n}\right) .
\end{aligned}
$$

Then, it is a direct consequence of Corollary A.1.3(a) that $G\left(Q_{i}, R_{i}, \overrightarrow{S_{i}^{+}}\right)-G\left(Q_{i}, R_{i}, \overrightarrow{S_{i}}\right) \leq$ $G\left(Q_{i}, R_{i}^{+}, \overrightarrow{S_{i}^{+}}\right)-G\left(Q_{i}, R_{i}^{+}, \overrightarrow{S_{i}}\right)$.

Proof of part (b): After following the steps of the proof of part (a), the rest of the proof follows from Corollary A.1.3(b).

Corollary A.1.5 For a given value of $Q_{i}$, the optimal $R_{i}$ as $S_{\text {in }} \rightarrow \infty$ for each $n \in N$, i.e., $R_{i}^{*}\left(Q_{i}, \lim _{\vec{S}_{i} \rightarrow \vec{\infty}} \vec{S}_{i}\right)$ is a lower bound on the optimal $R_{i}$; the optimal $R_{i}$ for $S_{i n}=0$ for each $n \in N$, i.e., $R_{i}^{*}\left(Q_{i}, \vec{S}_{i}=\overrightarrow{0}\right)$ is an upper bound on the optimal $R_{i}$.

Proposition A.1.6 For any $R_{i}^{+}>R_{i}, Q_{i}^{*}\left(R_{i}, \lim _{\vec{S}_{i \rightarrow \infty}} \vec{S}_{i}\right) \geq Q_{i}^{*}\left(R_{i}^{+}, \lim _{\vec{S}_{i} \rightarrow \vec{\infty}} \vec{S}_{i}\right)$.

Proof. By using (A.1) into the objective function of $S P_{i}(\vec{\theta})$ we establish

$$
\begin{aligned}
G\left(Q_{i}, R_{i}, \overrightarrow{S_{i}}\right) & =c_{i} h E\left[I_{i 0}\left(Q_{i}, R_{i}\right)\right]+\frac{\mu_{i 0} K_{i}}{Q_{i}}+\left(\theta_{0}-c_{i} h \sum_{n \in N} \frac{\mu_{i n}}{\mu_{i 0}}\right) E\left[B_{i 0}\left(Q_{i}, R_{i}\right)\right] \\
& +c_{i} h \sum_{n \in N}\left(S_{i n}-E\left[D_{i n}\right]\right)+\sum_{n \in N}\left(c_{i} h+\theta_{n}\right) E\left[B_{i n}\left(Q_{i}, R_{i}, S_{i n}\right)\right] .
\end{aligned}
$$

Then, for any given value of $S_{i n}, c_{i} h \sum_{n \in N}\left(S_{i n}-E\left[D_{i n}\right]\right)$ is a constant, so it can be excluded from the optimization of (A.3) over $Q_{i}$. As $S_{i n} \rightarrow \infty$ for each $n \in N$, $E\left[B_{i n}\left(Q_{i}, R_{i}, S_{i n}\right)\right]=0$. In this situation, optimizing (A.3) reduces to minimizing $\bar{G}\left(Q_{i}, R_{i}\right)=c_{i} h E\left[I_{i 0}\left(Q_{i}, R_{i}\right)\right]+\frac{\mu_{i 0} K_{i}}{Q_{i}}+\left(\theta_{0}-c_{i} h \sum_{n \in N} \frac{\mu_{i n}}{\mu_{i 0}}\right) E\left[B_{i 0}\left(Q_{i}, R_{i}\right)\right]$. This function 
corresponds to the cost function for single echelon $(Q, R)$ policy, for which we have $Q_{i}^{*}\left(R_{i}\right) \geq Q_{i}^{*}\left(R_{i}^{+}\right)$.

Proposition A.1.7 $Q_{i}^{*}\left(\lim _{R_{i} \rightarrow \infty} R_{i}, \lim _{\vec{S}_{i} \rightarrow \infty} \vec{S}_{i}\right)$ is a lower bound on the optimal $Q_{i}$.

Proof. Proof follows from Theorem A.1.4(b) and Proposition A.1.6. The rest of the proof is the same as that of Proposition 3.2.7 in Section 3.2.2.

Proposition A.1.8 $Q_{i}^{U B}=\sqrt{\left(2 K_{i} \mu_{i 0}+\left(c_{i} h+p_{i}\right) \operatorname{Var}\left(D_{i 0}\right)\right) / H_{i}}$, where $H_{i}=\frac{c_{i} h p_{i}}{c_{i} h+p_{i}}$, and $p_{i}=\theta_{0}+\sum_{n \in N} \theta_{n} \frac{\mu_{i n}}{\mu_{i 0}}$, is an upper bound on the optimal $Q_{i}$.

Proof. The proof will be complete if we show that $G\left(Q_{i}, R_{i}, \vec{S}_{i}\right)>G\left(Q_{i}^{-}, R_{i}, \overrightarrow{S_{i}}\right)$ for any $Q_{i}>Q_{i}^{U B}, \vec{S}_{i}$ and $R_{i}$, where $Q_{i}^{-}<Q_{i}$. Let $C\left(Q_{i}, R_{i}\right)=c_{i} h E\left[I_{i 0}\left(Q_{i}, R_{i}\right)\right]+\frac{\lambda_{i 0} K_{i}}{Q_{i}}+$ $\left(\theta_{0}+\sum_{n \in N} \theta_{n} \frac{\lambda_{i n}}{\lambda_{i 0}}\right) E\left[B_{i 0}\left(Q_{i}, R_{i}\right)\right]$. We note that $C\left(Q_{i}, R_{i}\right)$ corresponds to the cost function in Gallego (1998). Based on this study, $Q_{i}^{U B}$ is an upper bound on the optimal $Q_{i}$ for $C\left(Q_{i}, R_{i}\right)$. Furthermore, since $C\left(Q_{i}, R_{i}\right)$ is unimodal with respect to $Q_{i}$ (Federgruen and Zipkin 1992), for any $Q_{i}>Q_{i}^{U B}$, we have $C\left(Q_{i}, R_{i}\right)>C\left(Q_{i}^{-}, R_{i}\right)$. Finally, using this result, $E\left[I_{i n}\left(Q_{i}, R_{i}, S_{i n}\right)\right] \geq E\left[I_{i n}\left(Q_{i}^{-}, R_{i}, S_{i n}\right)\right]$, and that $\sum_{n \in N} \theta_{n}\left(E\left[D_{i n}\right]-S_{i n}\right)$ is constant with respect to $Q_{i}$ in (A.2), we establish $G\left(Q_{i}, R_{i}, \overrightarrow{S_{i}}\right)>G\left(Q_{i}^{-}, R_{i}, \overrightarrow{S_{i}}\right)$.

\section{A.1.1 Finding Optimal Solution for Subproblems for Given Values of Reorder Level and Order Quantity}

For a given part $i \in I$, and given values of $Q_{i}$ and $R_{i}, S P_{i}(\vec{\theta})$ reduces to $|N|$ independent subproblems, each of which is denoted by $S P_{i n}\left(\theta_{n}, Q_{i}, R_{i}\right)$.

$$
\begin{array}{ll}
\text { Min } & c_{i} h E\left[I_{i n}\left(Q_{i}, R_{i}, S_{i n}\right)\right]+\theta_{n} E\left[B_{i n}\left(Q_{i}, R_{i}, S_{i n}\right)\right] \\
\text { s.t. } & S_{\text {in }} \geq 0 \text {, and } \in Z .
\end{array}
$$

By substituting (5.11), the objective function of $S P_{i n}\left(\theta_{n}, Q_{i}, R_{i}\right)$ can be restated as

$$
G\left(S_{i n}\right)=\left(c_{i} h+\theta_{n}\right) E\left[I_{i n}\left(Q_{i}, R_{i}, S_{i n}\right)\right]+\theta_{n} E\left[X_{i n}\left(Q_{i}, R_{i}\right)\right]-\theta_{n} S_{\text {in }} .
$$


Proposition A.1.9 $G\left(S_{\text {in }}\right)$ is unimodal.

Proposition A.1.10 The optimal solution of $S P_{i n}\left(\theta_{n}, Q_{i}, R_{i}\right)$ is

$$
\operatorname{Min}_{S_{i n} \in\{0,1,2, \ldots\}}\left\{S_{i n}: \sum_{x=0}^{S_{i n}} P\left(X_{i n}\left(Q_{i}, R_{i}\right)=x\right) \geq \frac{\theta_{n}}{c_{i} h+\theta_{n}}\right\} .
$$

Proof. Proofs of Proposition A.1.9 and A.1.10 are the same as that of Proposition 3.2.9 and 3.2.10 in Section 3.2.2. 


\section{APPENDIX B}

THE RESULTS OF THE EXPERIMENTERS FOR TESTBED 1 FOR THE SEQUENTIAL HEURISTICS 


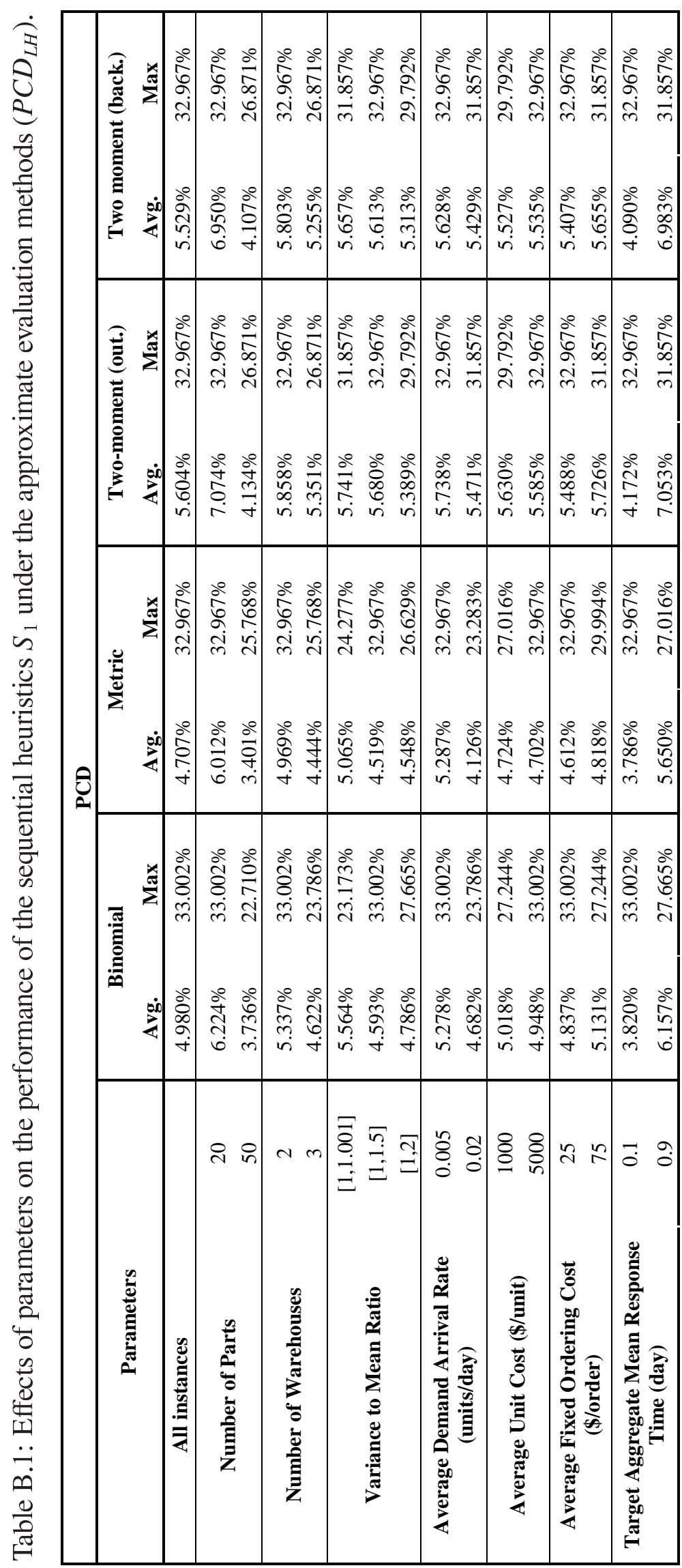




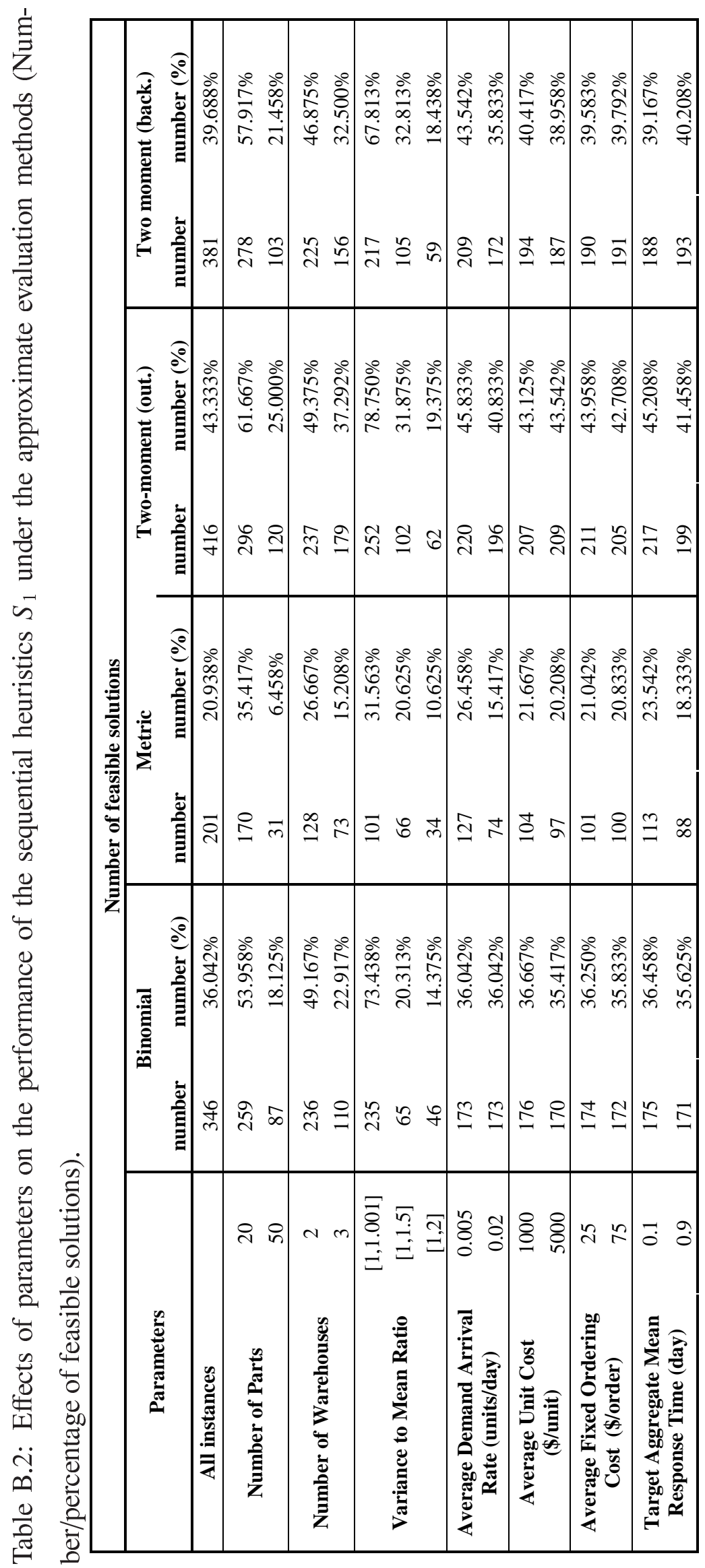




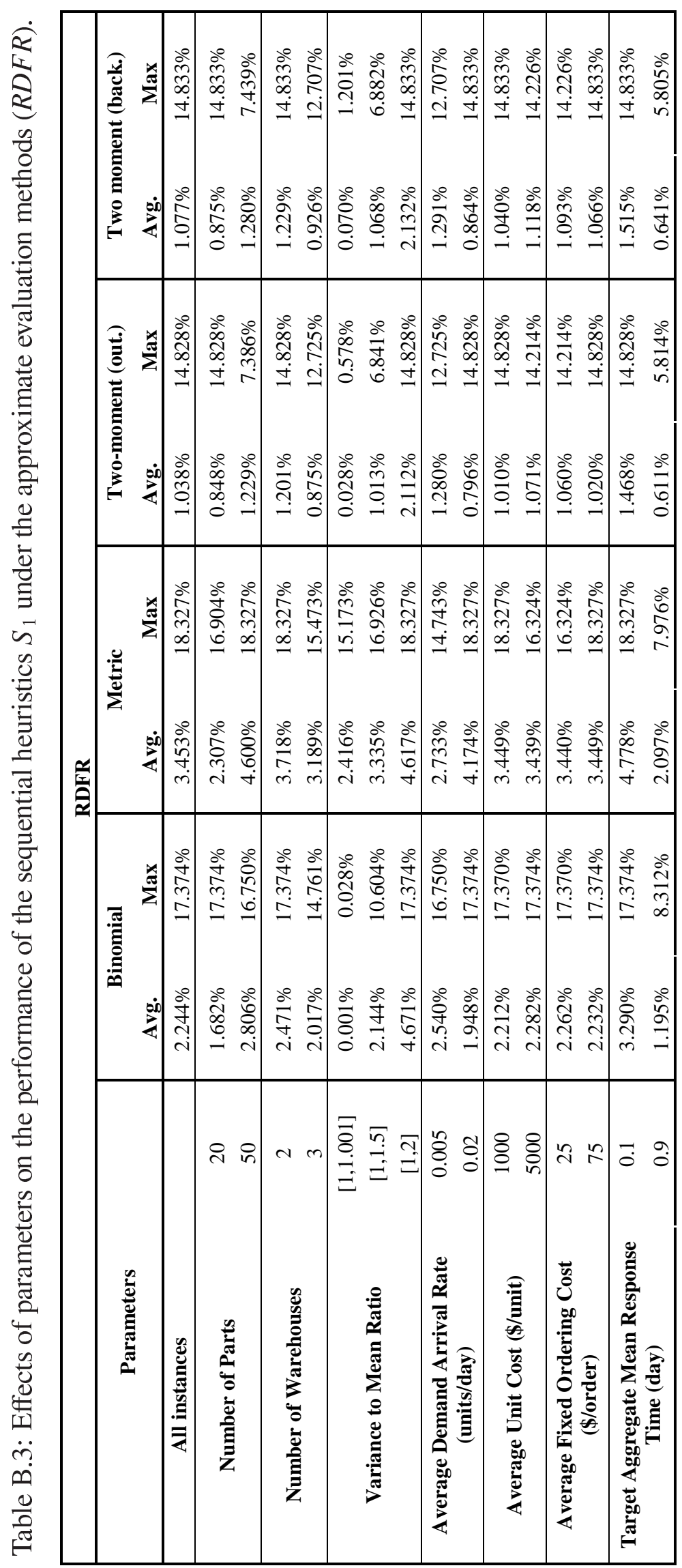




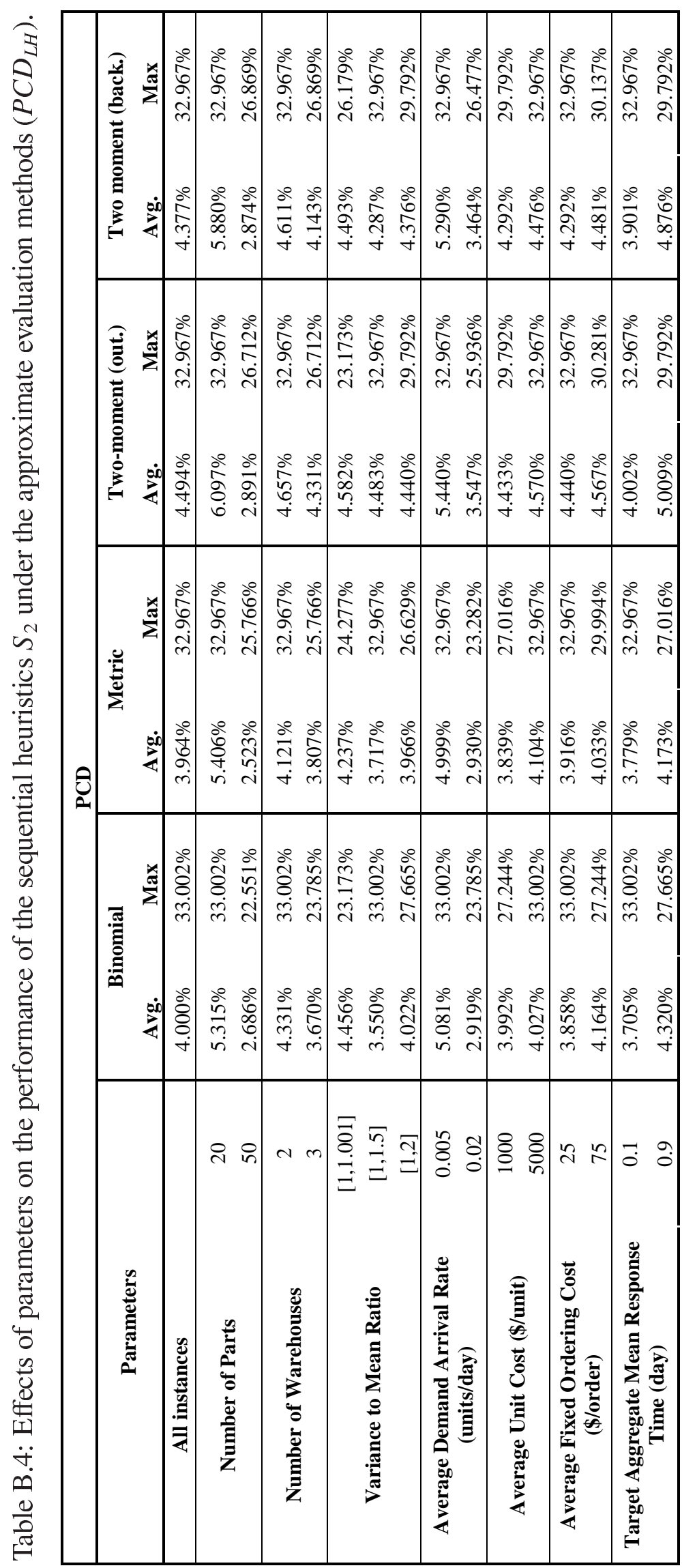




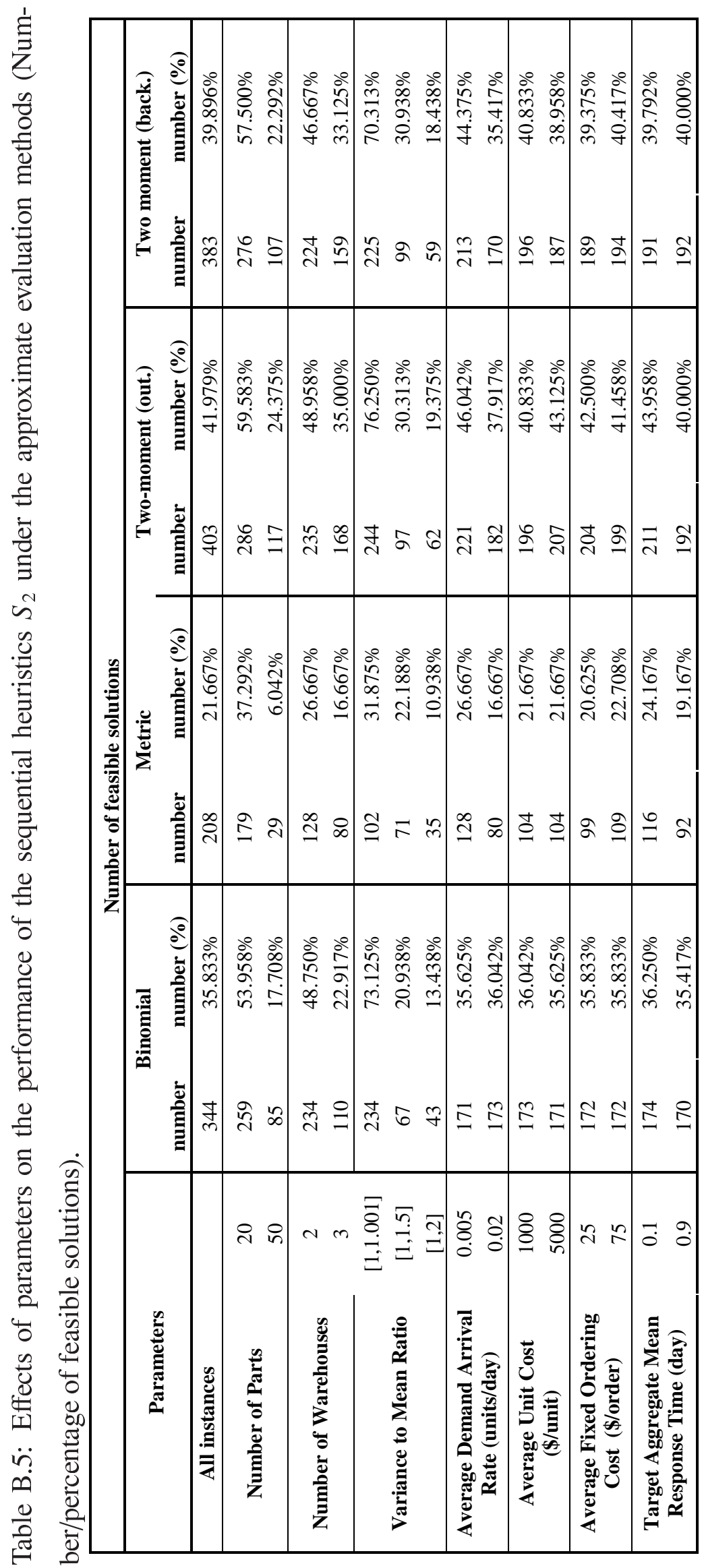




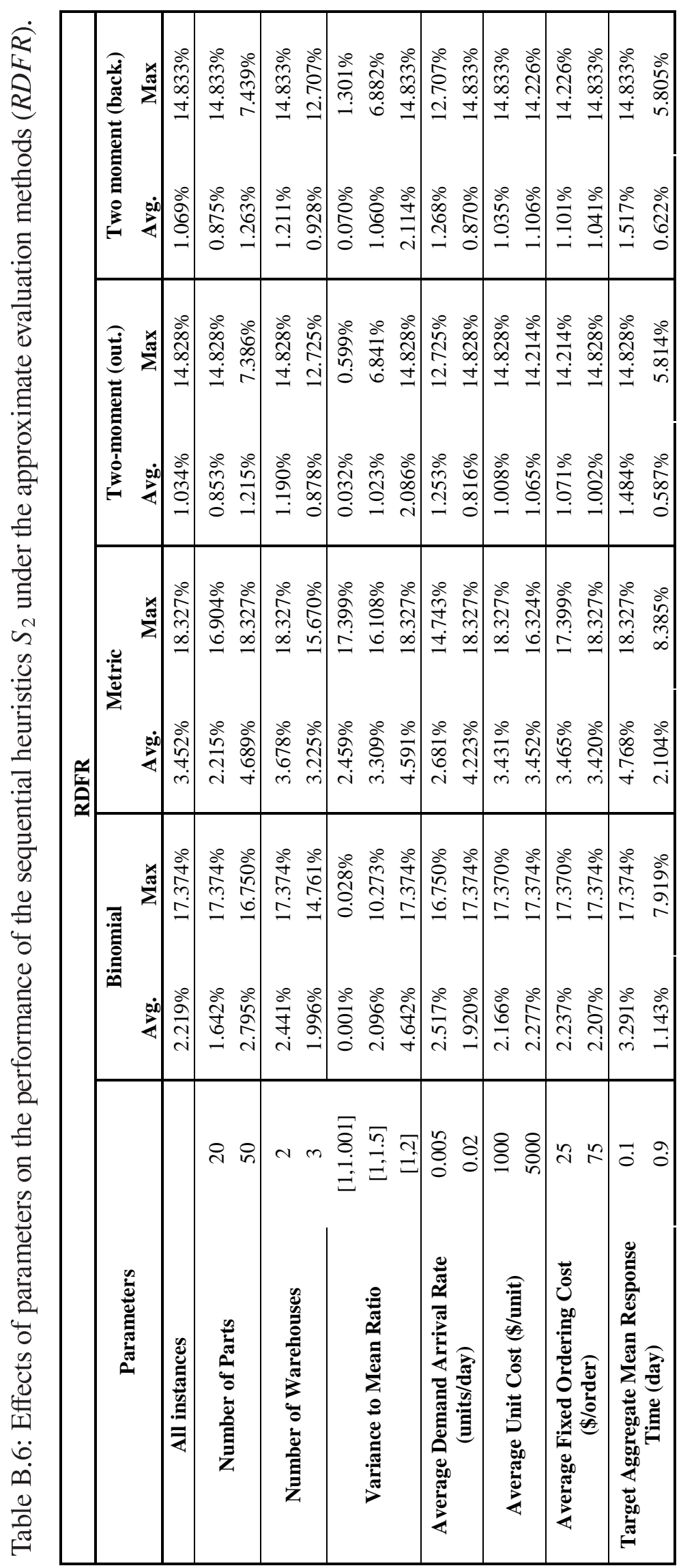




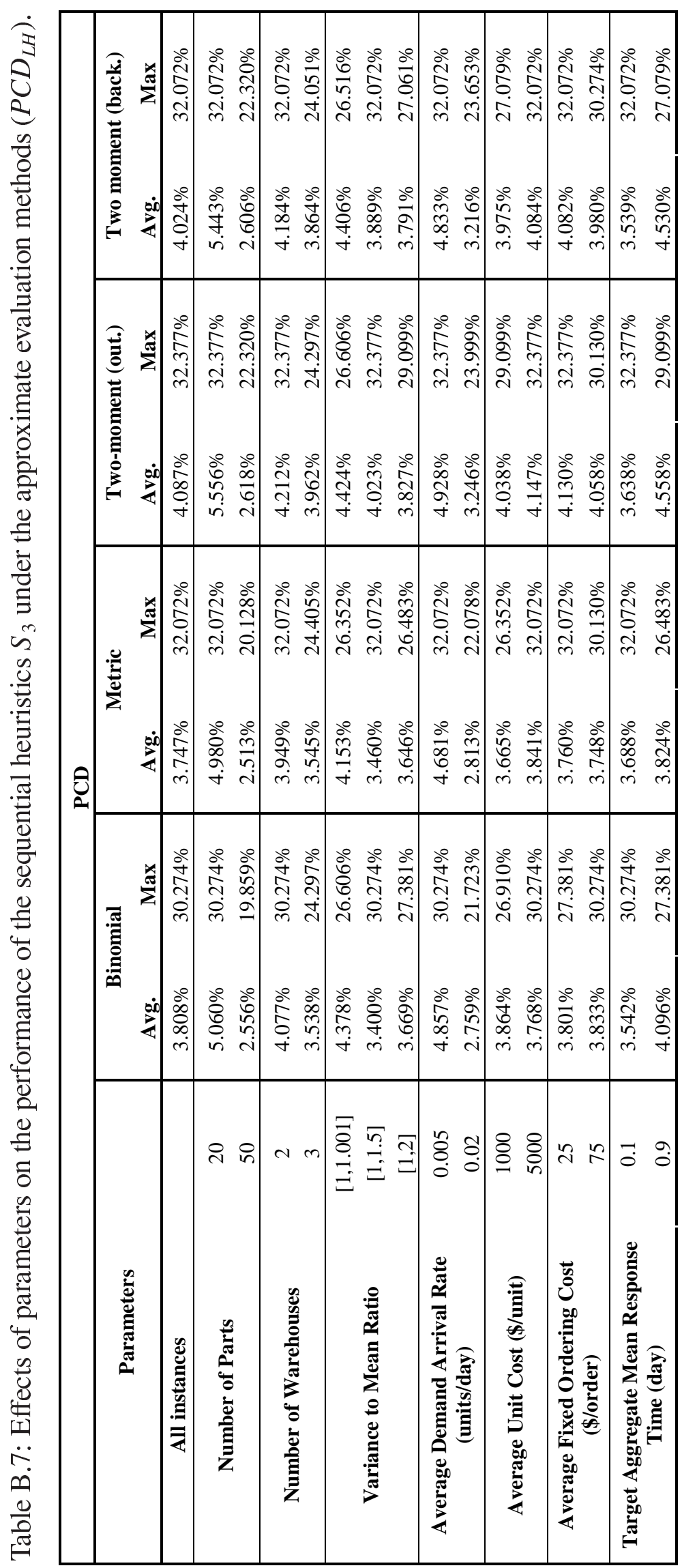




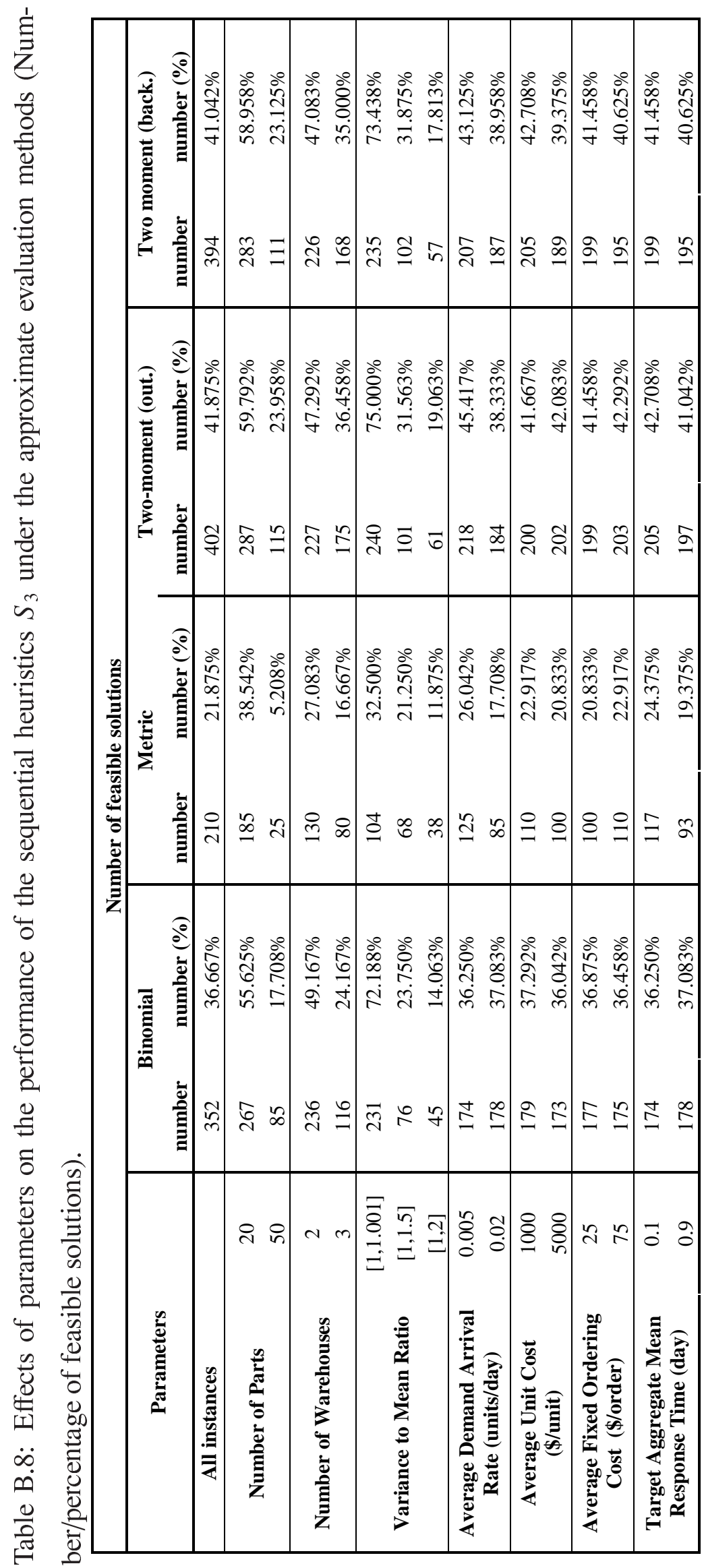




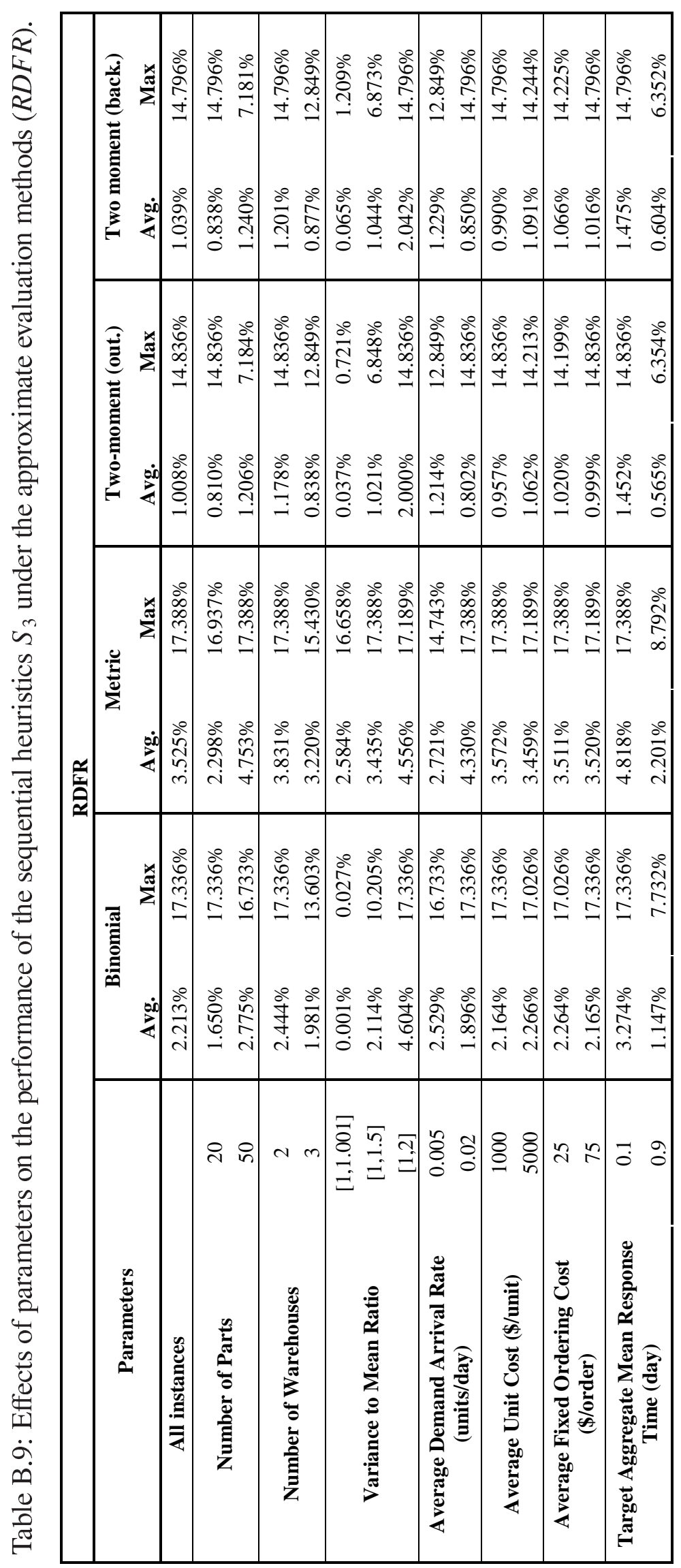




\section{APPENDIX C}

\section{NOTATIONS USED IN THE DISSERTATION}

Notations used in the dissertation are given below.

\section{PARAMETERS and VARIABLES}

$I:$ Set of parts

$i:$ Part index, $i \in I$

$N$ : Set of Local warehouses

$n$ : Warehouse index $n \in N \cup\{0\}$

$c_{i}$ : Unit variable cost of part $i \in I$

$h$ : Inventory carrying charge

$K_{i}$ : Fixed ordering cost of part $i \in I$ at the central warehouse

$\lambda_{\text {in }}$ : Customer arrival rate for part $i \in I$ at local warehouse $n \in N$

$\lambda_{i 0}^{e}$ : External customer arrival rate for part $i \in I$ at the central warehouse

$\lambda_{i 0}$ : Customer arrival rate (sum of internal and external) for part $i \in I$ at the central warehouse

$\mu_{\text {in }}$ : Demand rate for part $i \in I$ at local warehouse $n \in N$

$\mu_{i 0}^{e}$ : External demand rate for part $i \in I$ at the central warehouse

$\mu_{i 0}$ : Demand rate (sum of internal and external) for part $i \in I$ at the central warehouse

$\sigma_{i n}^{2}$ : Total demand variance for part $i \in I$ at local warehouse $n \in N \cup\{0\}$

$M_{n}^{e}$ : Total external demand rate at the central warehouse

$M_{n}$ : Total demand rate for warehouse $n \in N \cup\{0\}$

$T_{i 0}$ : Lead time for part $i \in I$ at the central warehouse from the outside supplier

$T_{i n}$ : Transportation lead time $T_{i n}$ from the central warehouse to local warehouse $n \in N$ for part $i \in I$ 
$Y_{\text {in }}$ : Number of customer arrivals during lead time $T_{\text {in }}$ at warehouse $n \in N \cup\{0\}$ for part $i \in I$

$V_{\text {in }}$ : Demand size for part $i \in I$ at warehouse $n \in N \cup\{0\}$

$D_{\text {in }}$ : Total demand during lead time $T_{\text {in }}$ at warehouse $n \in N \cup\{0\}$ for part $i \in I$

$Y_{i 0}^{e}$ : Number of external customer arrivals during lead time $T_{i 0}$ at the central warehouse for part $i \in I$

$V_{i 0}^{e}$ : External demand size for part $i \in I$ at the central warehouse

$D_{i 0}^{e}$ : Total external demand during lead time $T_{i 0}$ at the central warehouse for part $i \in I$ $v_{i n}^{k}$ : Size of $k^{\text {th }}$ demand occurred during the lead time $T_{\text {in }}$ at warehouse $n \in N \cup\{0\}$ for part $i \in I$

$W_{n}^{\max }$ : Target aggregate mean response time at warehouse $n \in N \cup\{0\}$

$R_{i}:$ Reorder level for part $i \in I$ at the central warehouse

$Q_{i}$ : Order quantity for part $i \in I$ at the central warehouse

$S_{\text {in }}$ : Base-stock level for part $i \in I$ at local warehouse $n \in N$

$\vec{S}_{i}:\left[S_{i 1}, S_{i 2}, \ldots, S_{i|N|}\right]=$ Vector of base-stock levels for part $i \in I$

$\vec{S}:\left[\vec{S}_{1}, \vec{S}_{2}, \ldots, \vec{S}_{|I|}\right]=$ Vector of base-stock levels

$\vec{Q}:\left[Q_{1}, Q_{2}, \ldots, Q_{|I|}\right]=$ Vector of order quantities

$\vec{R}:\left[R_{1}, R_{2}, \ldots, R_{|I|}\right]=$ Vector of reorder levels

$I_{i n}\left(Q_{i}, R_{i}, S_{i n}\right)$ : On-hand inventory level for part $i \in I$ at warehouse $n \in N$ in the steady state

$I_{i 0}\left(Q_{i}, R_{i}\right)$ : On-hand inventory level for part $i \in I$ at the central warehouse in the steady state

$X_{i n}\left(Q_{i}, R_{i}\right)$ : Number of outstanding orders for part $i \in I$ at warehouse $n \in N$ in the steady state

$B_{\text {in }}\left(Q_{i}, R_{i}, S_{\text {in }}\right)$ : Backorder level for part $i \in I$ at warehouse $n \in N$ in the steady state $B_{i 0}\left(Q_{i}, R_{i}\right)$ : Backorder level for part $i \in I$ at the central warehouse in the steady state $B_{i 0}^{(n)}\left(Q_{i}, R_{i}\right)$ : Backorder level of local warehouse $n \in N$ for part $i \in I$ at the central warehouse in the steady state

$W_{i n}\left(Q_{i}, R_{i}, S_{i n}\right):$ Response time for part $i \in I$ at warehouse $n \in N$ in the steady state $W_{i 0}\left(Q_{i}, R_{i}\right):$ Response time for part $i \in I$ at the central warehouse in the steady state $W_{i 0}^{e}\left(Q_{i}, R_{i}\right)$ : Response time for part $i \in I$ at the central warehouse (based on external customers)

$W_{n}(\vec{Q}, \vec{R}, \vec{S})$ : Aggregate mean response time at warehouse $n \in N$ in the steady state 
$W_{0}(\vec{Q}, \vec{R})$ : Aggregate mean response time at the central warehouse in the steady state $W_{0}^{e}(\vec{Q}, \vec{R})$ : Aggregate mean response time at the central warehouse (based on external customers)

$L:$ Set of columns in master problem $M P$

$l$ : Column index, $l \in L$

$x_{i l}$ : variable indicating whether column $l \in L$ is selected for part $i \in I$ or not in master problem $M P$

$C_{i l}$ : expected total inventory holding and fixed ordering costs associated with column $l \in L$ for part $i \in I$ in master problem $M P$

$A_{i l n}: \frac{E\left[B_{i n}\right]}{\Lambda_{n}}$, technological coefficient associated with column $l \in L$ for part $i \in I$ for warehouse $n \in N \cup\{0\}$ in master problem $M P$

$\alpha_{n}$ : Lagrangian multiplier for the constraint (3.12) associated with warehouse $n \in$ $N \cup\{0\}$ in master problem $M P$

$\beta_{i}$ : Lagrangian multiplier for the constraint (3.13) associated with part $i \in I$ in master problem $M P$

$\theta_{n}: \frac{-\alpha_{n}}{\Lambda_{n}}$, penalty cost implied by relaxation of the aggregate mean response time constraint for warehouse $n \in N \cup\{0\}$

$\vec{\theta}:\left[\theta_{1}, \theta_{2}, \ldots, \theta_{|N|}\right]$

$Z(\vec{\theta})$ : Optimal objective function value for problem $C G$

$Z_{i}(\vec{\theta})$ : Optimal objective function value for problem $S P_{i}(\vec{\theta})$

$Q_{i}^{L B}$ : Lower bound for the optimal values for $Q_{i}$

$Q_{i}^{U B}$ : Upper bound for the optimal values for $Q_{i}$

$R_{i}^{L B}$ : Lower bound for the optimal values for $R_{i}$

$R_{i}^{U B}$ : Upper bound for the optimal values for $R_{i}$

$p_{i}: \theta_{0}+\sum_{n \in N} \theta_{n} \frac{\lambda_{i n}}{\lambda_{i 0}}$, shortage cost defined per unit short of part $i \in I$

$H_{i}: \frac{c_{i} h p_{i}}{c_{i} h+p_{i}}$

$\gamma_{i}$ : Probability of no stockouts for part $i \in I$

$E O Q: \sqrt{\frac{2 \lambda_{i 0} K_{i}}{c_{i} h}}$, economic order quantity, batch size heuristic considered for sequential heuristic $S_{2}$

$E O Q^{B}: \sqrt{\frac{2 \lambda_{i 0} K_{i}\left(c_{i} h+p_{i}\right)}{\left(c_{i} h\right) p_{i}}}$, EOQ with planned backorders, batch size heuristic considered for sequential heuristic $S_{2}$

$Q^{L U}: \min \left(\sqrt{2} Q_{i}^{L B}, \sqrt{Q_{i}^{L B} \cdot Q_{i}^{U B}}\right)$, batch size heuristic considered for sequential heuristic $S_{3}$ 
$z^{*}, z^{P}$ : Optimal objective function value of problem $P$

$z$ : Objective function value of any solution to be tested

$z^{M P}$ : Optimal objective function value of problem $M P$

$z^{L P M P}:$ Optimal objective function value of problem LPMP

$z_{L D}$ : Objective function value of the solution obtained by the Lagrangian dual solution (Lagrangian dual bound) for problem $P$

$z^{\prime}$ : Objective function value of the Lagrangian dual solution obtained by any approximate evaluation method to be tested

$z^{L H}$ : Objective function value of the solution obtained by the Lagrangian heuristic when the exact method is used

$z_{a p p}:$ Objective function value of the solution of the Lagrangian heuristic that is obtained by using the two-moment approximation for outstanding orders

$z_{i}^{E O Q\left(\theta_{0}\right)}: \sqrt{\frac{2 K_{i} \lambda_{i 0} c_{i} h \theta_{0}}{c_{i} h+\theta_{0}}}$, optimal objective function value of the EOQ model with unit backorder cost of $\theta_{0}$

$\rho_{d}$ : demand skewness parameter

$\lambda$ : average demand rate of all parts

$\rho_{c}:$ cost skewness parameter

$c$ : average unit variable cost of parts

$v_{i}$ : part-specific average demand rate for part $i \in I$

$P\left(\mathcal{V}_{i n}^{k}=x\right): k$-fold convolution of $P\left(V_{\text {in }}=v\right)$, probability that $k$ customers yields a total demand of $x$ for part $i \in I$ at warehouse $n \in N$

$P G A P: \frac{\left|z^{L D}-z^{*}\right|}{z^{*}}$, percentage dual gap with the optimal expected total cost

$G A P:\left|z^{L D}-z^{*}\right|$, absolute dual gap with the optimal expected total cost

$P C D: \frac{\left|z-z^{L D}\right|}{z^{L D}}$, percentage cost difference between the expected total cost of the solution to be tested and the Lagrangian dual bound

$A C D:\left|z-z^{L D}\right|$, absolute cost difference between the expected total cost of the solution to be tested and the Lagrangian dual bound

$R D F R: \sum_{n \in N \cup\{0\}}\left\{\left(W_{n}(X)-W_{n}^{\max }\right)^{+}\right\} / \sum_{n \in N \cup\{0\}} W_{n}^{\max }$, relative distance to the feasible region

$P G A P^{\prime}: \frac{\left|z^{\prime}-z^{L D}\right|}{z^{L D}}$, percentage gap between the expected total cost of the Lagrangian dual solution obtained by the approximate evaluation method to be tested and the Lagrangian dual bound

$P C D_{L H}: \frac{\left|z-z^{L H}\right|}{z^{L H}}$, percentage cost difference between the expected total cost of the solu- 
tion to be tested and the expected total cost of the solution of the Lagrangian heuristic (that is obtained by using the exact evaluation)

$P C D_{L D}: \frac{\left|z-z^{L D}\right|}{z^{L D}}$, percentage cost difference between the expected total cost of the solution to be tested and the Lagrangian dual bound

PCD $D_{\text {LHapp }}: \frac{\left|z-z_{a p p}\right|}{z_{a p p}}$, percentage cost difference between the expected total cost of the solution to be tested and the expected total cost of the solution of the Lagrangian heuristic that is obtained by using the two-moment approximation for outstanding orders

\section{ABBREVIATIONS}

$P$ : Original problem considered in the dissertation

$M P$ : Master problem for problem $P$

$L P M P$ : $L P$-relaxation of master problem $M P$

$R M P$ : Restricted master problem

$C G$ : Column generation or pricing problem

$A P$ : Alternative formulation of problem $M P$

$L P A P$ : $L P$-relaxation of problem $A P$

$S P_{i}(\vec{\theta})$ : Subproblem for part $i \in I$ for a given $\vec{\theta}$

$P_{c}$ : Original problem for the compound Poisson demand model

$S_{1}:$ Sequential heuristic based on the EOQ

$S_{2}$ : Sequential heuristic based on the EOQ with planned backorders

$S_{3}$ : Sequential heuristic based on $Q^{L U}$

$\mathbf{O}(g)$ : Asymptotically bounded above by function $g$

$L W H$ : Local warehouse

NumPart: Number of parts

$D C G$ : Decomposition and column generation

FCFS : First come, first served 


\section{VITA}

\section{PERSONAL INFORMATION}

Surname, Name: Topan, Engin

Nationality: Turkish (TC)

Date and Place of Birth: 27 April 1979, Muğla

Marital Status: Married with İpek Seyran Topan

Phone: +90 3122844500 / 4098

email: etopan@cankaya.edu.tr

\section{EDUCATION}

$\begin{array}{lll}\text { Degree } & \text { Institution } & \text { Year of Graduation } \\ \text { MS } & \text { METU Industrial Engineering } & 2005 \\ \text { BS } & \text { METU Industrial Engineering } & 2002 \\ \text { High School } & \text { İzmir Vecdi Gönül } & 1997 \\ \text { High School } & \text { İzmir Science } & 1994-1996\end{array}$

WORK EXPERIENCE

$\begin{array}{lll}\text { Year } & \text { Place } & \text { Enrollment } \\ \text { 2009-Present } & \text { Çankaya University } & \text { Instructor } \\ \text { 2002-2009 } & \text { Çankaya University } & \text { Teaching Assistant } \\ \text { High School } & \text { Pınar Dairy } & \text { Intern Engineering Student } \\ \text { High School } & \text { Vestel Electronics } & \text { Intern Engineering Student }\end{array}$

FOREIGN LANGUAGES

Advanced English 


\section{PUBLICATIONS}

1. E. Topan and Z.M. Avşar. An Approximation for Kanban Controlled Assembly Systems. Annals of Operations Research, DOI 10.1007/s10479-009-0560-1, 2009.

2. E. Topan, Z.P. Bayındır, and T. Tan. An Exact Solution Procedure for MultiItem Two Echelon Spare Parts Inventory Control Problem with Batch Ordering in the Central Warehouse. Operations Research Letters, 38, 454-461, 2010.

\section{HOBBIES}

Reading (more on history), Movies, Computer games 